\title{
LINEAR DYNAMICAL SYSTEMS ON HILBERT SPACES: TYPICAL PROPERTIES AND EXPLICIT EXAMPLES
}

\author{
by
}

\author{
S. Grivaux, É. Matheron \& Q. Menet
}

\begin{abstract}
We solve a number of questions pertaining to the dynamics of linear operators on Hilbert spaces, sometimes by using Baire category arguments and sometimes by constructing explicit examples. In particular, we prove the following results.

(i) A typical hypercyclic operator is not topologically mixing, has no eigenvalues and admits no non-trivial invariant measure, but is densely distributionally chaotic.

(ii) A typical upper-triangular operator is ergodic in the Gaussian sense, whereas a typical operator of the form "diagonal plus backward unilateral weighted shift" is ergodic but has only countably many unimodular eigenvalues; in particular, it is ergodic but not ergodic in the Gaussian sense.

(iii) There exist Hilbert space operators which are chaotic and $\mathcal{U}$-frequently hypercyclic but not frequently hypercyclic, Hilbert space operators which are chaotic and frequently hypercyclic but not ergodic, and Hilbert space operators which are chaotic and topologically mixing but not $\mathcal{U}$-frequently hypercyclic.

We complement our results by investigating the descriptive complexity of some natural classes of operators defined by dynamical properties.
\end{abstract}

\section{Contents}

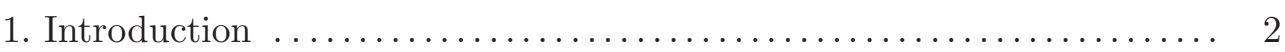

2. Typical properties of hypercyclic operators $\ldots \ldots \ldots \ldots \ldots \ldots \ldots, 11$

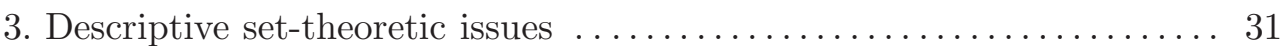

4. Ergodicity for upper-triangular operators $\ldots \ldots \ldots \ldots \ldots \ldots \ldots \ldots .45$

5. Periodic points at the service of hypercyclicity $\ldots \ldots \ldots \ldots \ldots \ldots . . \ldots 5$

6. Special examples of hypercyclic operators $\ldots \ldots \ldots \ldots \ldots \ldots \ldots \ldots . . \ldots 1$

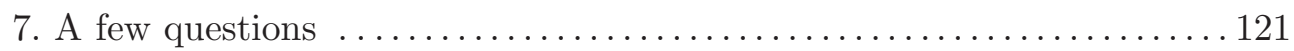

References .................................................

2000 Mathematics Subject Classification. - 47A16, 37A05, 54E52, 54H05.

Key words and phrases. — Linear dynamical systems; Hilbert spaces; Baire category; frequent and $\mathcal{U}$-frequent hypercyclicity; ergodicity; chaos; topological mixing.

The work of the first-named author is supported in part by EU IRSES grant AOS (PIRSES-GA-2012318910). 


\section{Introduction}

1.1. General overview. - This paper is a contribution to the study of linear dynamical systems on Hilbert spaces. In other words, we are interested in the behavior of orbits of bounded linear operators defined on a Hilbert space. The symbol $\mathcal{H}$ will always designate a complex separable infinite-dimensional Hilbert space, and we denote by $\mathfrak{B}(\mathcal{H})$ the algebra of all bounded linear operators on $\mathcal{H}$.

We refer to the books [7] or [31 for background on linear dynamics, and to the papers 25] or 28 for a glimpse at the richness of the class of linear dynamical systems and its potential usefulness in general ergodic theory. A quick review of a number of definitions will be given in the next subsection. Let us just recall here that an operator $T \in \mathfrak{B}(\mathcal{H})$ is said to be hypercyclic if it admits at least one dense orbit (and hence a dense $G_{\delta}$ set of such orbits). The class of all hypercyclic operators on $\mathcal{H}$ will be denoted by $\operatorname{HC}(\mathcal{H})$. Recall also that an operator $T \in \mathfrak{B}(\mathcal{H})$ is said to be chaotic if it is hypercyclic and admits a dense set of periodic vectors, and frequently hypercyclic (resp. $\mathcal{U}$-frequently hypercyclic) if there exists at least one vector $x \in \mathcal{H}$ whose orbit visits frequently every non-empty open set $V \subseteq \mathcal{H}$, in the sense that the set of integers $i \in \mathbb{N}$ such that $T^{i} x$ belongs to $V$ has positive lower (resp. upper) density.

Obviously, the dynamical properties of linear operators can be studied (and have been studied extensively) in a very general setting - arbitrary Banach spaces, or even arbitrary topological vector spaces. However, there are good reasons for focusing on Hilbert spaces. The first that may come to mind is perhaps that "this is the natural setting for doing operator theory" - which is of course a highly questionable statement. Less subjectively, the richness of the Hilbert space structure allows for the construction of many interesting examples. Also, in some parts of linear dynamics, especially all that concerns ergodictheoretic properties of linear operators, the general picture is neater on Hilbert spaces than on arbitrary Banach spaces. Finally, some natural questions in the area have been solved recently for operators on general Banach space but not in the Hilbertian case. This should not seem paradoxical, if one compares for example with the current state of affairs regarding the famous Invariant Subspace Problem.

The questions we are considering in this paper are quite basic. In very general terms, they are of the following type: given two interesting dynamical properties, do there exist linear operators on $\mathcal{H}$ satisfying one of them but not the other?

Perhaps the most famous question of this type is Herrero's " $T \oplus T$ problem", stated in [35] and asking whether there exist hypercyclic operators which are not topologically weakly mixing. As should be expected since this is trivially true for dynamical systems on compact spaces, the answer is "Yes": this was shown by De La Rosa and Read in [18, and then in [6] for operators on $\ell_{p}$ spaces, in particular for Hilbert space operators. However, the proof is surprisingly non-trivial. To give just one more example, the third named author was recently able to solve another well-known problem in the area by constructing in [39] operators on $\ell_{p}$ spaces which are chaotic but not frequently hypercyclic - actually, not even $\mathcal{U}$-frequently hypercyclic. In the present paper, we will be especially interested in the following questions:

- are there operators which are frequently hypercyclic but not ergodic, i.e. do not admit an ergodic measure with full support?

- are there operators which are $\mathcal{U}$-frequently hypercyclic but not frequently hypercyclic? 
- are there operators which are ergodic but do not admit a Gaussian ergodic measure with full support?

Note that the answer to the first two questions is known to be "Yes" for operators on the Banach space $c_{0}$ (see [9] and 29, as well as [14 for further developments); but the Hilbertian case had not been settled. As for the third question, we are not aware of any previous answer, on any Banach space.

One can attack questions of this type from two complementary points of view (of course, there are other strategies as well).

- "Collective" point of view: among the properties one is interested in, one may try to determine which ones are generic and which ones are not, in a Baire category sense. Once this is done, one may be able to distinguish two properties because one of them is generic and the other one is not.

- "Individual" point of view: when it is unclear how a Baire category approach could work, one may still try to construct explicit examples of operators satisfying (or not) such or such a property.

Note that it is quite natural to present the two viewpoints in this order, because the indirect, Baire category approach is in some sense simpler. Indeed, it is usually not too difficult to guess when it should work, and in this case the technical details are likely to be rather soft. On the other hand, one may reasonably expect to face technical difficulties when attempting a direct construction (and perhaps quite serious ones if Baire category is unefficient).

To give meaning to the "collective" viewpoint, one has to fix an appropriate topological setting. We will in fact consider two natural topologies on $\mathfrak{B}(\mathcal{H})$ : the Strong Operator Topology (denoted by SOT), and the Strong* Operator Topology (denoted by SOT*). Restricted to any closed ball $\mathfrak{B}_{M}(\mathcal{H}):=\{T \in \mathfrak{B}(\mathcal{H}) ;\|T\| \leq M\}$, these topologies are Polish (separable and completely metrizable). Moreover, $\mathrm{HC}(\mathcal{H})$ is easily seen to be $\mathrm{SOT}-G_{\delta}$ in $\mathfrak{B}(\mathcal{H})$, so that for any $M>1$, the family $\operatorname{HC}_{M}(\mathcal{H}):=\{T \in \mathrm{HC}(\mathcal{H}) ;\|T\| \leq M\}$ is itself a Polish space in its own right with respect to both SOT and SOT*. This opens the way to Baire category arguments in $\mathfrak{B}_{M}(\mathcal{H})$ or $\mathrm{HC}_{M}(\mathcal{H})$. Of course, there is nothing new in this observation: Baire category methods have already proved as useful in operator theory as anywhere else; see in particular [21].

Recall that a subset of a Polish space $X$ is said to be meager in $X$ if it can be covered by countably many closed sets with empty interior, and comeager if its complement is meager (equivalently, if it contains a dense $G_{\delta}$ subset of $X$ ). Following a well-established terminology, we will say that a property of elements of $X$ is typical if the set of all $x \in X$ satisfying it is comeager in $X$, and that a property is atypical if its negation is typical. In this paper, we obtain among other things the following results, for any $M>1$.

- An SOT*-typical $T \in \mathfrak{B}_{M}(\mathcal{H})$ is (topologically) weakly mixing but not mixing.

- An SOT*-typical $T \in \mathfrak{B}_{M}(\mathcal{H})$ is densely distributionally chaotic.

- An SOT*-typical $T \in \mathrm{HC}_{M}(\mathcal{H})$ has no eigenvalues and admits no non-trivial invariant measure. In particular, chaoticity and $\mathcal{U}$-frequent hypercyclicity are atypical properties of hypercyclic operators (in the SOT* sense).

Admittedly, there results are not that surprising (except, perhaps, the one concerning $\mathcal{U}$-frequent hypercyclicity). Still, they do sketch the landscape, and they explain in some sense why some results in the area turn out to be (or are likely to be) harder to prove than 
some others. For example, the fact that topological weak mixing is a typical property seems to prevent the use of "soft" arguments for establishing the existence of hypercyclic operators which are not weakly mixing; and indeed, the proofs in [18 and [6] rely on rather technical arguments.

On the other hand, something more unexpected happens if one restricts oneself to the class of operators of the form $T=D+B$, where $D$ is a diagonal operator and $B$ is a weighted unilateral backward shift (with respect to some fixed orthonormal basis of $\mathcal{H}$ ):

- in the SOT sense, most "diagonal plus shift" hypercyclic operators are ergodic and yet have only countably many unimodular eigenvalues. In particular, there exist operators on $\mathcal{H}$ which are ergodic but admit no Gaussian ergodic measure with full support.

This solves a rather intriguing question, which seems to go back to Flytzanis' paper [22 and has been very much in the air in the last few years.

As for the "individual" viewpoint, we will elaborate on the kind of operators constructed by the third named author in [39]. Recall that the main result of [39] is the existence of chaotic operators on $\ell_{p}$ spaces which are not $\mathcal{U}$-frequently hypercyclic. In the present paper, we describe a general scheme allowing to produce, among other things,

- operators on $\mathcal{H}$ which are chaotic and frequently hypercyclic but not ergodic;

- operators on $\mathcal{H}$ which are chaotic and $\mathcal{U}$-frequently hypercyclic but not frequently hypercyclic.

These are the first examples of such kinds of operators living on a Hilbert space, even if one dispenses with the chaoticity assumption: no examples of frequently hypercyclic nonergodic operators, nor of $\mathcal{U}$-frequently hypercyclic non frequently hypercyclic operators living on a Hilbert space were known before. As already mentioned, up to now the only available examples were operators acting on $c_{0}$.

Moreover, our constructions will also enable us to improve the main result of [39] by showing that there exist

- operators on $\mathcal{H}$ which are chaotic and topologically mixing but not $\mathcal{U}$-frequently hypercyclic.

One point is especially worth mentioning regarding these results: it is not at all accidental that all the operators we construct turn out to be chaotic. On the contrary, our proofs rely heavily on new criteria for frequent hypercyclicity and $\mathcal{U}$-frequent hypercyclicity (stronger than the usual ones), in which the periodic points play a central role.

In this part of the paper, our constructions and arguments are rather technical, and it is not at all clear that they could be by-passed by suitable Baire category arguments. In fact, as already suggested above, there are reasons to believe that technicalities are unavoidable here: since $\mathcal{U}$-frequent hypercyclicity, frequent hypercyclicity and ergodicity are atypical properties, it seems difficult to distinguish them using simply the Baire category theorem; unless one restricts oneself to some cleverly chosen special class of operators, which has yet to be found.

The basic questions we address in this paper can also be considered from a third point of view, which is that of descriptive set theory. Indeed, once it is known (by any argument) that two properties of linear operators are not the same, i.e. that two classes of operators are distinct, it is natural to wonder if a stronger conclusion might hold true, namely 
that these classes do not have the same complexity in the sense of descriptive set theory. More generally, it can be a quite interesting problem to determine the exact descriptive complexity of a given class of operators. In this paper, we do so for chaotic operators and for topologically mixing operators. We also obtain a partial result for $\mathcal{U}$-frequently hypercyclic operators, whose proof relies on our general scheme for constructing operators with special properties.

We finish this overview by pointing out that the difficulty of the existence results presented in this paper is specifically connected with the linear setting, and that the corresponding results are essentially trivial if one moves over to the broader setting of Polish dynamical systems (i.e. dynamical systems of the form $(X, T)$, where $T$ is a continuous self-map of a Polish space $X$; see for instance the book [24], as well as [17] for more on linear systems as special cases of Polish systems). Indeed, extending the definitions of frequent and $\mathcal{U}$-frequent hypercyclicity to the Polish setting in the obvious way, it is not difficult to see that frequently hypercyclic non-ergodic Polish dynamical systems do exist, as well as $\mathcal{U}$-frequently hypercyclic Polish dynamical systems which are not frequently hypercyclic. Here are two simple examples.

First, consider an irrational rotation $R$ of the unit circle $\mathbb{T}$, and denote by $m$ the normalized Lebesgue measure on $\mathbb{T}$. Then $(\mathbb{T}, \mathcal{B}, m ; R)$ is an ergodic dynamical system for which all points are frequently hypercyclic. Let $C$ be a compact subset of $\mathbb{T}$ which has empty interior and is such that $m(C)>0$, and consider the set $X:=\mathbb{T} \backslash \bigcup_{n \in \mathbb{N}} R^{-n}(C)$. Then $X$ is a dense $G_{\delta}$ subset of $\mathbb{T}$ which is $R$-invariant, and $(X, R)$ is thus a frequently hypercyclic Polish dynamical system. But, as $m(X)=0$ (by ergodicity) and $R$ is uniquely ergodic, $(X, R)$ admits no invariant measure at all. This shows in particular the existence of frequently hypercyclic Polish dynamical systems which are not ergodic. This example is due to B. Weiss (private communication).

Now, consider a frequently hypercyclic operator $T$ on $\mathcal{H}$. Then, the set $\operatorname{UFHC}(T)$ of all $\mathcal{U}$-frequently hypercyclic vectors for $T$ is comeager in $\mathcal{H}$ ([9], see also [14]), whereas the set $\operatorname{FHC}(T)$ of frequently hypercyclic vectors for $T$ is meager $(\mathbf{4 0},[\mathbf{9}])$. Hence $\operatorname{UFHC}(T) \backslash$ $\operatorname{FHC}(T)$ is comeager in $\mathcal{H}$. Let $G$ be a dense $G_{\delta}$ subset of $\mathcal{H}$ contained in $\operatorname{UFHC}(T) \backslash$ $\operatorname{FHC}(T)$, and set $X:=\bigcap_{n \geq 0} T^{-n}(G)$. Then $X$ is a dense $G_{\delta}$ subset of $\mathcal{H}$ which is $T$ invariant, so that $(X, T)$ is a Polish dynamical system. Since $X$ is still contained in $\operatorname{UFHC}(T) \backslash \operatorname{FHC}(T)$, all points of $X$ are $\mathcal{U}$-frequently hypercyclic for $T$ but none of them is frequently hypercyclic. In particular, the Polish dynamical system $(X, T)$ is $\mathcal{U}$-frequently hypercyclic but not frequently hypercyclic.

1.2. Background and notations. - In this subsection, we recall some well-known definitions, referring to [7] or [31] for more details. We also fix some notations that will be used throughout the paper.

1.2.1. Transitivity, mixing and weak mixing. - If $T \in \mathfrak{B}(\mathcal{H})$, we set, for any subsets $A, B$ of $\mathcal{H}$,

$$
\mathcal{N}_{T}(A, B):=\left\{i \in \mathbb{N} ; T^{i}(A) \cap B \neq \emptyset\right\} .
$$

It is well-known that $T$ is hypercyclic if and only if it is topologically transitive, i.e. $\mathcal{N}_{T}(U, V) \neq \emptyset$ for all open sets $U, V \neq \emptyset$. A stronger property is topological mixing: $T$ is topologically mixing if all sets $\mathcal{N}_{T}(U, V)$ are cofinite rather than just non-empty; that is, for any pair $(U, V)$ of non-empty open sets in $\mathcal{H}$, one has $T^{i}(U) \cap V \neq \emptyset$ for all but finitely many $i \in \mathbb{N}$. In between transitivity and mixing is topological weak mixing: an operator 
$T \in \mathfrak{B}(\mathcal{H})$ is topologically weakly mixing if $T \times T$ is hypercyclic on $\mathcal{H} \times \mathcal{H}$; in other words, if $\mathcal{N}_{T}\left(U_{1}, V_{1}\right) \cap \mathcal{N}_{T}\left(U_{2}, V_{2}\right) \neq \emptyset$ for all non-empty open sets $U_{1}, V_{1}, U_{2}, V_{2} \subseteq \mathcal{H}$. We set

$$
\begin{aligned}
\operatorname{TWMIX}(\mathcal{H}) & :=\{\text { topologically weakly mixing operators on } \mathcal{H}\}, \\
\operatorname{TMIX}(\mathcal{H}) & :=\{\text { topologically mixing operators on } \mathcal{H}\}
\end{aligned}
$$

so that

$$
\operatorname{TMIX}(\mathcal{H}) \subseteq \operatorname{TWMIX}(\mathcal{H}) \subseteq \operatorname{HC}(\mathcal{H}) .
$$

It is easy to see (for example by considering weighted backward shifts) that the inclusion $\operatorname{TMIX}(\mathcal{H}) \subseteq \operatorname{TWMIX}(\mathcal{H})$ is proper. As already mentioned, the inclusion TWMIX $\subseteq$ HC is also proper: this was first proved by De La Rosa and Read in $\mathbf{1 8}$ for operators living on a suitably manufactured Banach space, and then in [6 for Hilbert space operators. Recall also that, according to a nice result of Bès and Peris [11, the topologically weakly mixing operators are exactly those satisfying the so-called Hypercyclicity Criterion.

1.2.2. Chaos. - An operator $T \in \mathfrak{B}(\mathcal{H})$ is said to be chaotic in the sense of Devaney if it is hypercyclic and its periodic points are dense in $\mathcal{H}$ (in the linear setting, hypercyclicity automatically implies sensitive dependence on the initial conditions). We set

$$
\mathrm{CH}(\mathcal{H}):=\{\text { chaotic operators on } \mathcal{H}\} .
$$

It is not completely obvious, but nonetheless true, that chaotic operators are topologically weakly mixing (see e.g. [7, Ch. 4]). In other words,

$$
\mathrm{CH}(\mathcal{H}) \subseteq \operatorname{TWMIX}(\mathcal{H}) .
$$

1.2.3. Ergodic-theoretic properties. - In this paper, the word "measure" will always mean "Borel probability measure". A measure $\mu$ on $\mathcal{H}$ is invariant for some operator $T \in \mathfrak{B}(\mathcal{H})$ if $\mu \circ T^{-1}=\mu$; and an invariant measure $\mu$ for $T$ is non-trivial if $\mu \neq \delta_{0}$ (note that the point mass $\delta_{0}$ is invariant for any $T \in \mathfrak{B}(\mathcal{H})$ since $\left.T(0)=0\right)$. Also, a measure $\mu$ on $\mathcal{H}$ is said to have full support if $\mu(V)>0$ for every non-empty open set $V$. This means exactly that the topological support of $\mu$ is equal to the whole space $\mathcal{H}$.

An operator $T \in \mathfrak{B}(\mathcal{H})$ is said to be ergodic if it admits an invariant measure $\mu$ with full support with respect to which it is ergodic, i.e. such that for every Borel subset $B$ of $\mathcal{H}$ satisfying $T^{-1} B=B$, we have $\mu(B)=0$ or 1 . If the measure $\mu$ can be taken to be Gaussian, we say that $T$ is ergodic in the Gaussian sense, or G-ergodic. The operator $T$ is said to be mixing if it admits an invariant measure $\mu$ with full support with respect to which it is strongly mixing, i.e. such that $\mu\left(A \cap T^{-n}(B)\right) \rightarrow \mu(A) \mu(B)$ for any Borel sets $A, B \subseteq \mathcal{H}$. Mixing in the Gaussian sense is defined as expected. The corresponding notations are the following:

$$
\begin{aligned}
\operatorname{ERG}(\mathcal{H}) & :=\{\text { ergodic operators on } \mathcal{H}\} \\
\operatorname{G-ERG}(\mathcal{H}) & :=\{\text { operators on } \mathcal{H} \text { which are ergodic in the Gaussian sense }\} \\
\operatorname{MIX}(\mathcal{H}) & :=\{\text { mixing operators on } \mathcal{H}\} \\
\operatorname{G-MIX}(\mathcal{H}) & :=\{\text { operators on } \mathcal{H} \text { which are mixing in the Gaussian sense }\}
\end{aligned}
$$

Since the measures involved are required to have full support, it is obvious by definition that ergodic operators are hypercyclic and that mixing operators are topologically mixing. That is,

$$
\operatorname{ERG}(\mathcal{H}) \subseteq \operatorname{HC}(\mathcal{H}) \quad \text { and } \quad \operatorname{MIX}(\mathcal{H}) \subseteq \operatorname{TMIX}(\mathcal{H})
$$


These inclusions are proper: for example, if $B$ is any compact weighted backward shift on the Hilbert space $\ell_{2}(\mathbb{N})$, the operator $T=I+B$ is topologically mixing (see [7] or [31]) but not ergodic: its spectrum is reduced to the point $\{1\}$, so that it is by 48 not even $\mathcal{U}$-frequently hypercyclic. We also set

$$
\begin{aligned}
\operatorname{INV}(\mathcal{H}) & :=\{T \in \mathfrak{B}(\mathcal{H}) \text { admitting a non-trivial invariant measure }\} \\
\operatorname{INV}_{f}(\mathcal{H}) & :=\{T \in \mathfrak{B}(\mathcal{H}) \text { admitting an invariant measure with full support }\} \\
\operatorname{G-INV}_{f}(\mathcal{H}) & :=\{T \in \mathfrak{B}(\mathcal{H}) \text { admitting a Gaussian invariant measure with full support }\} .
\end{aligned}
$$

Thus, for example, any operator admitting a non-zero periodic point belongs to $\operatorname{INV}(\mathcal{H})$, and any chaotic operator lies in $\operatorname{INV}_{f}(\mathcal{H})$. So we have

$$
\mathrm{CH}(\mathcal{H}) \subseteq \operatorname{INV}_{f}(\mathcal{H}) \cap \mathrm{HC}(\mathcal{H})
$$

This inclusion cannot be reversed: there exist even $G$-ergodic operators on $\mathcal{H}$ which are not chaotic (see [3] or [7, Section 6.5]).

At this point, we would like to stress that in the present paper, we will mostly focus on invariant measures which are not required to be Gaussian. This is a true change of point of view compared with earlier works on the ergodic theory of linear dynamical systems (see for instance, among other works, [22], 4, [5], 7] ...), where Gaussian measures play a central role. But in retrospect, this is in fact quite natural: as we shall see in Section 4 , it turns out that within a certain natural class of upper-triangular operators on $\mathcal{H}$, the ergodic operators which are not ergodic in the Gaussian sense are typical. Note also that non-Gaussian measures already played an essential role in such works as [41, 25] or [28] for instance.

1.2.4. Frequent hypercyclicity and $\mathcal{U}$-frequent hypercyclicity. - Let $T \in \mathfrak{B}(\mathcal{H})$. For any $x \in \mathcal{H}$ and $B \subseteq \mathcal{H}$, set

$$
\mathcal{N}_{T}(x, B):=\left\{i \in \mathbb{N} ; T^{i} x \in B\right\} .
$$

The operator $T$ is said to be frequently hypercyclic if there exists some $x \in \mathcal{H}$ such that, for any open set $V \neq \emptyset$, the set $\mathcal{N}_{T}(x, V)$ has positive lower density; and $T$ is $\mathcal{U}$-frequently hypercyclic if there is some $x \in \mathcal{H}$ such that all sets $\mathcal{N}_{T}(x, V)$ has positive upper density.

Frequent hypercyclicity was introduced in $[\mathbf{4}$ and rather extensively studied since then (for instance in [9], 13, 27, 29, [39, [48] ...). The study of upper-frequent hypercyclicity is more recent. This notion was introduced by Shkarin in 48. Until the last few years, it has perhaps been unfairly considered as somehow "less interesting", probably because of a lack of examples or results exhibiting truly different behaviors between frequently and $\mathcal{U}$-frequently hypercyclic operators. However, despite a formal similarity in the definitions, there are some important differences between frequent and $\mathcal{U}$-frequent hypercyclicity. For example, the set of frequently hypercyclic vectors for a frequently hypercyclic operator $T$ on $\mathcal{H}$ is always meager in $\mathcal{H}$ ([40], [9]) whereas if $T$ is a $\mathcal{U}$-frequently hypercyclic operator on $\mathcal{H}$, the set of all $\mathcal{U}$-frequently hypercyclic vectors for $T$ is comeager in $\mathcal{H}$ ([9]; see also [14] for more along these lines). This may lead to believe that $\mathcal{U}$-frequent hypercyclicity is a typical property while frequent hypercyclicity is not; which is in fact a wrong intuition: as we shall see, both properties are atypical. Yet, these properties are not equivalent: Bayart and Rusza exhibited in [9] examples of $\mathcal{U}$-frequently hypercyclic operators on $c_{0}$ which are not frequently hypercyclic. The notion of $\mathcal{U}$-frequent hypercyclicity was further studied by Bonilla and Grosse-Erdmann in [14], and it plays an important role in the present paper. 
One can go one step further and study $\mathcal{F}$-hypercyclic operators, for a given family $\mathcal{F}$ of subsets of $\mathbb{N}$ (see in particular $\mathbf{1 2}$ and [14]); but we will not follow this quite interesting route here. As for the notations, we set

$$
\begin{aligned}
\operatorname{FHC}(\mathcal{H}) & :=\{\text { frequently hypercyclic operators on } \mathcal{H}\} \\
\operatorname{UFHC}(\mathcal{H}) & :=\{\mathcal{U} \text {-frequently hypercyclic operators on } \mathcal{H}\} .
\end{aligned}
$$

Although the definitions of frequent hypercyclicity and $\mathcal{U}$-frequent hypercyclicity make no explicit reference to measures, they have a partly "metrical" flavour; and indeed, invariant measures are quite relevant here. First, it follows easily from the pointwise ergodic theorem that ergodic operators are frequently hypercyclic. Moreover, it is shown in [29] that $\mathcal{U}$-frequently hypercyclic operators on $\mathcal{H}$ admit invariant measures with full support (this is in fact true for operators living on any reflexive Banach space). So we have

$$
\operatorname{ERG}(\mathcal{H}) \subseteq \operatorname{FHC}(\mathcal{H}) \subseteq \operatorname{UFHC}(\mathcal{H}) \subseteq \mathrm{HC}(\mathcal{H}) \cap \operatorname{INV}_{f}(\mathcal{H})
$$

The inclusion $\operatorname{UFHC}(\mathcal{H}) \subseteq \operatorname{HC}(\mathcal{H}) \cap \operatorname{INV}_{f}(\mathcal{H})$ is proper: as already mentioned, it is proved in 39 that there exist even chaotic operators on $\mathcal{H}$ which are not $\mathcal{U}$-frequently hypercyclic. As we shall see in Section 6 (Theorems 6.22 and 6.29), the other two inclusions are also proper.

1.2.5. Properties related to eigenvalues. - If $T \in \mathfrak{B}(\mathcal{H})$, we denote by $\mathcal{E}(T)$ the set of all unimodular eigenvectors of $T$, i.e. eigenvectors $x$ of $T$ whose associated eigenvalue $\lambda(x)$ has modulus 1 . We say that $T$ has a spanning set of unimodular eigenvectors if $\overline{\operatorname{span}} \mathcal{E}(T)=\mathcal{H}$; and that $T$ has a perfectly spanning set of unimodular eigenvectors if for any countable set $D \subseteq \mathbb{T}$, where $\mathbb{T}$ denotes the unit circle in $\mathbb{C}$, we have $\overline{\operatorname{span}}\{x \in \mathcal{E}(T) ; \lambda(x) \notin D\}=\mathcal{H}$. The notations are as follows:

$$
\begin{aligned}
\operatorname{SPAN}(\mathcal{H}) & :=\{T \in \mathfrak{B}(\mathcal{H}) \text { with spanning unimodular eigenvectors }\} \\
\operatorname{PSPAN}(\mathcal{H}) & :=\{T \in \mathfrak{B}(\mathcal{H}) \text { with perfectly spanning unimodular eigenvectors }\} .
\end{aligned}
$$

We also define

$$
\begin{aligned}
& \operatorname{NEV}(\mathcal{H}):=\{T \in \mathfrak{B}(\mathcal{H}) \text { with no eigenvalues }\} \\
& \operatorname{CEV}(\mathcal{H}):=\{T \in \mathfrak{B}(\mathcal{H}) \text { with only countably many eigenvalues }\} .
\end{aligned}
$$

Properties of unimodular eigenvectors of an operator $T \in \mathfrak{B}(\mathcal{H})$ are closely related to those of invariant Gaussian measures for $T$ (see for instance [4, [5], [7]): indeed, it turns out that in fact

$$
\operatorname{SPAN}(\mathcal{H})=\operatorname{G-INV}_{f}(\mathcal{H}) \quad \text { and } \quad \operatorname{PSPAN}(\mathcal{H})=\operatorname{G-ERG}(\mathcal{H})
$$

so that there was in fact no need for introducing new notations. However, things are not that neat on arbitrary Banach spaces: see [5], 7] or [8. This is one of the advantages of working on Hilbert spaces, or at least on "nice" Banach spaces.

1.2.6. Distributional chaos. - Distributional chaos was first defined by Schweitzer and Smítal in [49], under the name strong chaos, for self-maps of a compact interval, and several definitions have been proposed afterwards in the context of general metric spaces. There is no need to recall these definitions here, because things simplify greatly in the linear setting: as shown in [10], an operator $T \in \mathfrak{B}(\mathcal{H})$ is distributionally chaotic if and 
only if it admits a distributionally irregular vector, i.e. a vector $x \in \mathcal{H}$ for which there exist two sets $A$ and $B$ of integers, both of upper density 1 in $\mathbb{N}$, such that

$$
\lim _{\substack{i \rightarrow \infty \\ i \in A}}\left\|T^{i} x\right\|=0 \quad \text { and } \quad \lim _{\substack{i \rightarrow \infty \\ i \in B}}\left\|T^{i} x\right\|=\infty .
$$

The corresponding notation is the following one:

$\operatorname{DCH}(\mathcal{H}):=\{$ distributionally chaotic operators on $\mathcal{H}\}$.

A related class is that of densely distributionally chaotic operators, i.e. of operators which admit a dense set of distributionally irregular vectors. We use the following notation for this set:

$$
\operatorname{DDCH}(\mathcal{H}):=\{\text { densely distributionally chaotic operators on } \mathcal{H}\} .
$$

Obviously, $\operatorname{DDCH}(\mathcal{H}) \subseteq \operatorname{DCH}(\mathcal{H})$.

1.2.7. The parameter $c(T)$. - Ergodicity and distributional chaos are closely related to a natural parameter introduced in [29]. This quantity $c(T) \in[0,1]$ is associated to any hypercyclic operator $T$, and essentially represents the maximal frequency with which the orbit of a hypercyclic vector for $T$ can visit a ball in $\mathcal{H}$ centered at 0 . The precise definition is as follows: for any $\alpha>0$, we have

$$
c(T)=\sup _{x \in H C(T)} \overline{\operatorname{dens}} \mathcal{N}_{T}(x, B(0, \alpha)) .
$$

It is shown in 29 that in fact $\overline{\operatorname{dens}} \mathcal{N}_{T}(x, B(0, \alpha))=c(T)$ for a comeager set of vectors $x \in H C(T)$; so we have in particular (for any $\alpha>0$ )

$$
c(T)=\sup \left\{c \geq 0 ; \overline{\operatorname{dens}}\left(\mathcal{N}_{T}(x, B(0, \alpha))\right) \geq c \text { for a comeager set of } x \in H C(T)\right\} .
$$

Note also that $c(T)>0$ if the operator $T$ is $\mathcal{U}$-frequently hypercyclic.

The last class of operators which we introduce is that of operators $T \in \mathrm{HC}(\mathcal{H})$ such that $c(T)$ is maximal:

$$
c \operatorname{MAX}(\mathcal{H}):=\{T \in \operatorname{HC}(\mathcal{H}) ; c(T)=1\} .
$$

Since, as proved in [10, the set of all distributionally irregular vectors for a given operator $T \in \operatorname{DDCH}(\mathcal{H})$ is comeager in $\mathcal{H}$, it is clear that $\operatorname{DDCH}(\mathcal{H}) \cap \mathrm{HC}(\mathcal{H}) \subseteq c \operatorname{MAX}(\mathcal{H})$. Moreover, it is shown in [29] (more accurately, half in [9] and half in [29]) that ergodic operators are densely distributionally chaotic: $\operatorname{ERG}(\mathcal{H}) \subseteq \operatorname{DDCH}(\mathcal{H})$. So we have

$$
\operatorname{ERG}(\mathcal{H}) \subseteq \operatorname{DDCH}(\mathcal{H}) \cap \mathrm{HC}(\mathcal{H}) \subseteq c \operatorname{MAX}(\mathcal{H}) .
$$

It is left as an open question in $2 \mathbf{2 9}$ to determine whether $\operatorname{HC}(\mathcal{H}) \cap \operatorname{INV}_{f}(\mathcal{H}) \subseteq$ $c \operatorname{MAX}(\mathcal{H})$, or whether at least $\operatorname{FHC}(\mathcal{H}) \subseteq c \operatorname{MAX}(\mathcal{H})$. It follows from the proof of the main result of [39] that the first inclusion does not hold true: the chaotic operator $T$ constructed there belongs to $\mathrm{HC}(\mathcal{H}) \cap \operatorname{INV}_{f}(\mathcal{H})$ (as does any chaotic operator on $\mathcal{H}$ ) but satisfies $c(T)=0$. However this operator is not frequently hypercyclic, and so the examples of [39] do not disprove the second inclusion. We will nonetheless show in the present paper that this inclusion does not hold true either (Theorem 6.22).

1.2.8. A last notation. - If $\Gamma(\mathcal{H})$ is a class of operators on $\mathcal{H}$, then, for any $M>0$, we set

$$
\Gamma_{M}(\mathcal{H}):=\{T \in \Gamma(\mathcal{H}) ;\|T\| \leq M\} .
$$


1.3. Organization of the paper. - In Section 2, we recall a few basic facts concerning the topologies SOT and SOT*, and then prove some "typicality" results in the spaces $\mathfrak{B}_{M}(\mathcal{H})$ and $\mathrm{HC}_{M}(\mathcal{H}), M>1$ with respect to the Strong* Operator Topology SOT*. These results can be summarized as follows: for any $M>1$, an $\mathrm{SOT}^{*}$-typical $T \in \mathrm{HC}_{M}(\mathcal{H})$ is topologically weakly mixing but not mixing (Propositions 2.16 and 2.17), has no eigenvalues (Corollary 2.23), admits no non-trivial invariant measure (Theorem 2.29), but is densely distributionally chaotic (Proposition 2.37).

Section 3 is a digression, in which we discuss the descriptive complexity of some of the families of operators introduced above. We show that for any $M>1, \operatorname{TMIX}_{M}(\mathcal{H})$ and $\mathrm{CH}_{M}(\mathcal{H})$ are Borel subsets of $\left(\mathfrak{B}_{M}(\mathcal{H}), \mathrm{SOT}^{*}\right)$ of class exactly $\boldsymbol{\Pi}_{3}^{0}$, aka $F_{\sigma \delta}$ (Propositions 3.2 and [3.4), whereas $\operatorname{UFHC}_{M}(\mathcal{H})$ is Borel of class at most $\boldsymbol{\Pi}_{4}^{0}$, and neither $\boldsymbol{\Pi}_{3}^{0}$ nor $\boldsymbol{\Sigma}_{3}^{0}$ (Corollary 3.12). We also show that some rather natural classes of operators defined by dynamical properties are non-Borel in $\mathfrak{B}_{M}(\mathcal{H})$; for example, the family of all operators $T \in \mathfrak{B}_{M}(\mathcal{H})$ admitting a hypercyclic restriction to an invariant subspace is non-Borel (Proposition [3.23), as well as the family of distributionally chaotic operators $T \in \mathfrak{B}_{M}(\mathcal{H})$ (Proposition 3.28). In contrast, the class of densely distributionally chaotic operators $T \in \mathfrak{B}_{M}(\mathcal{H})$ is $G_{\delta}$ (Proposition 2.37).

In Section 4, we consider ergodicity properties of upper triangular operators, this time with respect to the Strong Operator Topology SOT. We show first that for any $M>1$, an SOT-typical upper triangular operator $T \in \mathfrak{B}_{M}(\mathcal{H})$ is ergodic in the Gaussian sense (Proposition 4.4). On the other hand, we essentially show in Theorem 4.9 that an SOTtypical operator $T \in \mathfrak{B}_{M}(\mathcal{H})$ of the form "diagonal + backward shift" is ergodic but admits only countably many eigenvalues (and hence is ergodic but not ergodic in the Gaussian sense).

In Section 5, we prove several criteria for an operator $T$ to be $\mathcal{U}$-frequently hypercyclic or frequently hypercyclic. These criteria are rather different in spirit from the by now classical Frequent Hypercyclicity Criterion (13]) or from the more recent criteria which can be found in [12] or [14], since they rely explicitly on the existence of many periodic (or almost periodic) vectors for the operator $T$. However, our criterion for frequent hypercyclicity turns out to be stronger than the classical one: indeed, any operator satisfying the so-called Operator Specification Property also satisfies the assumptions of our criterion, whereas it is known [2 that operators satisfying the assumptions of the classical Frequent Hypercyclicity Criterion have the Operator Specification Property (but not conversely, see [2]). In the present paper, these criteria for $\mathcal{U}$-frequent hypercyclicity and frequent hypercyclicity are instrumental: we use them in order to simplify the proofs of several later results (more precisely, Corollary 5.11, Theorem 5.14 and Theorem 5.31 will be used in the proofs of the main results of Section (6). However, we believe that these criteria might be useful in other situations as well; and for this reason we have stated them in the setting of general Banach spaces.

In Section 6, which is by far the the most technical part of the paper, we develop a general machinery for producing hypercyclic operators with special properties. This machinery is very much inspired from the construction of [39], but things are done here in greater generality. Again, we hope that this approach could be useful to solve other questions as well. The operators we construct depend on a number of parameters, and we are able to determine in a rather precise way how the parameters influence on $\mathcal{U}$-frequent or frequent hypercyclicity, ergodicity or topological mixing. This allows us to produce the examples we are looking for. The main results we obtain are the existence of chaotic and 
frequently hypercyclic operators which are not in $c \operatorname{MAX}(\mathcal{H})$ and hence are not ergodic (Theorem 6.22), the existence of chaotic and $\mathcal{U}$-frequently hypercyclic operators which are not frequently hypercyclic (Theorem 6.29), and the existence of chaotic and topologically mixing operators which are not $\mathcal{U}$-frequently hypercyclic (Theorem 6.34).

We conclude the paper (Section 7) with a short list of possibly interesting questions.

Acknowledgements: We are grateful to Alfred Peris for interesting discussions, and also for kindly allowing us to reproduce here his proof of Theorem 5.36 .

\section{Typical properties of hypercyclic operators}

2.1. The strong and strong* topologies. - The Strong Operator Topology (SOT) on $\mathfrak{B}(\mathcal{H})$ is defined as follows: any $T_{0} \in \mathfrak{B}(\mathcal{H})$ has a neighborhood basis consisting of sets of the form

$$
\mathfrak{U}_{T_{0} ; x_{1}, \ldots, x_{s}, \varepsilon}:=\left\{T \in \mathfrak{B}(\mathcal{H}) ;\left\|\left(T-T_{0}\right) x_{i}\right\|<\varepsilon \quad \text { for } i=1, \ldots, s\right\}
$$

where $x_{1}, \ldots, x_{s} \in \mathcal{H}$ and $\varepsilon>0$. Thus, a net $\left(T_{i}\right) \subseteq \mathfrak{B}(\mathcal{H})$ tends to $T_{0} \in \mathfrak{B}(\mathcal{H})$ with respect to SOT if and only if $T_{i} x \rightarrow T_{0} x$ in norm for every $x \in H$.

The second (and perhaps a little less well-known) topology we will use is the Strong* Operator Topology (SOT*), which is the "self-adjoint" version of SOT. A basis of SOT*neighborhoods of $T_{0} \in \mathfrak{B}(\mathcal{H})$ is provided by the sets of the form

$$
\mathfrak{V}_{T_{0} ; x_{1}, \ldots, x_{s}, \varepsilon}:=\left\{T \in \mathfrak{B}(\mathcal{H}) ;\left\|\left(T-T_{0}\right) x_{i}\right\|<\varepsilon \text { and }\left\|\left(T-T_{0}\right)^{*} x_{i}\right\|<\varepsilon \quad \text { for } i=1, \ldots, s\right\}
$$

where $x_{1}, \ldots, x_{s} \in \mathcal{H}$ and $\varepsilon>0$. In other words, a net $\left(T_{i}\right)$ tends to $T_{0} \in \mathfrak{B}(\mathcal{H})$ with respect to SOT* if and only if $T_{i} \stackrel{\mathrm{SOT}}{\longrightarrow} T_{0}$ and $T_{i}^{*} \stackrel{\mathrm{SOT}}{\longrightarrow} T_{0}^{*}$. Obviously, SOT is coarser than SOT*, which is in turn coarser than the norm topology. The topologies induced by SOT and SOT* on any closed ball $\mathfrak{B}_{M}(\mathcal{H})$ are easily seen to be Polish, i.e. separable and completely metrizable (see e.g. [43, Section 4.6.2]). This will be of primary importance for us.

The following simple fact will be used repeatedly, sometimes without explicit mention.

Fact 2.1. - Let $M>0$. If $\mathfrak{B}(\mathcal{H})$ is endowed with either SOT or SOT*, then the map $(T, S) \mapsto T S$ is continuous from $\mathfrak{B}_{M}(\mathcal{H}) \times \mathfrak{B}_{M}(\mathcal{H})$ into $\mathfrak{B}(\mathcal{H})$. Consequently, for any fixed integer $n \geq 1$, the map $T \mapsto T^{n}$ is (SOT, SOT)-continuous and (SOT*, $\mathrm{SOT}^{*}$ )-continuous from $\mathfrak{B}_{M}(\mathcal{H})$ into $\mathfrak{B}(\mathcal{H})$.

Proof. - It is enough to check the statement involving the SOT topology; so we have to show that for any fixed vector $x \in \mathcal{H}$, the map $(T, S) \mapsto T S x$ is continuous from $\mathfrak{B}_{M}(\mathcal{H}) \times$ $\mathfrak{B}_{M}(\mathcal{H})$ into $\mathcal{H}$. Since the map $(T, S) \mapsto(T, S x)$ is continuous from $\mathfrak{B}_{M}(\mathcal{H}) \times \mathfrak{B}_{M}(\mathcal{H})$ into $\mathfrak{B}_{M}(\mathcal{H}) \times \mathcal{H}$, it suffices to check that the map $(T, u) \mapsto T u$ is continuous from $\mathfrak{B}_{M}(\mathcal{H}) \times \mathcal{H}$ into $\mathcal{H}$. Now, for any operators $T, T_{0} \in \mathfrak{B}_{M}(\mathcal{H})$ and any vectors $u, u_{0} \in \mathcal{H}$, we have

$$
\left\|T u-T_{0} u_{0}\right\|=\left\|T\left(u-u_{0}\right)+\left(T-T_{0}\right) u_{0}\right\| \leq M\left\|u-u_{0}\right\|+\left\|\left(T-T_{0}\right) u_{0}\right\| .
$$

The result follows immediately.

It is easy to deduce from this fact that $\mathrm{HC}_{M}(\mathcal{H})$ is SOT- $G_{\delta}$ in $\mathfrak{B}_{M}(\mathcal{H})$ for any $M>1$. Indeed, it follows from Fact 2.1 that if $A$ and $V$ are two non-empty subsets of $\mathcal{H}$ with $V$ open, then, for any fixed $n \geq 1$, the set

$$
\mathfrak{O}_{n ; A, V}:=\left\{T \in \mathfrak{B}_{M}(\mathcal{H}) ; T^{n}(A) \cap V \neq \emptyset\right\}
$$


is SOT-open in $\mathfrak{B}_{M}(\mathcal{H})$. Now, fix a countable basis of non-empty open sets $\left(V_{p}\right)_{p \geq 1}$ of $\mathcal{H}$, and observe that

$$
\mathrm{HC}_{M}(\mathcal{H})=\bigcap_{p, q \geq 1} \bigcup_{n \geq 1}\left\{T \in \mathfrak{B}_{M}(\mathcal{H}) ; T^{n}\left(V_{p}\right) \cap V_{q} \neq \emptyset\right\}=\bigcap_{p, q \geq 1} \bigcup_{n \geq 1} \mathfrak{O}_{n ; V_{p}, V_{q}} .
$$

This proves that $\mathrm{HC}_{M}(\mathcal{H})$ is $G_{\delta}$ in $\mathfrak{B}_{M}(\mathcal{H})$ with respect to SOT. Since the topology SOT* is finer than the topology $\mathrm{SOT}, \mathrm{HC}_{M}(\mathcal{H})$ is also $G_{\delta}$ in $\mathfrak{B}_{M}(\mathcal{H})$ with respect to SOT*. Thus, we may state:

Fact 2.2. - For any $M>1, \mathrm{HC}_{M}(\mathcal{H})$ is $\mathrm{SOT}-G_{\delta}$ and $\mathrm{SOT}^{*}-G_{\delta}$ in $\mathfrak{B}_{M}(\mathcal{H})$. Hence $\left(\mathrm{HC}_{M}(\mathcal{H}), \mathrm{SOT}\right)$ and $\left(\mathrm{HC}_{M}(\mathcal{H}), \mathrm{SOT}^{*}\right)$ are Polish spaces.

We now state a less immediate fact, which will be proved in Corollary 2.12 below. The corresponding (weaker) SOT statement can be found in [16], and its SOT* analogue undoubtedly would have been proved there if there had been any need to do so.

Fact 2.3. - For any $M>1, \mathrm{HC}_{M}(\mathcal{H})$ is dense in $\left(\mathfrak{B}_{M}(\mathcal{H})\right.$, $\left.\mathrm{SOT}^{*}\right)$. Hence, $\operatorname{HC}_{M}(\mathcal{H})$ is comeager in $\mathfrak{B}_{M}(\mathcal{H})$, both for the SOT and the SOT* topology.

Note that this result is indeed not completely trivial. For example, since the map $T \mapsto T^{*}$ is a homeomorphism of $\left(\mathfrak{B}_{M}(\mathcal{H}), \mathrm{SOT}^{*}\right)$, it immediately implies that a typical operator $T \in \mathfrak{B}_{M}(\mathcal{H})$ is hypercyclic and has a hypercyclic adjoint. In particular, this argument proves that there exist hypercyclic operators whose adjoint is also hypercyclic; which is a classical result of Salas obtained via an explicit construction in [44].

We deduce immediately from Fact 2.3 that the word "typical" has the same meaning in the whole of $\mathfrak{B}_{M}(\mathcal{H})$ or in the subclass $\operatorname{HC}_{M}(\mathcal{H})$ of hypercyclic operators in $\mathfrak{B}_{M}(\mathcal{H})$. This will be used repeatedly below, sometimes without explicit mention.

Fact 2.4. - Let $\Gamma(\mathcal{H})$ be a class of operators on $\mathcal{H}$, and let $M>1$. If $\Gamma_{M}(\mathcal{H})$ is a dense $G_{\delta}$ subset of $\left(\mathfrak{B}_{M}(\mathcal{H}), \tau\right)$, where $\tau$ is either SOT or SOT*, then $\Gamma_{M}(\mathcal{H}) \cap \mathrm{HC}(\mathcal{H})$ is a dense $G_{\delta}$ subset of $\left(\mathrm{HC}_{M}(\mathcal{H}), \tau\right)$ ). Conversely, if $\Gamma(\mathcal{H}) \subseteq \mathrm{HC}(\mathcal{H})$ and if $\Gamma_{M}(\mathcal{H})$ is dense $G_{\delta}$ (resp. comeager) in $\left(\mathrm{HC}_{M}(\mathcal{H}), \tau\right)$, then $\Gamma_{M}(\mathcal{H})$ is dense $G_{\delta}$ (resp. comeager) in $\left(\mathfrak{B}_{M}(\mathcal{H}), \tau\right)$.

Proof. - This is obvious: if $\Gamma_{M}(\mathcal{H})$ is dense $G_{\delta}$ in $\left(\mathfrak{B}_{M}(\mathcal{H}), \tau\right)$ then, by Fact 2.2, Fact 2.3 and the Baire category theorem, $\Gamma_{M}(\mathcal{H}) \cap \mathrm{HC}(\mathcal{H})$ is dense $G_{\delta}$ in $\mathfrak{B}_{M}(\mathcal{H})$, and hence in $\mathrm{HC}_{M}(\mathcal{H})$; and likewise for the converse. The "comeager" case follows from the " $G_{\delta}$ " case.

Here is a last fact concerning the topology SOT* that will be quite useful for us. Note that the corresponding statement for SOT is false. This is an important difference between the two topologies, which explains in particular why we will encounter some subsets of $\mathfrak{B}_{M}(\mathcal{H})$ which are $G_{\delta}$ with respect to SOT* and not with respect to SOT.

Fact 2.5. - Let us denote by $w$ the weak topology of $\mathcal{H}$. If $B$ is a bounded subset of $\mathcal{H}$, then the map $(T, x) \mapsto T x$ is continuous from $\left(\mathfrak{B}_{M}(\mathcal{H})\right.$, $\left.\operatorname{SOT}^{*}\right) \times(B, w)$ into $(\mathcal{H}, w)$.

Proof. - We have to show that for any fixed vector $e \in \mathcal{H}$, the map $(T, x) \mapsto\langle T x, e\rangle$ is continuous on $\left(\mathfrak{B}_{M}(\mathcal{H}), \mathrm{SOT}^{*}\right) \times(B, w)$. The key point is that one can separate $T$ from $x$ by writing $\langle T x, e\rangle=\left\langle x, T^{*} e\right\rangle$ (this trick would be useless, of course, if we were working with the SOT topology). 
Let $\left(T_{i}, x_{i}\right)$ be a net in $\mathfrak{B}(\mathcal{H}) \times B$ converging to some element $(T, x) \in \mathfrak{B}(\mathcal{H}) \times B$; that is, $T_{i} \stackrel{\mathrm{SOT}^{*}}{\longrightarrow} T$ and $x_{i} \stackrel{w}{\rightarrow} x$. Then

$$
\begin{aligned}
\left|\left\langle T_{i} x_{i}, e\right\rangle-\langle T x, e\rangle\right| & =\left|\left\langle x_{i}, T_{i}^{*} e\right\rangle-\left\langle x, T^{*} e\right\rangle\right| \\
& \leq\left|\left\langle x_{i}, T_{i}^{*} e-T^{*} e\right\rangle\right|+\left|\left\langle x_{i}-x, T^{*} e\right\rangle\right| .
\end{aligned}
$$

Since the net $\left(x_{i}\right)$ is bounded in norm and $x_{i} \stackrel{w}{\rightarrow} x$, this shows that $\left\langle T_{i} x_{i}, e\right\rangle \rightarrow\langle T x, e\rangle$.

2.1.1. Why SOT and SOT*? - There are other natural topologies on $\mathfrak{B}(\mathcal{H})$, most notably the operator norm topology, of course, and the Weak Operator Topology (WOT). The norm topology is not very well-suited for Baire category arguments, mainly because it is much too strong; in particular, the lack of separability seems unacceptable. The topology WOT is better behaved in this respect, being Polish on any closed ball $\mathfrak{B}_{M}(\mathcal{H})$. However, since we are interested in typical properties of hypercyclic operators, it seems better to consider topologies with respect to which $\operatorname{HC}_{M}(\mathcal{H})$ is comeager in $\mathfrak{B}_{M}(\mathcal{H})$ for any $M>1$. This is definitely not the case for WOT. Indeed, it is proved in $1 \mathbf{1 9}$ that a typical element of $\left(\mathfrak{B}_{1}(\mathcal{H})\right.$, WOT $)$ is unitary. It follows that a WOT-typical $T \in \mathfrak{B}_{M}(\mathcal{H})$ is a multiple of a unitary operator and hence not hypercyclic. Incidentally, the operator norm topology has the same "drawback", in an even stronger way: the hypercyclic operators (actually, even the cyclic operators) are in fact nowhere dense in $\mathfrak{B}(\mathcal{H})$; see [7, Section 2.5].

So we will consider neither the operator norm topology nor the Weak Operator Topology in this paper. Actually, when working in the whole of $\mathfrak{B}_{M}(\mathcal{H})$ or $\operatorname{HC}_{M}(\mathcal{H}), M>1$, we will always use SOT* rather than SOT. Indeed, with respect to SOT, a result from 21 gives a rather complete picture as far as typical properties are concerned: a typical element of $\left(\mathfrak{B}_{1}(\mathcal{H}), \mathrm{SOT}\right)$ is unitarily equivalent to the operator $B^{(\infty)}$, the countable direct $\ell_{2}$-sum of the unilateral backward shift $B$ on $\ell_{2}(\mathbb{N})$. It follows that for every $M>1$, the class of operators $T \in \mathrm{HC}_{M}(\mathcal{H})$ which are unitarily equivalent to $M B^{(\infty)}$ is comeager in $\left(\mathrm{HC}_{M}(\mathcal{H}), \mathrm{SOT}\right)$. Now, the dynamical properties of the operators $M B^{(\infty)}, M>1$ are quite strong and very well understood: these operators are mixing in the Gaussian sense (and hence ergodic and topologically mixing), densely distributionally chaotic, and they have nearly any other strong dynamical property one might think of. This explains why, when trying to determine which properties are typical within the class of all hypercyclic operators, we will use SOT* rather than SOT. The situation in this setting is more involved, and thus leads to more interesting results.

On the other hand, we will see that within specific subclasses of $\mathrm{HC}(\mathcal{H})$ consisting of upper triangular operators, the topology SOT becomes much more useful. This is not really surprising, since triangularity is not exactly a self-adjoint property.

2.2. How to prove density results. - For future reference, we state here a simple criterion for a class of operators to be dense in $\left(\mathfrak{B}_{M}(\mathcal{H}), \mathrm{SOT}\right)$ or $\left(\mathfrak{B}_{M}(\mathcal{H}), \mathrm{SOT}^{*}\right), M>0$. We will use it repeatedly in the rest of the paper.

Lemma 2.6. - Let $\Gamma(\mathcal{H})$ be a class of operators on $\mathcal{H}$, and let $M>0$. Let also $\left(e_{k}\right)_{k \geq 1}$ be an orthonormal basis of $\mathcal{H}$, and for each $r \geq 1$, denote by $\mathcal{H}_{r}$ the finite-dimensional subspace $\operatorname{span}\left[e_{k} ; 1 \leq k \leq r\right]$ of $\mathcal{H}$. Suppose that the following property holds true:

for every $r \geq 1$, every operator $A \in \mathfrak{B}\left(\mathcal{H}_{r}\right)$ with $\|A\|<M$ and every $\varepsilon>0$, there exists an operator $T \in \Gamma_{M}(\mathcal{H})$ such that

$$
\left\|(T-A) e_{k}\right\|<\varepsilon \quad \text { for } k=1, \ldots, r .
$$


Then $\Gamma_{M}(\mathcal{H})$ is dense in $\left(\mathfrak{B}_{M}(\mathcal{H}), \mathrm{SOT}\right)$. If (困) is replaced by its self-adjoint version

$$
\left\|(T-A) e_{k}\right\|<\varepsilon \quad \text { and } \quad\left\|(T-A)^{*} e_{k}\right\|<\varepsilon \quad \text { for } k=1, \ldots, r,
$$

then $\Gamma_{M}(\mathcal{H})$ is dense in $\left(\mathfrak{B}_{M}(\mathcal{H})\right.$, SOT $\left.^{*}\right)$.

Note that there is a slight abuse of notation in the statement of Lemma 2.6. we consider the operator $A \in \mathfrak{B}\left(\mathcal{H}_{r}\right)$ as an operator on $\mathcal{H}$ by identifying it with $P_{r}^{*} A P_{r}$, where $P_{r}$ : $\mathcal{H} \rightarrow \mathcal{H}_{r}$ is the orthogonal projection of $\mathcal{H}$ onto $\mathcal{H}_{r}$.

Proof. - We will prove the assertion concerning the SOT*-topology, the proof of the SOT statement being exactly the same. Fix $T_{0} \in \mathfrak{B}_{M}(\mathcal{H}), \varepsilon>0$, and $x_{1}, \ldots, x_{s} \in \mathcal{H}$. Without loss of generality, we can suppose that $\left\|T_{0}\right\|<M$. We are looking for an operator $T \in$ $\Gamma_{M}(\mathcal{H})$ such that

$$
\max _{1 \leq j \leq s} \max \left(\left\|\left(T-T_{0}\right) x_{j}\right\|,\left\|\left(T-T_{0}\right)^{*} x_{j}\right\|\right)<\varepsilon .
$$

Since $\|T\| \leq M$ for every $T \in \Gamma_{M}(\mathcal{H})$, and since every vector $x_{j}$ can be approximated by a finite linear combination of the basis vectors $e_{k}$, there exists an integer $r_{0} \geq 1$ sufficiently large such that (1) above holds true as soon as

$$
\max _{1 \leq k \leq r_{0}} \max \left(\left\|\left(T-T_{0}\right) e_{k}\right\|,\left\|\left(T-T_{0}\right)^{*} e_{k}\right\|\right)<\frac{\varepsilon}{2} .
$$

For each $r \geq r_{0}$, consider the operator $A_{r}=P_{r} T_{0} P_{r}$ Observe that $\left\|A_{r}\right\| \leq\left\|T_{0}\right\|<M$. By our assumption, there exists $T \in \Gamma(\mathcal{H})$ such that

$$
\max _{1 \leq k \leq r} \max \left(\left\|\left(T-A_{r}\right) e_{k}\right\|,\left\|\left(T-A_{r}\right)^{*} e_{k}\right\|\right)<\frac{\varepsilon}{2} .
$$

Now

$$
\left\|\left(T-T_{0}\right) e_{k}\right\| \leq\left\|\left(T-A_{r}\right) e_{k}\right\|+\left\|\left(P_{r} T_{0} P_{r}-T_{0}\right) e_{k}\right\|<\frac{\varepsilon}{2}+\left\|\left(P_{r}-I\right) T_{0} e_{k}\right\|
$$

and

$$
\left\|\left(T-T_{0}\right)^{*} e_{k}\right\|<\frac{\varepsilon}{2}+\left\|\left(P_{r}-I\right) T_{0}^{*} e_{k}\right\|
$$

for every $1 \leq k \leq r$. Since $\lim _{r \rightarrow \infty} P_{r}=I$ for the SOT topology, one can choose $r$ so large that

$$
\max _{1 \leq k \leq r_{0}} \max \left(\left\|\left(P_{r}-I\right) T_{0} e_{k}\right\|,\left\|\left(P_{r}-I\right) T_{0}^{*} e_{k}\right\|\right)<\varepsilon
$$

from which the conclusion of Lemma 2.6 follows.

Remark 2.7. - It is sometimes more convenient to endow $\mathcal{H}$ with an orthonormal basis $\left(f_{k}\right)_{k \in \mathbb{Z}}$ indexed by $\mathbb{Z}$ rather than by $\mathbb{N}$. In this case, the corresponding version of Lemma 2.6 reads as follows.

For each $r \geq 0$, denote by $\mathcal{H}_{r}$ the finite-dimensional subspace span $\left[f_{k} ;-r \leq k \leq r\right]$ of $\mathcal{H}$. Suppose that the following property holds true:

for every $r \geq 0$, every operator $A \in \mathfrak{B}\left(\mathcal{H}_{r}\right)$ with $\|A\|<M$ and every $\varepsilon>0$, there exists an operator $T \in \Gamma_{M}(\mathcal{H})$ such that

$$
\left\|(T-A) f_{k}\right\|<\varepsilon \quad \text { for } k=-r, \ldots, r .
$$

Then $\Gamma_{M}(\mathcal{H})$ is dense in $\left(\mathfrak{B}_{M}(\mathcal{H}), \mathrm{SOT}\right)$. If (囝) is replaced by its self-adjoint version

$$
\left\|(T-A) f_{k}\right\|<\varepsilon \text { and }\left\|(T-A)^{*} f_{k}\right\|<\varepsilon \quad \text { for } k=-r, \ldots, r,
$$

then $\Gamma_{M}(\mathcal{H})$ is dense in $\left(\mathfrak{B}_{M}(\mathcal{H}), \mathrm{SOT}^{*}\right)$. 
2.3. Construction of mixing operators, and density of G-MIX $(\mathcal{H})$. - In order to show that a property is typical, we first need to prove the density of the set of operators satisfying it. In this subsection, we show that the class $\operatorname{G-MIX}_{M}(\mathcal{H})$ is dense in $\left(\mathrm{HC}_{M}(\mathcal{H}), \mathrm{SOT}^{*}\right)$ for any $M>1$. This will be achieved by considering some perturbations of weighted unilateral or bilateral weighted shifts with respect to some orthonormal basis of $\mathcal{H}$. We show that these operators admit spanning eigenvector fields which are analytic in a neighborhood of the unit circle, and hence are mixing in the Gaussian sense. The precise statement we will use is the following.

Fact 2.8. - Let $T \in \mathfrak{B}(\mathcal{H})$. Assume that there exists a connected open set $\Omega \subseteq \mathbb{C}$ with $\Omega \cap \mathbb{T} \neq \emptyset$ and a family $\left(E_{i}\right)_{i \in I}$ of holomorphic maps, $E_{i}: \Omega \rightarrow \mathcal{H}$, such that $T E_{i}(\lambda)=\lambda E_{i}(\lambda)$ for every $i \in I$ and every $\lambda \in \Omega$, and $\overline{\operatorname{span}}\left\{E_{i}(\lambda) ; i \in I, \lambda \in \Omega\right\}=\mathcal{H}$. Then $T$ is mixing in the Gaussian sense.

Proof. - Recall that we denote by $\mathcal{E}(T)$ the set of all unimodular eigenvectors of $T$, and by $\lambda(x)$ the eigenvalue associated to $x \in \mathcal{E}(T)$. By [4, Th. 3.29], it is enough to show that for any Borel set $D \subseteq \mathbb{T}$ of Lebesgue measure 0, we have $\overline{\operatorname{span}}\{x \in \mathcal{E}(T) ; \lambda(x) \notin D\}=\mathcal{H}$. Let $y \in \mathcal{H}$ be orthogonal to the set $\{x \in \mathcal{E}(T) ; \lambda(x) \notin D\}$. Then $\left\langle y, E_{i}(\lambda)\right\rangle=0$ for every $i \in I$ and every $\lambda \in(\Omega \cap \mathbb{T}) \backslash D$. Since the functions $\left\langle y, E_{i}(\cdot)\right\rangle$ are holomorphic on $\Omega$, and since $(\Omega \cap \mathbb{T}) \backslash D$ certainly has an accumulation point in $\Omega$ (because $D$ has Lebesgue measure 0 ), it follows that $y$ is orthogonal to all vectors $E_{i}(\lambda), i \in I, \lambda \in \Omega$, and hence that $y=0$. This concludes the proof.

Remark 2.9. - The assumptions of Fact 2.8 imply that the operator $T$ is also chaotic: since the roots of unity contained in $\Omega$ have an accumulation point in $\Omega$, this follows as above from the identity principle for holomorphic functions.

Let us first consider perturbations of unilateral weighted shifts. Let $\left(e_{k}\right)_{k \geq 1}$ be an orthonormal basis of the Hilbert space $\mathcal{H}$, and let $\boldsymbol{\omega}=\left(\omega_{k}\right)_{k>1}$ be a unilateral weight sequence, i.e. a bounded sequence of positive real numbers. Let $r \geq 1$ be an integer, and let $A$ be an operator acting on the finite-dimensional space $\mathcal{H}_{r}=\operatorname{span}\left[e_{k} ; 1 \leq k \leq r\right]$. We define a bounded operator $B_{A, \omega}$ on $\mathcal{H}$ by setting

$$
B_{A, \omega} e_{k}= \begin{cases}A e_{k} & \text { for every } 1 \leq k \leq r \\ \omega_{k-r} e_{k-r} & \text { for every } k>r .\end{cases}
$$

Proposition 2.10. - Let $\boldsymbol{\omega}$ be a unilateral weight sequence, $r \geq 1$, and $A \in \mathfrak{B}\left(\mathcal{H}_{r}\right)$. Suppose that for every $1 \leq l \leq r$,

$$
R_{l}:=\liminf _{p \rightarrow \infty}\left(\omega_{p r+l} \cdots \omega_{r+l} \omega_{l}\right)^{1 / p}>\max (1,\|A\|) .
$$

Then the operator $B_{A, \omega}$ is mixing in the Gaussian sense. Besides, $B_{A, \omega}$ is also chaotic.

Proof. - Solving formally the equation $B_{A, \omega} x=\lambda x$, where $\lambda \in \mathbb{C}$ and $x=\left(x_{k}\right)_{k \geq 1} \in \mathbb{C}^{\mathbb{N}}$, one gets the following identities:

$$
(\lambda-A) P_{r} x=\sum_{l=1}^{r} \omega_{l} x_{l+r} e_{l} \quad \text { and } \quad \omega_{k} x_{k+r}=\lambda x_{k} \quad \text { for every } k>r .
$$


From this (setting $y:=P_{r} x$ ) we infer that the eigenvectors of $B_{A, \omega}$ associated to the eigenvalue $\lambda$ must be given by the formula

$$
E_{y}(\lambda)=y+\sum_{l=1}^{r} \frac{1}{\omega_{l}}\left\langle(\lambda-A) y, e_{l}\right\rangle\left(e_{r+l}+\sum_{p \geq 2} \frac{\lambda^{p-1}}{\omega_{(p-1) r+l} \cdots \omega_{r+l}} e_{p r+l}\right)
$$

where $y$ is a non-zero vector of $\mathcal{H}_{r}$. Conversely, if $y$ belongs to $\mathcal{H}_{r} \backslash\{0\}$ is such that the above formula makes sense, then $E_{y}(\lambda)$ is an eigenvector of $B_{A, \boldsymbol{\omega}}$ with associated eigenvalue $\lambda$. It follows that the complex number $\lambda$ is an eigenvalue of $B_{A, \boldsymbol{\omega}}$ as soon as

$$
\sum_{p \geq 2}\left|\frac{\lambda^{p-1}}{\omega_{(p-1) r+l} \ldots \omega_{l}}\right|^{2}<\infty \quad \text { for all } 1 \leq l \leq r,
$$

which holds true whenever $|\lambda|\left\langle R:=\min _{1 \leq l \leq r} R_{l}\right.$. In this case, the eigenvector field $E_{y}$ is well-defined and holomorphic on the open disk $D(0, R)$ for every $y \in \mathcal{H}_{r}$. Note that our assumption implies that $R>1$, so that the disk $D(0, R)$ contains $\mathbb{T}$.

By Fact 2.8 and Remark 2.9, in order to show that $B_{A, \omega}$ belongs to G-MIX $(\mathcal{H})$ and is chaotic, it suffices to check that the eigenvectors $E_{y}(\lambda), y \in \mathcal{H}_{r},|\lambda|<R$, span a dense subspace of $\mathcal{H}$. So let $u \in \mathcal{H}$ be such that $\left\langle E_{y}(\lambda), u\right\rangle=0$ for every $y \in \mathcal{H}_{r}$ and every $|\lambda|<R$. Writing $u$ as $u=\sum_{k \geq 1} u_{k} e_{k}$, this means that

$$
\langle y, u\rangle+\sum_{l=1}^{r} \frac{1}{\omega_{l}}\left\langle y,(\lambda-A)^{*} e_{l}\right\rangle\left(\bar{u}_{r+l}+\sum_{p \geq 2} \bar{u}_{p r+l} \frac{\lambda^{p-1}}{\omega_{(p-1) r+l} \cdots \omega_{r+l}}\right)=0
$$

for every $y \in \mathcal{H}_{r}$. It follows that each vector

$$
u+\sum_{l=1}^{r} \frac{1}{\omega_{l}}\left(u_{r+l}+\sum_{p \geq 2} u_{p r+l} \frac{\bar{\lambda}^{p-1}}{\omega_{(p-1) r+l} \ldots \omega_{r+l}}\right)(\lambda-A)^{*} e_{l}, \quad|\lambda|<R
$$

is orthogonal to $\mathcal{H}_{r}$, i.e. belongs to the closed linear span of the vectors $e_{k}, k>r$. In other words,

$$
P_{r} u+\sum_{l=1}^{r} \frac{1}{\omega_{l}}\left(u_{r+l}+\sum_{p \geq 2} u_{p r+l} \frac{\bar{\lambda}^{p-1}}{\omega_{(p-1) r+l} \cdots \omega_{r+l}}\right)(\lambda-A)^{*} e_{l}=0 .
$$

Consider now the open subset $\Omega=D(0, R) \backslash \sigma(A)$ of $\mathbb{C}$, where $\sigma(A)$ denotes the spectrum of $A$. Applying the operator $(\lambda-A)^{*-1}$ to the previous equation, we obtain that for every $\lambda \in \Omega:$

$$
(\lambda-A)^{*-1} P_{r} u=-\sum_{l=1}^{r} \frac{1}{\omega_{l}}\left(u_{r+l}+\sum_{p \geq 2} u_{p r+l} \frac{\bar{\lambda}^{p-1}}{\omega_{(p-1) r+l} \cdots \omega_{r+l}}\right) e_{l} .
$$

Since the expression on the right hand side of (3) defines an antiholomorphic map on $D(0, R)$ and since the disk $D(0, R)$ contains $\sigma(A)$ (recall that $R>\|A\|$ by assumption), it follows that the map $\lambda \mapsto(\lambda-A)^{*-1} P_{r} u$ extends antiholomorphically to the whole complex plane. But $(\lambda-A)^{*-1} P_{r} u \rightarrow 0$ as $|\lambda| \rightarrow \infty$, so the function $\lambda \mapsto(\lambda-A)^{*-1} P_{r} u$ must vanish identically on $\Omega$ by Liouville's Theorem, which is possible only if $P_{r} u=0$.

Going back to (3), the fact that $P_{r} u=0$ yields that $u_{p r+l}=0$ for every $1 \leq l \leq r$ and every $p \geq 2$, and that $u_{r+l}=0$ for every $1 \leq l \leq r$. Thus $u=0$, and this concludes the proof of Proposition 2.10. 
Remark 2.11. - The definition of the operator $B_{A, \omega}$ shows that every eigenvalue of $A$ is also an eigenvalue of $B_{A, \omega}$. This explains why the conditions $R_{l}>1$ for every $l=1, \ldots, r$ are not sufficient to ensure that $B_{A, \omega}$ be mixing in the Gaussian sense. Indeed, if $\lambda \in \sigma(A)$ is such that $|\lambda|>\max _{1 \leq l \leq r} R_{l}$, then $\lambda$ is an isolated eigenvalue of $B_{A, \omega}$ with $|\lambda|>1$, and this prevents $B_{A, \boldsymbol{\omega}}$ from being hypercyclic. This is to be compared with Remark 2.15 below.

From Proposition 2.10 we easily deduce a basic density result, which gives in particular the promised proof of Fact 2.3 above.

Corollary 2.12. - For every $M>1$, the class $\operatorname{G-MIX}_{M}(\mathcal{H}) \cap \mathrm{CH}_{M}(\mathcal{H})$ is dense in $\left(\mathrm{HC}_{M}(\mathcal{H}), \mathrm{SOT}^{*}\right)$.

Proof. - We are going to apply Lemma 2.6. So, let $\left(e_{k}\right)_{k \geq 1}$ be an orthonormal basis of $\mathcal{H}$, and let us fix $r \geq 1$, an operator $A \in \mathfrak{B}\left(\mathcal{H}_{r}\right)$ with $\|A\|<M$ and $\varepsilon>0$. Let also $\delta>0$ be a small positive number to be specified below. We define a weight sequence $\boldsymbol{\omega}=\left(\omega_{k}\right)_{k \geq 1}$ as follows:

$$
\omega_{k}= \begin{cases}\delta & \text { for every } 1 \leq k \leq r \\ M & \text { for every } k>r\end{cases}
$$

and we consider the associated operator $B_{A, \omega}$ acting on $\mathcal{H}$. Identifying $A$ with $P_{r}^{*} A P_{r} \in$ $\mathfrak{B}(\mathcal{H})$, we have for every $1 \leq k \leq r$ :

$$
B_{A, \omega} e_{k}=A e_{k} \quad \text { and } \quad B_{A, \omega}^{*} e_{k}=A^{*} e_{k}+\delta e_{k+r},
$$

so that $\left\|\left(B_{A, \omega}-A\right)^{*} e_{k}\right\|=\delta$. It follows that if $\delta<\varepsilon$, then

$$
\max _{1 \leq k \leq r} \max \left(\left\|\left(B_{A, \omega}-A\right) e_{k}\right\|,\left\|\left(B_{A, \omega}-A\right)^{*} e_{k}\right\|\right)<\varepsilon .
$$

The assumption of Proposition 2.10 is clearly satisfied, so that $B_{A, \omega}$ belongs to the class $\operatorname{G-MIX}(\mathcal{H}) \cap \mathrm{CH}(\mathcal{H})$.

To estimate the norm of $B_{A, \boldsymbol{\omega}}$, note that for every $x=\sum_{j \geq 1} x_{j} e_{j} \in H$, we have

$$
B_{A, \boldsymbol{\omega}} x=A P_{r} x+\sum_{k=1}^{r} x_{k+r} \delta e_{k}+\sum_{k>r} x_{k+r} M e_{k}
$$

so that

$$
\left\|B_{A, \omega} x\right\|^{2}=\left\|A P_{r} x+\delta \sum_{k=1}^{r} x_{k+r} e_{k}\right\|^{2}+M^{2} \sum_{k>r}\left|x_{k+r}\right|^{2} .
$$

Since $\|A\|<M$, it follows that $\left\|B_{A, \boldsymbol{\omega}}\right\|=M$ if $\delta>0$ is sufficiently small. So $B_{A, \boldsymbol{\omega}}$ belongs to G-MIX ${ }_{M}(\mathcal{H}) \cap \mathrm{CH}_{M}(\mathcal{H})$, and Lemma 2.6 now allows us to conclude the proof of Corollary 2.12.

Remark 2.13. - Corollary 2.12 does not state that G-MIX ${ }_{M}(\mathcal{H}) \cap \mathrm{CH}_{M}(\mathcal{H})$ is comeager in $\left(\mathrm{HC}_{M}(\mathcal{H}), \mathrm{SOT}^{*}\right)$. Indeed, we will prove below that $\mathrm{G}-\mathrm{MIX} M(\mathcal{H})$ and $\mathrm{CH}_{M}(\mathcal{H})$ are actually meager in $\left(\mathrm{HC}_{M}(\mathcal{H}), \mathrm{SOT}^{*}\right)$.

Let us now turn to a bilateral analogue of Proposition 2.10, which we state as Proposition 2.14 below. Let $\left(f_{k}\right)_{k \in \mathbb{Z}}$ be an orthonormal basis of the space $\mathcal{H}$, and let $\boldsymbol{\omega}=\left(\omega_{k}\right)_{k \in \mathbb{Z}}$ be a bilateral weight sequence, i.e. a bounded sequence of positive real numbers indexed 
by $\mathbb{Z}$. For any integer $r \geq 0$, we write $\mathcal{H}_{r}=\operatorname{span}\left[e_{k} ;|k| \leq r\right]$, and let $A \in \mathfrak{B}\left(\mathcal{H}_{r}\right)$ be a bounded operator on $\mathcal{H}_{r}$. We define a bounded operator $S_{A, \boldsymbol{\omega}}$ on $\mathcal{H}$ by setting

$$
S_{A, \omega} f_{k}= \begin{cases}A f_{k}+\omega_{k-(2 r+1)} f_{k-(2 r+1)} & \text { for every }|k| \leq r \\ \omega_{k-(2 r+1)} f_{k-(2 r+1)} & \text { for every }|k|>r .\end{cases}
$$

Proposition 2.14. - Let $\boldsymbol{\omega}$ be a bilateral weight sequence, $r \geq 1$ and $A \in \mathfrak{B}\left(\mathcal{H}_{r}\right)$. Suppose that for every $-r \leq l \leq r$,

$$
R_{l}:=\liminf _{p \rightarrow \infty}\left(\omega_{p(2 r+1)+l} \ldots \omega_{(2 r+1)+l}\right)^{1 / p}>1
$$

and

$$
r_{l}:=\limsup _{p \rightarrow \infty}\left(\omega_{l-p(2 r+1)} \ldots \omega_{l-(2 r+1)}\right)^{1 / p}<1 .
$$

Then $S_{A, \boldsymbol{\omega}}$ is mixing in the Gaussian sense and chaotic.

Proof. - The proof is so similar to that of Proposition 2.10 that we will not give it in detail. A complex number $\lambda$ is an eigenvalue of $S_{A, \omega}$ as soon as the series

$$
\sum_{p \geq 2}\left|\frac{\lambda^{p-1}}{\omega_{(p-1)(2 r+1)+l} \ldots \omega_{(2 r+1)+l}}\right|^{2} \text { and } \sum_{p \geq 1}\left|\frac{\omega_{l-p(2 r+1)} \cdots \omega_{l-(2 r+1)}}{\lambda^{p}}\right|^{2}
$$

are convergent for all $-r \leq l \leq r$. If we define $R:=\min _{-r \leq l \leq r} R_{l}$ and $r:=\max _{-r \leq l \leq r} r_{l}$, our assumption implies that $r<1<R$. Any complex number $\lambda$ belonging to the annulus $\{r<|\lambda|<R\}$ is an eigenvalue of $S_{A, \omega}$, and the eigenvectors of $S_{A, \omega}$ associated to $\lambda$ have the form

$$
\begin{aligned}
E_{y}(\lambda)=\sum_{l=-r}^{r} \frac{1}{\omega_{l}} & \left\langle(\lambda-A) y, e_{l}\right\rangle\left(e_{(2 r+1)+l}+\sum_{p \geq 2} \frac{\lambda^{p-1}}{\omega_{(p-1)(2 r+1)+l} \ldots \omega_{(2 r+1)+l}} e_{p(2 r+1)+l}\right. \\
& \left.+\sum_{p \geq 1} \frac{\omega_{l-p(2 r+1)} \ldots \omega_{l-(2 r+1)}}{\lambda^{p}} e_{-p(2 r+1)+l}\right)
\end{aligned}
$$

where $y \in \mathcal{H}_{r}$. Since $r<1<R$, the annulus $\{r<|\lambda|<R\}$ contains $\mathbb{T}$, and an argument similar to the one given in the proof of Proposition 2.10 show that $S_{A, \omega}$ belongs to $\operatorname{G-MIX}(H) \cap \mathrm{CH}_{M}(\mathcal{H})$. Indeed, if $u \in \mathcal{H}$ is such that $\left\langle E_{y}(\lambda), u\right\rangle=0$ for every $y \in \mathcal{H}_{r}$ and every $r<|\lambda|<R$, then

$$
\begin{aligned}
\sum_{l=-r}^{r} \frac{1}{\omega_{l}}\left(u_{(2 r+1)+l}\right. & +\sum_{p \geq 2} \frac{\bar{\lambda}^{p-1}}{\omega_{(p-1)(2 r+1)+l} \ldots \omega_{(2 r+1)+l}} u_{p(2 r+1)+l} \\
& \left.+\sum_{p \geq 1} \frac{\omega_{l-p(2 r+1)} \ldots \omega_{l-(2 r+1)}}{\bar{\lambda}^{p}} u_{-p(2 r+1)+l}\right)(\lambda-A)^{*} e_{l}=0
\end{aligned}
$$

for every $r<|\lambda|<R$ and every $l=-r, \ldots, r$, from which it follows that

$$
\begin{aligned}
\sum_{l=-r}^{r} \frac{1}{\omega_{l}}\left(u_{(2 r+1)+l}\right. & +\sum_{p \geq 2} \frac{\bar{\lambda}^{p-1}}{\omega_{(p-1)(2 r+1)+l} \ldots \omega_{(2 r+1)+l}} u_{p(2 r+1)+l} \\
& \left.+\sum_{p \geq 1} \frac{\omega_{l-p(2 r+1)} \ldots \omega_{l-(2 r+1)}}{\bar{\lambda}^{p}} u_{-p(2 r+1)+l}\right)=0
\end{aligned}
$$


for every $r<|\lambda|<R$ and every $l=-r, \ldots, r$. Hence $u=0$, and Proposition 2.14 is proved.

Remark 2.15. - The description of the eigenvectors of $S_{A, \omega}$ given in (4) above shows that if the two series

$$
\sum_{p \geq 1}\left(\omega_{p(2 r+1)+l} \ldots \omega_{(2 r+1)+l}\right)^{-2} \text { and } \sum_{p \geq 1}\left(\omega_{l-p(2 r+1)} \ldots \omega_{l-(2 r+1)}\right)^{-2}
$$

are divergent for every $|l| \leq r$, then the operator $S_{A, \omega}$ has no eigenvalue, whatever the choice of $A \in \mathfrak{B}\left(\mathcal{H}_{r}\right)$. This observation will be useful for the proof of Proposition 2.22 below. It is also interesting to observe that the assumptions on $R_{l}$ and $r_{l}$ in Proposition 2.14 do not involve the operator $A$, contrary to what happens in Proposition 2.10. This is coherent with the fact that the eigenvalues of $A$ do not necessarily appear as eigenvalues of $S_{A, \boldsymbol{\omega}}$, while they do appear as eigenvalues of $B_{A, \boldsymbol{\omega}}$.

2.4. Topological weak mixing and topological mixing. - In this subsection, we show that topological weak mixing is a typical property, whereas topological mixing is atypical. In view of the corresponding well-known analogues in ergodic theory due to Halmos and Rohlin (see e.g. [33, pp. 77-80], and [20] for a more general result), this should not be surprising at all.

Proposition 2.16. - For every $M>1$, the class $\operatorname{TWMIX}_{M}(\mathcal{H})$ is a dense $G_{\delta}$ (and hence comeager) subset of $\left(\mathrm{HC}_{M}(\mathcal{H}), \mathrm{SOT}^{*}\right)$.

Proof. - That TWMIX $M(\mathcal{H})$ is $G_{\delta}$ follows from the fact that $\operatorname{HC}_{M}(\mathcal{H} \times \mathcal{H})$ is $G_{\delta}$ in $\mathfrak{B}_{M}(\mathcal{H} \times \mathcal{H})$, and the SOT*-continuity of the map $T \mapsto T \times T$. Since operators in G-MIX $(\mathcal{H})$ are topologically mixing, density follows from Corollary 2.12 .

Proposition 2.17. - For every $M>1$, the class $\operatorname{TMIX}_{M}(\mathcal{H})$ is meager in the space $\left(\mathrm{HC}_{M}(\mathcal{H})\right.$, SOT$\left.^{*}\right)$.

The proof of Proposition 2.17 relies on Lemmas 2.30 and 2.31, which belong to the forthcoming Section [2.6. We nonetheless prefer to present things in this order, because the purposes of Lemmas 2.30 and 2.31 will appear more clearly in our proof of the typicality of operators without non-trivial invariant measures.

Proof. - By Fact 2.4, it is enough to show that the class $\operatorname{TMIX}_{M}(\mathcal{H})$ is meager in $\left(\mathfrak{B}_{M}(\mathcal{H})\right.$, SOT $\left.^{*}\right)$. Let $B$ be a non-trivial closed ball in $\mathcal{H}$. We certainly have

$$
\operatorname{TMIX}_{M}(\mathcal{H}) \subseteq \bigcup_{N \geq 1} \mathfrak{F}_{N}
$$

where

$$
\mathfrak{F}_{N}:=\bigcap_{n \geq N}\left\{T \in \mathfrak{B}_{M}(\mathcal{H}) ; T^{n}(B) \cap B \neq \emptyset\right\} \quad \text { for every } N \geq 1 .
$$

Each set $\mathfrak{F}_{N}$ is closed in $\left(\mathfrak{B}_{M}(\mathcal{H})\right.$, SOT$\left.^{*}\right)$. Indeed, we may write

$$
T \in \mathfrak{F}_{N} \Longleftrightarrow \forall n \geq N \exists x \in B: T^{n} x \in B .
$$

Since $B$ is weakly closed in $\mathcal{H}$, the condition " $T{ }^{n} x \in B$ " defines a closed subset of $\left(\mathfrak{B}_{M}(\mathcal{H}), \mathrm{SOT}^{*}\right) \times(B, w)$ by Facts 2.1 and 2.5 , and since $B$ is weakly compact, this shows that $\mathfrak{F}_{N}$ is closed in $\left(\mathfrak{B}_{M}(\mathcal{H}), \mathrm{SOT}^{*}\right)$. To conclude the proof, it is enough to show 
that for some suitable choice of the ball $B$, the closed sets $\mathfrak{F}_{N}$ have empty interior in $\left(\mathfrak{B}_{M}(\mathcal{H}), \mathrm{SOT}^{*}\right)$; or, equivalently, that the open sets

$$
\mathfrak{O}_{N}:=\left\{T \in \mathfrak{B}_{M}(\mathcal{H}) ; \exists n \geq N: T^{n}(B) \cap B=\emptyset\right\}
$$

are dense in $\left(\mathfrak{B}_{M}(\mathcal{H})\right.$, SOT $\left.^{*}\right)$. We choose for $B$ the ball $\bar{B}(e, 1 / 2)$, where $e \in \mathcal{H}$ satisfies $\|e\|=1$. Then $\mathfrak{O}_{N}$ contains the set $\mathfrak{O}_{N+1, B}$ of Lemma 2.30 below, so it is dense in $\left(\mathfrak{B}_{M}(\mathcal{H})\right.$, SOT $\left.^{*}\right)$ by Lemma 2.31 .

Remark 2.18. - The same proofs would show that topological weak mixing is typical and topological mixing is atypical for operators on $\ell_{p}$ spaces. It would be interesting to know if this is still true on every Banach space with separable dual. In this respect, it is worth mentioning that there exist on any separable Banach space hypercyclic operators which are not topologically mixing $([\mathbf{3 0}])$.

2.4.1. Some illustrations. - In this subsection, we present some consequences of Propositions 2.16 and 2.17.

First, we have the following amusing fact: a typical operator $T \in \mathrm{HC}_{M}(\mathcal{H}), M>1$, satisfies the Hypercyclicity Criterion but not Kitai's criterion.

In the same spirit, it follows from Proposition 2.16 and the Baire Category Theorem that a typical $T \in \mathfrak{B}_{M}(\mathcal{H})$ is such that $T$ and $T^{*}$ are both topologically weakly mixing; but no operator with this property can be topologically mixing since otherwise $T \times T^{*}$ would be hypercyclic on $\mathcal{H} \times \mathcal{H}$, which can never happen.

Here is now a less immediate consequence of the comeagerness of $\operatorname{TWMIX}_{M}(\mathcal{H})$, which is a partial strengthening of the main theorem of [53. This result could also be easily deduced from [26. Th. 4.1], the proof of which is, however, quite different.

Proposition 2.19. - Let $Z$ be a linear subspace of $\mathcal{H}$ with countable algebraic dimension. For any $M>1$, the set of all $T \in \mathfrak{B}_{M}(\mathcal{H})$ such that every vector $z \in Z \backslash\{0\}$ is hypercyclic for $T$ is comeager in $\left(\mathfrak{B}_{M}(\mathcal{H})\right.$, $\left.\mathrm{SOT}^{*}\right)$.

Proof. - Let us first recall that any topologically weakly mixing operator $T$ on $\mathcal{H}$ is in fact totally hypercyclic, which means that for any $N \geq 1$, the $N$-fold product operator $T_{N}:=T \times \cdots \times T$ is hypercyclic on $\mathcal{H}^{N}:=\mathcal{H} \times \cdots \times \mathcal{H}$. This is a classical result, which has nothing to do with linearity; see e.g [24], or [7, Th. 4.6].

For any finite sequence $\mathbf{f}=\left(f_{1}, \ldots, f_{N}\right)$ of vectors of $\mathcal{H}$, we will denote by $\operatorname{Gram}(\mathbf{f})$ the associated Gram matrix,

$$
\operatorname{Gram}(\mathbf{f}):=\left(\left\langle f_{i}, f_{j}\right\rangle\right)_{1 \leq i, j \leq N}
$$

and we define $\mathcal{H}_{\mathbf{f}}:=\left\{\mathbf{x}=\left(x_{1}, \ldots, x_{N}\right) \in \mathcal{H}^{N} ; \operatorname{Gram}(\mathbf{x})=\operatorname{Gram}(\mathbf{f})\right\}$. Note that $\mathcal{H}_{\mathbf{f}}$ is a closed subset of $\mathcal{H}^{N}$, and hence a Polish space. The key point in the proof of Proposition 2.19 is the following fact.

Fact 2.20. - Let $\mathbf{f}=\left(f_{1}, \ldots, f_{N}\right)$ be a finite sequence of linearly independent vectors in $\mathcal{H}$. For any operator $T \in \operatorname{TWMIX}(\mathcal{H})$, the set $\mathcal{H}_{\mathbf{f}} \cap \mathrm{HC}\left(T_{N}\right)$ is dense in $\mathcal{H}_{\mathbf{f}}$.

Proof of Fact 2.20 - Let $\left(u_{1}, \ldots, u_{N}\right) \in \mathcal{H}_{\mathbf{f}}$ be arbitrary. We are looking for some $N$ tuple $\left(x_{1}, \ldots, x_{N}\right) \in \mathcal{H}_{\mathbf{f}}$ such that $\left(x_{1}, \ldots, x_{N}\right)$ lies in $\operatorname{HC}\left(T_{N}\right)$ and $\left(x_{1}, \ldots, x_{N}\right)$ is very close to $\left(u_{1}, \ldots, u_{N}\right)$. Since $\operatorname{HC}\left(T_{N}\right)$ is dense in $\mathcal{H}^{N}$, one can first choose $\left(z_{1}, \ldots, z_{N}\right) \in$ $\mathrm{HC}\left(T_{N}\right)$ very close to $\left(u_{1}, \ldots, u_{N}\right)$. 
Note that the $N$-tuple $\mathbf{u}=\left(u_{1}, \ldots, u_{N}\right)$ consists of linearly independent vectors since the Gram matrix $\operatorname{Gram}(\mathbf{u})=\operatorname{Gram}(\mathbf{f})$ is invertible; and $\left(z_{1}, \ldots, z_{N}\right)$ is linearly independent as well since $\left(z_{1}, \ldots, z_{N}\right)$ belongs to $\operatorname{HC}\left(T_{N}\right)$. Let us denote by $\left(\widetilde{u}_{1}, \ldots, \widetilde{u}_{N}\right)$ and $\left(\widetilde{z}_{1}, \ldots, \widetilde{z}_{N}\right)$ the sequences obtained by applying the Gram-Schmidt orthonormalization process to $\left(u_{1}, \ldots, u_{N}\right)$ and $\left(z_{1}, \ldots, z_{N}\right)$ respectively. Then $\left(\widetilde{u}_{1}, \ldots, \widetilde{u}_{N}\right)$ and $\left(\widetilde{z}_{1}, \ldots, \widetilde{z}_{N}\right)$ are very close to each other, provided $\left(u_{1}, \ldots, u_{N}\right)$ and $\left(z_{1}, \ldots, z_{N}\right)$ are sufficiently close. Now, define $\left(x_{1}, \ldots, x_{N}\right) \in \mathcal{H}^{N}$ as follows:

$$
x_{i}:=\sum_{l=1}^{N}\left\langle u_{i}, \widetilde{u}_{l}\right\rangle \widetilde{z}_{l} \quad \text { for } i=1, \ldots, N .
$$

We have by definition

$$
\left\langle x_{i}, x_{j}\right\rangle=\sum_{l=1}^{N}\left\langle u_{i}, \widetilde{u}_{l}\right\rangle \overline{\left\langle u_{j}, \widetilde{u}_{l}\right\rangle}=\left\langle u_{i}, u_{j}\right\rangle=\left\langle f_{i}, f_{j}\right\rangle \quad \text { for } i, j=1, \ldots, N,
$$

so that $\left(x_{1}, \ldots, x_{N}\right)$ belongs to $\mathcal{H}_{\mathbf{f}}$. Moreover, each vector $x_{i}, 1 \leq i \leq N$, is very close to $\sum_{l=1}^{N}\left\langle u_{i}, \widetilde{u}_{l}\right\rangle \widetilde{u}_{l}=u_{i}$, so that $\left(x_{1}, \ldots, x_{N}\right)$ is very close to $\left(u_{1}, \ldots, u_{N}\right)$. It remains to show that $\left(x_{1}, \ldots, x_{N}\right)$ belongs to $\mathrm{HC}\left(T_{N}\right)$.

Since the vectors $x_{i}$ are linearly independent (because $\left(x_{1}, \ldots, x_{N}\right)$ belongs to $\left.\mathcal{H}_{\mathbf{f}}\right)$ and belong to $\operatorname{span}\left[\widetilde{z}_{1}, \ldots, \widetilde{z}_{N}\right]=\operatorname{span}\left[z_{1}, \ldots, z_{N}\right]$, they form a basis of $\operatorname{span}\left[z_{1}, \ldots, z_{N}\right]$. So we may write each vector $x_{i}$ as

$$
x_{i}=\sum_{j=1}^{N} c_{i, j} z_{j},
$$

where the matrix $\left(c_{i, j}\right)_{1 \leq i, j \leq N}$ is invertible. Now, let $V_{1}, \ldots, V_{N}$ be non-empty open sets in $\mathcal{H}$, and define

$$
\mathcal{V}:=\left\{\left(y_{1}, \ldots, y_{N}\right) \in \mathcal{H}^{N} ; \sum_{j=1}^{N} c_{i, j} y_{j} \in V_{i} \quad \text { for } i=1, \ldots, N\right\} .
$$

Since the matrix $\left(c_{i j}\right)_{1 \leq i, j \leq N}$ is invertible, this is a non-empty open subset of $\mathcal{H}^{N}$. As $\left(z_{1}, \ldots, z_{N}\right) \in \operatorname{HC}\left(T_{N}\right)$, one can find an integer $n$ such that $\left(T^{n} z_{1}, \ldots, T^{n} z_{N}\right) \in \mathcal{V}$, which means that $\left(T^{n} x_{1}, \ldots, T^{n} x_{N}\right) \in V_{1} \times \cdots \times V_{N}$ by the definition of $\mathcal{V}$. This shows that $\left(x_{1}, \ldots, x_{N}\right)$ belongs to $\mathrm{HC}\left(T_{N}\right)$.

From Fact 2.20, we now deduce:

Fact 2.21. - Let $M>1$. For any finite family $\mathbf{f}=\left(f_{1}, \ldots, f_{N}\right)$ of linearly independent vectors in $\mathcal{H}$, the set of all $T \in \mathfrak{B}_{M}(\mathcal{H})$ such that $\left(f_{1}, \ldots, f_{N}\right)$ belongs to $\operatorname{HC}\left(T_{N}\right)$ is comeager in $\left(\mathfrak{B}_{M}(\mathcal{H}), \mathrm{SOT}^{*}\right)$.

Proof of Fact 2.21. - Let us consider the set

$$
\mathcal{G}:=\left\{\left(x_{1}, \ldots, x_{N}, T\right) \in \mathcal{H}_{\mathbf{f}} \times \mathfrak{B}_{M}(\mathcal{H}) ;\left(x_{1}, \ldots, x_{N}\right) \in \mathrm{HC}\left(T_{N}\right)\right\} .
$$

This is a $G_{\delta}$ subset of $\mathcal{H}_{\mathbf{f}} \times\left(\mathfrak{B}_{M}(\mathcal{H}), \mathrm{SOT}^{*}\right)$. Moreover, Fact 2.20 asserts that for any $T \in \operatorname{TWMIX}_{M}(\mathcal{H})$, the $T$-section of $\mathcal{G}$ is dense in $\mathcal{H}_{\mathbf{f}}$, and hence comeager in $\mathcal{H}_{\mathbf{f}}$ since this is a $G_{\delta}$ set. Since $\operatorname{TWMIX}_{M}(\mathcal{H})$ is comeager in $\left(\mathfrak{B}_{M}(\mathcal{H}), \mathrm{SOT}^{*}\right)$, it follows, by the 
Kuratowski-Ulam Theorem (see for instance [37, Section 8.K]), that there exists at least one (in fact, comeager many) $\mathbf{x}=\left(x_{1}, \ldots, x_{N}\right) \in \mathcal{H}_{\mathbf{f}}$ such that the set

$$
\mathfrak{G}_{\mathbf{x}}:=\left\{T \in \mathfrak{B}_{M}(\mathcal{H}) ;\left(x_{1}, \ldots, x_{N}\right) \in \operatorname{HC}\left(T_{N}\right)\right\}
$$

is comeager in $\left(\mathfrak{B}_{M}(\mathcal{H}), \mathrm{SOT}^{*}\right)$.

Now, since $\left(x_{1}, \ldots, x_{N}\right)$ belongs to $\mathcal{H}_{\mathbf{f}}$, i.e. $\left\langle x_{i}, x_{j}\right\rangle=\left\langle f_{i}, f_{j}\right\rangle$ for every $i, j=1 \ldots, N$, one can find a unitary operator $U: \mathcal{H} \rightarrow \mathcal{H}$ such that $U f_{i}=x_{i}$ for every $i=1, \ldots, N$. Then the map $T \mapsto U^{-1} T U$ maps $\mathfrak{B}_{M}(\mathcal{H})$ bijectively onto itself because $U$ is unitary, and is a homeomorphism with respect to the topology SOT* Therefore, the set $\mathfrak{G}_{\mathrm{f}}:=U^{-1} \mathfrak{G}_{\mathbf{x}} U$ is comeager in $\left(\mathfrak{B}_{M}(\mathcal{H})\right.$, SOT* $)$. Since by definition $\left(f_{1}, \ldots, f_{N}\right)$ belongs to $\mathrm{HC}\left(T_{N}\right)$ if $T$ belongs to $\mathfrak{G}_{\mathfrak{f}}$, this concludes the proof of Fact 2.21

We are now ready to conclude the proof of Proposition 2.19, Let $\left(f_{i}\right)_{i \geq 1}$ be an algebraic basis of $Z$. By Fact 2.21, the set

$$
\mathfrak{G}:=\left\{T \in \mathfrak{B}_{M}(\mathcal{H}) ; \forall N \geq 1:\left(f_{1}, \ldots, f_{N}\right) \in \operatorname{HC}\left(T_{N}\right)\right\}
$$

is comeager in $\left(\mathfrak{B}_{M}(\mathcal{H}), \mathrm{SOT}^{*}\right)$. So it is enough to show that that if $T$ belongs to $\mathfrak{G}$, every non-zero vector $z \in Z$ is a hypercyclic vector for $T$. Let $V$ be a non-empty open set in $\mathcal{H}$. Write $z$ as $z=\sum_{i=1}^{N} z_{i} f_{i}$, and consider the set

$$
\mathcal{V}_{z}:=\left\{\left(x_{1}, \ldots, x_{N}\right) \in \mathcal{H}^{N} ; \sum_{i=1}^{N} z_{i} x_{i} \in V\right\} .
$$

Since $z \neq 0$, this is a non-empty open set in $\mathcal{H}^{N}$. As $T$ belongs to $\mathfrak{G}$, it follows that there exists $n \geq 1$ such that $\left(T^{n} f_{1}, \ldots, T^{n} f_{N}\right)$ belongs to $\mathcal{V}_{z}$, which means exactly that $T^{n} z$ belongs to $V$. Hence $z$ is indeed a hypercyclic vector for $T$.

2.5. Hypercyclic operators without eigenvalues. - The following result shows that operators without eigenvalues are typical.

Proposition 2.22. - For any $M>1$, the class $\operatorname{NEV}_{M}(\mathcal{H})$ is a dense $G_{\delta}$ subset of $\left(\mathfrak{B}_{M}(\mathcal{H})\right.$, SOT $\left.^{*}\right)$.

From this and Fact 2.4, we obtain

Corollary 2.23. - For any $M>1$, a typical operator $T \in\left(\mathrm{HC}_{M}(\mathcal{H})\right.$, $\left.\mathrm{SOT}^{*}\right)$ has no eigenvalues.

As a matter of fact, Proposition 2.22 is already proved in 21, where typical properties of contraction operators are studied for various topologies (see also [19]). However, since the proof is not that complicated, and in order to keep the paper as self-contained as possible, we outline it below.

Proof of Proposition 2.22. - We divide the proof into two steps. In what follows, we fix $M>0$.

Fact 2.24. - The set $\operatorname{NEV}_{M}(\mathcal{H})$ is a $G_{\delta}$ subset of $\left(\mathfrak{B}_{M}(\mathcal{H})\right.$, $\left.\mathrm{SOT}^{*}\right)$. 
Proof of Fact 2.24 - To any closed ball $B \subseteq \mathcal{H}$, we associate the following subset of $\mathfrak{B}_{M}(\mathcal{H})$ :

$$
\mathfrak{M}_{B}=\left\{T \in \mathfrak{B}_{M}(\mathcal{H}) ; \exists \lambda \in \mathbb{C}, \exists x \in B \text { with } T x=\lambda x\right\} .
$$

Let us show that this set $\mathfrak{M}_{B}$ is $F_{\sigma}$ in $\left(\mathfrak{B}_{M}(\mathcal{H}), \mathrm{SOT}^{*}\right)$. To this aim, we endow $B$ with the weak topology, and introduce the set

$$
\mathcal{F}_{B}=\left\{(T, \lambda, x) \in \mathfrak{B}_{M}(\mathcal{H}) \times \mathbb{C} \times B ; T x=\lambda x\right\} .
$$

Then $\mathfrak{M}_{B}$ is the projection of $\mathcal{F}_{B}$ on the first coordinate. Moreover, the set $\mathcal{F}_{B}$ is closed in $\left(\mathfrak{B}_{M}(\mathcal{H}), \mathrm{SOT}^{*}\right) \times \mathbb{C} \times(B, w)$ by Fact 2.5. Since the space $\mathbb{C} \times(B, w)$ is $K_{\sigma}$ (because $(B, w)$ is compact), it follows that $\mathfrak{M}_{B}$ is $F_{\sigma}$.

Let now $\left(B_{q}\right)_{q \geq 1}$ be a sequence of closed balls of $\mathcal{H}$ not containing the point 0 , such that $\bigcup_{q \geq 1} B_{q}=H \backslash\{0\}$. Then $\mathfrak{B}_{M}(\mathcal{H}) \backslash \operatorname{NEV}_{M}(\mathcal{H})=\bigcup_{q \geq 1} \mathfrak{M}_{B_{q}}$ is an $F_{\sigma}$ set, so that $\operatorname{NEV}_{M}(\mathcal{H})$ is a $G_{\delta}$ set in $\left(\mathfrak{B}_{M}(\mathcal{H})\right.$, SOT $\left.^{*}\right)$.

Fact 2.25. - The set $\operatorname{NEV}_{M}(\mathcal{H})$ is dense in $\left(\mathfrak{B}_{M}(\mathcal{H})\right.$, SOT$\left.^{*}\right)$.

Proof of Fact 2.25, - Let $\left(f_{k}\right)_{k \in \mathbb{Z}}$ be an orthonormal basis of $\mathcal{H}$. For each $r \geq 0$, we set $\mathcal{H}_{r}:=\operatorname{span}\left[f_{k}, ;|k| \leq r\right]$ and we denote by $P_{r}$ the orthogonal projection of $\mathcal{H}$ onto $\mathcal{H}_{r}$. By Lemma 2.6 and Remark 2.7, it suffices to show that for every $r \geq 0$, every $A \in \mathfrak{B}\left(\mathcal{H}_{r}\right)$, with $\|A\|<M$, and every $\varepsilon>0$, there exists an operator $T \in \operatorname{NEV}_{M}(\mathcal{H})$ such that

$$
\left\|(T-A) f_{k}\right\|<\varepsilon \quad \text { and } \quad\left\|(T-A)^{*} f_{k}\right\|<\varepsilon \quad \text { for every } k=-r, \ldots, r .
$$

Let $\boldsymbol{\omega}=\left(\omega_{k}\right)_{k \in \mathbb{Z}}$ be a bilateral weight sequence with $0<\omega_{k} \leq M$ for every $k \in \mathbb{Z}$ and $\omega_{k}=\delta$ for every index $k$ with $|k+r| \leq 2 r+1$. As in the proof of Corollary 2.12, one easily checks that if $\delta>0$ is sufficiently small, the bilateral weighted shift operator $S_{A, \boldsymbol{\omega}}$ (defined with respect to the basis $\left(f_{k}\right)_{k \in \mathbb{Z}}$ ) satisfies

$$
\left\|\left(S_{A, \omega}-A\right) f_{k}\right\|<\varepsilon \quad \text { and } \quad\left\|\left(S_{A, \omega}-A\right)^{*} f_{k}\right\|<\varepsilon \quad \text { for every } k=-r, \ldots, r .
$$

Moreover, if the weight sequence $\boldsymbol{\omega}$ is chosen in such a way that the series

$$
\sum_{p \geq 1}\left(\omega_{p(2 r+1)+l} \ldots \omega_{(2 r+1)+l}\right)^{-2} \text { and } \sum_{p \geq 1}\left(\omega_{l-p(2 r+1)} \cdots \omega_{l-(2 r+1)}\right)^{-2}
$$

are divergent for every $|l| \leq r$, then $S_{A, \boldsymbol{\omega}}$ has no eigenvalue by Remark 2.15. So $T:=S_{A, \boldsymbol{\omega}}$ satisfies the required assumptions for a suitable choice of the weight $\omega$.

The two facts above complete the proof of Proposition 2.22,

Remark 2.26. - The fact that we are using the topology SOT* is crucial in the proof of Fact 2.24 in order to obtain that the sets $\mathcal{F}_{B}$ above are closed in $\left(\mathfrak{B}_{M}(\mathcal{H})\right.$, SOT$\left.^{*}\right) \times \mathbb{C} \times$ $(B, w)$. The situation turns out to be completely different if one considers the topology SOT instead of SOT*. Indeed, it is proved in 21 that an SOT-typical $T \in \mathfrak{B}_{1}(\mathcal{H})$ has the property that every $\lambda \in \mathbb{C}$ with $|\lambda|<1$ is an eigenvalue of $T$. More precisely, a typical $T \in \mathfrak{B}_{1}(\mathcal{H})$ is unitarily equivalent to the infinite-dimensional backward unilateral shift operator. So a typical $T \in\left(\mathfrak{B}_{M}(\mathcal{H})\right.$, SOT $)$ has the whole disk $D(0, M)$ within the set of its eigenvalues.

Remark 2.27. - The proof of Corollary 2.23 is a good example of the usefulness of Fact 2.4 for simplifying arguments of this kind: although it is easy to construct the weight sequence $\boldsymbol{\omega}$ above in such a way that the operator $S_{A, \boldsymbol{\omega}}$ is SOT*-close to $A$ and has no eigenvalue, it is technically much less obvious to ensure that $S_{A, \boldsymbol{\omega}}$ is additionally 
hypercyclic. In other words, it would be less easy to prove Corollary 2.23 directly, without using Fact 2.4.

One of the main consequences of Corollary 2.23] is the following result concerning chaotic operators.

Corollary 2.28. - For every $M>1, \mathrm{CH}_{M}(\mathcal{H})$ is meager in $\left(\mathrm{HC}_{M}(\mathcal{H}), \mathrm{SOT}^{*}\right)$. In other words, a typical hypercyclic operator on $\mathcal{H}$ is not chaotic.

This is indeed obvious since chaotic operators have plenty of eigenvalues. Note that we are using here the fact that $\mathcal{H}$ is a complex Hilbert space, so that periodic points are linear combinations of eigenvectors whose associated eigenvalues are roots of unity. Nonetheless, Corollary 2.28 holds true on real Hilbert spaces as well; see Remark 2.34 below.

2.6. Hypercyclic operators without invariant measures. - It follows easily from Proposition 2.22 that for any $M>1$, the operators in $\mathrm{HC}_{M}(\mathcal{H})$ admitting a non-trivial invariant measure with a second-order moment form a meager class in $\left(\mathrm{HC}_{M}(\mathcal{H}), \mathrm{SOT}^{*}\right)$. Indeed, if $T \in \mathrm{HC}_{M}(\mathcal{H})$ admits an invariant measure $\mu \neq \delta_{0}$ such that $\int_{H}\|x\|^{2} d \mu(x)<\infty$, then the Gaussian measure $m$ whose covariance operator is given by the formula

$$
\langle R x, y\rangle=\int_{H}\langle x, z\rangle \overline{\langle y, z\rangle} d \mu(z), \quad x, y \in H
$$

is $T$-invariant. Its support is the closed linear span of the support of $\mu$, and hence is non-trivial. This closed subspace is spanned by unimodular eigenvectors of $T$ (see [4] or [7] for details), from which it follows that $T$ does not belong to $\operatorname{NEV}_{M}(\mathcal{H})$.

The main result of this subsection is that the SOT*-typical operator $T \in \mathrm{HC}_{M}(\mathcal{H})$ actually admits no non-trivial invariant measure at all.

Theorem 2.29. - For every $M>1$, the set $\operatorname{HC}_{M}(\mathcal{H}) \backslash \operatorname{INV}(\mathcal{H})$ is comeager in the space $\left(\mathrm{HC}_{M}(\mathcal{H}), \mathrm{SOT}^{*}\right)$.

The proof of Theorem 2.29 relies on the next two lemmas.

Lemma 2.30. - Let $B$ be a closed ball of $\mathcal{H}$ not containing the point 0 . For any integer $n \geq 1$, the set

$$
\begin{aligned}
& \mathfrak{O}_{n, B}=\left\{T \in \mathfrak{B}_{M}(\mathcal{H}) ; \text { there exist } n \text { distinct integers } p_{1}, \ldots, p_{n}\right. \text { such that } \\
& \left.\qquad T^{p_{i}}(B) \cap T^{p_{j}}(B)=\emptyset \text { for every } i \neq j, 1 \leq i, j \leq n\right\}
\end{aligned}
$$

is open in $\left(\mathfrak{B}_{M}(\mathcal{H}), \mathrm{SOT}^{*}\right)$. Consequently, the set

$\mathfrak{G}_{B}=\left\{T \in \mathfrak{B}_{M}(\mathcal{H}) ;\right.$ for every $n \geq 1$, there exist $n$ iterates of $B$

under the action of $T$ which are pairwise disjoint $\}$

is $G_{\delta}$ in $\left(\mathfrak{B}_{M}(\mathcal{H})\right.$, SOT $\left.^{*}\right)$.

Proof of Lemma 2.30 - The second part of the lemma follows immediately from the first, since $\mathfrak{G}_{B}=\bigcap_{n \geq 1} \mathfrak{O}_{n, B}$. To derive the first part, it is enough to show that if we fix $p, q \geq 1$, then the set

$$
\mathfrak{O}:=\left\{T \in \mathfrak{B}_{M}(\mathcal{H}) ; T^{p}(B) \cap T^{q}(B)=\emptyset\right\}
$$

is $\mathrm{SOT}^{*}$-open in $\mathfrak{B}_{M}(\mathcal{H})$. If $T \in \mathfrak{B}_{M}(\mathcal{H})$, we may write

$$
T \in \mathfrak{O} \Longleftrightarrow \forall x, y \in B: T^{p} x \neq T^{q} y .
$$


Since the map $(T, u) \mapsto T^{n} u$ is continuous on $\left(\mathfrak{B}_{M}(\mathcal{H})\right.$, $\left.\mathrm{SOT}^{*}\right) \times(B, w)$ for any $n \geq 1$ by Facts 2.1 and 2.5, the condition " $T^{p} x \neq T^{q} y$ " defines an open subset of $\left(\mathfrak{B}_{M}(\mathcal{H}), \mathrm{SOT}^{*}\right) \times$ $(B, w) \times(B, w)$. Since $B$ is weakly compact, it follows that $\mathfrak{O}$ is indeed SOT*-open in $\mathfrak{B}_{M}(\mathcal{H})$, its complement being the projection of a closed subset of $\mathfrak{B}_{M}(\mathcal{H}) \times B \times B$ along the compact factor $B \times B$.

Lemma 2.31. - Let $e \in \mathcal{H}$ with $\|e\|=1$, and let $0<\rho<1$. Denote by $B$ the closed ball $\bar{B}(e, \rho)$. Let also $M>1$. For any $n \geq 1$, the open set $\mathfrak{O}_{n, B}$ is dense in $\left(\mathfrak{B}_{M}(\mathcal{H}), \mathrm{SOT}^{*}\right)$.

Proof of Lemma 2.31 - Let us fix an orthonormal basis $\left(e_{k}\right)_{k \geq 1}$ of $\mathcal{H}$ with $e_{1}=e$. Our aim being to apply Lemma 2.6, we fix $r \geq 1$, an operator $A \in \mathfrak{B}\left(\mathcal{H}_{r}\right)$ such that $\|A\|<M$, and $\varepsilon>0$. We are looking for an operator $T \in \mathfrak{O}_{n, B}$ such that

$$
\left\|(T-A) e_{k}\right\|<\varepsilon \text { and }\left\|(T-A)^{*} e_{k}\right\|<\varepsilon \quad \text { for } k=1, \ldots, r .
$$

We will define a sequence $\left(C_{N}\right)_{N>2 r}$ of operators, with $C_{N} \in \mathfrak{B}\left(\mathcal{H}_{N}\right)$ for every $N>2 r$, and show that if $N$ is sufficiently large, the operator $P_{N}^{*} C_{N} P_{N} \in \mathfrak{B}(\mathcal{H})$ belongs to $\mathfrak{O}_{n, B}$ and satisfies the above estimates. Here $P_{N}$ denotes as usual the canonical projection of $\mathcal{H}$ onto $\mathcal{H}_{N}$.

Let $\delta>0$ and $\gamma>1$, to be fixed later on in the proof. For each $N>2 r$, consider the operator $C_{N} \in \mathfrak{B}\left(\mathcal{H}_{N}\right)$ defined in the following way:

$$
C_{N} e_{k}= \begin{cases}A e_{k}+\delta e_{k+r} & \text { for every } 1 \leq k \leq r \\ \gamma e_{k+r} & \text { for every } r<k \leq N-r \\ 0 & \text { for every } N-r<k \leq N .\end{cases}
$$

Thus, in matrix form,

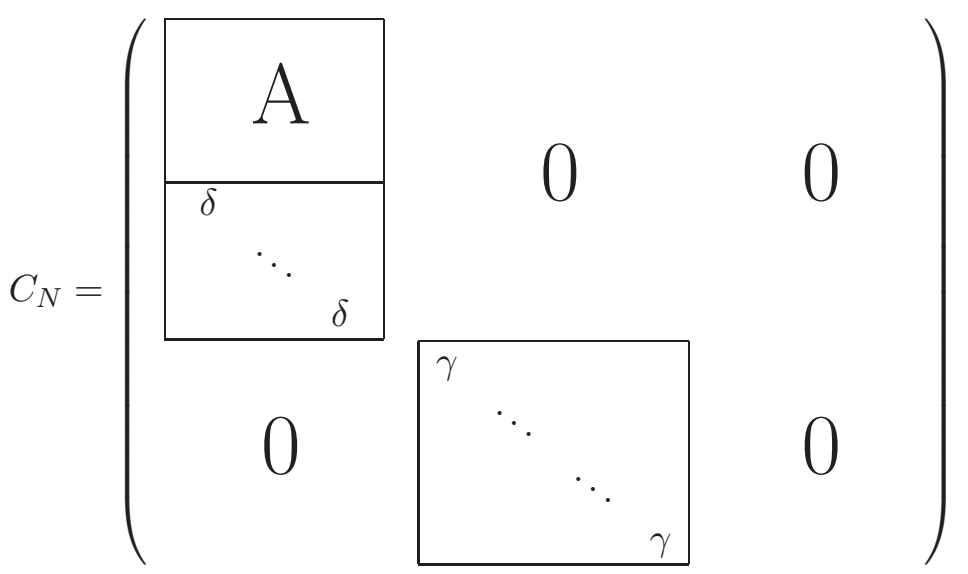

We note that if $\|A\|<\gamma<M$ and if $\delta$ is sufficiently small, then $\left\|C_{N}\right\|=\gamma<M$. Moreover, if $\delta$ is sufficiently small, then

$$
\left\|\left(C_{N}-A\right) e_{k}\right\|<\varepsilon \quad \text { and } \quad\left\|\left(C_{N}-A\right)^{*} e_{k}\right\|<\varepsilon \quad \text { for every } k=1, \ldots, r
$$

whatever the choice of the integer $N>2 r$. The key of the proof lies in the following simple computation.

Fact 2.32. - For every $N>2 r$, every $p \geq 1$ such that $p r+1 \leq N$, and every $x \in \mathcal{H}$,

$$
\left\langle C_{N}^{p} x, e_{p r+1}\right\rangle=\gamma^{p-1} \delta\left\langle x, e_{1}\right\rangle .
$$


Proof of Fact 2.32, Clearly $\left\langle C_{N} x, e_{r+1}\right\rangle=\delta\left\langle x, e_{1}\right\rangle$; so we may write $C_{N} x$ as

Hence

$$
C_{N} x=\delta\left\langle x, e_{1}\right\rangle e_{r+1}+\sum_{\substack{k=1 \\ k \neq r+1}}^{N}\left\langle C_{N} x, e_{k}\right\rangle e_{k} .
$$

$$
C_{N}^{p-1} x=\delta \gamma^{p-1}\left\langle x, e_{1}\right\rangle e_{p r+1}+\sum_{\substack{k=1 \\ k \neq r+1}}^{N}\left\langle C_{N} x, e_{k}\right\rangle C_{N}^{p-1} e_{k}
$$

Now $C_{N}^{p-1} e_{k}$ belongs to the closed linear span of the vectors $e_{j}, 1 \leq j \leq N, j \neq p r+1$, for every $1 \leq k \leq N$ with $k \neq r+1$. Indeed,

- if $1 \leq k \leq r$, a straightforward induction shows that $C_{N}^{p-1} e_{k} \in \operatorname{span}\left[e_{j} ; 1 \leq j \leq p r\right]$;

- if $r+1<k \leq N-(p-1) r$, then $C_{N}^{p-1} e_{k}=\gamma^{p-1} e_{k+(p-1) r}$;

- if $N-(p-1) r<k \leq N$, we have $C_{N}^{p-1} e_{k}=0$.

Thus $\left\langle C_{N}^{p-1} x, e_{p r+1}\right\rangle=\delta \gamma^{p-1}\left\langle x, e_{1}\right\rangle$, which is the claim of Fact 2.32,

From Fact 2.32, it is not hard to deduce

Fact 2.33. - Let $1 \leq p, q \leq N$ be such that $p r+1 \leq N$. If $\gamma^{p-q-1} \delta>\frac{1+\rho}{1-\rho}$, then $C_{N}^{p}\left(P_{N} B\right) \cap C_{N}^{q}\left(P_{N} B\right)=\emptyset$.

Proof of Fact 2.33 - If $x, y \in P_{N} B$, then by Fact 2.32

$$
\left\|C_{N}^{p} y-C_{N}^{q} x\right\| \geq\left|\left\langle C_{N}^{p} x-C_{N}^{q} y, e_{p r+1}\right\rangle\right| \geq \gamma^{p-1} \delta\left|\left\langle x, e_{1}\right\rangle\right|-\left\|C_{N}^{q}\right\|\|y\| .
$$

Moreover, since $x$ and $y$ belong to $P_{N} B \subseteq B$, we have $\left|\left\langle x, e_{1}\right\rangle\right| \geq 1-\rho$ and $\|y\| \leq 1+\rho$. Hence

$$
\left\|C_{N}^{p} y-C_{N}^{q} x\right\| \geq \gamma^{p-1} \delta(1-\rho)-\gamma^{q}(1+\rho) .
$$

Thus $C_{N}^{q}\left(P_{N} B\right) \cap C_{N}^{p}\left(P_{N}(B)\right)$ is empty as soon as $\gamma^{p-1} \delta(1-\rho)-\gamma^{q}(1+\rho)>0$, which proves our claim.

We now choose the various parameters in this construction in the following order: first we choose $\gamma$ such that $\max (1,\|A\|)<\gamma<M$. Then we choose $\delta>0$ so small that (5) holds true for every $N \geq 1$. Lastly, we choose $N>2 r$ so large that there exist $n$ distinct integers $1 \leq p_{1}<p_{2}<\cdots<p_{n} \leq N$ with $p_{n} r+1 \leq N$, such that the gaps between two consecutive integers $p_{j}$ are so large that $\gamma^{p_{j}-p_{i}-1} \delta>(1-\rho) /(1+\rho)$ for every $1 \leq i<j \leq n$. The operator $C_{N}$ then satisfies $C_{N}^{p_{j}}\left(P_{N} B\right) \cap C_{N}^{p_{i}}\left(P_{N} B\right)=\emptyset$ for every $1 \leq i<j \leq n$. So the operator $T:=P_{N}^{*} C_{N} P_{N} \in \mathfrak{B}_{M}(\mathcal{H})$ is such that $T^{p_{j}}(B) \cap T^{p_{i}}(B)=\emptyset$ for every $1 \leq i<j \leq n$, that is, $T$ belongs to $\mathfrak{O}_{n, B}$; and by (5) we also have $\left\|(T-A) e_{k}\right\|<\varepsilon$ and $\left\|(T-A)^{*} e_{k}\right\|<\varepsilon$ for every $k=1, \ldots, r$.

Proof of Theorem 2.29, - Combining Lemmas 2.30 and 2.31, we obtain that $\mathfrak{G}_{B}$ is a dense $G_{\delta}$ subset of $\left(\mathfrak{B}_{M}(\mathcal{H}), \mathrm{SOT}^{*}\right)$ for every ball $B=\bar{B}(e, \rho)$, where $\|e\|=1$ and $0<\rho<1$. By Fact 2.4, it follows that $\mathfrak{G}_{B} \cap \mathrm{HC}_{M}(\mathcal{H})$ is a dense $G_{\delta}$ subset of $\left(\mathrm{HC}_{M}(\mathcal{H}), \mathrm{SOT}^{*}\right)$ for each such ball $B$. Let $\left(B_{q}\right)_{q \geq 1}$ be a countable family of such balls with the property that

$$
\bigcup_{q \geq 1} B_{q}=B(0,2) \backslash\{0\} .
$$

Then $\mathfrak{G}:=\left(\bigcap_{q \geq 1} \mathfrak{G}_{B_{q}}\right) \cap \mathrm{HC}_{M}(\mathcal{H})$ is a dense $G_{\delta}$ subset of $\left(\mathrm{HC}_{M}(\mathcal{H}), \mathrm{SOT}^{*}\right)$.

Every element $T$ of $\mathfrak{G}$ enjoys the property that for any $q \geq 1$ and any $n \geq 1$, there exist $n$ distinct iterates $T^{j}\left(B_{q}\right)$ of $B_{q}$ which are pairwise distinct. It follows that if $m$ is 
any invariant (probability) measure for $T$, then $m\left(B_{q}\right)=0$ for every $q \geq 1$ and hence $m(B(0,2) \backslash\{0\})=0$.

Suppose now that $T \in \mathfrak{G}$ admits an invariant measure $m \neq \delta_{0}$. Then one can find a closed ball $B^{\prime}$ not containing 0 such that $m\left(B^{\prime}\right)>0$. Consider for any $R>0$ the measure $m_{R}$ on $\mathcal{H}$ defined by $m_{R}(C)=m(R C)$ for any Borel subset $C$ of $\mathcal{H}$. Each such measure $m_{R}$ is an invariant probability measure on $\mathcal{H}$. Moreover, if $R$ is sufficiently large then $R^{-1} B^{\prime} \subseteq B(0,2) \backslash\{0\}$, so that $m_{R}(B(0,2) \backslash\{0\})>0$, which is a contradiction. We have thus proved that any operator $T \in \mathfrak{G}$ admits no invariant measure except $\delta_{0}$, and hence that $\mathrm{HC}_{M}(\mathcal{H}) \backslash \operatorname{INV}(\mathcal{H})$ is comeager in $\left(\mathrm{HC}_{M}(\mathcal{H})\right.$, $\left.\mathrm{SOT}^{*}\right)$.

Remark 2.34. - The above proof does not use the fact that $\mathcal{H}$ is a complex Hilbert space; so Theorem 2.29 holds true as well for real Hilbert spaces. This shows in particular that in the real setting also, the chaotic operators form a meager subset of $\mathfrak{B}_{M}(\mathcal{H})$; more precisely, a typical operator $T \in \mathfrak{B}_{M}(\mathcal{H})$ has no periodic point except 0 . Indeed, any periodic point $x \neq 0$ for an operator $T$ gives rise to a "canonical" invariant measure supported on the orbit of $x$, namely $m:=\frac{1}{N} \sum_{i=0}^{N-1} \delta_{T^{i} x}$, where $N \geq 1$ is a period of $x$.

Remark 2.35. - Theorem 2.29 implies a weak form of Proposition 2.22, namely that operators without any eigenvalue of modulus 1 are typical. Indeed, if $T$ admits a unimodular eigenvalue and if $x$ is an associated eigenvector, then there is a canonical invariant measure $m \neq \delta_{0}$ supported on $\mathbb{T} \cdot x$, namely the image of the Lebesgue measure on $\mathbb{T}$ under the map $\lambda \mapsto \lambda x$.

Regarding the eigenvalues, one may also note that if $m$ is any non-trivial invariant measure for an operator $T \in \mathfrak{B}(\mathcal{H})$, then $m(\operatorname{ker}(T-\lambda))=0$ for every complex number $\lambda$ such that $|\lambda| \neq 1$. Indeed, if $m \neq \delta_{0}$ is a measure such that $m(\operatorname{ker}(T-\lambda))>0$, one can find a ball $B$ not containing 0 such that $m\left(B_{\lambda}\right) \neq 0$, where $B_{\lambda}=B \cap \operatorname{ker}(T-\lambda)$. Since $|\lambda| \neq 1$, it is easily checked that $B_{\lambda}$ has infinitely many pairwise disjoint iterates under $T$, which is not possible since the measure $m$ is $T$-invariant.

Since $\mathcal{U}$-frequently hypercyclic operators on a Hilbert space always admit an invariant measure with full support by [29, Theorem 2.29 immediately implies:

Corollary 2.36. - For every $M>1, \operatorname{UFHC}_{M}(\mathcal{H})$ is meager in $\left(\mathrm{HC}_{M}(\mathcal{H}), \mathrm{SOT}^{*}\right)$. In other words, a typical hypercyclic operator on $\mathcal{H}$ is not $\mathcal{U}$-frequently hypercyclic.

2.7. Densely distributionally chaotic operators. - In this subsection, our aim is to show that, for any $M>1$, the class $\operatorname{DDCH}_{M}(\mathcal{H})$ of densely distributionally chaotic operators in $\mathfrak{B}_{M}(\mathcal{H})$ is a dense $G_{\delta}$ subset of $\left(\mathfrak{B}_{M}(\mathcal{H})\right.$, SOT $\left.{ }^{*}\right)$, from which it follows that the class $\mathrm{DCH}_{M}(\mathcal{H})$ of distributionally chaotic operators in $\mathfrak{B}_{M}(\mathcal{H})$ is a comeager subset of $\left(\mathfrak{B}_{M}(\mathcal{H})\right.$, SOT $\left.^{*}\right)$.

Proposition 2.37. - For any $M>1$, the set $\mathrm{DDCH}_{M}(\mathcal{H})$ of densely distributionally chaotic operators in $\mathfrak{B}_{M}(\mathcal{H})$ is a dense $G_{\delta}$ subset of $\left(\mathfrak{B}_{M}(\mathcal{H})\right.$, SOT* $)$.

Proof. - Recall that an operator $T \in \mathfrak{B}(\mathcal{H})$ is densely distributionally chaotic if and only if it admits a dense set of distributionally irregular vectors, i.e. of vectors $x \in \mathcal{H}$ for which there exist two sets of integers $A, B \subseteq \mathbb{N}$ with upper density 1 such that $T^{i} x \rightarrow 0$ as $i \rightarrow \infty$ 
along $A$ and $\left\|T^{i} x\right\| \rightarrow \infty$ as $i \rightarrow \infty$ along $B$. Since the set of distributionally irregular vectors for $T$ can be written as

$$
G_{T}=\bigcap_{\varepsilon \in \mathbb{Q}^{+*}} \bigcap_{N \geq 1} G_{T, \varepsilon, N}
$$

where

$$
\begin{aligned}
G_{T, \varepsilon, N}:=\{x \in \mathcal{H} ; \exists m, n \geq N: & \#\left\{1 \leq i \leq m ;\left\|T^{i} x\right\|<\varepsilon\right\} \geq m(1-\varepsilon) \\
& \text { and } \left.\#\left\{1 \leq i \leq n ;\left\|T^{i} x\right\|>1 / \varepsilon\right\} \geq n(1-\varepsilon)\right\},
\end{aligned}
$$

it follows that $G_{T}$ is a dense $G_{\delta}$ subset of $\mathcal{H}$ whenever $T$ is densely distributionally chaotic. Denoting by $\left(V_{p}\right)_{p \geq 1}$ a countable basis of non-empty open subsets of $\mathcal{H}$, we infer from this observation that an operator $T \in \mathfrak{B}_{M}(\mathcal{H})$ belongs to $\operatorname{DDCH}_{M}(\mathcal{H})$ if and only if

$$
\begin{array}{r}
\forall \varepsilon \in \mathbb{Q}^{+*} \forall N \geq 1 \forall p \geq 1 \quad \exists x \in V_{p} \quad \exists n, m \geq N \#\left\{1 \leq i \leq m ;\left\|T^{i} x\right\|<\varepsilon\right\} \geq m(1-\varepsilon) \\
\text { and } \#\left\{1 \leq i \leq n ;\left\|T^{i} x\right\|>1 / \varepsilon\right\} \geq n(1-\varepsilon) .
\end{array}
$$

Using this, we can now prove

Fact 2.38. - The set $\mathrm{DDCH}_{M}(\mathcal{H})$ is a $G_{\delta}$ subset of $\left(\mathfrak{B}_{M}(\mathcal{H})\right.$, SOT $)$.

Proof. - It suffices to show that for every $\varepsilon \in \mathbb{Q}^{+*}, N \geq 1, p \geq 1, m, n \geq N$ and $x \in \mathcal{H}$, the set

$$
\begin{array}{r}
\left\{T \in \mathfrak{B}_{M}(\mathcal{H}) ; \#\left\{1 \leq i \leq m ;\left\|T^{i} x\right\|<\varepsilon\right\} \geq m(1-\varepsilon)\right. \\
\text { and } \left.\#\left\{1 \leq i \leq n ;\left\|T^{i} x\right\|>1 / \varepsilon\right\} \geq n(1-\varepsilon)\right\}
\end{array}
$$

is SOT-open. So let $T_{0} \in \mathfrak{B}_{M}(\mathcal{H})$ belong to this set, and let $r \geq m(1-\varepsilon)$ be an integer such that there exist $r$ indices $1 \leq i_{1}<\cdots<i_{r} \leq m$ such that $\left\|T^{i_{j}} x\right\|<\varepsilon$ for every $1 \leq j \leq r$. If $T \in \mathfrak{B}_{M}(\mathcal{H})$ is sufficiently close to $T_{0}$ for the SOT-topology, we still have $\left\|T^{i_{j}} x\right\|<\varepsilon$ for every $1 \leq j \leq r$. Hence $\#\left\{1 \leq i \leq m ;\left\|T^{i} x\right\|<\varepsilon\right\} \geq m(1-\varepsilon)$. In the same way, the set of operators $T$ such that $\#\left\{1 \leq i \leq n ;\left\|T^{i} x\right\|>1 / \varepsilon\right\} \geq n(1-\varepsilon)$ is SOT-open in $\mathfrak{B}_{M}(\mathcal{H})$. This proves Fact 2.38 .

The last step of the proof of Proposition 2.37 is the following fact.

Fact 2.39. - The set $\mathrm{DDCH}_{M}(\mathcal{H})$ is dense in $\left(\mathfrak{B}_{M}(\mathcal{H}), \mathrm{SOT}^{*}\right)$.

Proof. - By a result of [29], every ergodic operator on $\mathcal{H}$ is densely distributionally chaotic. Since G-MIX $(\mathcal{H})$ is dense in $\left(\mathfrak{B}_{M}(\mathcal{H})\right.$, $\left.\mathrm{SOT}^{*}\right)$ by Corollary 2.12, it immediately follows that $\operatorname{DDCH}_{M}(\mathcal{H})$ is dense in $\left(\mathfrak{B}_{M}(\mathcal{H})\right.$, $\left.\mathrm{SOT}^{*}\right)$.

By Facts 2.38 and 2.39, the proof of Proposition 2.37 is now complete.

Since $\operatorname{DDCH}(\mathcal{H}) \subseteq \operatorname{DCH}(\mathcal{H})$, and since any operator $T \in \operatorname{DDCH}(\mathcal{H}) \cap \mathrm{HC}(\mathcal{H})$ satisfies $c(T)=1$, Proposition 2.37 has the following immediate consequences:

Corollary 2.40. - For any $M>1$, the set $\mathrm{DCH}_{M}(\mathcal{H})$ of distributionally chaotic operators in $\mathfrak{B}_{M}(\mathcal{H})$ is comeager in $\left(\mathfrak{B}_{M}(\mathcal{H})\right.$, $\left.\mathrm{SOT}^{*}\right)$.

Corollary 2.41. - For any $M>1$, the set $\operatorname{cMAX}_{M}(\mathcal{H})$ of operators $T \in \mathrm{HC}_{M}(\mathcal{H})$ with $c(T)=1$ is comeager in $\left(\mathrm{HC}_{M}(\mathcal{H}), \mathrm{SOT}^{*}\right)$. 
There is an alternative approach for proving the comeagerness of $\operatorname{DDCH}_{M}(\mathcal{H})$, which relies on the next proposition, combined with the Kuratowski-Ulam Theorem.

Proposition 2.42. - Let $M>1$. For every vector $x \in \mathcal{H} \backslash\{0\}$, the set

$$
\mathfrak{G}^{x}=\left\{T \in \mathfrak{B}_{M}(\mathcal{H}) ; x \text { is a distributionally irregular vector for } T\right\}
$$

is comeager in $\left(\mathrm{HC}_{M}(\mathcal{H}), \mathrm{SOT}^{*}\right)$.

By the Kuratowski-Ulam Theorem, Proposition 2.42 implies that the set

$$
\mathfrak{G}:=\left\{(T, x) \in \mathfrak{B}_{M}(\mathcal{H}) \times \mathcal{H} ; x \text { is a distributionally irregular vector for } T\right\}
$$

is comeager in $\left(\mathfrak{B}_{M}(\mathcal{H}), \mathrm{SOT}^{*}\right) \times \mathcal{H}$; and this, in turn, implies that the set

$$
\left\{T \in \mathfrak{B}_{M}(\mathcal{H}) ; \forall^{*} x \in \mathcal{H} \text { is distributionally irregular for } T\right\}
$$

is comeager in $\left(\mathfrak{B}_{M}(\mathcal{H}), \mathrm{SOT}^{*}\right)$. Here, " $\forall * x \in \mathcal{H}$ " means "for quasi-all $x \in \mathcal{H}$ in the Baire category sense". This shows that $\operatorname{DDCH}_{M}(\mathcal{H})$ is comeager in $\mathfrak{B}_{M}(\mathcal{H})$.

For the proof of Proposition 2.42, we will need the following lemma.

Lemma 2.43. - Let $T$ be an ergodic operator on $\mathcal{H}$, and let $m$ be a $T$-invariant ergodic measure with full support for $T$. For every $\varepsilon, R>0$, one can find two other ergodic measures $\mu$ and $\nu$ for $T$, both with full support, such that $\mu(\bar{B}(0, R))>1-\varepsilon$ and $\nu(\bar{B}(0, R))<\varepsilon$ respectively.

Proof. - The proof relies on a dilation argument already used several times in [29. This argument has been essentially given at the end of the proof of Theorem 2.29, but we repeat it anyway. For any $\rho>0$, let $m_{\rho}$ be the measure on $\mathcal{H}$ defined by $m_{\rho}(C)=m(\rho C)$ for any Borel subset $C$ of $\mathcal{H}$. We have $m_{\rho}(\bar{B}(0, R))=m(\bar{B}(0, \rho R))$. All these measures $m_{\rho}$ are ergodic for $T$ and have full support. Moreover, $m(\bar{B}(0, \rho R)) \rightarrow m(\mathcal{H})=1$ as $\rho \rightarrow \infty$ and $m(\bar{B}(0, \rho R)) \rightarrow m(\{0\})=0$ as $\rho \rightarrow 0$ (that $m(\{0\})$ is necessarily equal to 0 follows from the ergodicity of $m$ with respect to $T$ and the fact that $\left.m \neq \delta_{0}\right)$. So there exist $\rho_{1}$, $\rho_{2}>0$ such that $\mu=m_{\rho_{1}}$ and $\nu=m_{\rho_{2}}$ satisfy $\mu(\bar{B}(0, R))>1-\varepsilon$ and $\nu(\bar{B}(0, R))<\varepsilon$.

Proof of Proposition 2.42. - Let us consider the following two subsets of $\mathfrak{B}_{M}(\mathcal{H})$ :

$$
\begin{gathered}
\mathfrak{G}_{\infty}^{x}:=\left\{T \in \mathfrak{B}_{M}(\mathcal{H}) ; \forall R>0: \underline{\text { dens }} \mathcal{N}_{T}(x, \bar{B}(0, R))=0\right\}, \quad \text { and } \\
\mathfrak{G}_{0}^{x}:=\left\{T \in \mathfrak{B}_{M}(\mathcal{H}) ; \forall r>0: \underline{\text { dens }} \mathcal{N}_{T}(x, \mathcal{H} \backslash \bar{B}(0, r))=0\right\} .
\end{gathered}
$$

It is not difficult to see that

$$
\mathfrak{G}_{\infty}^{x} \cap \mathfrak{G}_{0}^{x} \subseteq \mathfrak{G}^{x} .
$$

Indeed, if $T \in \mathfrak{G}_{\infty}^{x}$, then one can find a set $A \subseteq \mathbb{N}$ with $\overline{\operatorname{dens}}(A)=1$ such that $\left\|T^{i} x\right\| \rightarrow \infty$ as $i \rightarrow \infty$ along $A$; whereas if $T \in \mathfrak{G}_{0}^{x}$, one can find a set $B \subseteq \mathbb{N}$ with $\overline{\operatorname{dens}}(B)=1$ such that $\left\|T^{i} x\right\| \rightarrow 0$ as $i \rightarrow \infty$ along $B$. So it is enough to show that $\mathfrak{G}_{\infty}^{x}$ and $\mathfrak{G}_{0}^{x}$ are both comeager in $\left(\mathfrak{B}_{M}(\mathcal{H}), \mathrm{SOT}^{*}\right)$. We will actually concentrate on $\mathfrak{G}_{\infty}^{x}$ only, the proof for $\mathfrak{G}_{0}^{x}$ being completely similar.

For any $\varepsilon, R>0$, we introduce the set

$$
\mathfrak{H}_{\varepsilon, R}^{x}=\bigcap_{k \geq 1} \bigcup_{n \geq k}\left\{T \in \mathfrak{B}_{M}(\mathcal{H}) ; \#\left\{1 \leq i \leq n ;\left\|T^{i} x\right\|<R\right\}<n \varepsilon\right\} .
$$

Reasoning as in the proof of Fact 2.38, we observe that each set $\mathfrak{H}_{\varepsilon, R}^{x}$ is a $G_{\delta}$ subset of $\left(\mathfrak{B}_{M}(\mathcal{H})\right.$, SOT $)$. We also have:

Fact 2.44. - Each set $\mathfrak{H}_{\varepsilon, R}^{x}$ is dense in $\left(\mathfrak{B}_{M}(\mathcal{H}), \mathrm{SOT}^{*}\right)$. 
Proof of Fact 2.44 - We will use the density of $\mathrm{G}_{-} \mathrm{MIX}_{M}(\mathcal{H})$ in $\left(\mathfrak{B}_{M}(\mathcal{H})\right.$, $\left.\mathrm{SOT}^{*}\right)$, proved in Corollary 2.12 above.

Let $\mathfrak{U}$ be a non-empty open subset of $\left(\mathfrak{B}_{M}(\mathcal{H})\right.$, SOT$\left.^{*}\right)$. By Corollary 2.12, we know that $\operatorname{G-MIX}_{M^{\prime}}(\mathcal{H})$ is dense in $\left(\mathfrak{B}_{M^{\prime}}(\mathcal{H})\right.$, SOT* $)$ for every $1<M^{\prime}<M$. Since $\bigcup_{M^{\prime}<M} \mathfrak{B}_{M^{\prime}}(\mathcal{H})$ is obviously dense in $\mathfrak{B}_{M}(\mathcal{H})$, it follows that $\mathfrak{U}$ contains an operator $T$ which is mixing in the Gaussian sense and satisfies $\|T\|<M$. The operator $T$ is in particular ergodic. By Lemma 2.43, $T$ admits an ergodic measure with full support $\nu$ such that $\nu(\bar{B}(0,2 R))<\varepsilon$. Birkhoff's ergodic theorem then implies that the set

$$
\mathcal{E}:=\left\{y \in \mathcal{H} ; \limsup _{n \rightarrow \infty} \frac{1}{n} \#\left\{1 \leq i \leq n ;\left\|T^{i} y\right\| \leq 2 R\right\}<\varepsilon\right\}
$$

is dense in $\mathcal{H}$.

Let now $\delta>0$ be a small positive number, to be fixed later on in the proof. Since $x \neq 0$, the density of $\mathcal{E}$ in $\mathcal{H}$ implies the existence of an isomorphism $L$ of $\mathcal{H}$ with the following properties: $L x \in \mathcal{E},\|I-L\|<\delta$, and $\left\|I-L^{-1}\right\|<\delta$. Consider now the operator $S=L^{-1} T L$. Since $\|T\|<M$, we have $\|S\|<M$ if $\delta$ is sufficiently small. Also, $S$ belongs to $\mathfrak{U}$ as soon as $\delta$ is sufficiently small. We thus fix $\delta>0$ such that these two conditions are satisfied. It now remains to prove that $S$ belongs to $\mathfrak{H}_{\varepsilon, R}^{x}$, which will conclude our proof that $\mathfrak{H}_{\varepsilon, R}^{x}$ is dense in $\left(\mathfrak{B}_{M}(\mathcal{H})\right.$, SOT $\left.^{*}\right)$.

For every $n \geq 1$, we have $L S^{n} x=T^{n} L x$. Since $L x$ belongs to $\mathcal{E}$, it follows that

$$
\limsup _{n \rightarrow \infty} \frac{1}{n} \#\left\{1 \leq i \leq n ;\left\|L S^{i} x\right\| \leq 2 R\right\}<\varepsilon .
$$

Observe now that if $\left\|S^{i} x\right\| \leq R$, then $\left\|L S^{i} x\right\| \leq\|L\|\left\|S^{i} x\right\| \leq 2 R$ (as soon as $\delta \leq 2$, of course). It follows that

$$
\#\left\{1 \leq i \leq n ;\left\|S^{i} x\right\| \leq R\right\} \leq \#\left\{1 \leq i \leq n ;\left\|L S^{i} x\right\| \leq 2 R\right\}<n \varepsilon
$$

for all sufficiently large $n$. Hence $S$ belongs to $\mathfrak{H}_{\varepsilon, R}^{x}$, which concludes the proof of Fact 2.44,

The two facts above imply that all the sets $\mathfrak{H}_{\varepsilon, R}^{x}$ are comeager (in fact, dense $G_{\delta}$ ) in $\left(\mathfrak{B}_{M}(\mathcal{H})\right.$, SOT $\left.^{*}\right)$. Since

$$
\mathfrak{G}_{\infty}^{x}=\bigcap_{\varepsilon \in \mathbb{Q}_{+}^{*}} \bigcap_{R \in \mathbb{Q}_{+}^{*}} \mathfrak{H}_{\varepsilon, R}^{x},
$$

it follows that $\mathfrak{G}_{\infty}^{x}$ is comeager as well. The case of $\mathfrak{G}_{0}^{x}$ being exactly similar, the proof of Proposition 2.42 is now complete.

2.8. Summary. - Let us summarize the results obtained so far: for any $M>1$, an SOT*-typical hypercyclic operator $T$

- is topologically weakly mixing but not topologically mixing;

- has empty point spectrum, and hence is not chaotic;

- has no non-trivial invariant measure, hence is not $\mathcal{U}$-frequently hypercyclic, and $a$ fortiori not ergodic;

- but is densely distributionally chaotic and hence satisfies $c(T)=1$.

We shall see in Section 4 below that the picture changes drastically when we consider SOT-typical elements of some natural classes of upper-triangular operators with respect to a given orthonormal basis of $\mathcal{H}$. 


\section{Descriptive set-theoretic issues}

In what follows, we fix $M>1$. We have seen in the previous section that $\mathrm{CH}_{M}(\mathcal{H})$, $\operatorname{TMIX}_{M}(\mathcal{H})$ and $\operatorname{UFHC}_{M}(\mathcal{H})$ are meager in $\left(\mathfrak{B}_{M}(\mathcal{H})\right.$, SOT* $)$. In this section, we are going to show that these classes of operators are also Borel in $\mathfrak{B}_{M}(\mathcal{H})$ with respect to SOT and SOT*, and we will discuss their exact descriptive complexity in some details. Moreover, we will show that some natural classes of operators defined by dynamical properties are non-Borel in $\mathfrak{B}_{M}(\mathcal{H})$.

Recall the standard notations for Borel classes: $\boldsymbol{\Sigma}_{1}^{0}=$ open, $\boldsymbol{\Pi}_{1}^{0}=$ closed, $\boldsymbol{\Sigma}_{2}^{0}=F_{\sigma}$, $\boldsymbol{\Pi}_{2}^{0}=G_{\delta}$ and so on. We refer the reader to [37] for more information on the Borel hierarchy.

3.1. Complexity of the families $\operatorname{TMIX}_{M}(\mathcal{H}), \mathbf{C H}_{M}(\mathcal{H}), \operatorname{UFHC}_{M}(\mathcal{H}) \cap \mathbf{C H}_{M}(\mathcal{H})$ and $\operatorname{UFHC}_{M}(\mathcal{H})$. - The following fact will allow us to concentrate mainly on the topology SOT*.

Lemma 3.1. - The identity map id $:\left(\mathfrak{B}_{M}(\mathcal{H}), \mathrm{SOT}\right) \rightarrow\left(\mathfrak{B}_{M}(\mathcal{H}), \mathrm{SOT}^{*}\right)$ is Baire 1 ; in other words, any SOT*-open subset of $\mathfrak{B}_{M}(\mathcal{H})$ is SOT- $\Sigma_{2}^{0}$. Therefore, for every countable ordinal $\xi$, any $\mathrm{SOT}^{*}-\boldsymbol{\Sigma}_{\xi}^{0}$ subset of $\mathfrak{B}_{M}(\mathcal{H})$ is $\mathrm{SOT}-\Sigma_{\xi+1}^{0}$ and any $\mathrm{SOT}^{*}-\Pi_{\xi}^{0}$ set is $\mathrm{SOT}-\Pi_{\xi+1}^{0}$.

Proof. - Since $\left(\mathfrak{B}_{M}(\mathcal{H}), \mathrm{SOT}^{*}\right)$ is second-countable, it is enough to show that any basic SOT*-open set is SOT- $\boldsymbol{\Sigma}_{2}^{0}$. Therefore, we just have to check that if $x, a \in \mathcal{H}$ and $\varepsilon>0$, then the set $\mathfrak{U}:=\left\{T \in \mathfrak{B}_{M}(\mathcal{H}) ;\left\|T^{*} x-a\right\|<\varepsilon\right\}$ is SOT- $F_{\sigma}$. But this is clear since

$$
T \in \mathfrak{U} \Longleftrightarrow \exists k \in \mathbb{N}\left(\forall h \in \mathcal{H},\|h\| \leq 1:|\langle x, T h\rangle-\langle a, h\rangle| \leq \varepsilon-\frac{1}{k}\right)
$$

and the condition under brackets is SOT-closed.

3.1.1. Complexity of $\operatorname{TMIX}_{M}(\mathcal{H})$. - The complexity of $\operatorname{TMIX}_{M}(\mathcal{H})$ is given by the following proposition:

Proposition 3.2. - The set $\operatorname{TMIX}_{M}(\mathcal{H})$ is a $\mathbf{\Pi}_{4}^{0}$ subset of $\left(\mathfrak{B}_{M}(\mathcal{H}), \mathrm{SOT}\right)$, and a "true" $\boldsymbol{\Pi}_{3}^{0}$ subset of $\left(\mathfrak{B}_{M}(\mathcal{H})\right.$, SOT $\left.^{*}\right)$, i.e. a $\boldsymbol{\Pi}_{3}^{0}$ set which is not $\boldsymbol{\Sigma}_{3}^{0}$.

Proof. - Let us first show that $\operatorname{TMIX}_{M}(\mathcal{H})$ is $\Pi_{3}^{0}$ with respect to $\mathrm{SOT}^{*}$, and hence (by Lemma 3.1) $\Pi_{4}^{0}$ with respect to SOT. Let $\left(B_{p}\right)_{p \geq 1}$ be a countable family of closed balls of $\mathcal{H}$ whose interiors form a basis of open sets for $\mathcal{H}$. Then an operator $T \in \mathfrak{B}_{M}(\mathcal{H})$ is topologically mixing if and only if

$$
\forall p, q \geq 1 \exists N \in \mathbb{N} \forall n \geq N: T^{n}\left(B_{p}\right) \cap B_{q} \neq \emptyset .
$$

For each fixed data $(p, q, n)$, the condition " $T^{n}\left(B_{p}\right) \cap B_{q} \neq \emptyset$ " is SOT*-closed, by weak compactness of $B_{p}$, weak closedness of $B_{q}$ and continuity of the map $(T, x) \mapsto T^{n} x$ from $\left(\mathfrak{B}_{M}(\mathcal{H}), \operatorname{SOT}^{*}\right) \times\left(B_{p}, w\right)$ into $(\mathcal{H}, w)$. This shows that $\operatorname{TMIX}_{M}(\mathcal{H})$ is $\Pi_{3}^{0}$ in $\left(\mathfrak{B}_{M}(\mathcal{H})\right.$, SOT $\left.^{*}\right)$.

In order to show that $\operatorname{TMIX}_{M}(\mathcal{H})$ is a true $\Pi_{3}^{0}$ subset of $\left(\mathfrak{B}_{M}(\mathcal{H})\right.$, SOT* $)$, we assume that $\mathcal{H}=\ell^{2}(\mathbb{N})$ and we use weighted backward shifts on $\mathcal{H}$. It is well-known that a weighted backward shift $B_{\boldsymbol{\omega}}$ on $\mathcal{H}$ is topologically mixing if and only if the weight sequence $\boldsymbol{\omega}=\left(\omega_{k}\right)_{k \geq 1}$ satisfies

$$
\lim _{n \rightarrow \infty}\left|\omega_{1} \cdots \omega_{n}\right|=\infty
$$


Let us denote by $\mathcal{S}$ the set of all sequences of positive integers $s=\left(s_{k}\right)_{k \geq 1}$ such that $s_{k+1} \leq M s_{k}$ for all $k \geq 1$. This is a closed subset of the Polish space $\mathbb{N}^{\mathbb{N}}$, and hence a Polish space as well. We need the following fact.

Fact 3.3. - The set $\mathcal{S}_{\infty}:=\left\{s \in \mathcal{S} ; s_{k} \rightarrow \infty\right.$ as $\left.k \rightarrow \infty\right\}$ is a true $\Pi_{3}^{0}$ set in $\mathcal{S}$.

Proof of Fact 3.3, - It is known (see e.g. [37, Section 23.A]) that $\mathcal{N}_{\infty}:=\left\{\alpha \in \mathbb{N}^{\mathbb{N}} ; \alpha_{k} \rightarrow\right.$ $\infty\}$ is a true $\Pi_{3}^{0}$ set in $\mathbb{N}^{\mathbb{N}}$. So we just need to find a continuous map $\Phi: \mathbb{N}^{\mathbb{N}} \rightarrow \mathcal{S}$ such that $\Phi^{-1}\left(\mathcal{S}_{\infty}\right)=\mathcal{N}_{\infty}$. In other words, our goal is to associate to each $\alpha \in \mathbb{N}^{\mathbb{N}}$ another sequence $s \in \mathbb{N}^{\mathbb{N}}$ in such a way that $s_{k} \rightarrow \infty$ exactly when $\alpha_{k} \rightarrow \infty$ and, additionally, $s_{k+1} \leq M s_{k}$ for all $k \geq 1$; and this needs to be done in a continuous fashion. For any $\alpha \in \mathbb{N}^{\mathbb{N}}$, we define $s=s(\alpha)$ as follows: $s_{1}=\alpha_{1}$ and, for every $k \geq 1$,

$$
s_{k+1}= \begin{cases}\alpha_{k+1} & \text { if } \alpha_{k+1} \leq M s_{k} \\ M s_{k} & \text { if } \alpha_{k+1}>M s_{k}\end{cases}
$$

It is obvious that $s_{k+1} \leq M s_{k}$ for all $k \geq 1$ and that the map $\alpha \mapsto s(\alpha)$ is continuous. Moreover, since $M>1$, it is straightforward to check that $\alpha_{k} \rightarrow \infty$ if and only if $s_{k} \rightarrow$ $\infty$.

Going back to the proof of Proposition 3.2, we associate to each $s \in \mathcal{S}$ a weight sequence $\boldsymbol{\omega}(s)$ defined as follows:

$$
\omega_{1}(s)=1 \quad \text { and } \quad \omega_{k+1}(s)=\frac{s_{k+1}}{s_{k}} \quad \text { for every } k \geq 1 .
$$

Since $s \in \mathcal{S}$, we have $0<\omega_{k}(s) \leq M$ for all $k \geq 1$, so that the weighted shift $B_{\boldsymbol{\omega}(s)}$ on $\mathcal{H}$ satisfies $\left\|B_{\boldsymbol{\omega}(s)}\right\| \leq M$. Moreover, the map $s \mapsto \boldsymbol{\omega}(s)$ is clearly continuous from $\mathcal{S}$ into $\mathbb{R}^{\mathbb{N}}$, and hence the map $s \mapsto B_{\boldsymbol{\omega}(s)}$ is continuous from $\mathcal{S}$ into $\left(\mathfrak{B}_{M}(\mathcal{H})\right.$, SOT $\left.^{*}\right)$. Finally, since $\omega_{1}(s) \cdots \omega_{k}(s)=s_{k} / s_{1}$ for all $k \geq 1$, the shift $B_{\boldsymbol{\omega}(s)}$ is topologically mixing if and only if $s_{k} \rightarrow \infty$. We have thus constructed a continuous map $\Phi: \mathcal{S} \rightarrow \mathfrak{B}_{M}(\mathcal{H})$ such that $\Phi^{-1}\left(\operatorname{TMIX}_{M}(\mathcal{H})\right)=\mathcal{S}_{\infty}$, which proves that $\operatorname{TMIX}_{M}(\mathcal{H})$ is a true $\Pi_{3}^{0}$ subset of $\left(\mathfrak{B}_{M}(\mathcal{H}), \mathrm{SOT}^{*}\right)$ by Fact 3.3 .

3.1.2. Complexity of $\mathrm{CH}_{M}(\mathcal{H})$. - We now consider the class of chaotic operators on $\mathcal{H}$.

Proposition 3.4. - The set $\mathrm{CH}_{M}(\mathcal{H})$ is a $\Pi_{4}^{0}$ subset of $\left(\mathfrak{B}_{M}(\mathcal{H})\right.$, SOT), and a true $\Pi_{3}^{0}$ subset of $\left(\mathfrak{B}_{M}(\mathcal{H})\right.$, SOT $\left.^{*}\right)$.

Proof. - Let us first show that $\mathrm{CH}_{M}(\mathcal{H})$ is $\Pi_{3}^{0}$ with respect to SOT* and hence $\boldsymbol{\Pi}_{4}^{0}$ with respect to SOT. Let $\left(B_{p}\right)_{p \geq 1}$ be a countable family of closed balls whose interiors form a basis of open sets for $\mathcal{H}$. By definition, an operator $T \in \mathfrak{B}_{M}(\mathcal{H})$ is chaotic if and only if it is hypercyclic and each ball $B_{p}$ contains a periodic point for $T$. In other words:

$$
T \in \mathrm{CH}_{M}(\mathcal{H}) \Longleftrightarrow T \in \mathrm{HC}_{M}(\mathcal{H}) \text { and } \forall p \in \mathbb{N} \exists N \in \mathbb{N}:\left(\exists x \in B_{p}: T^{N} x=x\right) \text {. }
$$

For each fixed pair $(p, N)$, the condition under brackets is SOT*-closed by continuity of the map $(T, x) \mapsto T^{N} x$ and weak compactness of the ball $B_{p}$. Therefore, the second half of the condition on the right hand side of the above display defines a $\boldsymbol{\Pi}_{3}^{0}$ set; and since $\mathrm{HC}_{M}(\mathcal{H})$ is $G_{\delta}$, it follows that $\mathrm{CH}_{M}(\mathcal{H})$ is $\Pi_{3}^{0}$ in $\left(\mathfrak{B}_{M}(\mathcal{H})\right.$, SOT ${ }^{*}$.

The proof that $\mathrm{CH}_{M}(\mathcal{H})$ is a true $\Pi_{3}^{0}$ set is a little bit more involved. We will use the so-called Kalisch operators, introduced by Kalisch in [36], which display interesting 
dynamical properties (see for instance [7, Section 5.5.3]). They are defined as follows. Let $T: L^{2}(0,2 \pi) \rightarrow L^{2}(0,2 \pi)$ be the operator defined for every $f \in L^{2}(0,2 \pi)$ by

$$
T f(\theta)=e^{i \theta} f(\theta)-\int_{0}^{\theta} i e^{i t} f(t) d t, \quad \theta \in(0,2 \pi) .
$$

A simple computation shows that for any $\lambda=e^{i \alpha} \in \mathbb{T} \backslash\{1\}$, the function $f_{\lambda}:=\mathbf{1}_{(\alpha, 2 \pi)}$ is an eigenvector of $T$ associated to the eigenvalue $\lambda$, and that $\operatorname{ker}(T-\lambda)=\operatorname{span}\left[f_{\lambda}\right]$. Note also that the map $\lambda \mapsto f_{\lambda}$ is continuous from $\mathbb{T} \backslash\{1\}$ into $L^{2}(0,2 \pi)$. In particular, if $\Lambda \neq \emptyset$ is any compact subset of $\mathbb{T} \backslash\{1\}$, the closed subspace $\mathcal{H}_{\Lambda}$ of $L^{2}(0,2 \pi)$ spanned by the functions $f_{\lambda}, \lambda \in \Lambda$, is $T$-invariant. The Kalisch operator associated to $\Lambda$ is the operator $T_{\Lambda}: \mathcal{H}_{\Lambda} \rightarrow \mathcal{H}_{\Lambda}$ induced by $T$ on $\mathcal{H}_{\Lambda}$. These operators $T_{\Lambda}$ have the following properties.

- The spectrum of $T_{\Lambda}$ is exactly equal to $\Lambda$.

- The operator $T_{\Lambda}$ is hypercyclic if and only if $\Lambda$ is a perfect set, in which case $T_{\Lambda}$ is actually ergodic in the Gaussian sense.

Recall that a perfect set is a non-empty compact set without isolated points.

It is easy to deduce from these properties the following characterization of compact subsets $\Lambda$ of $\mathbb{T} \backslash\{1\}$ such that $T_{\Lambda}$ is chaotic. We denote by $\Omega$ the subset of $\mathbb{T}$ consisting of all roots of unity.

Fact 3.5. - Let $\Lambda$ be a perfect subset of $\mathbb{T} \backslash\{1\}$. Then the operator $T_{\Lambda}$ is chaotic if and only if $\Omega \cap \Lambda$ is dense in $\Lambda$.

Proof of Fact 3.5. - Since the map $\lambda \mapsto f_{\lambda}$ is continuous and $f_{\lambda}$ is a periodic point of $T_{\Lambda}$ if $\lambda \in \Omega \cap \Lambda$, it is clear that $\operatorname{Per}\left(T_{\Lambda}\right)$ is dense in $\mathcal{H}_{\Lambda}$ if $\Omega \cap \Lambda$ is dense in $\Lambda$. Since $\Lambda$ is assumed to be perfect, $T_{\Lambda}$ is hypercyclic, and hence chaotic. Conversely, assume that $\Omega \cap \Lambda$ is not dense in $\Lambda$ and choose $\lambda \in \Lambda \backslash \Lambda_{0}$, where $\Lambda_{0}=\overline{\Omega \cap \Lambda}$. Then $f_{\lambda}$ is not an eigenvector of $T_{\Lambda_{0}}$ since $\sigma\left(T_{\Lambda_{0}}\right)=\Lambda_{0}$. Since however $f_{\lambda}$ is an eigenvector of $T$, this means that $f_{\lambda}$ does not belong to $\mathcal{H}_{\Lambda_{0}}=\overline{\operatorname{span}}\left[f_{\xi} ; \xi \in \Lambda_{0}\right]$, i.e. that $f_{\lambda}$ does not belong to $\overline{\operatorname{span}}\left[f_{\xi} ; \xi \in \Omega \cap \Lambda\right]$. But since $\sigma\left(T_{\Lambda}\right)=\Lambda$ and $\operatorname{ker}(T-\xi)=\operatorname{span}\left[f_{\xi}\right]$ for every $\xi \in \mathbb{T} \backslash\{1\}$, we have

$$
\operatorname{span}\left[f_{\xi} ; \xi \in \Omega \cap \Lambda\right]=\operatorname{span}\left[\bigcup_{\xi \in \Omega \cap \Lambda} \operatorname{ker}\left(T_{\Lambda}-\xi\right)\right]=\operatorname{Per}\left(T_{\Lambda}\right) .
$$

So $\operatorname{Per}\left(T_{\Lambda}\right)$ is not dense in $\mathcal{H}_{\Lambda}$ and $T_{\Lambda}$ is not chaotic.

Here is now a purely descriptive set-theoretic fact, which is certainly well-known but for which we were unable to locate a reference. Here and afterwards, given a compact metric space $E$, we denote by $\mathcal{K}(E)$ the space of all non-empty compact subsets of $E$ endowed with its usual topology, and by $\mathcal{K}_{\text {perf }}(E)$ the set of all perfect subsets of $E$. Recall that $\mathcal{K}(E)$ is compact metrizable, and that $\mathcal{K}_{\text {perf }}(E)$ is a $G_{\delta}$ subset of $\mathcal{K}(E)$. So $\mathcal{K}_{\text {perf }}(E)$ is a Polish space. See [37] or [51 for more details.

Fact 3.6. - Let $E \subseteq \mathbb{T}$ be a perfect set such that $\Omega \cap E$ is dense in $E$. Then the set $\mathcal{W}:=\left\{\Lambda \in \mathcal{K}_{\text {perf }}(E) ; \Omega \cap \Lambda\right.$ is dense in $\left.\Lambda\right\}$ is a true $\Pi_{3}^{0}$ set in $\mathcal{K}_{\text {perf }}(E)$.

Proof of Fact [3.6. - Since $\mathcal{K}_{\text {perf }}(E)$ is $G_{\delta}$ in $\mathcal{K}(E)$, it is in fact enough to show that $\mathcal{W}$ is a true $\Pi_{3}^{0}$ set in $\mathcal{K}(E)$. That $\mathcal{W}$ is $\Pi_{3}^{0}$ is easy to check. In order to show that it is a true $\boldsymbol{\Pi}_{3}^{0}$ set, we proceed as follows. 
Let us denote by $\mathbf{C}$ the Cantor space $\{0,1\}^{\mathbb{N}}$, and by $\mathbf{Q}$ the set of all "rationals" of $\mathbf{C}$, i.e. $\mathbf{Q}=\left\{\alpha=(\alpha(i))_{i \geq 1} \in \mathbf{C} ; \alpha(i)\right.$ is eventually 0\}. It is known (see [37, Section 23.A]) that the set

$$
W:=\left\{\bar{\alpha}=\left(\alpha_{n}\right)_{n \geq 1} \in \mathbf{C}^{\mathbb{N}} ; \forall n \geq 1: \alpha_{n} \in \mathbf{Q}\right\}
$$

is a true $\Pi_{3}^{0}$ set in $\mathbf{C}^{\mathbb{N}}$. So it is enough to find a continuous map $\Phi: \mathbf{C}^{\mathbb{N}} \rightarrow \mathcal{K}(E)$ such that $\Phi^{-1}(\mathcal{W})=W$.

We first note that if $M \subseteq E$ is a perfect set such that $\Omega \cap M$ is dense in $M$, then $\mathcal{W} \cap \mathcal{K}(M)$ is not $G_{\delta}$ in $\mathcal{K}(M)$. Indeed, on the one hand $\mathcal{W} \cap \mathcal{K}(M)$ is easily seen to be dense in $\mathcal{K}(M)$ because $M$ is perfect and $\Omega \cap M$ is dense in $M$; and on the other hand $\mathcal{W} \cap \mathcal{K}(M)$ is meager in $\mathcal{K}(M)$ because it is disjoint from the $G_{\delta}$ set $\mathcal{K}(M \backslash \Omega)$, which is dense in $\mathcal{K}(M)$ because $M$ is perfect and $\Omega$ is countable. Hence $\mathcal{W} \cap \mathcal{K}(M)$ cannot be $G_{\delta}$ in $\mathcal{K}(M)$ by the Baire Category Theorem. By Wadge's Lemma (see [37, Th. 21.14]) applied to the two Borel sets $\mathcal{W} \cap \mathcal{K}(M)$ and $\mathbf{C} \backslash \mathbf{Q}$, it follows that for any perfect set $M$ as above, $\mathcal{W} \cap \mathcal{K}(M)$ is " $\Sigma_{2}^{0}$-hard", i.e. one can find a continuous map $\varphi: \mathbf{C} \rightarrow \mathcal{K}(M)$ such that $\varphi^{-1}(\mathcal{W} \cap \mathcal{K}(M))=\mathbf{Q}$.

Now let us choose a sequence $\left(M_{n}\right)_{n \geq 1}$ of pairwise disjoint perfect subsets of $E$ such that $\Omega \cap M_{n}$ is dense in $M_{n}$ for every $n \geq 1$ and the sets $M_{n}$ accumulate to some point $a \in E$, which means that every neighborhood of $a$ contains all but finitely many of the sets $M_{n}$. This is possible because $E$ is perfect and $\Omega \cap E$ is dense in $E$. For each $n \geq 1$, let $\varphi_{n}: \mathbf{C} \rightarrow \mathcal{K}\left(M_{n}\right)$ be a continuous map such that $\varphi_{n}^{-1}\left(\mathcal{W} \cap \mathcal{K}\left(M_{n}\right)\right)=\mathbf{Q}$. One can then define a map $\Phi: \mathbf{C}^{\mathbb{N}} \rightarrow \mathcal{K}(E)$ by setting

$$
\Phi(\bar{\alpha}):=\{a\} \cup \bigcup_{n=1}^{\infty} \varphi_{n}\left(\alpha_{n}\right) \quad \text { for every } \bar{\alpha}=\left(\alpha_{n}\right) \in \mathbf{C}^{\mathbb{N}} .
$$

Since the sets $M_{n}$ accumulate to $a$, it is clear that each $\Phi(\bar{\alpha})$ is indeed a compact subset of $\mathbb{T}$ and that the map $\Phi$ is continuous. Moreover, it is equally clear that $\Phi(\bar{\alpha})$ is perfect if and only if all the sets $\varphi_{n}\left(\alpha_{n}\right)$ are perfect, and that $\Omega \cap \Phi(\bar{\alpha})$ is dense in $\Phi(\bar{\alpha})$ if and only if $\Omega \cap \varphi_{n}\left(\alpha_{n}\right)$ is dense in $\varphi_{n}\left(\alpha_{n}\right)$ for every $n \geq 1$. Hence $\Phi(\bar{\alpha})$ belongs to $\mathcal{W}$ if and only if $\alpha_{n}$ belongs to $\varphi_{n}^{-1}\left(\mathcal{W} \cap \mathcal{K}\left(M_{n}\right)\right)=\mathbf{Q}$ for all $n \geq 1$. We have thus proved that

$$
\Phi^{-1}(\mathcal{W})=\left\{\bar{\alpha} ; \forall n \geq 1: \alpha_{n} \in \mathbf{Q}\right\}=W,
$$

which concludes the proof of Fact 3.6.

We need yet one more fact, which is a simple and certainly well-known consequence of the Michael Selection Theorem. The version of this theorem which we use here runs as follows (see [51, Section III.19]).

Let $X$ be a zero-dimensional compact space, and let $Y$ be a complete metric space. Let $\Phi: X \rightarrow \mathcal{F}(Y)$ be a lower semi-continuous map, where $\mathcal{F}(Y)$ denotes the set of all non-empty closed subsets of $Y$. Then $\Phi$ admits a continuous selection, i.e. there exists a continuous map $f: X \rightarrow Y$ such that $f(x)$ belongs to $\Phi(x)$ for every $x \in X$.

Fact 3.7. - Let $E$ be a zero-dimensional compact metric space. There exists a sequence $\left(\xi_{n}\right)_{n \geq 1}$ of continuous maps from $\mathcal{K}_{\text {perf }}(E)$ into $E$ such that for each $\Lambda \in \mathcal{K}_{\text {perf }}(E)$, the points $\xi_{n}(\Lambda), n \geq 1$, belong to $\Lambda$, are pairwise distinct, and the countable set $\left\{\xi_{n}(\Lambda) ; n \geq\right.$ $1\}$ is dense in $\Lambda$.

Proof of Fact 3.7. - Since $E$ is zero-dimensional, one can choose a countable basis $\left(V_{i}\right)_{i \geq 1}$ for the topology of $E$ consisting of (non-empty) clopen sets. For each $\Lambda \in \mathcal{K}_{\text {perf }}(E)$, let us 
denote by $i_{1}(\Lambda)<i_{2}(\Lambda)<\ldots$ the integers $i$ such that $\Lambda \cap V_{i} \neq \emptyset$ (since $\Lambda$ is perfect, there are infinitely many such integers $i$ ). As the $V_{i}$ are clopen sets, it is not hard to see that the functions $i_{n}, n \geq 1$, are locally constant. In particular, the map $\Phi: \Lambda \mapsto \Lambda \cap V_{i_{1}(\Lambda)}$ from $\mathcal{K}_{\text {perf }}(E)$ into $\mathcal{K}(E)$ is continuous. Since $E$ is zero-dimensional, $\mathcal{K}_{\text {perf }}(E)$ is zerodimensional as well, and one can apply the version of Michael's Selection Theorem quoted above to the map $\Phi$. This yields a continuous map $\Lambda \mapsto \xi_{1}(\Lambda)$ from $\mathcal{K}_{\text {perf }}(E)$ into $E$ such that $\xi_{1}(\Lambda)$ belongs to $\Lambda \cap V_{i_{1}(\Lambda)}$ for every $\Lambda \in \mathcal{K}_{\text {perf }}(E)$. Now, any perfect set $\Lambda \in \mathcal{K}_{\text {perf }}(E)$ satisfies $\Lambda \cap\left(V_{i_{2}(\Lambda)} \backslash\left\{\xi_{1}(\Lambda)\right\}\right) \neq \emptyset$; so there exists an integer $j$ such that $\xi_{1}(\Lambda) \in V_{j}$ and $V_{i_{2}(\Lambda)} \backslash V_{j} \neq \emptyset$. Moreover, if we denote by $j_{2}(\Lambda)$ the smallest such integer $j$ with these two properties, the map $\Lambda \mapsto j_{2}(\Lambda)$ is locally constant, and hence the map $\Lambda \mapsto \Lambda \cap\left(V_{i_{2}(\Lambda)} \backslash V_{j_{2}(\Lambda)}\right)$ is continuous from $\mathcal{K}_{\text {perf }}(E)$ into $\mathcal{K}(E)$. Applying Michael's Selection Theorem a second time, we obtain a continuous map $\xi_{2}: \mathcal{K}_{\text {perf }}(E) \rightarrow E$ such that $\xi_{2}(\Lambda) \in \Lambda \cap\left(V_{i_{2}(\Lambda)} \backslash V_{j_{2}(\Lambda)}\right)$ for every $\Lambda \in \mathcal{K}_{\text {perf }}(E)$; in particular, $\xi_{2}(\Lambda) \in \Lambda \cap V_{i_{2}(\Lambda)}$ and $\xi_{2}(\Lambda) \neq \xi_{1}(\Lambda)$. Continuing in this fashion, we obtain the required sequence $\left(\xi_{n}\right)_{n \geq 1}$.

We are now in position to prove that $\mathrm{CH}_{M}(\mathcal{H})$ is a true $\Pi_{3}^{0}$ set in $\left(\mathfrak{B}_{M}(\mathcal{H})\right.$, SOT* $)$. Let us first choose a compact set $E \subseteq \mathbb{T} \backslash\{1\}$ with the following properties: $E$ is perfect and zero-dimensional, $\Omega \cap E$ is dense in $E$ and, moreover, $\left\|T_{E}\right\| \leq M$. Such a compact set does exist. Indeed, the definition of the operator $T$ shows that the norm of the restriction of $T$ to the subspace $L^{2}(u, 2 \pi) \subseteq L^{2}(0,2 \pi)$ tends to 1 as $u \rightarrow 2 \pi$. Therefore, if $\Lambda_{0} \subseteq \mathbb{T} \backslash\{1\}$ is a non-trivial closed arc sufficiently close to 1 then $\left\|T_{\Lambda_{0}}\right\| \leq M$; so it is enough to take as $E$ any perfect set with empty interior contained in $\Lambda_{0}$ and such that $\Omega \cap E$ is dense in $E$.

Having fixed $E$ in this way, let $\left(\xi_{n}\right)_{n>1}$ be a sequence of continuous maps given by Fact 3.7. selecting a dense sequence of pairwise distinct points in each perfect set $\Lambda \subseteq E$. Note that if $\Lambda \in \mathcal{K}_{\text {perf }}(E)$, the points $\xi_{n}(\Lambda)$ are pairwise distinct and form a dense subset of $\Lambda$, so that the functions $f_{\xi_{n}(\Lambda)}$ are linearly independent and span a dense subspace of $\mathcal{H}_{\Lambda}$. Applying the Gram-Schmidt orthonormalization process to the sequence $\left(f_{\xi_{n}(\Lambda)}\right)_{n \geq 1}$, we obtain an orthonormal basis $\left(e_{n}(\Lambda)\right)_{n \geq 1}$ of $\mathcal{H}_{\Lambda}$ which depends continuously on $\Lambda$, that is, each map $\Lambda \mapsto e_{n}(\Lambda)$ is continuous from $\mathcal{K}_{\text {perf }}(E)$ into $L^{2}(0,2 \pi)$.

Let us now fix an orthonormal basis $\left(e_{n}\right)_{n \geq 1}$ of $\mathcal{H}$. For each $\Lambda \in \mathcal{K}_{\text {perf }}(E)$, denote by $U_{\Lambda}: \mathcal{H}_{\Lambda} \rightarrow \mathcal{H}$ the unitary operator defined by setting $U_{\Lambda} e_{n}(\Lambda)=e_{n}$ for every $n \geq 1$. Since $\left\|T_{E}\right\| \leq M$, one can define a map $\Phi: \mathcal{K}_{\text {perf }}(E) \rightarrow \mathfrak{B}_{M}(\mathcal{H})$ by setting

$$
\Phi(\Lambda):=U_{\Lambda} T_{\Lambda} U_{\lambda}^{-1} \quad \text { for every } \Lambda \in \mathcal{K}_{\text {perf }}(E) .
$$

Since $\Phi(\Lambda)$ and $T_{\Lambda}$ are unitarily isomorphic, $\Phi(\Lambda)$ is chaotic if and only if $T_{\Lambda}$ is, which holds true exactly when $\Omega \cap \Lambda$ is dense in $\Lambda$. Thus, we have

$$
\Phi^{-1}\left(\mathrm{CH}_{M}(\mathcal{H})\right)=\mathcal{W} .
$$

Fact 3.6 will allow us to conclude the proof, provided that we are able to show that the map $\Phi$ is continuous from $\mathcal{K}_{\text {perf }}(E)$ into $\left(\mathfrak{B}_{M}(\mathcal{H}), \mathrm{SOT}^{*}\right)$. This relies on the following observation. For every $\Lambda \in \mathcal{K}_{\text {perf }}(E)$, let us denote by $P_{\Lambda}: L^{2}(0,2 \pi) \rightarrow \mathcal{H}_{\Lambda}$ the orthogonal projection of $L^{2}(0,2 \pi)$ onto $\mathcal{H}_{\Lambda}$, and by $J_{\Lambda}: \mathcal{H}_{\Lambda} \rightarrow L^{2}(0,2 \pi)$ the canonical embedding of $\mathcal{H}_{\Lambda}$ into $L^{2}(0,2 \pi)$. In other words, $J_{\Lambda}=P_{\Lambda}^{*}$.

Claim 3.8. - For any $f \in L^{2}(0,2 \pi)$, the map $\Lambda \mapsto U_{\Lambda} P_{\Lambda} f$ is continuous from $\mathcal{K}_{\text {perf }}(E)$ into $\mathcal{H}$; and for every $x \in \mathcal{H}$, the map $\Lambda \mapsto J_{\Lambda} U_{\Lambda}^{-1} x$ is continuous from $\mathcal{K}_{\text {perf }}(E)$ into $L^{2}(0,2 \pi)$. 
Proof of Claim 3.8. Fix $f \in L^{2}(0,2 \pi)$. Since $P_{\Lambda} f=\sum_{n=1}^{\infty}\left\langle f, e_{n}(\Lambda)\right\rangle e_{n}(\Lambda)$, we have

$$
U_{\Lambda} P_{\Lambda} f=\sum_{n=1}^{\infty}\left\langle f, e_{n}(\Lambda)\right\rangle e_{n} .
$$

The maps $\Lambda \mapsto e_{n}(\Lambda), n \geq 1$, being continuous, it suffices to show that the convergence of the above series is uniform (with respect to $\Lambda$ ) on compact subsets of $\mathcal{K}_{\text {perf }}(E)$ in order to derive the continuity of the map $\Lambda \mapsto U_{\Lambda} P_{\Lambda} f$. Now, we have for any $N \geq 1$

$$
R_{N}(\Lambda):=\left\|\sum_{n>N}\left\langle f, e_{n}(\Lambda)\right\rangle e_{n}\right\|^{2}=\sum_{n>N}\left|\left\langle f, e_{n}(\Lambda)\right\rangle\right|^{2}=\left\|f-\sum_{n=1}^{N}\left\langle f, e_{n}(\Lambda)\right\rangle e_{n}(\Lambda)\right\|^{2} .
$$

In particular, the maps $\Lambda \mapsto R_{N}(\Lambda)$ are continuous. Since the sequence $\left(R_{N}\right)_{N \geq 1}$ is nonincreasing, it converges uniformly to 0 on compact subsets of $\mathcal{K}_{\text {perf }}(E)$ by Dini's Theorem. This proves the first part of Claim 3.8, The proof of the second part is exactly similar.

Let us now prove that $\Phi$ is continuous from $\mathcal{K}_{\text {perf }}(E)$ into $\left(\mathfrak{B}_{M}(\mathcal{H}), \mathrm{SOT}^{*}\right)$, which amounts to showing that for any $x \in \mathcal{H}$, the maps $\Lambda \mapsto \Phi(\Lambda) x$ and $\Lambda \mapsto \Phi(\Lambda)^{*} x$ are continuous. Since $T_{\Lambda}=P_{\Lambda} T J_{\Lambda}$, we have

$$
\Phi(\Lambda) x=U_{\Lambda} P_{\Lambda} T J_{\Lambda} U_{\Lambda}^{-1} x \quad \text { and } \quad \Phi(\Lambda)^{*} x=U_{\Lambda} P_{\Lambda} T^{*} J_{\Lambda} U_{\Lambda}^{-1} x .
$$

By Claim 3.8, the map $\Lambda \mapsto f_{\Lambda}:=T J_{\Lambda} U_{\Lambda}^{-1} x$ is continuous from $\mathcal{K}_{\text {perf }}(E)$ into $L^{2}(0,2 \pi)$. By Claim 3.8 again, and since the map $\Lambda \mapsto U_{\Lambda} P_{\Lambda}$ takes values in a bounded subset of $\mathfrak{B}\left(L^{2}(0,2 \pi), \mathcal{H}\right)$, the map $\Lambda \mapsto \Phi(\Lambda) x=U_{\Lambda} P_{\Lambda} f_{\Lambda}$ is continuous. Likewise, the map $\Lambda \mapsto \Phi(\Lambda)^{*} x$ is continuous. The equality $\Phi^{-1}\left(\mathrm{CH}_{M}(\mathcal{H})\right)=\mathcal{W}$, the continuity of $\Phi$ and Fact 3.6 now imply that $\left.\mathrm{CH}_{M}(\mathcal{H})\right)$ is a true $\Pi_{3}^{0}$ set in $\left(\mathfrak{B}_{M}(\mathcal{H})\right.$, SOT* $)$.

3.1.3. Complexity of $\operatorname{UFHC}_{M}(\mathcal{H})$ and $\operatorname{UFHC}_{M}(\mathcal{H}) \cap \mathrm{CH}_{M}(\mathcal{H})$. - In this subsection, we study the descriptive complexity of the sets $\operatorname{UFHC}_{M}(\mathcal{H})$ and $\operatorname{UFHC}_{M}(\mathcal{H}) \cap \mathrm{CH}_{M}(\mathcal{H})$ in $\left(\mathfrak{B}_{M}(\mathcal{H})\right.$, SOT* $)$, for any $M>1$. Although we have been unable to determine the exact complexity of these sets, we obtain some simple upper bounds and some rather non-trivial lower bounds. Our first result reads as follows.

Proposition 3.9. - For any $M>1$, the set $\mathrm{UFHC}_{M}(\mathcal{H})$ is $\Pi_{4}^{0}$ in $\left(\mathfrak{B}_{M}(\mathcal{H})\right.$, SOT), hence also in $\left(\mathfrak{B}_{M}(\mathcal{H})\right.$, SOT $\left.^{*}\right)$.

Proof. - The proof of Proposition 3.9 relies on the following observation, first made in [9] and then developed in [14].

Fact 3.10. - Let $\left(V_{q}\right)_{q \geq 1}$ be a countable basis of (non-empty) open subsets of $\mathcal{H}$, and let $T$ be a bounded operator on $\mathcal{H}$.

(1) Assume that $T$ belongs to $\operatorname{UFHC}(\mathcal{H})$, and let $x_{0} \in H$ be a $\mathcal{U}$-frequently hypercyclic vector for $T$. For each $q \geq 1$, denote by $2 \delta_{q}$ the upper density of the set $\mathcal{N}_{T}\left(x_{0}, V_{q}\right)$. For any integers $q, N \geq 1$, define

$$
G_{q, N}:=\left\{x \in \mathcal{H} ; \exists n \geq N: \#\left\{1 \leq i \leq n ; T^{i} x \in V_{q}\right\} \geq n \delta_{q}\right\} .
$$

Then

$$
G:=\bigcap_{q \geq 1} \bigcap_{N \geq 1} G_{q, N}
$$


is a dense $G_{\delta}$ subset of $\mathcal{H}$ which consists entirely of $\mathcal{U}$-frequently hypercyclic vectors for $T$.

(2) Conversely, assume that there exists a sequence of positive numbers $\left(\delta_{q}\right)_{q \geq 1}$ such that for any $q, N \geq 1$, the open set $G_{q, N}$ defined by the formula above is dense in $\mathcal{H}$. Then $T$ is $\mathcal{U}$-frequently hypercyclic and admits a dense $G_{\delta}$ set of $\mathcal{U}$-frequently hypercyclic vectors $x$ which satisfy

$$
\overline{\operatorname{dens}} \mathcal{N}_{T}\left(x, V_{q}\right) \geq \delta_{q} \quad \text { for every } q \geq 1 .
$$

Let us denote by $\mathbb{Q}_{+}^{*}$ the set of all positive rational numbers. It follows immediately from Fact 3.10 that an operator $T \in \mathfrak{B}_{M}(\mathcal{H})$ belongs to $\operatorname{UFHC}_{M}(\mathcal{H})$ if and only if

$$
\forall q \geq 1 \exists \delta \in \mathbb{Q}_{+}^{*} \forall N \geq 1 \forall p \geq 1 \exists x \in V_{p} \exists n \geq N: \#\left\{1 \leq i \leq n ; T^{i} x \in V_{q}\right\} \geq n \delta .
$$

In order to prove that $\operatorname{UFHC}_{M}(\mathcal{H})$ is $\Pi_{4}^{0}$ in $\left(\mathfrak{B}_{M}(\mathcal{H}), \mathrm{SOT}\right)$, it is enough to check that each set

$$
\left\{T \in \mathfrak{B}_{M}(\mathcal{H}) ; \#\left\{1 \leq i \leq n ; T^{i} x \in V_{q}\right\} \geq n \delta\right\}
$$

is SOT-open in $\mathfrak{B}_{M}(\mathcal{H})$. This is easy: if $T_{0}$ belong to this set, one can find $k \geq n \delta$ distinct integers $1 \leq i_{1}, \ldots i_{k} \leq n$ such that $T_{0}^{i_{s}} x$ belongs to $V_{q}$ for every $s=1, \ldots, k$. If $T \in \mathfrak{B}_{M}(\mathcal{H})$ is sufficiently close to $T_{0}$ for the SOT-topology, it still satisfies $T^{i_{s}} x \in V_{q}$ for every $1 \leq s \leq k$, and thus belongs to our set. Hence $\operatorname{UFHC}_{M}(\mathcal{H})$ is $\Pi_{4}^{0}$ in $\left(\mathfrak{B}_{M}(\mathcal{H})\right.$, SOT), and thus in $\left(\mathfrak{B}_{M}(\mathcal{H})\right.$, SOT$\left.^{*}\right)$ as well.

Our next result deals with the complexity of the class $\mathrm{UFHC}_{M}(\mathcal{H}) \cap \mathrm{CH}_{M}(\mathcal{H})$.

Proposition 3.11. - The set $\mathrm{UFHC}_{M}(\mathcal{H}) \cap \mathrm{CH}_{M}(\mathcal{H})$ is a difference of $\boldsymbol{\Sigma}_{3}^{0}$ sets in $\left(\mathfrak{B}_{M}(\mathcal{H})\right.$, $\left.\mathrm{SOT}^{*}\right)$, i.e. it can be written as $A \backslash B$, where $A$ and $B$ are both $\mathrm{SOT}^{*}-\Sigma_{3}^{0}$ sets. Besides, $\mathrm{UFHC}_{M}(\mathcal{H}) \cap \mathrm{CH}_{M}(\mathcal{H})$ is neither $\mathrm{SOT}^{*}-\boldsymbol{\Sigma}_{3}^{0}$, nor $\mathrm{SOT}^{*}-\Pi_{3}^{0}$ in $\mathfrak{B}_{M}(\mathcal{H})$. More pre-

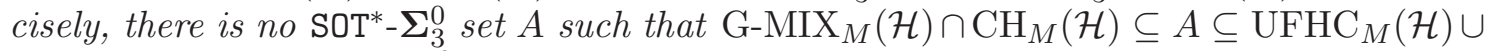
$\mathrm{CH}_{M}(\mathcal{H})$, and no $\mathrm{SOT}^{*}-\Pi_{3}^{0}$ set $B$ such that $\operatorname{FHC}_{M}(\mathcal{H}) \cap \mathrm{CH}_{M}(\mathcal{H}) \subseteq B \subseteq \operatorname{UFHC}_{M}(\mathcal{H})$.

As a straightforward consequence of Proposition 3.11, we obtain:

Corollary 3.12. - The set $\mathrm{UFHC}_{M}(\mathcal{H})$ is neither $\boldsymbol{\Sigma}_{3}^{0}$, nor $\boldsymbol{\Pi}_{3}^{0}$ in $\left(\mathfrak{B}_{M}(\mathcal{H}), \mathrm{SOT}^{*}\right)$.

Proof. - By Proposition 3.11, $\operatorname{UFHC}_{M}(\mathcal{H})$ is not $\boldsymbol{\Sigma}_{3}^{0}$, and it is not $\boldsymbol{\Pi}_{3}^{0}$ because $\mathrm{CH}_{M}(\mathcal{H})$ is $\Pi_{3}^{0}$ and $\mathrm{UFHC}_{M}(\mathcal{H}) \cap \mathrm{CH}_{M}(\mathcal{H})$ is not.

Another immediate consequence is

Corollary 3.13. - The set $\operatorname{TMIX}_{M}(\mathcal{H}) \cap \mathrm{CH}_{M}(\mathcal{H})$ is a true $\Pi_{3}^{0}$ set in $\left(\mathfrak{B}_{M}(\mathcal{H}), \mathrm{SOT}^{*}\right)$.

Proof. - By Propositions 3.2 and 3.4. $\operatorname{TMIX}_{M}(\mathcal{H}) \cap \mathrm{CH}_{M}(\mathcal{H})$ is $\Pi_{3}^{0}$; and by Proposition 3.11, it is not $\Sigma_{3}^{0}$.

Proof of Proposition 3.11. - The proof of Proposition 3.11 relies on the following fact, which characterizes in a rather surprising way the $\mathcal{U}$-frequently hypercyclic operators within the class of chaotic operators. (We will come back to this at the end of Section 5 ).

Fact 3.14. - Let $T$ be a chaotic operator on $\mathcal{H}$. Then, $T$ is $\mathcal{U}$-frequently hypercyclic if and only if $c(T)>0$.

Proof of Fact 3.14 - This follows from Theorem 5.23, to be proved below. 
We now start the proof of Proposition 3.11, which we divide into three parts.

Part 1. $\mathrm{UFCH}_{M}(\mathcal{H}) \cap \mathrm{CH}_{M}(\mathcal{H})$ is a difference of $\mathrm{SOT}^{*}-\Sigma_{3}^{0}$ subsets of $\mathfrak{B}_{M}(\mathcal{H})$.

By Fact 3.14, we have $\mathrm{UFCH}_{M}(\mathcal{H}) \cap \mathrm{CH}_{M}(\mathcal{H})=\mathrm{c}_{M}^{+}(\mathcal{H}) \cap \mathrm{CH}_{M}(\mathcal{H})$, where

$$
\mathrm{c}^{+}(\mathcal{H})=\{T \in \mathrm{HC}(\mathcal{H}) ; c(T)>0\} .
$$

Hence, since $\mathrm{CH}_{M}(\mathcal{H})$ is $\mathrm{SOT}^{*}-\Pi_{3}^{0}$ by Proposition [3.4, it suffices to show that $\mathrm{c}_{M}^{+}(\mathcal{H})$ is SOT* $-\boldsymbol{\Sigma}_{3}^{0}$. Let $\left(V_{q}\right)_{q \geq 1}$ be a countable basis of non-empty open subsets of $\mathcal{H}$, and observe that, by the definition of $c(T)$, an operator $T \in \mathrm{HC}_{M}(\mathcal{H})$ belongs to $\mathrm{c}^{+}(\mathcal{H})$ if and only if

$$
\exists \varepsilon \in \mathbb{Q}^{+*} \forall q \geq 1 \forall N \geq 1 \exists x \in V_{q} \exists n \geq N: \quad \#\left\{1 \leq i \leq n ;\left\|T^{i} x\right\|<1\right\} \geq n \varepsilon .
$$

This condition is easily seen to define an $\operatorname{SOT}^{*}-\Sigma_{3}^{0}$ subset of $\mathfrak{B}_{M}(\mathcal{H})$; and since $\operatorname{HC}_{M}(\mathcal{H})$ is $G_{\delta}$ in $\mathfrak{B}_{M}(\mathcal{H})$, it follows that $\mathrm{c}_{M}^{+}(\mathcal{H})$ is $\mathrm{SOT}^{*}-\Sigma_{3}^{0}$.

Part 2. There is no $\mathrm{SOT}^{*}-\boldsymbol{\Sigma}_{3}^{0}$ subset $A$ of $\mathfrak{B}_{M}(\mathcal{H})$ such that $\mathrm{G}-\mathrm{MIX}_{M}(\mathcal{H}) \cap \mathrm{CH}_{M}(\mathcal{H}) \subseteq$ $A \subseteq \mathrm{UFHC}_{M}(\mathcal{H}) \cup \mathrm{CH}_{M}(\mathcal{H})$.

In order to prove this, we need some preliminary facts. Let us choose a sequence $\left(\mathcal{H}_{n}\right)_{n \geq 1}$ of infinite-dimensional closed subspaces of $\mathcal{H}$ such that $\mathcal{H}$ can be decomposed as the orthogonal direct sum of the spaces $\mathcal{H}_{n}$, i.e. $\mathcal{H}=\bigoplus_{n \geq 1} \mathcal{H}_{n}$.

Fact 3.15. - Let $T \in \mathfrak{B}(\mathcal{H})$ have the form $T=\bigoplus_{n \geq 1} T_{n}$ with respect to the decomposition $\mathcal{H}=\bigoplus_{n \geq 1} \mathcal{H}_{n}$, where $T_{n} \in \mathfrak{B}\left(\mathcal{H}_{n}\right)$ for each $n \geq 1$.

(i) If all operators $T_{n}$ are chaotic and mixing in the Gaussian sense, then so is $T$.

(ii) If $T$ is $\mathcal{U}$-frequently hypercyclic or chaotic, then so are all operators $T_{n}$.

Proof of Fact 3.15, - (i) Assume that all operators $T_{n}$ are chaotic and mixing in the Gaussian sense, and fix for each $n \geq 1$ a mixing Gaussian measure with full support $\mu_{n}$ for $T_{n}$. Each second-order moment $\int_{\mathcal{H}_{n}}\left\|x_{n}\right\|^{2} d \mu\left(\left(x_{n}\right)\right)$ is finite; and by rescaling (i.e. replacing each measure $\mu_{n}$ by a measure $\tilde{\mu}_{n}$ defined by setting $\tilde{\mu}_{n}(B)=\mu_{n}\left(\varepsilon_{n}^{-1} B\right)$ for every Borel subset $B$ of $\mathcal{H}_{n}$, for a suitably small $\varepsilon_{n}>0$ ), we may assume without loss of generality that the series $\sum_{n \geq 1} \int_{\mathcal{H}_{n}}\left\|x_{n}\right\|^{2} d \mu_{n}\left(x_{n}\right)$ is convergent.

Let us denote by $\boldsymbol{\mu}$ the infinite product measure $\bigotimes_{n \geq 1} \mu_{n}$ on the product space $\prod_{n \geq 1} \mathcal{H}_{n}$. Then

$$
\int_{\prod_{n \geq 1} \mathcal{H}_{n}}\left(\sum_{n=1}^{\infty}\left\|x_{n}\right\|^{2}\right) d \boldsymbol{\mu}\left(\left(x_{n}\right)\right)=\sum_{n=1}^{\infty} \int_{\mathcal{H}_{n}}\left\|x_{n}\right\|^{2} d \mu_{n}\left(x_{n}\right)<\infty,
$$

from which it follows that $\boldsymbol{\mu}$ is in fact concentrated on the set

$$
\mathbf{H}:=\left\{\left(x_{n}\right)_{n \geq 1} \in \prod_{n \geq 1} \mathcal{H}_{n} ; \sum_{n=1}^{\infty}\left\|x_{n}\right\|^{2}<\infty\right\} .
$$

We may therefore define a probability measure $\mu$ on $\mathcal{H}$ as follows: for any Borel subset $C$ of $\mathcal{H}$,

$$
\mu(C)=\mu\left(\left\{\left(x_{n}\right)_{n \geq 1} \in \mathbf{H} ; \sum_{n=1}^{\infty} x_{n} \in C\right\}\right) .
$$

This measure $\mu$ is obviously Gaussian, and it is $T$-invariant because each measure $\mu_{n}$ is $T_{n}$-invariant. Indeed, for any bounded Borel function $f: \mathcal{H} \rightarrow \mathbb{R}$, we have

$$
\int_{\mathcal{H}}(f \circ T) d \mu=\int_{\mathbf{H}} f\left(\sum_{n=1}^{\infty} T_{n} x_{n}\right) d \boldsymbol{\mu}\left(\left(x_{n}\right)\right)=\int_{\mathbf{H}} f\left(\sum_{n=1}^{\infty} x_{n}\right) d \boldsymbol{\mu}\left(\left(x_{n}\right)\right)=\int_{\mathcal{H}} f d \mu .
$$


The measure $\mu$ has full support. Let indeed $V$ be a non-empty open set in $\mathcal{H}$. Choose a point $a \in V$, and $\varepsilon>0$ such that $B(a, 2 \varepsilon) \subseteq V$. We may assume that $a$ belongs to $\mathcal{H}_{1} \oplus \cdots \oplus \mathcal{H}_{N}$ for some $N \geq 1$, where $N$ is so large that

$$
\int_{\mathbf{H}^{N}} \sum_{n>N}\left\|x_{n}\right\|^{2} d \boldsymbol{\mu}^{N}\left(\left(x_{n}\right)\right)<\varepsilon^{2},
$$

where $\mathbf{H}^{N}:=\left\{\left(x_{n}\right)_{n \geq N} \in \prod_{n \geq N} \mathcal{H}_{n} ; \sum_{n=1}^{\infty}\left\|x_{n}\right\|^{2}<\infty\right\}$ and $\boldsymbol{\mu}^{N}:=\bigotimes_{n>N} \mu_{n}$. Then the set $\left\{\left(x_{n}\right)_{n \geq 1} \in \mathbf{H} ; \sum_{n \geq 1} x_{n} \in V\right\}$ contains

$$
\mathbf{A}:=\left\{\left(x_{n}\right)_{n \geq 1} \in \mathbf{H} ; x_{1}+\cdots+x_{N} \in B(a, \varepsilon) \text { and } \sum_{n>N}\left\|x_{n}\right\|^{2}<\varepsilon^{2}\right\} .
$$

Moreover, if we set $\mathbf{A}^{N}:=\left\{\left(x_{n}\right)_{n>N} ; \sum_{n>N}\left\|x_{n}\right\|<\varepsilon^{2}\right\}$, then $\boldsymbol{\mu}^{N}\left(\mathbf{A}^{N}\right)>0$ by (6). Also, setting $\boldsymbol{\mu}_{N}:=\bigotimes_{n \leq N} \mu_{n}$ and $\mathbf{A}_{N}:=\left\{\left(x_{1}, \ldots, x_{N}\right) ; x_{1}+\cdots+x_{N} \in B(a, \varepsilon)\right\}$, the fact that the measures $\mu_{1}, \ldots, \mu_{N}$ have full support implies that $\boldsymbol{\mu}_{N}\left(\mathbf{A}_{N}\right)>0$. Hence $\mu(V) \geq \mu(A)=\boldsymbol{\mu}_{N}\left(\mathbf{A}_{N}\right) \boldsymbol{\mu}^{N}\left(\mathbf{A}^{N}\right)>0$.

Finally, $T$ is mixing with respect to $\mu$ because each operator $T_{n}, n \geq 1$, is mixing with respect to $\mu_{n}$ : this can be shown easily by checking that $\mu\left(A \cap T^{-k}(B)\right) \rightarrow \mu(A) \mu(B)$ as $k \rightarrow \infty$ for any Borel sets $A, B \subseteq \mathcal{H}$ whose definition depends on finitely many coordinates only with respect to the decomposition $\mathcal{H}=\bigoplus_{n \geq 1} \mathcal{H}_{n}$.

Thus, we have shown that $T$ is mixing in the Gaussian sense. Finally, since all operators $T_{n}$ are chaotic, it is easily checked that the periodic points of $T$ are dense in $\mathcal{H}$; hence $T$ is chaotic.

(ii) Fix $n \geq 1$. If we denote by $\pi_{n}: \mathcal{H} \rightarrow \mathcal{H}_{n}$ the canonical projection of $\mathcal{H}$ onto $\mathcal{H}_{n}$, then $T_{n} \pi_{n}=\pi_{n} T$. Since $\pi_{n}$ is continuous and onto (that it has dense range would suffice for our argument), (ii) follows easily: if $x \in \mathcal{H}$ is a $\mathcal{U}$-frequently hypercyclic vector for $T$, then $x_{n}:=\pi_{n} x$ is a $\mathcal{U}$-frequently hypercyclic vector for $T_{n}$; and if $T$ is chaotic, then $\pi_{n}(\mathrm{HC}(T)) \subseteq \mathrm{HC}\left(T_{n}\right)$ and $\pi_{n}(\operatorname{Per}(T))$ is a dense subset of $\mathcal{H}_{n}$ consisting of periodic vectors for $T_{n}$.

Recall that we denote by $\mathbf{Q}$ the set of all "rationals" of the Cantor space $\mathbf{C}$,

$$
\mathbf{Q}=\left\{\alpha=(\alpha(i))_{i \geq 1} \in \mathbf{C} ; \alpha(i)=0 \text { for all but finitely many } i\right\} .
$$

Fact 3.16. - There exists a continuous map $\alpha \mapsto T_{\alpha}$ from $\mathbf{C}$ into $\left(\mathfrak{B}_{M}(\mathcal{H}), \mathrm{SOT}^{*}\right)$ such that the following holds true:

- if $\alpha \in \mathbf{Q}$, then $T_{\alpha}$ is chaotic and mixing in the Gaussian sense, and hence chaotic and $\mathcal{U}$-frequently hypercyclic;

- if $\alpha \notin \mathbf{Q}$, then the spectrum of $T_{\alpha}$ is reduced to the point $\{1\}$, and hence $T_{\alpha}$ is not chaotic and not $\mathcal{U}$-frequently hypercyclic.

Proof of Fact 3.16. - The very last part of the second assertion follows from the fact that the spectrum of a chaotic or $\mathcal{U}$-frequently hypercyclic operator has no isolated points $(48)$.

We assume that $\mathcal{H}=\ell^{2}(\mathbb{N})$, endowed with its canonical basis. For any unilateral weight sequence $\boldsymbol{\omega}=\left(\omega_{j}\right)_{j \geq 1}$, let us denote as usual by $B_{\boldsymbol{\omega}}$ the associated weighted shift acting on $\mathcal{H}$. If $\inf _{j \geq 1}\left|\omega_{j}\right|>0$, the operator $T_{\omega}=I+B_{\omega}$ is chaotic and mixing in the Gaussian sense since it admits a spanning holomorphic eigenvector field defined in a neighborhood of the point 1 (see Fact 2.8). On the other hand, if $\omega_{j} \rightarrow 0$ as $j \rightarrow \infty$, then $\sigma\left(B_{\boldsymbol{\omega}}\right)=\{0\}$, 
so that $\sigma\left(T_{\boldsymbol{\omega}}\right)=\{1\}$. So, setting $c:=M-1>0$, it is enough to find a continuous map $\alpha \mapsto \boldsymbol{\omega}(\alpha)$ from $\mathbf{C}$ into $(0, c]^{\mathbb{N}}$ such that $\inf _{j \geq 1} \omega_{j}(\alpha)>0$ if $\alpha \in \mathbf{Q}$ while $\omega_{j}(\alpha) \rightarrow 0$ as $j \rightarrow \infty$ if $\alpha \notin \mathbf{Q}$. Once this is done, the map $\alpha \mapsto T_{\alpha}:=T_{\boldsymbol{\omega}(\alpha)}$ will enjoy the two properties stated in Fact 3.16. Now, it is quite easy to define such a map $\alpha \mapsto \boldsymbol{\omega}(\alpha)$ : just set, for every $j \geq 1$,

$$
\omega_{j}(\alpha):=\frac{c}{1+n_{j}(\alpha)}, \quad \text { where } \quad n_{j}(\alpha)=\#\{i \leq j ; \alpha(i)=1\} .
$$

Fact 3.16 is thus proved.

It is now easy to deduce from Facts 3.15 and 3.16 that no subset $A$ of $\mathfrak{B}_{M}(\mathcal{H})$ with the property that $\mathrm{G}_{-\mathrm{MIX}_{M}}(\mathcal{H}) \cap \mathrm{CH}_{M}(\mathcal{H}) \subseteq A \subseteq \operatorname{UFHC}_{M}(\mathcal{H}) \cup \mathrm{CH}_{M}(\mathcal{H})$ can be $\mathrm{SOT}^{*}-\Sigma_{3}^{0}$. Let us fix such a set $A$. Decompose $\mathcal{H}$ as $\mathcal{H}=\bigoplus_{n \geq 1} \mathcal{H}_{n}$, where the spaces $\mathcal{H}_{n}, n \geq 1$, are infinite-dimensional. Let us define a map $\Phi: \mathbf{C}^{\mathbb{N}} \rightarrow \mathfrak{B}_{M}(\mathcal{H})$ as follows: for every $\bar{\alpha}=\left(\alpha_{n}\right)_{n \geq 1} \in \mathbf{C}^{\mathbb{N}}$,

$$
\Phi(\bar{\alpha}):=\bigoplus_{n \geq 1} T_{\alpha_{n}}
$$

where the operators $T_{\alpha_{n}} \in \mathfrak{B}_{M}\left(\mathcal{H}_{n}\right), n \geq 1$, are given by Fact 3.16. Then $\Phi$ is continuous, and Facts 3.15 and 3.16 imply that

$$
\Phi^{-1}(A)=W=\left\{\bar{\alpha}=\left(\alpha_{n}\right)_{n \geq 1} \in \mathbf{C}^{\mathbb{N}} ; \forall n \geq 1: \alpha_{n} \in \mathbf{Q}\right\} .
$$

This concludes the proof since $W$ is a true $\boldsymbol{\Pi}_{3}^{0}$ subset of $\mathbf{C}^{\mathbb{N}}$, hence not a $\boldsymbol{\Sigma}_{3}^{0}$ set.

Part 3. There is no SOT*- $\Pi_{3}^{0}$ set $B$ such that $\mathrm{FHC}_{M}(\mathcal{H}) \cap \mathrm{CH}_{M}(\mathcal{H}) \subseteq B \subseteq \operatorname{UFHC}_{M}(\mathcal{H})$.

In order to prove this, we will make use of the machinery developed in Section 6 below. The reader may therefore prefer to skip the proof and return to it after reading Section 6 ,

In what follows, we assume that $\mathcal{H}=\ell_{2}(\mathbb{N})$, endowed with its canonical basis. Let $M_{0}$ be such that $1<M_{0}<M$, and choose an even integer $C \geq 3$ large enough to have

$$
M_{0}+\sum_{k=1}^{\infty} 2^{k-1} M_{0}^{-\frac{1}{2} C^{k}} \leq M \quad \text { and } \quad \sum_{k=1}^{\infty} 2^{k} M_{0}^{-\frac{1}{12} C^{k}} C^{k} \leq 1 .
$$

Now, let us denote by $\mathcal{D}$ the set of all infinite sequence of integers $\delta=\left(\delta^{(k)}\right)_{k \geq 0}$ with $\delta^{(0)}=1$ such that $C \leq \delta^{(k)} \leq C^{4 k}$ and $\delta^{(k)} \geq C \delta^{(k-1)}$ for every $k \geq 1$. This is a closed subset of $\mathbb{N}^{\mathbb{N}}$ and hence a Polish space. For any element $\delta$ of $\mathcal{D}$, we denote by $T_{\delta}$ the operator of $\mathrm{C}_{+}$-type on $\mathcal{H}$ defined as follows: for any $k \geq 1$

$$
\Delta^{(k)}=C^{4 k}, \quad v^{(k)}=M_{0}^{-\frac{1}{2} \delta^{(k)}} \quad \text { and } \quad w_{i}^{(k)}=\left\{\begin{array}{ccc}
M_{0} & \text { if } & 1 \leq i \leq \delta^{(k)}, \\
1 & \text { if } & \delta^{(k)}<i<\Delta^{(k)} .
\end{array}\right.
$$

Note that in the terminology of Section 6. $T_{\delta}$ looks exactly like an operator of $C_{+, 1^{-}}$ type with $\tau^{(k)}=\frac{1}{2} \delta^{(k)}$, except that in the definition of the weights $v^{(k)}$ and $w_{i}^{(k)}$ the constant 2 has been replaced by $M_{0}$. (See Subsection 6.2 for the definition of $\mathrm{C}_{+}$-type operators, and Subsection 6.5 for the definition of $\mathrm{C}_{+, 1}$-type operators.) Observe that if $\delta=\left(\delta^{(k)}\right)_{k \geq 0}$ belongs to $\mathcal{D}$, then $\delta^{(k)} \geq C^{k}$ for all $k \geq 1$. By (7), it follows that $\left\|T_{\delta}\right\| \leq M$ for every $\delta \in \mathcal{D}$. Moreover, it is clear that the map $\delta \mapsto T_{\delta}$ is continuous from $\mathcal{D}$ into $\left(\mathfrak{B}_{M}(\mathcal{H})\right.$, SOT $\left.^{*}\right)$.

The key point of the proof that $\mathrm{UFHC}_{M}(\mathcal{H}) \cap \mathrm{CH}_{M}(\mathcal{H})$ is not SOT*- $\Pi_{3}^{0}$ is the following fact, which is actually nothing but a reformulation of Theorem 6.18 in a slightly different setting. 
Fact 3.17. - Let $\delta=\left(\delta^{(k)}\right)_{k>0}$ belong to $\mathcal{D}$. Then the operator $T_{\delta}$ is always chaotic, and $T_{\delta}$ is $\mathcal{U}$-frequently hypercyclic if and only if $\delta^{(k)} / C^{4 k}$ does not tend to 0 as $k \rightarrow \infty$; in which case $T_{\delta}$ is in fact frequently hypercyclic.

Proof of Fact 3.17 - We apply a modified version of Theorem 6.18, with $p=2$. Accordingly, we set for every $k \geq 1$

$$
\gamma_{k}:=M_{0}^{\delta^{(k-1)}-\frac{1}{2} \delta^{(k)}} \sqrt{\Delta^{(k)}}=M_{0}^{\delta^{(k-1)}-\frac{1}{2} \delta^{(k)}} C^{2 k} .
$$

Since $\delta^{(k)} \leq C^{-1} \delta^{(k+1)} \leq \frac{1}{3} \delta^{(k+1)}$, we have $\delta^{(k)}-\frac{1}{2} \delta^{(k+1)} \leq-\frac{1}{2} \delta^{(k)} \leq \delta^{(k-1)}-\frac{1}{2} \delta^{(k)}$, from which it follows easily that the sequence $\left(\gamma_{k}\right)_{k \geq 1}$ is non-increasing. Moreover, we have $\gamma_{k} \leq M_{0}^{-\frac{1}{6} C^{k}} C^{2 k}$ for all $k \geq 1$. Hence condition (77) above yields that

$$
\sum_{k=1}^{\infty} 2^{k} \gamma_{k}^{1 / 2} \leq 1
$$

The analogues of the "additional assumptions" in Theorem 6.18 are thus satisfied, and it follows that $T_{\delta}$ is $\mathcal{U}$-frequently hypercyclic on $\mathcal{H}$ if and only if $\delta^{(k)} / C^{4 k}$ does not tend to 0 as $k \rightarrow \infty$.

Now, let $B \subseteq \mathfrak{B}_{M}(\mathcal{H})$ have the property that $\operatorname{FHC}_{M}(\mathcal{H}) \cap \mathrm{CH}_{M}(\mathcal{H}) \subseteq B \subseteq \mathrm{UFHC}_{M}(\mathcal{H})$. Consider the map $\Phi: \mathcal{D} \rightarrow \mathfrak{B}_{M}(\mathcal{H})$ defined by setting $\Phi(\delta):=T_{\delta}$ for every $\delta \in \mathcal{D}$. As already mentioned, the map $\Phi$ is continuous from $\mathcal{D}$ into $\left(\mathfrak{B}_{M}(\mathcal{H}), \mathrm{SOT}^{*}\right)$. By Fact 3.17 we have

$$
\Phi^{-1}(B)=\mathcal{D} \backslash \mathcal{D}_{0}, \quad \text { where } \quad \mathcal{D}_{0}=\left\{\delta \in \mathcal{D} ; C^{-4 k} \delta^{(k)} \rightarrow 0 \text { as } k \rightarrow \infty\right\} .
$$

Now, a proof quite similar to that of Fact 3.3 shows that $\mathcal{D}_{0}$ is a true $\Pi_{3}^{0}$ set in $\mathcal{D}$, so that $\mathcal{D} \backslash \mathcal{D}_{0}$ is a true $\boldsymbol{\Sigma}_{3}^{0}$ set in $\mathcal{D}$. Hence $B$ cannot be $\boldsymbol{\Pi}_{3}^{0}$ in $\left(\mathfrak{B}_{M}(\mathcal{H})\right.$, $\left.\mathrm{SOT}^{*}\right)$.

Remark 3.18. - Propositions 3.4 and 3.11 together formally yield the statement that, since $\operatorname{UFHC}_{M}(\mathcal{H}) \cap \mathrm{CH}_{M}(\mathcal{H})$ is not $\Pi_{3}^{0}$ while $\mathrm{CH}_{M}(\mathcal{H})$ is $\Pi_{3}^{0}$ in $\left(\mathfrak{B}_{M}(\mathcal{H})\right.$, SOT* ${ }^{*}$, the class $\mathrm{CH}_{M}(\mathcal{H}) \backslash \mathrm{UFHC}_{M}(\mathcal{H})$ is not void, i.e. there exist chaotic operators in $\mathfrak{B}_{M}(\mathcal{H})$ which are not $\mathcal{U}$-frequently hypercyclic. However, there is nothing magic here: the proof of Proposition 3.11 relies heavily on a construction, carried out in Section 6, of explicit chaotic operators which are not $\mathcal{U}$-frequently hypercyclic.

Remark 3.19. - The proof of Proposition 3.11 has established that the $\operatorname{set}_{\mathrm{M}}^{+}(\mathcal{H})=$ $\left\{T \in \mathrm{HC}_{M}(\mathcal{H}) ; c(T)>0\right\}$ is a true $\mathrm{SOT}^{*}-\boldsymbol{\Sigma}_{3}^{0}$ subset of $\mathfrak{B}_{M}(\mathcal{H})$.

Remark 3.20. - An "alternative" proof of the Borelness of $\mathrm{UFHC}_{M}(\mathcal{H})$ could run as follows. According to Fact 3.10, an operator $T$ belongs to $\operatorname{UFHC}(\mathcal{H})$ if and only if quasi-all vectors $x \in H$ (in the Baire category sense) are $\mathcal{U}$-frequently hypercyclic for $T$. So, for $T \in \mathfrak{B}_{M}(\mathcal{H})$, we may write

$$
T \in \mathrm{UFHC}_{M}(\mathcal{H}) \Longleftrightarrow \forall^{*} x \in \mathcal{H}(x \text { is } \mathcal{U} \text {-frequently hypercyclic for } T) .
$$

Since the relation $B(T, x) \longleftrightarrow(x$ is $\mathcal{U}$-frequently hypercyclic for $T)$ is Borel in the product space $\left(\mathfrak{B}_{M}(\mathcal{H}), \mathrm{SOT}\right) \times \mathcal{H}$, and since the category quantifier $\forall^{*}$ preserves Borelness (see for instance [37, Section 16.A]), it follows that $\operatorname{UFHC}_{M}(\mathcal{H})$ is Borel in $\left(\mathfrak{B}_{M}(\mathcal{H})\right.$, SOT).

Remark 3.21. - It is likely that $\operatorname{TMIX}_{M}(\mathcal{H})$ and $\mathrm{CH}_{M}(\mathcal{H})$ are both true $\boldsymbol{\Pi}_{4}^{0}$ subsets of $\left(\mathfrak{B}_{M}(\mathcal{H})\right.$, SOT $)$, i.e. $\boldsymbol{\Pi}_{4}^{0}$ but not $\boldsymbol{\Sigma}_{4}^{0}$, that $\operatorname{UFHC}_{M}(\mathcal{H})$ is a true $\boldsymbol{\Pi}_{4}^{0}$ set in $\left(\mathfrak{B}_{M}(\mathcal{H})\right.$, SOT $\left.^{*}\right)$, and that $\mathrm{UFHC}_{M}(\mathcal{H}) \cap \mathrm{CH}_{M}(\mathcal{H})$ is a true difference of $\boldsymbol{\Sigma}_{3}^{0}$ sets in $\left(\mathfrak{B}_{M}(\mathcal{H})\right.$, $\left.\mathrm{SOT}^{*}\right)$. However, we have been unable to prove any of these facts. 
Remark 3.22. - In view of Proposition 3.9, it is natural to wonder whether the class of frequently hypercyclic operators $\mathrm{FHC}_{M}(\mathcal{H})$ is also Borel in $\left(\mathrm{HC}_{M}(\mathcal{H})\right.$, SOT). Let $\left(V_{q}\right)_{q \geq 1}$ be a countable basis of open sets for $\mathcal{H}$. An operator $T \in \mathfrak{B}_{M}(\mathcal{H})$ is frequently hypercyclic if and only if

$$
\exists x \in \mathcal{H}\left(\forall q \geq 1 \exists \delta \in \mathbb{Q}_{+}^{*} \exists N \geq 1 \forall n \geq N: \#\left\{1 \leq i \leq n ; T^{i} x \in V_{q}\right\}>n \delta\right) ;
$$

and since the relation $R(T, x)$ defined by the expression between brackets is Borel in $\mathfrak{B}_{M}(\mathcal{H}) \times \mathcal{H}$, we deduce that $\mathrm{FHC}_{M}(\mathcal{H})$ is a $\boldsymbol{\Sigma}_{1}^{1}$ (aka analytic) subset of $\left(\mathfrak{B}_{M}(\mathcal{H})\right.$, SOT). It is quite tempting to conjecture that it is in fact non-Borel in $\left(\mathfrak{B}_{M}(\mathcal{H}), \mathrm{SOT}\right)$.

3.2. Some non-Borel sets in $\mathfrak{B}_{M}(\mathcal{H})$. - We conclude this section by showing that some natural classes of operators defined by dynamical properties are non-Borel in the space $\left(\mathfrak{B}_{M}(\mathcal{H})\right.$, SOT $\left.^{*}\right)$, for any $M>1$.

If $\Gamma$ is any class of Hilbert space operators, we will say that an operator $T$ has a restriction in $\Gamma$ if there is a closed $T$-invariant (infinite-dimensional) subspace $\mathcal{E}$ of $\mathcal{H}$ such that the operator $T_{\mid \mathcal{E}}$ induced by $T$ on $\mathcal{E}$ belongs to $\Gamma(\mathcal{E})$. We denote by $\widehat{\Gamma}(\mathcal{H})$ the class of all operators $T \in \mathfrak{B}(\mathcal{H})$ admitting a restriction in $\Gamma$.

Proposition 3.23. - Let $\Gamma(\mathcal{H})$ be a class of operators on $\mathcal{H}$ such that G-ERG $(\mathcal{H}) \subseteq$ $\Gamma(\mathcal{H}) \subseteq \mathrm{HC}(\mathcal{H})$. For any $M>1$, the set $\widehat{\Gamma}_{M}(\mathcal{H})$ is non-Borel in $\left(\mathfrak{B}_{M}(\mathcal{H})\right.$, $\left.\mathrm{SOT}^{*}\right)$.

Proof. - Recall that for every compact subset $\Lambda$ of $\mathbb{\mathbb { V }} \backslash\{1\}, T_{\Lambda}: \mathcal{H}_{\Lambda} \rightarrow \mathcal{H}_{\Lambda}$ denotes the Kalisch operator associated to $\Lambda$ (this notation has been introduced in the proof of Proposition 3.4). For any compact subset $E$ of $\mathbb{T} \backslash\{1\}$, we denote by $\mathcal{K}_{\infty}(E)$ the set of all infinite compact subsets of $E$.

Fact 3.24. - Let $E$ be a compact subset of $\mathbb{T} \backslash\{1\}$. There exists a sequence $\left(e_{n}\right)_{n \geq 1}$ of Borel maps from $\mathcal{K}_{\infty}(E)$ into $L^{2}(0,2 \pi)$ such that $\left(e_{n}(\Lambda)\right)_{n \geq 1}$ is an orthonormal basis of $\mathcal{H}_{\Lambda}$ for every $\Lambda \in \mathcal{K}_{\infty}(E)$.

Proof of Fact 3.24 - By the Kuratowski-Ryll-Nardzewski Selection Theorem (see [37, Section 12.C]), there exists a sequence $\left(\xi_{n}\right)_{n \geq 1}$ of Borel maps $\xi_{n}: \mathcal{K}(E) \rightarrow E$ such that $\left\{\xi_{n}(\Lambda) ; n \geq 1\right\}$ is a dense subset of $\Lambda$ for every $\Lambda \in \mathcal{K}(E)$. If $\Lambda$ belongs to $\mathcal{K}_{\infty}(E)$, there exists a sequence $\left(\zeta_{n}(\Lambda)\right)_{n \geq 1}$ of distinct elements of $\Lambda$ such that $\left\{\zeta_{n}(\Lambda) ; n \geq 1\right\}$ is dense in $\Lambda$. Indeed, it suffices to set $\zeta_{1}(\Lambda)=\xi_{1}(\Lambda)$ and, for every $n \geq 1, \zeta_{n}(\Lambda)=\xi_{k_{n}}(\Lambda)$ where $k_{n}:=\min \left\{j \geq n ;\right.$ for all $\left.1 \leq i<j, \xi_{i}(\Lambda) \neq \xi_{j}(\Lambda)\right\}$. Then the maps $\Lambda \mapsto \zeta_{n}(\Lambda), n \geq 1$, are Borel on $\mathcal{K}_{\infty}(E)$, and by construction the elements $\zeta_{n}(\Lambda), n \geq 1$, are pairwise distinct and form a dense subset of $\Lambda$. Applying the Gram-Schmidt orthonormalization process to the sequence $\left(f_{\zeta_{n}(\Lambda)}\right)_{n \geq 1}$, which is linearly independent, we get the required orthonormal basis $\left(e_{n}(\Lambda)\right)_{n \geq 1}$ of $\mathcal{H}_{\Lambda}$.

The key step in the proof of Proposition 3.23 is the following result of Waterman [52].

Fact 3.25. - If $\Lambda$ is a compact subset of $\mathbb{T} \backslash\{1\}$, the operator $T_{\Lambda}$ admits spectral synthesis, which means that every closed $T_{\Lambda}$-invariant subspace of $\mathcal{H}_{\Lambda}$ is spanned by the eigenvectors belonging to it. In other words, any invariant subspace $\mathcal{E} \subseteq \mathcal{H}_{\Lambda}$ for $T_{\Lambda}$ has the form $\mathcal{E}=\mathcal{H}_{K}$ for some compact set $K \subseteq \Lambda$. 
We are now ready to prove Proposition 3.23, Let us fix a non-trivial arc $E \subseteq \mathbb{T} \backslash\{1\}$ such that $\left\|T_{E}\right\| \leq M$. As in the proof of Proposition 3.4, let $\left(e_{n}\right)_{n \geq 1}$ be an orthonormal basis of $\mathcal{H}$, and for any $\Lambda \in \mathcal{K}_{\infty}(E)$, denote by $U_{\Lambda}: \mathcal{H}_{\Lambda} \rightarrow \mathcal{H}$ the unitary operator sending $e_{n}(\Lambda)$ to $e_{n}$ for all $n \geq 1$, where $e_{n}(\Lambda)$ is given by Fact 3.24. Then define a map $\Phi: \mathcal{K}_{\infty}(E) \rightarrow \mathfrak{B}_{M}(\mathcal{H})$ by setting

$$
\Phi(\Lambda)=U_{\Lambda} T_{\Lambda} U_{\Lambda}^{-1} \quad \text { for every } \Lambda \in \mathcal{K}_{\infty}(E) .
$$

Proceeding as in the proof of Proposition [3.4, and using the fact that the maps $\Lambda \mapsto$ $e_{n}(\Lambda), n \geq 1$, are Borel on $\mathcal{K}_{\infty}(E)$, we deduce that the map $\Phi$ is Borel from $\mathcal{K}_{\infty}(E)$ into $\left(\mathfrak{B}_{M}(\mathcal{H})\right.$, SOT $\left.^{*}\right)$.

If $\Lambda \in \mathcal{K}_{\infty}(E)$ is uncountable, it admits a perfect subset $K$, so the restriction of $T_{\Lambda}$ to the invariant subspace $\mathcal{H}_{K}$ (which is nothing else but $T_{K}$ ) is ergodic in the Gaussian sense. Thus $\Phi(\Lambda)$ belongs to $\widehat{G-E R} G(\mathcal{H}) \subseteq \widehat{\Gamma}(\mathcal{H})$. On the other hand, if $\Lambda$ is countable then, by Fact $3.25, T_{\Lambda}$ has no hypercyclic restriction. Hence $\Phi(\Lambda)$ belongs to $\mathfrak{B}(\mathcal{H}) \backslash \widehat{H C}(\mathcal{H}) \subseteq$ $\mathfrak{B}(\mathcal{H}) \backslash \widehat{\Gamma}(\mathcal{H})$. So we have proved that

$$
\Phi^{-1}\left(\widehat{\Gamma}_{M}(\mathcal{H})\right)=\mathcal{K}_{\infty}(E) \backslash \mathcal{K}_{\omega}(E) .
$$

Since $\mathcal{K}_{\omega}(E)$ is notoriously non-Borel in $\mathcal{K}(E)$ (see [37, Section 27.B]) and hence also in $\mathcal{K}_{\infty}(E)$ because the latter is Borel in $\mathcal{K}(E)$, this concludes the proof.

Remark 3.26. - The same proof shows that $\widehat{\mathrm{G}-\mathrm{MIX}}_{M}(\mathcal{H})$, the family of all operators $T \in \mathfrak{B}_{M}(\mathcal{H})$ admitting a restriction which is mixing in the Gaussian sense, is non-Borel in $\left(\mathfrak{B}_{M}(\mathcal{H})\right.$, SOT* ${ }^{*}$. Instead of working with the class $\mathcal{K}_{\omega}(E)$ of countable subsets of $E$, one has to consider the class $U_{0} \cap \mathcal{K}(E)$ of compact subsets of $E$ which are sets of extended uniqueness, also called $U_{0}$-sets. By definition, a compact set $\Lambda \subseteq \mathbb{T}$ is a $U_{0}$-set if it is negligible for every probability measure $\sigma$ on $\mathbb{\mathbb { T }}$ whose Fourier coefficients vanish at infinity. It follows from the main result of $\left[8\right.$ that if $\Lambda \subseteq \mathbb{T} \backslash\{1\}$ is not a $U_{0}$-set, then the Kalisch operator $T_{\Lambda}$ has a restriction which is mixing in the Gaussian sense, whereas if $\Lambda$ is a $U_{0}$-set, $T_{\Lambda}$ is not mixing in the Gaussian sense. Moreover, it is well-known that the class $U_{0} \cap \mathcal{K}(E)$ is non-Borel in $\mathcal{K}(E)$ for any non-trivial arc $E \subseteq \mathbb{T}$ (see [38, Th. VIII.2.6]). Using these facts, one can prove the aforementioned result in exactly the same way as above: the key identity

$$
\Phi^{-1}\left(\widehat{\Gamma}_{M}(\mathcal{H})\right)=\mathcal{K}_{\infty}(E) \backslash \mathcal{K}_{\omega}
$$

at the end of the proof of Proposition 3.23 is replaced by

$$
\Phi^{-1}\left(\widehat{\Gamma}_{M}(\mathcal{H})\right)=\mathcal{K}_{\infty}(E) \backslash U_{0}
$$

Remark 3.27. - Let $\Gamma_{0}(\mathcal{H})$ be a class of operators of $\mathcal{H}$ with the following two properties:

(i) $\operatorname{G-ERG}(\mathcal{H}) \subseteq \Gamma_{0}(\mathcal{H})$;

(ii) if $\Lambda$ is any countable compact subset of $\mathbb{T} \backslash\{1\}$, the Kalisch operator $T_{\Lambda}$ does not belong to $\Gamma_{0}(\mathcal{H})$.

Exactly the same proof as that of Proposition 3.23 shows that the following statement holds true: if $\Gamma(\mathcal{H})$ is a class of operators on $\mathcal{H}$ such that $\mathrm{G}-\mathrm{ERG}(H) \subseteq \Gamma(\mathcal{H}) \subseteq \Gamma_{0}(\mathcal{H})$ then, for any $M>1$, the set $\widehat{\Gamma}_{M}(\mathcal{H})$ is non-Borel in $\left(\mathfrak{B}_{M}(\mathcal{H})\right.$, SOT* $)$.

Our aim is now to prove the following result: 
Proposition 3.28. - For any $M>1$, the class $\mathrm{DCH}_{M}(\mathcal{H})$ of distributionally chaotic operators in $\mathfrak{B}_{M}(\mathcal{H})$ is non-Borel in $\left(\mathfrak{B}_{M}(\mathcal{H})\right.$, $\left.\mathrm{SOT}^{*}\right)$.

Note that this is in strong contrast with Proposition 2.37, according to which the class of densely distrobutionally chaotic operators in $\mathfrak{B}_{M}(\mathcal{H})$ is $G_{\delta}$.

The proof of Proposition 3.28 relies on the following observation:

Fact 3.29. - An operator $T \in \mathfrak{B}(\mathcal{H})$ is distributionally chaotic if and only if it has a restriction which is densely distributionally chaotic.

Proof of Fact 3.29] - If there exists a closed subspace $\mathcal{E}$ of $\mathcal{H}$ such that $\left.T\right|_{\mathcal{E}}$ is densely distributionally chaotic, $T$ admits in particular a distributionally irregular vector belonging to $\mathcal{E}$, and hence $T$ is distributionally chaotic. Conversely, assume that $T$ is distributionally chaotic, and let $x \in \mathcal{H}$ be a distributionally irregular vector. Let $\mathcal{E}$ be the closed $T$ invariant subspace spanned by the orbit of $x$, and denote by $S$ the operator induced by $T$ on $\mathcal{E}$. As the vector $x \in \mathcal{E}$ has a distributionally unbounded orbit under the action of $S$ (i.e. $\left\|S^{n} x\right\| \rightarrow \infty$ as $n \rightarrow \infty$ along a subset $B$ of $\mathbb{N}$, with $\overline{\operatorname{dens}}(B)=1$ ), $S$ admits by $[\mathbf{1 0}$, Prop. 8] a comeager set of vectors with distributionally unbounded orbit. Also, the orbit of $x$ under the action of $S$ is distributionally near to 0 (i.e. $\left\|S^{n} x\right\| \rightarrow 0$ as $n \rightarrow \infty$ along a subset $A$ of $\mathbb{N}$, with $\overline{\operatorname{dens}}(A)=1$ ). It follows that $\left\|S^{n} y\right\| \rightarrow 0$ as $n \rightarrow \infty$ along $A$ for every vector $y$ belonging to the linear span of the vectors $S^{p} x, p \geq 0$. Hence $S$ admits a dense set of vectors whose orbit is distributionally near to 0. By [10, Prop. 9], it follows that the set of vectors whose orbit under the action of $S$ is distributionally near to 0 is comeager in $\mathcal{E}$. By the Baire Category Theorem, we conclude that $S$ admits a comeager set of distributionally irregular vectors, i.e. $S$ is densely distributionally chaotic.

We will also need the following characterizations of (densely) distributionally chaotic Kalisch operators:

Fact 3.30. - Let $\Lambda$ be a compact subset of $\mathbb{T} \backslash\{1\}$, and let $T_{\Lambda}$ be the associated Kalisch operator on $H_{\Lambda}$.

(1) The operator $T_{\Lambda}$ is densely distributionally chaotic if and only if $\Lambda$ is a perfect set.

(2) The operator $T_{\Lambda}$ is distributionally chaotic if and only if $\Lambda$ is uncountable.

Proof of Fact 3.30 - Let us start by proving assertion (1). If $\Lambda$ is a perfect set then $T_{\Lambda}$ is an ergodic operator, so that it is in particular densely distributionally chaotic by [29]. Conversely, suppose that $\Lambda$ is not a perfect set, and let $\lambda_{0}$ be an isolated point of $\Lambda$. Then the eigenfunction $f_{\lambda_{0}}$ does not belong to $\mathcal{H}_{\Lambda \backslash\left\{\lambda_{0}\right\}}$, and $\mathcal{H}_{\Lambda}$ can be written as a topological direct $\operatorname{sum} \mathcal{H}_{\Lambda}=\operatorname{span}\left[f_{\lambda_{0}}\right] \oplus \mathcal{H}_{\Lambda \backslash\left\{\lambda_{0}\right\}}$. Hence, there exists a non-zero vector $g_{0}$ of $\mathcal{H}_{\Lambda}$ with $\left\langle g_{0}, f_{\lambda_{0}}\right\rangle=1$ and $g \perp \mathcal{H}_{\Lambda \backslash\left\{\lambda_{0}\right\}}$, and a bounded projection $Q$ of $\mathcal{H}_{\Lambda}$ onto $\mathcal{H}_{\Lambda \backslash\left\{\lambda_{0}\right\}}$, such that $f=\left\langle g_{0}, f\right\rangle f_{\lambda_{0}}+Q f$ for every $f \in \mathcal{H}_{\Lambda}$. It follows that $T_{\Lambda}^{n} f=\left\langle g_{0}, f\right\rangle \lambda_{0}^{n} f_{\lambda_{0}}+T_{\Lambda}^{n} Q f$ for every $n \geq 1$, so that $\left\langle g_{0}, T_{\Lambda}^{n} f\right\rangle=\left\langle g_{0}, f\right\rangle \lambda_{0}^{n}$. Hence $\left\|T_{\Lambda}^{n} f\right\| \geq\left|\left\langle g_{0}, f\right\rangle\right| /\left\|g_{0}\right\|$ for every $n \geq 1$. Thus, we see that the orbit under $T_{\Lambda}$ of any vector $f \in \mathcal{H}_{\Lambda}$ with $\left\langle g_{0}, f\right\rangle \neq 0$ is bounded away from 0 , and hence that no such vector can be distributionally irregular. So $T_{\Lambda}$ is not densely distributionally chaotic.

The proof of assertion (2) relies on Facts 3.25 and 3.29 above. By Fact $3.29, T_{\Lambda}$ is distributionally chaotic if and only if it admits a restriction which is densely distributionally chaotic. Since, by Fact 3.25, any such restriction is of the form $T_{K} \in \mathfrak{B}\left(\mathcal{H}_{K}\right)$, where $K$ is a compact subset of $\Lambda$, it follows from assertion (1) that $T_{\Lambda}$ is distributionally chaotic if 
and only if $\Lambda$ contains a perfect set, which happens exactly when $\Lambda$ is uncountable. This proves (2).

We are now ready for the proof of Proposition 3.28 ,

Proof of Proposition 3.28. - Consider the class $\Gamma(\mathcal{H})=\Gamma_{0}(\mathcal{H}):=\operatorname{DDCH}(\mathcal{H})$. This class $\Gamma_{0}(\mathcal{H})$ satisfies the assumptions of Remark 3.27, G-ERG $(\mathcal{H})$ is contained in $\Gamma_{0}(\mathcal{H})$ by a result of 29], and it follows from Fact 3.30 that whenever $\Lambda$ is a countable subset of $\mathbb{T} \backslash\{1\}$, the Kalisch operator $T_{\Lambda}$ is not (densely) distributionally chaotic. Since G-ERG $(\mathcal{H}) \subseteq \Gamma(\mathcal{H}) \subseteq \Gamma_{0}(\mathcal{H})$, we conclude that for any $M>1, \widehat{\Gamma}_{M}(\mathcal{H})$ is non-Borel in $\left(\mathfrak{B}_{M}(\mathcal{H})\right.$, SOT$\left.^{*}\right)$. But $\widehat{\Gamma}_{M}(\mathcal{H})=\operatorname{DCH}_{M}(\mathcal{H})$ by Fact 3.29, and hence $\operatorname{DCH}_{M}(\mathcal{H})$ is nonBorel in $\left(\mathfrak{B}_{M}(\mathcal{H}), \mathrm{SOT}^{*}\right)$.

Corollary 3.31. - For any $M>1$, the set $\mathrm{DCH}_{M}(\mathcal{H}) \backslash \mathrm{DDCH}_{M}(\mathcal{H})$ of operators in $\mathfrak{B}_{M}(\mathcal{H})$ which are distributionally chaotic but not densely distributionally chaotic is nonBorel in $\left(\mathfrak{B}_{M}(\mathcal{H}), \mathrm{SOT}^{*}\right)$.

Proof. - Since $\operatorname{DDCH}_{M}(H)$ is a $G_{\delta}$ subset of $\left(\mathrm{HC}_{M}(\mathcal{H})\right.$, $\left.\mathrm{SOT}^{*}\right)$ by Proposition 2.37, it is in particular Borel. So one immediately deduces from Proposition 3.28 that $\operatorname{DCH}_{M}(\mathcal{H}) \backslash$ $\operatorname{DDCH}_{M}(\mathcal{H})$ is non-Borel in $\left(\mathfrak{B}_{M}(\mathcal{H})\right.$, $\left.\mathrm{SOT}^{*}\right)$.

Remark 3.32. - Corollary 3.31 formally yields the existence of distributionally chaotic operators which are not densely distributionally chaotic. But the existence of such operators can of course be deduced directly from Proposition 3.30 a Kalisch operator $T_{\Lambda}$ is distributionally chaotic but not densely distributionally chaotic if and only if $\Lambda$ is uncountable but not perfect.

\section{Ergodicity for upper-triangular operators}

4.1. Definitions and setting. - Let us fix an orthonormal basis $\left(e_{k}\right)_{k \geq 1}$ of the complex separable infinite-dimensional Hilbert space $\mathcal{H}$. We will denote by $\mathcal{H}_{00}$ the linear subspace of $\mathcal{H}$ consisting of finitely supported vectors with respect to the basis $\left(e_{k}\right)_{k \geq 1}$.

In this subsection, we concentrate ourselves on the study of the typical properties of operators on $\mathcal{H}$ which are upper-triangular with respect to the basis $\left(e_{k}\right)_{k \geq 1}$, with diagonal coefficients of modulus 1 and pairwise distinct. This class of operators will be denoted by $\mathfrak{T}_{0}(\mathcal{H})$ :

$$
\mathfrak{T}_{0}(\mathcal{H}):=\left\{T \in \mathfrak{B}(\mathcal{H}) ; \forall k \geq 1: T e_{k} \in \operatorname{span}\left[e_{1}, \ldots, e_{k}\right] \text { and }\left|\left\langle T e_{k}, e_{k}\right\rangle\right|=1\right\} .
$$

For each $T \in \mathfrak{T}_{0}(\mathcal{H})$ and $k \geq 1$, we write $\lambda_{k}(T):=\left\langle T e_{k}, e_{k}\right\rangle$. We also define

$$
\mathfrak{T}(\mathcal{H}):=\left\{T \in \mathfrak{T}_{0}(\mathcal{H}) ; \forall j, k \geq 1 \text { with } j \neq k, \lambda_{j}(T) \neq \lambda_{k}(T)\right\}
$$

and

$$
\mathfrak{T}_{\text {ind }}(\mathcal{H}):=\left\{T \in \mathfrak{T}(\mathcal{H}) \text {; the diagonal coefficients } \lambda_{k}(T) \text { are rationally independent }\right\} .
$$

Recall that if $\left(\lambda_{k}\right)_{k \in I}$ is a finite or infinite family of elements of $\mathbb{T}$ with $\lambda_{k}=e^{2 i \pi \theta_{k}}$ for every $k \in I$, it is said to be rationally independent if for any finite subset $F$ of $I$, the family $\left(\theta_{k}\right)_{k \in F}$ consists of $\mathbb{Q}$-independent numbers.

For any $M>0$, we denote by $\mathfrak{T}_{0, M}(\mathcal{H}), \mathfrak{T}_{M}(\mathcal{H})$, and $\mathfrak{T}_{\text {ind, } M}(\mathcal{H})$ the set of operators in $\mathfrak{B}_{M}(\mathcal{H})$ which belong to $\mathfrak{T}_{0}(\mathcal{H}), \mathfrak{T}(\mathcal{H})$, and $\mathfrak{T}_{\text {ind }}(\mathcal{H})$ respectively. It is not difficult to check that these sets are $G_{\delta}$ in $\left(\mathfrak{B}_{M}(\mathcal{H})\right.$, SOT). So we may state: 
Fact 4.1. - For any $M>0$, the sets $\mathfrak{T}_{0, M}(\mathcal{H}), \mathfrak{T}_{M}(\mathcal{H})$, and $\mathfrak{T}_{\text {ind }, M}(\mathcal{H})$ are Polish spaces when endowed with the topology SOT.

Note that (by the Gram-Schmidt orthonormalization process) any bounded operator on $\mathcal{H}$ with spanning unimodular eigenvectors associated to distinct eigenvalues is upper triangular with respect to some orthonormal basis $\left(e_{k}\right)_{k \geq 1}$, and belongs to the associated class $\mathfrak{T}(\mathcal{H})$. Hence, given the importance in linear dynamics of unimodular eigenvectors, it is quite natural to investigate typical properties of elements of the spaces $\left(\mathfrak{T}_{M}(\mathcal{H})\right.$, SOT), $M>1$. Some interesting and unexpected phenomena occur in this setting, which we will describe shortly. Meanwhile, we state for future reference two elementary facts concerning the SOT-continuity of certain maps naturally associated to operators in $\mathfrak{T}(\mathcal{H})$.

When $T$ belongs to $\mathfrak{T}(\mathcal{H})$, there exists for each $k \geq 1$ a unique vector $u \in \operatorname{span}\left[e_{1}, \ldots, e_{k}\right]$ with $\left\langle u, e_{k}\right\rangle=1$ such that $T u=\lambda_{k}(T) u$. We denote this vector by $u_{k}(T)$, so that $T u_{k}(T)=$ $\lambda_{k}(T) u_{k}(T)$. We also denote by $\widetilde{u}_{k}(T)$ the normalized eigenvector $\widetilde{u}_{k}(T)=u_{k}(T) /\left\|u_{k}(T)\right\|$ of $T$. Here is a useful fact concerning the functions $\lambda_{k}$ and $u_{k}$ :

Fact 4.2. - For each $k \geq 1$, the functions $T \mapsto \lambda_{k}(T)$ and $T \mapsto u_{k}(T)$ are continuous from $(\mathfrak{T}(\mathcal{H}), \mathrm{SOT})$ into $\mathbb{T}$ and $\mathcal{H}$ respectively.

Proof. - The continuity of the function $T \mapsto \lambda_{k}(T)$ is obvious from the definition of $\lambda_{k}(T)$. As to the function $T \mapsto u_{k}(T)$, the fact that $\left\langle u_{k}(T), e_{k}\right\rangle=1$ implies that

$$
\left\langle u_{k}(T), e_{i}\right\rangle=\frac{1}{\lambda_{k}(T)-\lambda_{i}(T)} \sum_{j=i+1}^{k}\left\langle T e_{j}, e_{i}\right\rangle\left\langle u_{k}(T), e_{j}\right\rangle
$$

for every $1 \leq i \leq k-1$. A straightforward induction then shows that the scalar functions $T \mapsto\left\langle u_{k}(T), e_{i}\right\rangle, 1 \leq i \leq k-1$, are SOT-continuous on $\mathfrak{T}(\mathcal{H})$, from which Fact 4.2 follows.

Fact 4.3. - Let $u \in \mathcal{H}_{00}$, and choose $r \geq 1$ such that $u \in \operatorname{span}\left[e_{1}, \ldots, e_{r}\right]$. For any $T \in \mathfrak{T}(\mathcal{H}), u$ can be written as $u=\sum_{k=1}^{r} a_{k}(T) u_{k}(T)$, where the functions $T \mapsto a_{k}(T)$, $1 \leq k \leq r$, are continuous from $(\mathfrak{T}(\mathcal{H})$, SOT $)$ into $\mathbb{C}$.

Proof. - This follows directly from Cramer's formulas and the continuity of the maps $T \mapsto u_{k}(T), k \geq 1$.

4.2. Perfect spanning is typical. - The following result shows that within any class $\mathfrak{T}_{M}(\mathcal{H}), M>1$, the perfect spanning property (aka ergodicity in the Gaussian sense) is a typical property with respect to the topology SOT.

Proposition 4.4. - For every $M>1$, the set $\operatorname{PSPAN}_{M}(H)=\mathrm{G}_{-\mathrm{ERG}_{M}(\mathcal{H}) \text { is comea- }}$ ger in $\left(\mathfrak{T}_{M}(\mathcal{H}), \mathrm{SOT}\right)$. Consequently, $\operatorname{ERG}_{M}(H), \mathrm{FHC}_{M}(H)$ and $\mathrm{UFHC}_{M}(H)$ are all comeager in $\left(\mathfrak{T}_{M}(\mathcal{H}), \mathrm{SOT}\right)$.

Proof. - We first recall the following key fact, which goes back to [27, Th. 4.1] (see also [29, Prop. 6.1]).

Fact 4.5. - An operator $T \in \mathfrak{T}(\mathcal{H})$ has a perfectly spanning set of unimodular eigenvectors as soon as the following property is satisfied: for every $\varepsilon>0$ and every $k \geq 1$, there exists $l \geq 1$ with $l \neq k$ and $\alpha \in \mathbb{C}$ such that $\left\|u_{k}(T)-\alpha u_{l}(T)\right\|<\varepsilon$. 
From Fact 4.5, we deduce that the set

$$
\mathfrak{G}=\bigcap_{i \geq 1} \bigcap_{k \geq 1} \bigcup_{l \neq k} \bigcup_{\alpha \in \mathbb{C}}\left\{T \in \mathfrak{T}_{M}(\mathcal{H}) ;\left\|u_{k}(T)-\alpha u_{l}(T)\right\|<2^{-i}\right\}
$$

is contained in $\operatorname{PSPAN}_{M}(H)$. Moreover, it follows from Fact 4.2 that $\mathfrak{G}$ is also a $G_{\delta}$ subset of $\left(\mathfrak{T}_{M}(\mathcal{H})\right.$, SOT). So we just have to prove that $\mathfrak{G}$ is dense in $\mathfrak{T}_{M}(\mathcal{H})$; and for this, it is enough to show that each open set

$$
\mathfrak{O}_{i, k}=\bigcup_{l \neq k} \bigcup_{\alpha \in \mathbb{C}}\left\{T \in \mathfrak{T}_{M}(\mathcal{H}) ;\left\|u_{k}(T)-\alpha u_{l}(T)\right\|<2^{-i}\right\}, \quad i, k \geq 1
$$

is dense in $\left(\mathfrak{T}_{M}(\mathcal{H})\right.$, SOT $)$.

Let us fix $i, k \geq 1$ and $A \in \mathfrak{T}_{M}(\mathcal{H})$. We have to show that for any $r \geq 1$, there exists an operator $T \in \mathfrak{O}_{i, k}$ such that $T e_{j}$ is arbitrarily close to $A e_{j}$ for every $j=1 \ldots, r$. Without loss of generality, we assume that $r \geq k$. Without loss of generality, we can suppose also that $\|A\|<M$. Indeed, if $\|A\|=M$, consider the function $f: t \mapsto\|D+t(A-D)\|$ defined on the compact set $[0,1]$, where $D$ is the diagonal operator given by the diagonal coefficients of $A$. The function $f$ is convex on $[0,1]$, so that its maximum is attained at either 0 or 1 . Since $f(0)=1<M=f(1), f(t)<M$ for every $t \in[0,1)$. If $t \in[0,1)$ is sufficiently close to 1 , the operator $A^{\prime}:=D+t(A-D)$ is upper-triangular with respect to $\left(e_{k}\right)_{k \geq 1}$, has the same diagonal coefficients as $A$, satisfies $\left\|A^{\prime}\right\|<M$, and is as close as we wish to $A$ for the SOT topology. We thus suppose to begin with that $\|A\|<M$.

We set, for every $r \geq 1, \mathcal{H}_{r}:=\operatorname{span}\left[e_{1}, \ldots, e_{r}\right]$, and denote by $P_{r}$ the orthogonal projection of $\mathcal{H}$ onto $\mathcal{H}_{r}$.

For every $1 \leq j \leq r$, we denote by $\lambda_{j}$ the $j$-th diagonal coefficient of $A$ : $\lambda_{j}=\left\langle A e_{j}, e_{j}\right\rangle$. We recall that $\left|\lambda_{j}\right|=1$. Let us denote by $\boldsymbol{\Lambda}_{r}$ the set of all sequences $\boldsymbol{\lambda}=\left(\lambda_{j}\right)_{j>r}$ with $\left|\lambda_{j}\right|=1$ such that all the elements $\lambda_{j}, j>r$, are pairwise distinct and distinct from $1, \lambda_{1}, \ldots, \lambda_{r}$, and $\lambda_{j} \rightarrow 1$ as $j \rightarrow \infty$. For any element $\boldsymbol{\lambda}$ of $\boldsymbol{\Lambda}_{r}$ and any unilateral weight sequence $\boldsymbol{\omega}=\left(\omega_{j}\right)_{j \geq 1}$, we consider the operator $A_{\boldsymbol{\lambda}, \boldsymbol{\omega}}$ on $\mathcal{H}$ defined by

$$
A_{\lambda, \omega} e_{j}= \begin{cases}A e_{j} & \text { for every } 1 \leq j \leq r \\ \omega_{j-r} e_{j-r}+\lambda_{j} e_{j} & \text { for every } j>r .\end{cases}
$$

Note that since $\|A\|<M,\left\|A_{\boldsymbol{\lambda}, \boldsymbol{\omega}}\right\| \leq M$ for every $\boldsymbol{\lambda} \in \boldsymbol{\Lambda}_{r}$ provided $\|\boldsymbol{\omega}\|_{\infty}:=\sup _{j \geq 1}\left|\omega_{j}\right|$ is small enough. Hence in this case $A_{\boldsymbol{\lambda}, \boldsymbol{\omega}}$ belongs to $\mathfrak{T}_{M}(\mathcal{H})$. Moreover, we have $A_{\boldsymbol{\lambda}, \boldsymbol{\omega}} e_{j}=A e_{j}$ for every $j=1, \ldots, r$. Let us now fix a weight sequence $\boldsymbol{\omega}$ such that $\omega_{j} \rightarrow 0$ as $j \rightarrow \infty$ and $\|\boldsymbol{\omega}\|_{\infty}$ is small enough. We are going to show that $A_{\boldsymbol{\lambda}, \boldsymbol{\omega}}$ belongs to $\mathfrak{O}_{i, k}$ provided the sequence $\boldsymbol{\lambda}$ is well-chosen. This will prove the density of $\mathfrak{O}_{i, k}$ in $\left(\mathfrak{T}_{M}(\mathcal{H}), \mathrm{SOT}\right)$.

We first note the following fact concerning the eigenvalues of the operator $A_{\boldsymbol{\lambda}, \boldsymbol{\omega}}$.

Fact 4.6. - For any $\boldsymbol{\lambda} \in \boldsymbol{\Lambda}_{r}$, the only eigenvalues of $A_{\boldsymbol{\lambda}, \boldsymbol{\omega}}$ are $\lambda_{1}, \ldots, \lambda_{r}$, the terms of the sequence $\boldsymbol{\lambda}$, and possibly the point 1 . Moreover, for any $i \geq 1$, the eigenspace associated to $\lambda_{i}$ is spanned by the vector $u_{i}\left(A_{\boldsymbol{\lambda}, \boldsymbol{\omega}}\right)$.

Proof of Fact 4.6. - If $\lambda$ is any complex number, solving formally the equation $A_{\lambda, \omega} x=$ $\lambda x$ with $x=\sum_{j \geq 1} x_{j} e_{j}$, yields that

$$
(A-\lambda) P_{r} x=\sum_{j=1}^{r} \omega_{j} x_{j+r} e_{j} \quad \text { and } \quad x_{j+r}=\frac{\lambda-\lambda_{j}}{\omega_{j}} x_{j} \quad \text { for every } j>r .
$$


Since $\omega_{j} \rightarrow 0$ and $\lambda_{j} \rightarrow 1$ as $j \rightarrow \infty$, we infer from these equations that there is no solution $x \in \mathcal{H} \backslash\{0\}$ to these equations if $\lambda$ does not belong to the set $\left\{\lambda_{j} ; j \geq 1\right\} \cup\{1\}$. This proves the first part of Fact 4.6 ,

For the same reasons combined with the fact that the $\lambda_{j}$ are pairwise distinct and distinct from 1 , we also observe that if a vector $x \in \mathcal{H}$ satisfies $A_{\boldsymbol{\lambda}, \boldsymbol{\omega}} x=\lambda_{i} x$ for some $i \geq 1$, then $x_{j}=0$ for all $j>i$. Hence $\operatorname{ker}\left(A_{\boldsymbol{\lambda}, \boldsymbol{\omega}}-\lambda_{i}\right)$ is contained in $\mathcal{H}_{i}$ for every $i \geq 1$, which proves the second part of Fact 4.6 since $A_{\boldsymbol{\lambda}, \boldsymbol{\omega}}$ belongs to $\mathfrak{T}(\mathcal{H})$ and the eigenvalues $\lambda_{j}$ of $A_{\boldsymbol{\lambda}, \boldsymbol{\omega}}$ are all distinct.

The key point is now the following observation. Recall that $\lambda_{1}, \ldots, \lambda_{r}$ are fixed, that $1 \leq k \leq r$, and that we are especially interested in $\lambda_{k}$. Recall also that $\widetilde{u}_{k+r}\left(A_{\boldsymbol{\lambda}, \boldsymbol{\omega}}\right)=$ $u_{k+r}\left(A_{\boldsymbol{\lambda}, \boldsymbol{\omega}}\right) /\left\|u_{k+r}\left(A_{\boldsymbol{\lambda}, \boldsymbol{\omega}}\right)\right\|$.

Fact 4.7. - There exists a positive constant $\gamma$ such that for any $\boldsymbol{\lambda}=\left(\lambda_{j}\right)_{j>r} \in \boldsymbol{\Lambda}_{r}$,

$$
\left|\lambda_{k+r}-\lambda_{k}\right| \geq \gamma \operatorname{dist}\left(\widetilde{u}_{k+r}\left(A_{\lambda, \omega}\right), \operatorname{span}\left[u_{k}\left(A_{\lambda, \omega}\right)\right]\right) .
$$

Proof. - For any $\boldsymbol{\lambda}=\left(\lambda_{j}\right)_{j>r} \in \boldsymbol{\Lambda}_{r}$, the restriction of the operator $A_{\boldsymbol{\lambda}, \boldsymbol{\omega}}$ to $\mathcal{H}_{r}$ is equal to $P_{r} A P_{r}$, which does not depend on $\boldsymbol{\lambda}$. Also, $\operatorname{ker}\left(A_{\boldsymbol{\lambda}, \boldsymbol{\omega}}-\lambda_{k}\right)=\operatorname{span}\left[u_{k}\left(A_{\boldsymbol{\lambda}, \boldsymbol{\omega}}\right)\right] \subseteq \mathcal{H}_{r}$, and the $r$ eigenvalues $\lambda_{1}, \ldots, \lambda_{r}$ of $P_{r} A P_{r}$ are distinct. These observations imply that there exist two positive constants $\delta$ and $C$ such that the following two properties hold true:

$$
\forall \boldsymbol{\lambda} \in \boldsymbol{\Lambda}_{r} \forall x \in \mathcal{H}_{r}:\left\|\left(A_{\boldsymbol{\lambda}, \boldsymbol{\omega}}-\lambda_{k}\right) x\right\| \geq \delta \operatorname{dist}\left(x, \operatorname{span}\left[u_{k}\left(A_{\boldsymbol{\lambda}, \boldsymbol{\omega}}\right)\right]\right)
$$

and

$$
\forall \lambda \in \mathbb{C} \backslash\left\{\lambda_{1}, \ldots, \lambda_{r}\right\} \forall x \in \mathcal{H}_{r}:\left\|\left(A_{\lambda, \omega}-\lambda\right)^{-1} x\right\| \geq \frac{C}{\operatorname{dist}\left(\lambda,\left\{\lambda_{1}, \ldots, \lambda_{r}\right\}\right)}\|x\| .
$$

Now, a simple computation yields that

$$
\widetilde{u}_{k+r}\left(A_{\lambda, \omega}\right)=\frac{v_{k}+e_{k+r}}{\left(\left\|v_{k}\right\|^{2}+1\right)^{1 / 2}}
$$

where $v_{k} \in \mathcal{H}_{r}$ satisfies the equation $\left(A_{\boldsymbol{\lambda}, \boldsymbol{\omega}}-\lambda_{k+r}\right) v_{k}+\omega_{k} e_{k}=0$. Since $\lambda_{k+r}$ does not belong to the set $\left\{\lambda_{1}, \ldots, \lambda_{r}\right\}$, we may write $v_{k}=-\omega_{k}\left(A_{\lambda, \omega}-\lambda_{k+r}\right)^{-1} e_{k}$, and (9) implies that

$$
\left\|v_{k}\right\| \geq C \frac{\left|\omega_{k}\right|}{\operatorname{dist}\left(\lambda_{k+r},\left\{\lambda_{1}, \ldots, \lambda_{r}\right\}\right)} \geq C \frac{\left|\omega_{k}\right|}{\left|\lambda_{k+r}-\lambda_{k}\right|} .
$$

On the other hand, $\left(A_{\lambda, \omega}-\lambda_{k}\right) v_{k}=\left(\lambda_{k+r}-\lambda_{k}\right) v_{k}-\omega_{k} e_{k}$, so that by (8)

$$
\left|\lambda_{k+r}-\lambda_{k}\right|\left\|v_{k}\right\|+\left|\omega_{k}\right| \geq \delta \operatorname{dist}\left(v_{k}, \operatorname{span}\left[u_{k}\left(A_{\boldsymbol{\lambda}, \boldsymbol{\omega}}\right)\right]\right) .
$$

Hence

$$
\operatorname{dist}\left(v_{k}+e_{k+r}, \operatorname{span}\left[u_{k}\left(A_{\lambda, \omega}\right)\right]\right) \leq \frac{1}{\delta}\left(\left|\lambda_{k+r}-\lambda_{k}\right|\left\|v_{k}\right\|+\left|\omega_{k}\right|\right)+1
$$


and

$$
\begin{aligned}
\operatorname{dist}\left(\widetilde{u}_{k+r}\left(A_{\lambda, \omega}\right),\right. & \left.\operatorname{span}\left[u_{k}\left(A_{\lambda, \omega}\right)\right]\right) \\
& \leq \frac{1}{\delta}\left(\left|\lambda_{k+r}-\lambda_{k}\right| \frac{\left\|v_{k}\right\|}{\left(\left\|v_{k}\right\|^{2}+1\right)^{1 / 2}}+\frac{\left|\omega_{k}\right|}{\left(\left\|v_{k}\right\|^{2}+1\right)^{1 / 2}}\right)+\frac{1}{\left(\left\|v_{k}\right\|^{2}+1\right)^{1 / 2}} \\
& \leq \frac{1}{\delta}\left(\left|\lambda_{k+r}-\lambda_{k}\right|+\frac{\left|\omega_{k}\right|}{\left\|v_{k}\right\|}\right)+\frac{1}{\left\|v_{k}\right\|} \\
& \leq\left|\lambda_{k+r}-\lambda_{k}\right|\left(\frac{1}{\delta}+\frac{1}{C}+\frac{1}{C\left|\omega_{k}\right|}\right) \quad \text { by (10). }
\end{aligned}
$$

Setting $1 / \gamma:=1 / \delta+1 / C+1 /\left(C \min _{1 \leq j \leq r}\left|\omega_{j}\right|\right)$ yields the desired inequality.

We now come back to our main proof: recall that our aim is to show that $A_{\boldsymbol{\lambda}, \boldsymbol{\omega}}$ belongs to $\mathfrak{O}_{i, k}$ for some suitable choice of $\lambda \in \boldsymbol{\Lambda}_{r}$. By Fact 4.7, we have

$$
\operatorname{dist}\left(\widetilde{u}_{k+r}\left(A_{\boldsymbol{\lambda}, \boldsymbol{\omega}}\right), \operatorname{span}\left[u_{k}\left(A_{\boldsymbol{\lambda}, \boldsymbol{\omega}}\right)\right]\right) \leq \frac{\left|\lambda_{k+r}-\lambda_{k}\right|}{\gamma} \text { for every } \boldsymbol{\lambda} \in \boldsymbol{\Lambda}_{r} .
$$

We now choose $\boldsymbol{\lambda} \in \boldsymbol{\Lambda}_{r}$ such that $\lambda_{k+r}$ is so close to $\lambda_{k}$ that the quantity on the right hand side of the inequality (11) is less that $2^{-i} \eta_{k}$, where $\eta_{k}=\min \left(1,\left(2\left\|u_{k}\left(A_{\boldsymbol{\lambda}, \boldsymbol{\omega}}\right)\right\|\right)^{-1}\right)$. Note that $\eta_{k}$ does not depend on $\boldsymbol{\lambda}$ since the restriction of $A_{\boldsymbol{\lambda}, \boldsymbol{\omega}}$ to $\mathcal{H}_{r}$ does not. With this choice of $\boldsymbol{\lambda}$, there exists a scalar $\beta \in \mathbb{C}$ such that $\left\|\widetilde{u}_{k+r}\left(A_{\boldsymbol{\lambda}, \boldsymbol{\omega}}\right)-\beta u_{k}\left(A_{\boldsymbol{\lambda}, \boldsymbol{\omega}}\right)\right\|<2^{-i} \eta_{k}$. Since $\left\|\widetilde{u}_{k+r}\left(A_{\boldsymbol{\lambda}, \boldsymbol{\omega}}\right)\right\|=1$ and $2^{-i} \eta_{k} \leq 1 / 2$, we have $\left\|\beta u_{k}\left(A_{\boldsymbol{\lambda}, \boldsymbol{\omega}}\right)\right\| \geq 1 / 2$, so that $|\beta| \geq \eta_{k}$. It follows that $\left\|\alpha u_{k+r}\left(A_{\boldsymbol{\lambda}, \boldsymbol{\omega}}\right)-u_{k}\left(A_{\boldsymbol{\lambda}, \boldsymbol{\omega}}\right)\right\|<2^{-i}$, where $\alpha:=1 /\left(\beta\left\|u_{k+r}\left(A_{\boldsymbol{\lambda}, \boldsymbol{\omega}}\right)\right\|\right)$, and this shows that $A_{\lambda, \omega}$ belongs to $\mathfrak{O}_{i, k}$.

Thus we have proved that $\mathfrak{O}_{i, k}$ is indeed dense in $\left(\mathfrak{T}_{M}(\mathcal{H})\right.$, SOT) for every $i, k \geq 1$, which concludes the proof of Proposition 4.4.

Remark 4.8. - It is natural to wonder whether the class of chaotic operators is also comeager in $\mathfrak{T}_{M}(\mathcal{H})$. This does not look quite clear from the above proof.

4.3. Ergodicity vs ergodicity in the Gaussian sense. - Let $\left(e_{k}\right)_{k \geq 1}$ be as usual a fixed orthonormal basis of $\mathcal{H}$. In this subsection, we focus on special operators of the associated class $\mathfrak{T}(\mathcal{H})$ of upper-triangular operators with respect to $\left(e_{k}\right)_{k \geq 1}$, which are the sum of a diagonal operator with respect to the basis $\left(e_{k}\right)_{k>1}$ and a backward weighted shift operator with respect to this same basis. More precisely, we introduce the following notation: we denote by $\boldsymbol{\Lambda}$ the set of sequences $\boldsymbol{\lambda}=\left(\lambda_{k}\right)_{k \geq 1}$ of pairwise distinct complex numbers of modulus 1 such that $\left(\lambda_{k}\right)_{k \geq 1}$ tends to 1 as $k$ tends to infinity, and for every $M>0$, by $\boldsymbol{\Omega}_{M}$ the set of all weight sequences $\boldsymbol{\omega}=\left(\omega_{k}\right)_{k \geq 1}$ such that $0<\omega_{k} \leq M$ for every $k \geq 1$. We also set $\Omega:=\bigcup_{M>0} \Omega_{M}$.

We endow the set $\boldsymbol{\Lambda}$ with the topology induced by $\ell^{\infty}(\mathbb{N})$. Since $\boldsymbol{\Lambda}$ is contained in the separable closed subspace $c(\mathbb{N})$ of $\ell^{\infty}(\mathbb{N})$ consisting of all convergent sequences, and since $\Lambda$ is easily seen to be a $G_{\delta}$ subset of $\ell^{\infty}(\mathbb{N})$, it follows that $\Lambda$ is a Polish space. As for the spaces $\boldsymbol{\Omega}_{M}, M>0$, we endow them with the product topology. Each $\boldsymbol{\Omega}_{M}$ being a $G_{\delta}$ subset of $\mathbb{R}^{\mathbb{N}}$, it is thus a Polish space as well.

To each pair $(\boldsymbol{\lambda}, \boldsymbol{\omega}) \in \boldsymbol{\Lambda} \times \boldsymbol{\Omega}$, we associate the operator $T_{\boldsymbol{\lambda}, \boldsymbol{\omega}}$ defined by $T_{\boldsymbol{\lambda}, \boldsymbol{\omega}}=D_{\boldsymbol{\lambda}}+B_{\boldsymbol{\omega}}$, where $D_{\boldsymbol{\lambda}}$ is the diagonal operator with diagonal coefficients $\lambda_{k}, k \geq 1$, associated to the basis $\left(e_{k}\right)_{k \geq 1}$, and $B_{\boldsymbol{\omega}}$ is the backward shift operator with respect to $\left(e_{k}\right)_{k \geq 1}$ with weights $\omega_{k}, k \geq 1$. Each operator $T_{\boldsymbol{\lambda}, \boldsymbol{\omega}}$ belongs to $\mathfrak{T}(\mathcal{H})$. 
In this subsection, our aim is to investigate the properties of the operator $T_{\lambda \omega}$ for a typical choice of parameters $(\boldsymbol{\lambda}, \boldsymbol{\omega}) \in \boldsymbol{\Lambda} \times \boldsymbol{\Omega}$. For every $M>0$, we consider the following two sets of parameters:

$$
\mathcal{E}_{M}:=\left\{(\boldsymbol{\lambda}, \boldsymbol{\omega}) \in \boldsymbol{\Lambda} \times \boldsymbol{\Omega}_{M} ; T_{\boldsymbol{\lambda}, \boldsymbol{\omega}} \text { is ergodic }\right\}
$$

and

$$
\mathcal{D}_{M}:=\left\{(\boldsymbol{\lambda}, \boldsymbol{\omega}) \in \boldsymbol{\Lambda} \times \boldsymbol{\Omega}_{M} ; \sigma_{p}\left(T_{\boldsymbol{\lambda}, \boldsymbol{\omega}}\right) \subseteq\left\{\lambda_{k}, k \geq 1\right\} \cup\{1\}\right\},
$$

where $\sigma_{p}(T)$ denotes the point spectrum (i.e. the set of eigenvalues) of an operator $T \in$ $\mathfrak{B}(\mathcal{H})$. The operators belonging to $\mathcal{D}_{M}$ are those which have the smallest possible set of eigenvalues among the operators $T_{\boldsymbol{\lambda}, \boldsymbol{\omega}},(\boldsymbol{\lambda}, \boldsymbol{\omega}) \in \boldsymbol{\Lambda} \times \boldsymbol{\Omega}_{M}$. In particular, they have countable unimodular point spectrum.

Our main result can now be stated as follows:

Theorem 4.9. - For any $M>2$, the two sets $\mathcal{E}_{M}$ and $\mathcal{D}_{M}$ are comeager in $\boldsymbol{\Lambda} \times \boldsymbol{\Omega}_{M}$.

As an immediate consequence, we obtain

Corollary 4.10. - There exist ergodic operators on $\mathcal{H}$, of the form $T_{\boldsymbol{\lambda}, \boldsymbol{\omega}},(\boldsymbol{\lambda}, \boldsymbol{\omega}) \in \boldsymbol{\Lambda} \times \boldsymbol{\Omega}$, which have countable unimodular point spectrum. In particular, these operators are ergodic but not ergodic in the Gaussian sense.

Remark 4.11. - Corollary 4.10 provides examples of hypercyclic operators on a Hilbert space with a spanning set of unimodular eigenvectors but only countably many unimodular eigenvalues. The question of the existence of such operators was first raised by Flytzanis in [22], and answered recently in [39]. Indeed, the chaotic non-frequently hypercyclic operators constructed there have only countably many eigenvalues, which are all roots of unity, and the associated eigenvectors span the space. Corollary 4.10 strengthens this result by showing that ergodic counterexamples to Flytzanis' conjecture exist, and that such counterexamples are in some sense much less exotic than suggested by the rather technical construction of [39].

Remark 4.12. - Since all the operators we are considering here have infinitely many eigenvalues, Corollary 4.10 leaves open the question of the existence of ergodic operators on $\mathcal{H}$ without any eigenvalue at all. Such operators are known to exist on the Banach space $\mathcal{C}_{0}([0,2 \pi])$ of continuous functions on $[0,2 \pi]$ vanishing at the point 0 . Indeed, the Kalisch operator $T$ defined by

$$
T f(\theta)=e^{i \theta} f(\theta)-\int_{0}^{\theta} i e^{i t} f(t) d t
$$

when considered as acting on $\mathcal{C}_{0}([0,2 \pi])$, is ergodic in the Gaussian sense but does not admit any unimodular eigenvalue; see [5] or [7, Section 5.5.4] for details. On the other hand, an operator on a Hilbert space which is ergodic in the Gaussian sense definitely has a lot of unimodular eigenvalues (recall that $\mathrm{G}-\operatorname{ERG}(\mathcal{H})=\operatorname{PSPAN}(\mathcal{H})$ ); but a general ergodic operator might possibly have no eigenvalue at all.

We now turn to the proof of Theorem 4.9, We first state a simple fact, in which a complete description of the unimodular eigenvectors of the operators $T_{\boldsymbol{\lambda}, \boldsymbol{\omega}}$ is given. 
Fact 4.13. - Fix $(\boldsymbol{\lambda}, \boldsymbol{\omega}) \in \boldsymbol{\Lambda} \times \boldsymbol{\Omega}$, and $\lambda \in \mathbb{C}$. Then $\lambda$ is an eigenvalue of $T_{\boldsymbol{\lambda}, \boldsymbol{\omega}}$ if and only if the vector

$$
E_{\boldsymbol{\lambda}, \omega}(\lambda):=e_{1}+\sum_{n \geq 2}\left(\prod_{j=1}^{n-1} \frac{\lambda-\lambda_{j}}{\omega_{j}}\right) e_{n}
$$

is a well-defined vector of $\mathcal{H}$. In this case, $\operatorname{ker}\left(T_{\boldsymbol{\lambda}, \boldsymbol{\omega}}-\lambda\right)=\operatorname{span}\left[E_{\boldsymbol{\lambda}, \boldsymbol{\omega}}(\lambda)\right]$.

Proof. - It suffices to solve formally the equation $T_{\lambda, \omega} x=\lambda x$ in $\mathbb{C}^{\mathbb{N}}$.

For every $k \geq 1$,

$$
E_{\lambda, \omega}\left(\lambda_{k}\right)=e_{1}+\sum_{n=2}^{k}\left(\prod_{j=1}^{n-1} \frac{\lambda_{k}-\lambda_{j}}{\omega_{j}}\right) e_{n}
$$

is thus an eigenvector of $T_{\boldsymbol{\lambda}, \boldsymbol{\omega}}$ associated to the eigenvalue $\lambda_{k}$, and hence is proportional to $u_{k}\left(T_{\boldsymbol{\lambda}, \boldsymbol{\omega}}\right)$.

The next lemma provides necessary conditions for the point spectrum of $T_{\lambda, \omega}$ to be either "maximal" or "minimal". For any element $\boldsymbol{\omega}$ of $\boldsymbol{\Omega}$, we denote by $R_{\boldsymbol{\omega}}$ the radius of convergence of the series

$$
\sum_{j \geq 1} \frac{z^{j}}{\omega_{1} \cdots \omega_{j}} .
$$

Lemma 4.14. - Let $(\boldsymbol{\lambda}, \boldsymbol{\omega})$ be an element of $\boldsymbol{\Lambda} \times \boldsymbol{\Omega}$.

(1) If $R_{\boldsymbol{\omega}}>2$, the map $\lambda \mapsto E_{\boldsymbol{\lambda}, \boldsymbol{\omega}}(\lambda)$ is well-defined and analytic on a neighborhood of the unit circle $\mathbb{T}$. Consequently, $\overline{\operatorname{span}}\left[E_{\boldsymbol{\lambda}, \boldsymbol{\omega}}\left(\lambda_{k}\right) ; k>k_{0}\right]=\mathcal{H}$ for every $k_{0} \geq 1$.

(2) If $R_{\boldsymbol{\omega}}=0$, the eigenvalues of $T_{\boldsymbol{\lambda}, \boldsymbol{\omega}}$ are contained in the set $\left\{\lambda_{k} ; k \geq 1\right\} \cup\{1\}$.

Proof. - The first part of assertion (1) is a direct consequence of Fact 4.13; since $R_{\omega}>2$, the series defining $E_{\boldsymbol{\lambda}, \omega}(\lambda)$ is convergent in $\mathcal{H}$ for every $\lambda$ belonging to the open disk $D\left(0, R_{\omega}-1\right)$, and the map $\lambda \mapsto E_{\lambda, \omega}(\lambda)$ is analytic there. In particular, this map is analytic in a neighborhood of $\mathbb{T}$. Since the sequence $\left(\lambda_{k}\right)_{k>k_{0}}$ has an accumulation point in $D\left(0, R_{\boldsymbol{\omega}}-1\right)$ for every $k_{0} \geq 1$, it follows from the analyticity of $E_{\boldsymbol{\lambda}, \boldsymbol{\omega}}$ that the closed linear span of the vectors $E_{\boldsymbol{\lambda}, \boldsymbol{\omega}}\left(\lambda_{k}\right), k>k_{0}$, coincides with the closed linear span of all the eigenvectors $E_{\boldsymbol{\lambda}, \boldsymbol{\omega}}(\lambda), \lambda \in \mathbb{T}$. Since $E_{\boldsymbol{\lambda}, \boldsymbol{\omega}}\left(\lambda_{k}\right)$ is proportional to $u_{k}\left(T_{\boldsymbol{\lambda}, \boldsymbol{\omega}}\right)$ and since the vectors $u_{k}\left(T_{\boldsymbol{\lambda}, \omega}\right), k \geq 1$, span a dense subspace of $\mathcal{H}$, this proves the second part of assertion (1).

As to assertion (2), suppose that $R_{\boldsymbol{\omega}}=0$. Let us show that the series defining $E_{\boldsymbol{\lambda}, \boldsymbol{\omega}}(\lambda)$ does not converge when $\lambda \in \mathbb{C}$ does not belong to $\left\{\lambda_{k} ; k \geq 1\right\} \cup\{1\}$. So let fix such a complex number $\lambda$. Since $\lambda_{k}$ tends to 1 as $k$ tends to infinity, there exists a number $\delta>0$ such that $\left|\lambda-\lambda_{k}\right| \geq \delta$ for every $k \geq 1$. Hence

$$
\left|\prod_{j=1}^{n-1} \frac{\lambda-\lambda_{j}}{\omega_{j}}\right|^{2} \geq \frac{\delta^{2(n-1)}}{\left(\omega_{1} \ldots \omega_{n-1}\right)^{2}} \quad \text { for every } n \geq 2 .
$$

Since $R_{\omega}=0$, it follows that

$$
\sum_{n \geq 2}\left|\prod_{j=1}^{n-1} \frac{\lambda-\lambda_{j}}{\omega_{j}}\right|^{2}=\infty
$$

and hence $E_{\boldsymbol{\lambda}, \boldsymbol{\omega}}(\lambda)$ is not defined as a vector of $\mathcal{H}$. 
After these preliminary facts, we now state a proposition which will be the key step to prove that ergodic elements are typical in $\boldsymbol{\Lambda} \times \boldsymbol{\Omega}_{M}$ for any $M>2$. We postpone the proof of Proposition 4.15 to the end of this section, and explain first how Theorem 4.9 may be deduced from it. In what follows, we fix $M>2$. For any $\boldsymbol{\omega} \in \boldsymbol{\Omega}_{M}$, any open set $U \neq \emptyset$ in $\mathcal{H}$ and any $\alpha>0$, we set

$$
\begin{aligned}
\mathcal{O}_{U, \alpha}^{\omega}:=\{\boldsymbol{\lambda} \in \boldsymbol{\Lambda} ; & \exists r \geq 1 \exists\left(a_{1}, \ldots, a_{r}\right) \in \mathbb{C}^{r}: \\
& \left.\sum_{k=1}^{r} a_{k} u_{k}\left(T_{\boldsymbol{\lambda}, \boldsymbol{\omega}}\right) \in U \text { and } \sum_{k=1}^{r}\left|a_{k}\right|^{2}\left\|u_{k}\left(T_{\boldsymbol{\lambda}, \boldsymbol{\omega}}\right)\right\|^{2}<\alpha\right\} .
\end{aligned}
$$

Note that $\mathcal{O}_{U, \alpha}^{\omega}$ is an open subset of $\boldsymbol{\Lambda}$ : this follows from the continuity of the map $(\boldsymbol{\lambda}, \boldsymbol{\omega}) \mapsto T_{\boldsymbol{\lambda}, \boldsymbol{\omega}}$ from $\boldsymbol{\Lambda} \times \boldsymbol{\Omega}_{M}$ into $\left(\mathfrak{T}_{M+1}(\mathcal{H})\right.$, SOT $)$, combined with Fact 4.2 ,

Proposition 4.15. - Let $U$ be a non-empty open set in $\mathcal{H}$, and let $\alpha>0$. If $\boldsymbol{\omega} \in \boldsymbol{\Omega}_{M}$ is such that $R_{\boldsymbol{\omega}}>2$, then the open set $\mathcal{O}_{U, \alpha}^{\omega}$ is dense in $\boldsymbol{\Lambda}$.

Proposition 4.15 will allow us to apply the following ergodicity criterion proved in [29, Cor. 5.5].

Lemma 4.16. - Let $X$ be a separable Banach space, and let $T \in \mathfrak{B}(X)$. Assume that for any open set $\Omega \neq \emptyset$ of $X$ with $T^{-1}(\Omega) \subseteq \Omega$, any neighborhood $W$ of 0 , and any $\varepsilon>0$, there exists a T-invariant probability measure $\mu$ on $X$ with compact support such that $\mu(\Omega)=1$ and $\mu(W)>1-\varepsilon$. Then $T$ is ergodic.

The link with Proposition 4.15 is perhaps not quite obvious at first sight. It is provided by the following fact.

Fact 4.17. - Let $T \in \mathfrak{T}(\mathcal{H})$, let $U$ be a non-empty open set in $\mathcal{H}$, let $\varepsilon>0$, and let $W$ be a neighborhood of 0 in $\mathcal{H}$. Let also $r \geq 1$, and assume that the eigenvalues $\lambda_{1}(T), \ldots, \lambda_{r}(T)$ of $T$ are rationally independent. Finally, let $a_{1}, \ldots, a_{r}$ be $r$ complex numbers and assume that the vector $\sum_{k=1}^{r} a_{k} u_{k}(T)$ belongs to $U$. If the quantity $\sum_{k=1}^{r}\left|a_{k}\right|^{2}\left\|u_{k}(T)\right\|^{2}$ is small enough, there exists a compactly supported $T$-invariant measure $\mu$ on $\mathcal{H}$ such that

- $\mu\left(\Omega_{U}\right)=1$, where $\Omega_{U}=\bigcup_{n \geq 0} T^{-n}(U)$, and

- $\mu(W)>1-\varepsilon$.

Proof. - We only sketch the proof, since the argument is already essentially given in 29. Let $\left(\chi_{k}\right)_{k=1}^{r}$ be a sequence of independent random Steinhaus variables defined on some standard probability space $(\Omega, \mathcal{F}, \mathbb{P})$, and let $\mu$ be the measure on $\mathcal{H}$ given by the distribution of the $\mathcal{H}$-valued random variable $\sum_{k=1}^{r} \chi_{k} a_{k} u_{k}(T)$. This measure $\mu$, which may be called the Steinhaus measure associated with the vector $u=\sum_{k=1}^{r} a_{k} u_{k}(T)$, is $T$-invariant and has compact support. As observed in [29. Fact 5.16], the independence of the unimodular numbers $\lambda_{1}(T), \ldots, \lambda_{r}(T)$ and the condition $\sum_{k=1}^{r} a_{k} u_{k}(T) \in U$ imply that $\mu\left(\Omega_{U}\right)=1$. Also, we have by orthogonality of the Steinhaus variables $\chi_{k}, 1 \leq k \leq r$, that

$$
\int_{H}\|x\|^{2} d \mu(x)=\mathbb{E}\left(\sum_{k=1}^{r} \chi_{k} a_{k} u_{k}(T)\right)=\sum_{k=1}^{r}\left|a_{k}\right|^{2}\left\|u_{k}(T)\right\|^{2} .
$$

It then follows from Markov's inequality that $\mu(W)>1-\varepsilon$ provided that the quantity $\sum_{k=1}^{r}\left|a_{k}\right|^{2}\left\|u_{k}(T)\right\|^{2}$ is sufficiently small. 
Proof of Theorem 4.9. - The proofs of the two parts of Theorem 4.9 are completely independent of each other. Recall that the sets $\mathcal{E}_{M}$ and $\mathcal{D}_{M}$ are defined at the beginning of Section 4.3, and that we assume that $M>2$.

Part 1. The set $\mathcal{E}_{M}$ is comeager in $\boldsymbol{\Lambda} \times \boldsymbol{\Omega}_{M}$.

Let $\left(U_{p}\right)_{p \geq 1}$ be a countable basis of non-empty open subsets for $\mathcal{H}$. For each $\boldsymbol{\omega} \in \boldsymbol{\Omega}_{M}$, define the $G_{\delta}$ subset of $\boldsymbol{\Lambda}$

$$
\boldsymbol{G}_{\boldsymbol{\omega}}:=\bigcap_{p \geq 1} \bigcap_{q \geq 1} \mathcal{O}_{U_{p}, 2^{-q}}^{\omega}
$$

and

$$
G:=\left\{(\lambda, \omega) \in \Lambda \times \Omega ; \lambda \in G_{\omega}\right\} .
$$

Claim 4.18. - The set $\boldsymbol{G}$ is a dense $G_{\delta}$ subset of $\boldsymbol{\Lambda} \times \boldsymbol{\Omega}_{M}$.

Proof of Claim 4.18, - The fact that $G$ is $G_{\delta}$ in $\Lambda \times \Omega_{M}$ is immediate. As to its density, we first observe that since $M>2$, Proposition 4.15 implies that $\boldsymbol{G}_{\boldsymbol{\omega}}$ is dense in $\boldsymbol{\Lambda}$ as soon as $\omega_{j}=M$ for $j$ sufficiently large. It follows that the set $\left\{\boldsymbol{\omega} \in \boldsymbol{\Omega}_{M} ; \boldsymbol{G}_{\boldsymbol{\omega}}\right.$ is dense in $\left.\boldsymbol{\Lambda}\right\}$ is dense in $\boldsymbol{\Omega}_{M}$. Since this set is also clearly $G_{\delta}$ in $\boldsymbol{\Omega}_{M}$, it is thus comeager in $\boldsymbol{\Omega}_{M}$. The Kuratowski-Ulam theorem then implies that the $G_{\delta}$ set $G$ is comeager and hence dense in $\Lambda \times \Omega_{M}$.

It is now easy to show that the set $\mathcal{E}_{M}$ is comeager in $\boldsymbol{\Lambda} \times \boldsymbol{\Omega}_{M}$. Indeed, it follows from Lemma 4.16 and Fact 4.17 that $T_{\boldsymbol{\lambda}, \boldsymbol{\omega}}$ is ergodic as soon as $\boldsymbol{\lambda}$ belongs to $\boldsymbol{G}_{\boldsymbol{\omega}}$ and the terms $\lambda_{k}, k \geq 1$, of the sequence $\boldsymbol{\lambda}$ are rationally independent. Hence the set

$$
\boldsymbol{G}_{\text {ind }}=\left\{(\boldsymbol{\lambda}, \boldsymbol{\omega}) \in \boldsymbol{G} \text {; the unimodular numbers } \lambda_{k}, k \geq 1 \text {, are rationally independent }\right\}
$$

is contained in $\mathcal{E}_{M}$. Since the conditions that the numbers $\lambda_{k}$ should be rationally independent is easily seen to define a dense $G_{\delta}$ subset of $\boldsymbol{\Lambda}$, Claim 4.18 implies that $\boldsymbol{G}_{\text {ind }}$ is a dense $G_{\delta}$ subset of $\boldsymbol{\Lambda} \times \boldsymbol{\Omega}_{M}$. Hence $\mathcal{E}_{M}$ is comeager in $\boldsymbol{\Lambda} \times \boldsymbol{\Omega}_{M}$.

Part 2. The set $\mathcal{D}_{M}$ is comeager in $\boldsymbol{\Lambda} \times \boldsymbol{\Omega}_{M}$.

The argument here is much simpler, and relies solely on part (2) of Lemma 4.14. Observe that the set of all $\boldsymbol{\omega} \in \boldsymbol{\Omega}_{M}$ such that $R_{\boldsymbol{\omega}}=\liminf _{j \rightarrow \infty}\left(\omega_{1} \ldots \omega_{j}\right)^{1 / j}=0$ is $G_{\delta}$ in $\boldsymbol{\Omega}_{M}$, and that this set is dense in $\boldsymbol{\Omega}_{M}$ because it contains all sequences $\boldsymbol{\omega} \in \boldsymbol{\Omega}_{M}$ such that $\omega_{j}$ tends to 0 as $j$ tends to infinity. Hence the set $\left\{(\boldsymbol{\lambda}, \boldsymbol{\omega}) \in \boldsymbol{\Lambda} \times \boldsymbol{\Omega}_{M} ; R_{\boldsymbol{\omega}}=0\right\}$ is $G_{\delta}$ and dense in $\Lambda \times \Omega_{M}$, and the claim follows.

We now turn to the proof of Proposition 4.15. Again, it relies on some arguments used in [29], more precisely in the proof of [29, Th. 5.12].

Proof of Proposition 4.15. - Let us fix $\boldsymbol{\theta} \in \boldsymbol{\Lambda}$ and $\varepsilon>0$. We are looking for an element $\boldsymbol{\lambda}$ of $\mathcal{O}_{U, \alpha}^{\omega}$ such that $\|\boldsymbol{\lambda}-\boldsymbol{\theta}\|_{\infty}<\varepsilon$.

Writing $\boldsymbol{\theta}$ as $\boldsymbol{\theta}=\left(\theta_{k}\right)_{k>1}$, we fix $k_{0} \geq 1$ such that $\left|\theta_{k}-1\right|<\varepsilon / 3$ for every $k>k_{0}$. By assertion (1) of Lemma 4.14, the linear span of the vectors $E_{\boldsymbol{\theta}, \boldsymbol{\omega}}\left(\theta_{s}\right), s>k_{0}$, is dense in $\mathcal{H}$, so there exists an index $Q \geq 1$ and complex numbers $b_{k_{0}+1}, \ldots, b_{k_{0}+q}$ such that the vector

$$
z:=\sum_{s=k_{0}+1}^{k_{0}+Q} b_{s} E_{\boldsymbol{\theta}, \omega}\left(\theta_{s}\right) \text { belongs to } U \text {. }
$$


We now proceed as in 27 or 29, and replace each coefficient $b_{s}$ by a certain sum of scalars of the form $\sum_{j=0}^{N-1} c_{s+j Q}$ with $\sum_{j=0}^{N-1}\left|c_{s+j Q}\right|^{2}$ sufficiently small. More precisely, we define

$$
c_{s+j Q}=\frac{1}{N} b_{s} \quad \text { and } \quad v_{s+j Q}=E_{\boldsymbol{\theta}, \boldsymbol{\omega}}\left(\theta_{s}\right)
$$

for every $k_{0}+1 \leq s \leq k_{0}+Q$ and every $0 \leq j \leq N-1$, where $N \geq 1$ is an integer so large that

$$
\sum_{k=k_{0}+1}^{k_{0}+N Q}\left|c_{k}\right|^{2}\left\|v_{k}\right\|^{2}=\frac{1}{N} \sum_{s=k_{0}+1}^{k_{0}+Q}\left|b_{s}\right|^{2}\left\|E_{\boldsymbol{\theta}, \omega}\left(\theta_{s}\right)\right\|^{2}<\alpha .
$$

Observe that $\sum_{k=k_{0}+1}^{k_{0}+N Q} c_{k} v_{k}=z$ by construction, so that we have

$$
\sum_{k=k_{0}+1}^{k_{0}+N Q} c_{k} v_{k} \in U
$$

The next step in the proof is to define $\boldsymbol{\lambda} \in \boldsymbol{\Lambda}$ with $\|\boldsymbol{\lambda}-\boldsymbol{\theta}\|_{\infty}<\varepsilon$, in such a way that each vector $v_{k}, k_{0}+1 \leq k \leq k_{0}+N Q$, can be approximated by the eigenvector $E_{\boldsymbol{\lambda}, \boldsymbol{\omega}}\left(\lambda_{k}\right)$ of $T_{\lambda, \omega}$ associated to the eigenvalue $\lambda_{k}$, and the $\lambda_{k}, k_{0}+1 \leq k \leq k_{0}+N Q$, are all distinct. The sequence $\boldsymbol{\lambda}$ is defined as follows: we keep $\lambda_{k}=\theta_{k}$ for every $1 \leq k \leq k_{0}+Q$ and every $k>k_{0}+N Q$, so that in particular $\lambda_{k} \rightarrow 1$ as $k \rightarrow \infty$. For $k_{0}+1 \leq s \leq k_{0}+Q$ and $1 \leq j \leq N-1$, we choose $\lambda_{s+j Q}$ extremely close to $\theta_{s}$, in such a way that these new coefficients $\lambda_{k}$ are all distinct and distinct from all the $\theta_{k}$. Since we already know that $\lambda_{k} \rightarrow 1$ as $k \rightarrow \infty$, the sequence $\boldsymbol{\lambda}$ defined in this way belongs to $\boldsymbol{\Lambda}$. We can certainly ensure that $\left|\lambda_{s+j+1}-\theta_{s}\right|<\varepsilon$ for all $k_{0}+1 \leq s \leq k_{0}+Q$ and $1 \leq j \leq N-1$, and thus that

$$
\left|\lambda_{s+j Q}-\theta_{s+j Q}\right|<\varepsilon / 3+\left|\theta_{s+j Q}-1\right|+\left|\theta_{s}-1\right|<\varepsilon
$$

since $s>k_{0}$. All the remaining coefficients of $\boldsymbol{\lambda}$ and $\boldsymbol{\theta}$ coincide, and hence $\|\boldsymbol{\lambda}-\boldsymbol{\theta}\|_{\infty}<\varepsilon$.

Let us now show that the quantities

$$
\left\|E_{\boldsymbol{\lambda}, \omega}\left(\lambda_{s+j Q}\right)-v_{s+j Q}\right\|=\left\|E_{\boldsymbol{\lambda}, \omega}\left(\lambda_{s+j Q}\right)-E_{\boldsymbol{\theta}, \boldsymbol{\omega}}\left(\theta_{s}\right)\right\|,
$$

where $k_{0}+1 \leq s \leq k_{0}+Q$ and $0 \leq j \leq N-1$, can be made as small as we wish, provided each coefficient $\lambda_{s+j Q}$ is close enough to $\theta_{s}$. We consider separately two cases.

- Assume that $k_{0}+1 \leq s \leq k_{0}+Q$ and $j=0$. In this case $\lambda_{s}=\theta_{s}$ by definition, so that $E_{\boldsymbol{\lambda}, \boldsymbol{\omega}}\left(\lambda_{s}\right)=E_{\boldsymbol{\theta}, \boldsymbol{\omega}}\left(\theta_{s}\right)$.

- Assume now that $k_{0}+1 \leq s \leq k_{0}+Q$ and $1 \leq j \leq N-1$. In this case, we use the explicit expression of the eigenvectors provided by Fact 4.13 and write

$$
\begin{aligned}
E_{\boldsymbol{\lambda}, \boldsymbol{\omega}}\left(\lambda_{s+j Q}\right)-E_{\boldsymbol{\theta}, \boldsymbol{\omega}}\left(\theta_{s}\right)=\sum_{n=2}^{s}[ & \left.\prod_{i=1}^{n-1}\left(\frac{\lambda_{s+j Q}-\lambda_{i}}{\omega_{i}}\right)-\prod_{i=1}^{n-1}\left(\frac{\theta_{s}-\theta_{i}}{\omega_{i}}\right)\right] e_{n} \\
& +\sum_{n=s+1}^{s+j Q} \prod_{i=1}^{n-1}\left(\frac{\lambda_{s+j Q}-\lambda_{i}}{\omega_{i}}\right) e_{n} .
\end{aligned}
$$

Fix an arbitrarily small number $\delta>0$. The integers $s$ and $j$ being fixed, and the numbers $\lambda_{i}$ and $\theta_{i}$ being by definition equal for every $1 \leq i \leq k_{0}+Q$, the norm of the first sum in the above expression can be made less that $\delta / 2$, provided that the difference $\left|\lambda_{s+j Q}-\theta_{s}\right|$ is sufficiently small. As for the second term, observe that $s$ belongs to the set $\{1, \ldots, n-1\}$ for every $s+1 \leq n \leq s+j Q$, so that the term $\lambda_{s+j Q}-\lambda_{s}=\lambda_{s+j Q}-\theta_{s}$ always appears in 
the product $\prod_{i=1}^{n-1}\left(\lambda_{s+j Q}-\lambda_{i}\right) / \omega_{i}$. Thus if $\left|\lambda_{s+j Q}-\theta_{s}\right|$ is sufficiently small, the norm of the second term is less than $\delta / 2$ too. Hence one can ensure that $\left\|E_{\boldsymbol{\lambda}, \boldsymbol{\omega}}\left(\lambda_{s+j Q}\right)-E_{\boldsymbol{\theta}, \boldsymbol{\omega}}\left(\theta_{s}\right)\right\|<\delta$ for all $k_{0}+1 \leq s \leq k_{0}+Q$ and $1 \leq j \leq N-1$.

So we have proved that for any $\delta>0$, one can construct $\boldsymbol{\lambda} \in \boldsymbol{\Lambda}$ such that $\|\boldsymbol{\lambda}-\boldsymbol{\theta}\|_{\infty}<\varepsilon$ and $\left\|E_{\boldsymbol{\lambda}, \boldsymbol{\omega}}\left(\lambda_{k}\right)-v_{k}\right\|<\delta$ for every $k_{0}+1 \leq k \leq k_{0}+N Q$. If $\delta$ is now chosen small enough, the two conditions

$$
\sum_{k=k_{0}+1}^{k_{0}+N Q} c_{k} E_{\boldsymbol{\lambda}, \boldsymbol{\omega}}\left(\lambda_{k}\right) \in U \text { and } \sum_{k=k_{0}+1}^{k_{0}+N Q}\left|c_{k}\right|^{2}\left\|E_{\boldsymbol{\lambda}, \boldsymbol{\omega}}\left(\lambda_{k}\right)\right\|^{2}<\alpha
$$

simultaneously hold true, by (13) and (12) respectively. Remembering that each vector $u_{k}\left(T_{\boldsymbol{\lambda}, \boldsymbol{\omega}}\right)$ is proportional to $E_{\boldsymbol{\lambda}, \boldsymbol{\omega}}\left(\lambda_{k}\right)$, we eventually obtain that there exist complex coefficients $a_{k}, k_{0}+1 \leq k \leq k_{0}+N Q$, such that

$$
\sum_{k=k_{0}+1}^{k_{0}+N Q} a_{k} u_{k}\left(T_{\boldsymbol{\lambda}, \boldsymbol{\omega}}\right) \in U \text { and } \sum_{k=k_{0}+1}^{k_{0}+N Q}\left|a_{k}\right|^{2}\left\|u_{k}\left(T_{\boldsymbol{\lambda}, \boldsymbol{\omega}}\right)\right\|^{2}<\alpha .
$$

Hence $\boldsymbol{\lambda}$ belongs to $\mathcal{O}_{U, \alpha}^{\omega}$, and this concludes the proof of Proposition 4.15,

Remark 4.19. - In order to show that the ergodic operators are comeager in $\Lambda \times \Omega_{M}$, we have used in a crucial way the fact that the terms $\lambda_{k}, k \geq 1$, of the sequences $\boldsymbol{\lambda} \in \boldsymbol{\Lambda}$ involved in the proof are rationally independent. This is not so surprising in view, for instance, of [29, Fact 5.16]. Yet the role of independence in these issues remains rather mysterious. We develop this a little bit in the next subsection.

4.4. Additional remarks. - In this subsection, we present a short discussion of some questions motivated by the results obtained above, as well as some further results concerning dynamical properties of the "diagonal plus shift" operators $T_{\boldsymbol{\lambda}, \boldsymbol{\omega}}$.

4.4.1. Some natural questions. - Two such questions concerning the existence of operators on a Hilbert space with particular ergodic-theoretic-like properties remain unanswered at this stage of our work. The first one is

Question 4.20. - Do there exist $\mathcal{U}$-frequently hypercyclic operators on $\mathcal{H}$ which are not frequently hypercyclic?

It was proved by Bayart and Rusza in $[9$ that such operators do exist on the space $c_{0}$, but the question was left open for Hilbert (or even reflexive) spaces. In the light of the discussion carried out in Section 3 of this paper, it seems natural to conjecture that the two classes $\operatorname{UFHC}(\mathcal{H})$ and $\operatorname{FHC}(\mathcal{H})$ should have different descriptive complexity, and hence should be distinct; but we have been unable to solve the question using this approach. The second question runs as follows:

Question 4.21. - Do there exist frequently hypercyclic operators on $\mathcal{H}$ which are not ergodic?

This question comes from [29, where it is proved that frequently hypercyclic nonergodic operators do exist on the space $c_{0}$. The proof of this result again relies on a construction of $\mathbf{9}$ : it is proved in $\mathbf{2 9}$ that the frequently hypercyclic bilateral weighted shifts $T$ on $c_{0}(\mathbb{Z})$ defined in 9 satisfy $c(T)<1$, and thus cannot be ergodic. For some 
reasons, we found it rather tempting to try to attack this question by Baire category methods; but again, we did not succeed in this way.

We do solve Questions 4.20 and 4.21 in Section 6 below, but using widely different methods. The operators we will use are generalizations of those introduced by the third named author in 39 in order to solve the question of the existence of a chaotic nonfrequently hypercyclic operator. The two main results we will obtain are that indeed, there exist $\mathcal{U}$-frequently hypercyclic operators on $\mathcal{H}$ which are not frequently hypercyclic, as well as frequently hypercyclic operators which are not ergodic. All our examples turn out to be chaotic; and we will complement these results by showing that there also exist on $\mathcal{H}$ operators which are chaotic and topologically mixing but not $\mathcal{U}$-frequently hypercyclic.

In another direction, the role of rational independence in all that concerns the links between unimodular eigenvalues and ergodicity properties of operators needs to be clarified. To be a little more specific, let us consider the class $\mathfrak{T}_{\text {ind }}(\mathcal{H})$ of operators in $\mathfrak{T}(\mathcal{H})$ whose diagonal coefficients are rationally independent. Rather surprisingly, it seems that very little is known concerning such operators. For example, to our knowledge the following question is open:

Question 4.22. - Let $T$ be a hypercyclic operator belonging to $\mathfrak{T}_{\text {ind }}(\mathcal{H})$. Is $T$ necessarily ergodic? frequently hypercyclic? $\mathcal{U}$-frequently hypercyclic?

The only currently known examples of hypercyclic operators with spanning unimodular eigenvectors which are not frequently hypercyclic or not ergodic are those constructed in [39] and the ones that will be considered in Section [6. As already mentioned, all these operators are chaotic. Moreover, for many of these operators, the only unimodular eigenvalues are roots of unity and for some of those, each eigenvalue has multiplicity one. So these operators belong to $\mathfrak{T}(\mathcal{H})$ for some suitably chosen orthonormal basis $\left(e_{k}\right)_{k \geq 1}$, but not to $\mathfrak{T}_{\text {ind }}(\mathcal{H})$ for any basis $\left(e_{k}\right)_{k \geq 1}$. A positive answer to Question 4.22 would show that this is not accidental, i.e. that there must be a strong amount of dependence between the eigenvalues of any of the operators constructed in [39] or in Section 6] of the present paper.

As a matter of fact, Question 4.22 seems to be open even for the operators $T_{\lambda, \omega}=$ $D_{\boldsymbol{\lambda}}+B_{\boldsymbol{\omega}}$ considered in Subsection 4.3. Even more prosaically, it seems quite desirable (and perhaps not too difficult) to determine when exactly an operator of the form $T_{\boldsymbol{\lambda}, \boldsymbol{\omega}}$ is hypercyclic.

4.4.2. More on the operators $T_{\lambda, \omega}$. - We finish this section by collecting some simple facts that we do know concerning operators of the form $T_{\boldsymbol{\lambda}, \boldsymbol{\omega}}$. Let us introduce the following notations.

- If $\boldsymbol{\omega}$ is a unilateral weight sequence, we set $a_{1}(\boldsymbol{\omega}):=1$ and

$$
a_{j}(\boldsymbol{\omega}):=\frac{1}{\omega_{1} \cdots \omega_{j-1}} \quad \text { for every } j \geq 2 .
$$

Also, using the same notation as in Section 4.3 above, we denote by $R_{\boldsymbol{\omega}}$ the radius of convergence of the series $\sum a_{j}(\boldsymbol{\omega}) z^{j}$ :

$$
R_{\boldsymbol{\omega}}=\liminf _{j \rightarrow \infty}\left|\omega_{1} \cdots \omega_{j-1}\right|^{1 / j} .
$$


- To each $\boldsymbol{\lambda} \in \mathbb{T}^{\mathbb{N}}$, we associate polynomials $P_{\boldsymbol{\lambda}, j}, j \geq 1$, defined as follows: $P_{\boldsymbol{\lambda}, 1}(\lambda) \equiv 1$ and

$$
P_{\boldsymbol{\lambda}, j}(\lambda)=\prod_{k=1}^{j-1}\left(\lambda-\lambda_{k}\right) \quad \text { for every } j \geq 2 .
$$

Proposition 4.23. - Let $\boldsymbol{\omega}$ be a unilateral weight sequence, and let $\boldsymbol{\lambda} \in \mathbb{T}^{\mathbb{N}}$. Then the following facts hold true.

(0) A complex number $\lambda$ is an eigenvalue of $T_{\boldsymbol{\lambda}, \omega}$ if and only if

$$
\sum_{j=1}^{\infty}\left|a_{j}(\boldsymbol{\omega})\right|^{2}\left|P_{j}(\lambda)\right|^{2}<\infty .
$$

In this case, $\operatorname{ker}(T-\lambda)=\operatorname{span}\left[E_{\boldsymbol{\lambda}, \boldsymbol{\omega}}(\lambda)\right]$ where

$$
E_{\boldsymbol{\lambda}, \boldsymbol{\omega}}(\lambda)=\sum_{j=1}^{\infty} a_{j}(\boldsymbol{\omega}) P_{\boldsymbol{\lambda}, j}(\lambda) e_{j}
$$

(1) If $R_{\boldsymbol{\omega}}=0$, then $\sigma_{p}\left(T_{\lambda, \omega}\right) \subseteq \overline{\left\{\lambda_{k} ; k \geq 1\right\}}$.

(2) If $\sigma\left(B_{\boldsymbol{\omega}}\right)=\{0\}$ (e.g. if $\omega_{j} \rightarrow 0$ as $j \rightarrow \infty$ ), then $\sigma\left(T_{\boldsymbol{\lambda}, \boldsymbol{\omega}}\right) \subseteq \overline{\left\{\lambda_{k} ; k \geq 1\right\}}$. More generally, $\sigma\left(T_{\lambda, \omega}\right)$ is contained in the set $\left\{\lambda \in \mathbb{C}\right.$; $\left.\operatorname{dist}\left(\lambda,\left\{\lambda_{k}\right\}\right) \leq r\left(B_{\boldsymbol{\omega}}\right)\right\}$, where $r\left(B_{\boldsymbol{\omega}}\right)$ denotes the spectral radius of $B_{\boldsymbol{\omega}}$.

Assume now that $\boldsymbol{\lambda}$ belongs to $\boldsymbol{\Lambda}$, i.e. that the elements $\lambda_{k}, k \geq 1$, are pairwise distinct and that $\lambda_{k} \rightarrow 1$ as $k \rightarrow \infty$.

(3) If $R_{\boldsymbol{\omega}}>0$, then $\sigma_{p}\left(T_{\boldsymbol{\lambda}, \boldsymbol{\omega}}\right)$ contains $\left\{\lambda_{k} ; k \geq 1\right\} \cup D\left(1, R_{\boldsymbol{\omega}}\right)$ and is contained in $\left\{\lambda_{k} ; k \geq 1\right\} \cup \bar{D}\left(1, R_{\omega}\right)$.

(4) If $R_{\boldsymbol{\omega}}>\sup _{k \geq 1}\left|\lambda_{k}-1\right|$, then $T_{\boldsymbol{\lambda}, \boldsymbol{\omega}}$ is ergodic in the Gaussian sense. Moreover, the map $\lambda \mapsto E_{\lambda, \omega}(\lambda)$ is analytic on the open disk $D\left(0, R_{\boldsymbol{\omega}}-1\right)$.

(5) If $R_{\boldsymbol{\omega}}<\sup _{k \geq 1}\left|\lambda_{k}-1\right|$, then $T_{\boldsymbol{\lambda}, \boldsymbol{\omega}}$ is not hypercyclic.

(6) If $R_{\boldsymbol{\omega}}=\sup _{k \geq 1}\left|\lambda_{k}-1\right|$, let $k_{0}$ be the largest integer $k \geq 1$ such that $\left|\lambda_{k}-1\right| \geq R_{\boldsymbol{\omega}}$. If

$$
\sum_{j \geq k_{0}+1}\left|\frac{\omega_{1} \cdots \omega_{j-1}}{\left(\lambda_{k_{0}}-\lambda_{k_{0}+1}\right) \cdots\left(\lambda_{k_{0}}-\lambda_{j}\right)}\right|^{2}<\infty,
$$

then $T_{\boldsymbol{\lambda}, \boldsymbol{\omega}}$ is not ergodic in the Gaussian sense.

Proof. - (0) This is a simple computation which has already been carried out in the proof of Fact 4.13 .

(1) If $\lambda \in \mathbb{C}$ does not belong to the closure of $\left\{\lambda_{k} ; k \geq 1\right\}$, then $\inf _{k \geq 1}\left|\lambda-\lambda_{k}\right|=\delta>0$, and thus $\left|P_{j}(\lambda)\right| \geq \delta^{j-1}$ for all $j \geq 1$. If $R_{\boldsymbol{\omega}}=0$, assertion (0) implies that $\lambda$ is not an eigenvalue of $T_{\lambda, \omega}$.

(2) Let $\lambda \in \mathbb{C}$. We have $T_{\boldsymbol{\lambda}, \boldsymbol{\omega}}-\lambda I=D_{\boldsymbol{\xi}}+B_{\boldsymbol{\omega}}$, where $\boldsymbol{\xi}=\left(\xi_{k}\right)_{k \geq 1}$ is defined by $\xi_{k}=\lambda_{k}-\lambda$ for every $k \geq 1$. If $\delta:=\operatorname{dist}\left(\lambda,\left\{\lambda_{k}\right\}\right)>0$, then $D_{\boldsymbol{\xi}}$ is invertible and $T_{\boldsymbol{\lambda}, \boldsymbol{\omega}}-\lambda I$ can be written as

$$
T_{\lambda, \omega}-\lambda I=D_{\boldsymbol{\xi}}\left(I+D_{\boldsymbol{\xi}}^{-1} B_{\boldsymbol{\omega}}\right)=D_{\boldsymbol{\xi}}\left(I+B_{\boldsymbol{\xi}^{-1} \boldsymbol{\omega}}\right)
$$

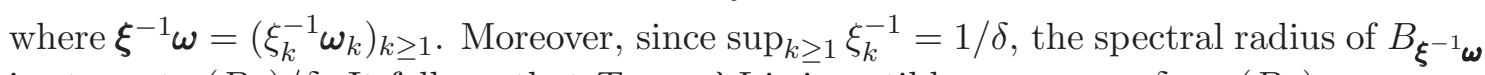
is at most $r\left(B_{\boldsymbol{\omega}}\right) / \delta$. It follows that $T_{\boldsymbol{\lambda}, \boldsymbol{\omega}}-\lambda I$ is invertible as soon as $\delta>r\left(B_{\boldsymbol{\omega}}\right)$. 
(3) We already know by assertion (0) that $\sigma_{p}\left(T_{\boldsymbol{\lambda}, \boldsymbol{\omega}}\right)$ contains $\left\{\lambda_{k} ; k \geq 1\right\}$. Now, let $\lambda$ belong to the open disk $D\left(1, R_{\boldsymbol{\omega}}\right)$, and choose $r$ such that $|\lambda-1|<r<R_{\boldsymbol{\omega}}$. Since $\lambda_{k} \rightarrow 1$, there exists $k_{0} \geq 1$ such that $\left|\lambda-\lambda_{k}\right|<r$ for all $k \geq k_{0}$. It follows that there exists a constant $C>0$ such that $\left|P_{j}(\lambda)\right| \leq C r^{j}$ for every $j \geq 1$, so that $E_{\boldsymbol{\lambda}, \boldsymbol{\omega}}(\lambda)$ is well-defined and analytic on $D\left(1, R_{\boldsymbol{\omega}}\right)$.

Conversely, assume that $\lambda$ does not belong to the set $\left\{\lambda_{k} ; k \geq 1\right\} \cup \bar{D}\left(1, R_{\boldsymbol{\omega}}\right)$. Since $\lambda_{k} \rightarrow 1$, one can find $r>R_{\boldsymbol{\omega}}$ such that $\left|\lambda-\lambda_{k}\right| \geq r$ for $k$ sufficiently large; and since $\lambda \neq \lambda_{k}$ for all $k \geq 1$, it follows that there exists a constant $c>0$ such that $\left|P_{j}(\lambda)\right| \geq c r^{j}$ for every $j \geq 1$. Hence $E_{\lambda, \omega}(\lambda)$ is not well-defined.

(4) By assertion (3), the map $\lambda \mapsto E_{\lambda, \omega}(\lambda)$ is analytic on the disk $D\left(1, R_{\omega}\right)$; so it is enough to show that the vectors $E_{\lambda, \boldsymbol{\omega}}(\lambda), \lambda \in D\left(1, R_{\boldsymbol{\omega}}\right)$, span a dense subspace of $\mathcal{H}$. Let $y=\sum_{j \geq 1} y_{j} e_{j}$ be a vector of $\mathcal{H}$ which is orthogonal to all the vectors $E_{\boldsymbol{\lambda}, \boldsymbol{\omega}}(\lambda)$, $\lambda \in D\left(1, R_{\omega}\right)$. We thus have

$$
\sum_{j=1}^{\infty} \bar{y}_{j} a_{j}(\boldsymbol{\omega}) P_{\boldsymbol{\lambda}, j}(\lambda)=0 \quad \text { for every } \lambda \in D\left(1, R_{\boldsymbol{\omega}}\right) .
$$

The series above is convergent on the disk $D\left(1, R_{\boldsymbol{\omega}}\right)$, which contains all the points $\lambda_{k}$ by assumption. So

$$
\sum_{j=1}^{\infty} \bar{y}_{j} a_{j}(\boldsymbol{\omega}) P_{\boldsymbol{\lambda}, j}\left(\lambda_{k}\right)=0 \quad \text { for every } k \geq 1 .
$$

Since $P_{\boldsymbol{\lambda}, j}\left(\lambda_{k}\right)=0$ whenever $j>k$ and $P_{\boldsymbol{\lambda}, k}\left(\lambda_{k}\right) \neq 0$, it follows that $\bar{y}_{j} a_{j}(\boldsymbol{\omega})=0$ for every $j \geq 1$, so that $y=0$.

(5) Let $k_{0} \geq 1$ be such that $\left|\lambda_{k_{0}}-1\right|>R_{\boldsymbol{\omega}}$. Then $\lambda_{k_{0}}$ is an isolated eigenvalue of $T_{\boldsymbol{\lambda}, \boldsymbol{\omega}}$ by assertion (3), which is easily seen not to belong to the essential spectrum of $T_{\boldsymbol{\lambda}, \boldsymbol{\omega}}$. It then follows from [34] that $T_{\boldsymbol{\lambda}, \boldsymbol{\omega}}$ cannot be hypercyclic.

(6) By assertion (1), we can assume that $R_{\boldsymbol{\omega}}>0$, since otherwise the point spectrum $\sigma_{p}\left(T_{\boldsymbol{\lambda}, \boldsymbol{\omega}}\right)$ of $T_{\boldsymbol{\lambda}, \boldsymbol{\omega}}$ is countable, and $T_{\boldsymbol{\lambda}, \boldsymbol{\omega}}$ is certainly not ergodic in the Gaussian sense in this case. It suffices to show that the unimodular eigenvectors of $T_{\lambda, \boldsymbol{\omega}}$ are not perfectly spanning. Since $\left(\sigma_{p}(T) \cap \mathbb{T}\right) \backslash D\left(1, R_{\boldsymbol{\omega}}\right)$ is a finite set by assertion (3), it is in turn enough to show that the vectors $E_{\boldsymbol{\lambda}, \boldsymbol{\omega}}(\lambda), \lambda \in D\left(1, R_{\boldsymbol{\omega}}\right)$, do not span a dense subspace of $\mathcal{H}$. So we have to find coefficients $c_{j}, j \geq 1$, with the property that

$$
\sum_{j=1}^{\infty} c_{j} P_{\boldsymbol{\lambda}, j}(\lambda)=0 \quad \text { for every } \lambda \in D\left(1, R_{\boldsymbol{\omega}}\right),
$$

with the additional requirement that

$$
\sum_{j=1}^{\infty}\left|\frac{c_{j}}{a_{j}(\boldsymbol{\omega})}\right|^{2}<\infty, \quad \text { i.e. that } \quad \sum_{j \geq 2}\left|c_{j} \omega_{1} \cdots \omega_{j-1}\right|^{2}<\infty .
$$

We define the coefficients $c_{j}$ by setting $c_{j}=0$ for every $1 \leq j<k_{0}, c_{k_{0}}=1$ and

$$
c_{j}=\prod_{i=k_{0}+1}^{j}\left(\lambda_{k_{0}}-\lambda_{i}\right)^{-1} \quad \text { for every } j>k_{0} .
$$


Then condition (14) is satisfied by assumption. Moreover, the coefficients $c_{j}$ have been defined in such a way that

$$
\sum_{j=1}^{N} c_{j} P_{\boldsymbol{\lambda}, j}(\lambda)=P_{\boldsymbol{\lambda}, k_{0}}(\lambda) \cdot \prod_{k=k_{0}+1}^{N}\left(\frac{\lambda-\lambda_{k}}{\lambda_{k_{0}}-\lambda_{k}}\right) \quad \text { for all } N \geq k_{0}+1 .
$$

Since $\left|\lambda_{k_{0}}-1\right| \geq R_{\boldsymbol{\omega}}$ and $\lambda_{k} \rightarrow 1$ as $k \rightarrow \infty$, it follows that $\sum_{j=1}^{\infty} c_{j} P_{\boldsymbol{\lambda}, j}(\lambda)=0$ for every $\lambda$ such that $|\lambda-1|<R_{\omega}$. This completes the proof of assertion (6).

\section{Periodic points at the service of hypercyclicity}

In this section, we depart from our standing assumption and work in the general context of Banach spaces over $\mathbb{K}=\mathbb{R}$ or $\mathbb{C}$, not restricting ourselves to the Hilbertian setting. Actually, most of our results hold in the framework of arbitrary Polish topological vector spaces, as should be clear from the proofs.

Let thus $X$ be a real or complex separable Banach space. Our aim is to obtain general sufficient conditions for an operator $T \in \mathfrak{B}(X)$ to be frequently hypercyclic or $\mathcal{U}$-frequently hypercyclic. These criteria are rather different from the classical ones, since they involve the periodic points of the operator $T$. They will greatly contribute to simplify some proofs in the next section, and they might hopefully be useful elsewhere also. For any bounded operator $T$ on $X$, we denote by $\operatorname{Per}(T)$ the set of its periodic points, and by $\operatorname{per}_{T}(x)$, or simply $\operatorname{per}(x)$, the period of a periodic point $x$ of $T$.

5.1. Precompact orbits and topological mixing. - We start by giving a very simple criterion for an operator $T \in \mathfrak{B}(X)$ to be topologically mixing, which involves the points of $X$ with precompact orbit under the action of $T$. We denote by $\operatorname{Prec}(T)$ the set of points with precompact orbit.

Proposition 5.1. - Let $T \in \mathfrak{B}(X)$. Assume that $\operatorname{Prec}(T)$ is dense in $X$ and that for every neighborhood $W$ of 0 in $X$ and every non-empty open subset $V$ of $X$, the set $\mathcal{N}_{T}(W, V)$ is cofinite. Then $T$ is topologically mixing.

Proof. - It suffices to show that for any vectors $y \in \operatorname{Prec}(T), x \in X$, and any $\varepsilon>0$, the set $\mathcal{N}_{T}(B(y, \varepsilon), B(x, \varepsilon))$ is cofinite. Since $y$ is a vector with precompact orbit, there exists a finite subset $\left\{y_{1}, \ldots, y_{d}\right\}$ of $X$ such that any point of $\operatorname{Orb}(T, y)$ lies within distance less than $\varepsilon / 2$ of the set $\left\{y_{1}, \ldots, y_{d}\right\}$. In other words, there exists for any $n \geq 1$ an index $i_{n} \in\{1, \ldots, d\}$ such that $\left\|T^{n} y-y_{i_{n}}\right\|<\varepsilon / 2$.

By assumption, each set $\mathcal{N}_{T}\left(B(0, \varepsilon), B\left(x-y_{i}, \varepsilon / 2\right)\right)$ is cofinite. Hence, one can find an integer $N$ such that for every $n \geq N$ and every $1 \leq i \leq d$, there exists $z_{n, i} \in X$ such that

$$
\left\|z_{n, i}\right\|<\varepsilon \quad \text { and } \quad\left\|T^{n} z_{n, i}-\left(x-y_{i}\right)\right\|<\varepsilon / 2 .
$$

For every $n \geq N$, we then have

$$
\left\|T^{n}\left(y+z_{n, i_{n}}\right)-x\right\| \leq\left\|T^{n} y-y_{i_{n}}\right\|+\left\|T^{n} z_{n, i_{n}}-\left(x-y_{i_{n}}\right)\right\|<\varepsilon .
$$

Hence $y+z_{n, i_{n}}$ belongs to $B(y, \varepsilon)$ and $T^{n}\left(y+z_{n, i_{n}}\right)$ belongs to $B(x, \varepsilon)$, which shows that every integer $n \geq N$ lies in the set $\mathcal{N}_{T}((B(y, \varepsilon), B(x, \varepsilon))$.

Since periodic points have a finite orbit, Proposition 5.1 immediately implies the following result. 
Corollary 5.2. - Let $T \in \mathfrak{B}(X)$. If $\operatorname{Per}(T)$ is dense in $X$ and if for every neighborhood $W$ of 0 in $X$ and every non-empty open subset $V$ of $X$, the set $\mathcal{N}_{T}(W, V)$ is cofinite, then $T$ is chaotic and topologically mixing.

We also obtain in a similar fashion.

Corollary 5.3. - Let $T \in \mathfrak{B}(X)$. If there exists a dense set of points $x \in X$ such that $T^{i} x \rightarrow 0$ as $i \rightarrow \infty$ and if for every neighborhood $W$ of 0 in $X$ and every non-empty open subset $V$ of $X$, the set $\mathcal{N}_{T}(W, V)$ is cofinite, then $T$ is topologically mixing.

Finally, the linearity assumption on the system $(X, T)$ implies the following strengthened version of Proposition 5.1.

Corollary 5.4. - Let $T \in \mathfrak{B}(X)$. Assume that $\operatorname{Prec}(T)$ is dense in $X$ and that there exists a subset $X_{0}$ of $X$ with dense linear span such that the following property holds true: for every $x_{0} \in X_{0}$ and every $\varepsilon>0$, there exists $N \geq 1$ such that for every $n \geq N$, one can find $z \in X$ such that $\|z\|<\varepsilon$ and $\left\|T^{n} z-x_{0}\right\|<\varepsilon$. Then $T$ is topologically mixing.

Proof. - Let $V \subseteq X$ be a non-empty open set. Since $X_{0}$ spans a dense subspace of $X$, there exist $\varepsilon>0$ and $x=\sum_{k=1}^{K} \alpha_{k} x_{k} \in V$ with $x_{k} \in X_{0}$ and $\alpha_{k} \neq 0$ for every $1 \leq k \leq K$ such that $B(x, \varepsilon)$ is contained in $V$. Moreover, by assumption, there exists $N \geq 1$ such that for every $1 \leq k \leq K$, every $n \geq N$, one can find $z_{n, k} \in X$ such that

$$
\left\|z_{n, k}\right\|<\frac{\varepsilon}{K\left|\alpha_{k}\right|} \quad \text { and } \quad\left\|T^{n} z_{n, k}-x_{k}\right\|<\frac{\varepsilon}{K\left|\alpha_{k}\right|} .
$$

The vector $z:=\sum_{k=1}^{K} \alpha_{k} z_{n, k}$ then satisfies $\|z\|<\varepsilon$ and $\left\|T^{n} z-x\right\|<\varepsilon$, and the desired result follows from Proposition 5.1

Remark 5.5. - Unlike in Corollary 5.8 below, it is not possible, in Proposition 5.1, to replace the assumption that all the sets $\mathcal{N}_{T}(W, V)$ are cofinite ( $W$ and $V$ non-empty open subsets of $X$ with $0 \in W)$ by the assumption that all the sets $\mathcal{N}_{T}(U, W)$ are cofinite $(W$ and $U$ non-empty open subsets of $X$ with $0 \in W$ ), even if one assumes additionally that $T$ is hypercyclic. Indeed, every unilateral weighted backward shift has a dense set of points with finite orbit and is such that $\mathcal{N}_{T}(U, W)$ is cofinite for every neighborhood $W$ of 0 and every non-empty open set $U$; but there exist hypercyclic weighted shifts which are not topologically mixing.

5.2. Uniform recurrence and topological weak mixing. - In this subsection, we give a simple criterion for an operator $T \in \mathfrak{B}(X)$ to be weakly topologically mixing. It involves the uniformly recurrent points of $T$. Recall that a point $x \in X$ is said to be recurrent for $T$ if, for any neighborhood $O$ of $x$, the set $\mathcal{N}_{T}(x, O)=\left\{i \in \mathbb{N} ; T^{i} x \in O\right\}$ is non-empty (or, equivalently, infinite). A point $x \in X$ is said to be uniformly recurrent for $T$ if, for any neighborhood $O$ of $x$, the set $\mathcal{N}_{T}(x, O)$ has bounded gaps. For example, every periodic point is obviously uniformly recurrent. The following more general fact will be useful. Recall first that a compact, $T$-invariant subset $K$ of $X$ is said to be minimal if it has no proper (non-empty) closed $T$-invariant subset. For example, if $x$ is a periodic point of $T$ with period $N$, then $K:=\left\{x, T x, \ldots, T^{N-1} x\right\}$ is minimal. Recall also that we denote by $\mathcal{E}(T)$ the set of all unimodular eigenvectors of $T$. 
Fact 5.6. - Let $T \in \mathfrak{B}(X)$. If $K$ is a compact minimal $T$-invariant subset of $X$, then every point of $K$ is uniformly recurrent for $T$. In particular, every point $z \in \operatorname{span} \mathcal{E}(T)$ is uniformly recurrent for $T$.

Proof. - The first part of the statement is well-known (and has nothing to do with linearity; see e.g. [23. Theorem 1.15]). As for the second part, it is enough to show that if $z$ belongs to $\operatorname{span} \mathcal{E}(T)$, then its closed $T$-orbit $K=\overline{\left\{T^{n} z ; n \geq 0\right\}}$ is a compact minimal $T$-invariant set.

Write $z$ as $z=\sum_{j=1}^{N} u_{j}$, where for every $1 \leq j \leq N, T u_{j}=\gamma_{j} u_{j}$ for some $\gamma_{j} \in \mathbb{T}$. Let $\Gamma$ be the closed subgroup of $\mathbb{T}^{N}$ generated by the $N$-tuple $\gamma:=\left(\gamma_{1}, \ldots, \gamma_{N}\right)$. Then $K=\left\{\sum_{j=1}^{N} \omega_{j} u_{j} ; \omega=\left(\omega_{1}, \ldots, \omega_{N}\right) \in \Gamma\right\}$, so that $K$ is compact (and, of course, $T$ invariant). So we just have to check that $K$ is minimal for the action of $T$. Let $a \in K$ be arbitrary. We have to show that any point $y \in K$ can be approximated as close as we wish by points of the form $T^{n} a, n \geq 0$. Write $a$ and $y$ as $a=\sum_{j=1}^{N} \omega_{j} u_{j}$ and $y=\sum_{j=1}^{N} \eta_{j} u_{j}$ respectively, where $\omega=\left(\omega_{1}, \ldots, \omega_{N}\right)$ and $\eta=\left(\eta_{1}, \ldots, \eta_{N}\right)$ belong to $\Gamma$. For any integer $n \geq 0$, we have $T^{n} a=\sum_{j=1}^{N} \gamma_{j}^{n} \omega_{j} u_{j}$. Since the $N$-tuples $\gamma^{n}=\left(\gamma_{1}^{n}, \ldots, \gamma_{N}^{n}\right), n \geq 0$, are dense in $\Gamma$ (this follows from the fact that since the group $\mathbb{T}^{N}$ is compact, the closed semigroup generated by $\gamma$ is in fact a group), there exists an integer $n \geq 0$ such that $\gamma^{n}$ is as close as we wish to $\omega^{-1} \eta=\left(\omega_{1}^{-1} \eta_{1}, \ldots, \omega_{N}^{-1} \eta_{N}\right)$. Then $T^{n} a$ is as close as we wish to $y$, as required.

We can now state our criterion for topological weak mixing.

Proposition 5.7. - Let $T \in \mathfrak{B}(X)$, and let $Z$ be a $T$-invariant subset of $X$ consisting of uniformly recurrent points. Assume that for every non-empty open subset $V$ of $X$ and every neighborhood $W$ of 0 in $X$, one can find a vector $z \in Z$ and an integer $n \geq 0$ such that $z \in W$ and $T^{n} z \in V$. Then $T$ is topologically weakly mixing and $Z$ is dense in $X$.

Proof. - That $Z$ is dense in $X$ is clear from the assumption since $Z$ is $T$-invariant. In order to prove that $T$ is topologically weakly mixing, we are going to show that $\mathcal{N}_{T}(U, W) \cap$ $\mathcal{N}_{T}(W, V)$ is non-empty for any non-empty open subsets $U, V$ of $X$ and any neighborhood $W$ of 0 in $X$. The so-called three open sets condition (see for example [7, Ch. 4]) will then imply that $T$ is topologically weakly mixing.

Let us first show that for any neighborhood $W$ of 0 and any open set $V \neq \emptyset$, the set $\mathcal{N}_{T}(W, V)$ has bounded gaps. Choose a vector $z \in Z$ and an integer $n \geq 1$ such that $z$ belongs to $W$ and $T^{n} z$ belongs to $V$, and then an open neighborhood $O$ of $z$ such that $O \subseteq W$ and $T^{n}(O) \subseteq V$. Since $z$ is uniformly recurrent, the set $\mathcal{N}_{T}(z, O)$ has bounded gaps. Then $n+\mathcal{N}_{T}(z, O)$ has bounded gaps as well, and the result follows since $n+\mathcal{N}_{T}(z, O) \subseteq \mathcal{N}_{T}(z, V) \subseteq \mathcal{N}_{T}(W, V)$.

We show next that $\mathcal{N}_{T}(U, W)$ is non-empty for any non-empty open subset $U$ of $X$ and any neighborhood $W$ of 0 in $X$. By assumption, one can find a point $x \in W$ and an integer $n \geq 1$ such that $x$ is a (uniformly) recurrent point of $T$ and $u:=T^{n} x$ lies in $U$. Since $x$ is recurrent, one can find an integer $p>n$ such that $T^{p} x$ lies in $W$. Then $T^{p-n} u=T^{p} x$ belongs to $W$, and hence $\mathcal{N}_{T}(U, W)$ is non-empty.

It is now easy to conclude the proof: all the sets $\mathcal{N}_{T}(W, V)$ have bounded gaps, and all the sets $\mathcal{N}_{T}(U, W)$ contain arbitrarily large intervals, because they are all non-empty and the sets $W$ are neighborhoods of the fixed point 0 . Hence any set of the form $\mathcal{N}_{T}(W, V)$ meets any other set of the form $\mathcal{N}_{T}(U, W)$. 
As a first consequence of Proposition [5.7, we show that for operators having a dense set of uniformly recurrent points, topological weak mixing is equivalent to some formally much weaker "transitivity-like" properties.

Corollary 5.8. - Let $T \in \mathfrak{B}(X)$, and assume that uniformly recurrent points of $T$ are dense in $X$. The following assertions are then equivalent:

(a) $T$ is topologically weakly mixing;

(b) for every non-empty open subset $V$ of $X$ and every neighborhood $W$ of 0 in $X$, the set $\mathcal{N}_{T}(W, V)$ is non-empty;

(c) for every non-empty open subset $U$ of $X$ and every neighborhood $W$ of 0 in $X$, the set $\mathcal{N}_{T}(U, W)$ is non-empty.

Proof. - Obviously, (a) implies both (b) and (c). Moreover, since the uniformly recurrent points of $T$ are assumed to be dense in $X$, it follows at once from Proposition 5.7 (taking as $Z$ the set of all uniformly recurrent points for $T$ ) that (b) implies (a). So it remains to show that (c) implies (b).

Suppose that (c) holds true, and let $U$ and $W$ be two non-empty open subsets of $X$ with $0 \in W$. By (c) and since uniformly recurrent points of $T$ are dense in $X$, one can find a uniformly recurrent point $u \in U$ and an integer $n \geq 0$ such that $z:=T^{n} u$ belongs to $W$. Since $u$ is in particular recurrent, one can find $p>n$ such that $T^{p} u$ belongs to $U$. Then $T^{p-n} z=T^{p} u$ lies in $U$, so that $\mathcal{N}_{T}(W, U)$ is non-empty.

As another consequence of Proposition 5.7, we now state a criterion for topological weak mixing which formally resembles the ergodicity criterion stated as Lemma 4.16 above.

Corollary 5.9. - Let $T \in \mathfrak{B}(X)$. Assume that for each non-empty open subset $\Omega$ of $X$ such that $T^{-1}(\Omega) \subseteq \Omega$ and each neighborhood $W$ of 0 , one can find a minimal $T$-invariant compact set $K$ such that $K \cap \Omega \cap W$ is non-empty. Then $T$ is topologically weakly mixing.

Proof. - By Fact [5.6, every point $z$ in the above compact set $K$ is uniformly recurrent for $T$. We apply Proposition 5.7, taking as $Z$ the set of all uniformly recurrent points for $T$. Let $V$ be a non-empty open subset of $X$. Applying the assumption of Corollary 5.9 to $\Omega:=\bigcup_{n \geq 0} T^{-n}(V)$, we immediately see that the assumption of Proposition 5.7 is satisfied, and hence that $T$ is topologically weakly mixing.

Corollary 5.10. - Let $T \in \mathfrak{B}(X)$, where $X$ is a complex Banach space. Assume that for every $x \in X$ and $\varepsilon>0$, there exist $z \in \operatorname{span} \mathcal{E}(T)$ and $n \geq 0$ such that $\|z\|<\varepsilon$ and $\left\|T^{n} z-x\right\|<\varepsilon$. Then $T$ is topologically weakly mixing and $\operatorname{span} \mathcal{E}(T)$ is dense in $X$.

Proof. - This follows from Fact 5.6 and Proposition 5.7 applied with $Z:=\operatorname{span} \mathcal{E}(T)$.

Our last corollary is the result on which we will elaborate to state our $\mathcal{U}$-frequent hypercyclicity and frequent hypercyclicity criteria.

Corollary 5.11. - Let $T \in \mathfrak{B}(X)$. Assume that for every $x \in X$ and $\varepsilon>0$, there exist $z \in \operatorname{Per}(T)$ and $n \geq 1$ such that $\|z\|<\varepsilon$ and $\left\|T^{n} z-x\right\|<\varepsilon$. Then $T$ is chaotic.

Proof. - Since periodic points are uniformly recurrent, exactly the same proof as that of the previous corollary shows that $T$ is topologically weakly mixing and has a dense set of periodic points. 
Corollary 5.11 can also be proved by a more constructive argument. Since the flexibility of this alternative proof will prove extremely important below for the proofs of some $(\mathcal{U}$-) frequent hypercyclicity criteria, we present it here.

Direct proof of Corollary 5.11. - Before starting the proof, we note that the assumption of Corollary 5.11 obviously implies that $\|T\|>1$ and that $\operatorname{Per}(T)$ is dense in $X$. The first step of the proof is to observe that the assumption of Corollary 5.11 can be reinforced as follows:

Fact 5.12. - Under the assumption of Corollary 5.11, the following property holds true: for any $x \in X$, any $\varepsilon>0$, and any integers $N, M \geq 1$, there exist $z \in \operatorname{Per}(T)$ and $n \geq 1$ with $n=M(\bmod N)$ such that $\|z\|<\varepsilon$ and $\left\|T^{n} z-x\right\|<\varepsilon$.

Proof of Fact 5.12, - Assuming (as we may) that $x$ is non-zero, choose $z^{\prime} \in \operatorname{Per}(T)$ and $n^{\prime} \geq 1$ such that $\left\|z^{\prime}\right\|<\varepsilon^{\prime}$ and $\left\|T^{n^{\prime}} z^{\prime}-x\right\|<\varepsilon$, where $\varepsilon^{\prime}>0$ has to be specified. Then $\|T\|^{n^{\prime}}\left\|z^{\prime}\right\| \geq\|x\|-\varepsilon^{\prime}$, so that $\|T\|^{n^{\prime}} \geq \frac{\|x\|-\varepsilon^{\prime}}{\varepsilon^{\prime}}$. It follows that if $\varepsilon^{\prime}$ is small enough, then $n^{\prime}>N$. Let then $0 \leq r<N$ be an integer such that $n:=n^{\prime}-r$ is equal to $M(\bmod N)$, and set $z=T^{r} z^{\prime}$. Then $\|z\|<\|T\|^{r} \varepsilon^{\prime} \leq\|T\|^{N} \varepsilon^{\prime}$, so $\|z\|<\varepsilon$ if $\varepsilon^{\prime}$ is small enough. Also, $T^{n} z=T^{n^{\prime}-r} T^{r} z^{\prime}=T^{n^{\prime}} z^{\prime}$, so that $\left\|T^{n} z-x\right\|<\varepsilon^{\prime} \leq \varepsilon$.

Let now $\left(x_{j}\right)_{j \geq 1}$ be a dense sequence of vectors of $X$ belonging to $\operatorname{Per}(T)$. A straightforward induction shows that there exists a sequence $\left(z_{j}\right)_{j \geq 1}$ of elements of $\operatorname{Per}(T)$, as well as a strictly increasing sequence $\left(n_{j}\right)_{j \geq 1}$ of integers such that, for every $j \geq 1$,

(i) $\left\|z_{j}\right\|<2^{-j}\|T\|^{-n_{j-1}}$;

(ii) $\left\|T^{n_{j}} z_{j}-\left(x_{j}-\sum_{i<j} z_{i}\right)\right\|<2^{-j}$;

(iii) $n_{j}$ is a multiple of the period of the vector $\sum_{i<j} z_{i}$.

Now, set

$$
z:=\sum_{i=1}^{\infty} z_{i},
$$

which is well-defined by (i). Let us now show that $z$ is a hypercyclic vector for $T$. For every $j \geq 1$, we have $T^{n_{j}}\left(\sum_{i<j} z_{i}\right)=\sum_{i<j} z_{i}$ by (iii), so that

$$
T^{n_{j}} z-x_{j}=\sum_{i<j} z_{i}+T^{n_{j}} z_{j}+\sum_{i>j} T^{n_{j}} z_{i}-x_{j}
$$

It follows that

$$
\begin{aligned}
\left\|T^{n_{j}} z-x_{j}\right\| & \leq\left\|T^{n_{j}} z_{j}-\left(x_{j}-\sum_{i<j} z_{i}\right)\right\|+\sum_{i>j}\|T\|^{n_{j}}\left\|z_{i}\right\| \\
& <2^{-j}+\sum_{i>j}\|T\|^{n_{j}} 2^{-i}\|T\|^{-n_{i-1}} \leq 2^{-(j-1)} \quad \text { by (i) and (ii). }
\end{aligned}
$$

This terminates our direct proof of Corollary [5.11.

To conclude this subsection, we prove a slightly stronger version of Corollary 5.11,

Corollary 5.13. - Let $X_{0}$ be a subset of $X$ with dense linear span. Suppose that for every $x_{0} \in X_{0}$ and every $\varepsilon>0$, there exist $z \in \operatorname{Per}(T)$ and $n \geq 1$ such that $\|z\|<\varepsilon$ and $\left\|T^{n} z-x_{0}\right\|<\varepsilon$. Then $T$ is chaotic. 
Proof. - It is suffices to show that the assumption of Corollary 5.13 can be extended from vectors $x_{0}$ of $X_{0}$ to arbitrary vectors $x$ of $X$ : once this is done, Corollary 5.11 applies.

Since the linear span of $X_{0}$ is dense in $X$, it suffices to consider vectors $x$ of the form $x=\sum_{k=1}^{r} a_{k} x_{k}$, where $x_{k} \in X_{0}$ and $a_{k} \in \mathbb{K} \backslash\{0\}$ for every $1 \leq k \leq r$. An induction on $k, 1 \leq k \leq r$, allows us to construct, using (an obvious modification of) Fact [5.12, vectors $z_{k} \in \operatorname{Per}(T)$ and integers $l_{k}, 1 \leq k \leq r$, with the following properties:

(i) $\left\|z_{k}\right\|<\frac{\varepsilon}{r\left|a_{k}\right|}$;

(ii) $\left\|T^{l_{1}+\cdots+l_{k}} z_{k}-x_{k}\right\|<\frac{\varepsilon}{r\left|a_{k}\right|}$;

(iii) $l_{k}$ is a multiple of $d_{k-1}=\prod_{i=1}^{k-1} \operatorname{per}_{T}\left(z_{i}\right)$.

Now, set $z:=\sum_{k=1}^{r} a_{k} z_{k}$ : this $z$ is clearly a periodic vector for $T$, with $\|z\|<\varepsilon$ by (i). Set also $n:=l_{1}+\cdots+l_{r}$. We have

$$
T^{n} z=\sum_{k=1}^{r} a_{k} T^{n} z_{k}=\sum_{k=1}^{r} a_{k} T^{l_{1}+\cdots+l_{k}} z_{k} \quad \text { by (iii), }
$$

so that

$$
\left\|T^{n} z-x\right\| \leq \sum_{k=1}^{r}\left|a_{k}\right|\left\|T^{l_{1}+\cdots+l_{k}} z_{k}-x_{k}\right\|<\varepsilon \quad \text { by (ii). }
$$

This concludes the proof of Corollary 5.13.

In the next two subsections, we will give two "variations" of Corollary 5.11, where we show that if the assumption $\left\|T^{n} z-x\right\|<\varepsilon$ is replaced by the requirement that the orbit of $z$ approximates that of $x$ during a sufficiently large time, i.e. $\left\|T^{n+k} z-T^{k} x\right\|<\varepsilon$ for a large number of indices $k$, then $T$ is $\mathcal{U}$-frequently hypercyclic, and even sometimes frequently hypercyclic.

5.3. A criterion for $\mathcal{U}$-frequent hypercyclicity. - Here is the version of our criterion for $\mathcal{U}$-frequent hypercyclicity that we will use in Section 6 .

Theorem 5.14. - Let $T \in \mathfrak{B}(X)$. Assume that there exist a dense linear subspace $X_{0}$ of $X$ with $T\left(X_{0}\right) \subseteq X_{0}$ and $X_{0} \subseteq \operatorname{Per}(T)$, and a constant $\alpha \in(0,1)$ such that the following property holds true: for every $x \in X_{0}$ and every $\varepsilon>0$, there exist $z \in X_{0}$ and $n \geq 1$ such that

(1) $\|z\|<\varepsilon$;

(2) $\left\|T^{n+k} z-T^{k} x\right\|<\varepsilon$ for every $0 \leq k \leq n \alpha$.

Then $T$ is chaotic and $\mathcal{U}$-frequently hypercyclic.

Proof. - Since $X_{0}$ is dense in $X$, the periodic points of $T$ are dense in $X$. So we only have to show that $T$ is $\mathcal{U}$-frequently hypercyclic.

As in the direct proof of Corollary 5.11 given above, we first show that given $N \geq 1$, the integer $n$ appearing in the assumption of Theorem 5.14 can be supposed to be a multiple of $N$. Given $x \in X_{0}$ and $\varepsilon>0$, there exist $z^{\prime} \in X_{0}$ and $n^{\prime} \geq 1$ such that $\left\|z^{\prime}\right\|<\varepsilon^{\prime}$ and $\left\|T^{n^{\prime}+k} z^{\prime}-T^{k} x\right\|<\varepsilon^{\prime}$ for every $0 \leq k \leq \alpha n^{\prime}$, where $\varepsilon^{\prime}>0$ has to be specified. Taking $\varepsilon^{\prime}$ small enough, we can assume (see the proof of Fact 5.12) that $n^{\prime}>N$. Let then $0 \leq r<N$ be such that $n:=n^{\prime}-r$ is a multiple of $N$, and set $z=T^{r} z^{\prime}$. Then $z$ belongs to $X_{0}$ 
because $T\left(X_{0}\right) \subseteq X_{0}$. Also $\|z\|<\|T\|^{N} \varepsilon^{\prime}<\varepsilon$ if $\varepsilon^{\prime}$ is small enough; and $T^{n+k} z=T^{n^{\prime}+k} z^{\prime}$, so that $\left\|T^{n+k} z-T^{k} x\right\|<\varepsilon$ for every $0 \leq k \leq \alpha n^{\prime}$. Since $n \leq n^{\prime}$, the inequality holds true a fortiori for every $0 \leq k \leq \alpha$.

Let now $\left(x_{l}\right)_{l \geq 1}$ be a dense sequence of vectors of $X_{0}$, and let $\left(I_{l}\right)_{l \geq 1}$ be a partition of $\mathbb{N}$ into infinite sets. We define a sequence $\left(y_{j}\right)_{j \geq 1}$ of vectors of $X_{0}$ by setting $y_{j}=x_{l}$ for every $j \in I_{l}$. In other words, the sequence $\left(y_{j}\right)_{j \geq 1}$ enumerates infinitely many times each point of the dense sequence $\left(x_{l}\right)_{l \geq 1}$, and $I_{l}$ denotes the set of all indices $j \geq 1$ such that $y_{j}=x_{l}$.

By induction on $j \geq 1$, we construct a sequence $\left(z_{j}\right)_{j \geq 1}$ of vectors of $X_{0}$ and a strictly increasing sequence $\left(n_{j}\right)_{j \geq 1}$ of integers such that

(i) $\left\|T^{k} z_{j}\right\|<2^{-j}$ for every $0 \leq k \leq(1+\alpha) n_{j-1}$;

(ii) $\left\|T^{n_{j}+k} z_{j}-T^{k}\left(y_{j}-\sum_{i<j} z_{i}\right)\right\|<2^{-j}$ for every $0 \leq k \leq \alpha n_{j}$;

(iii) $n_{j}$ is a multiple of the period of the vector $\sum_{i<j} z_{i}$.

We set $z:=\sum_{i>1} z_{i}$, which is well-defined by (i), and prove that $z$ is a $\mathcal{U}$-frequently hypercyclic vector for $T$. Fix $l \geq 1$. For every $j \in I_{l}$ and every $k \geq 0$, we have by (iii)

$$
T^{n_{j}+k} z-x_{l}=T^{k}\left(\sum_{i<j} z_{i}\right)+T^{n_{j}+k} z_{j}-y_{j}+\sum_{i>j} T^{n_{j}+k} z_{i} .
$$

Moreover, if $k$ is a multiple of $\operatorname{per}\left(x_{l}\right)=\operatorname{per}\left(y_{j}\right)$, say $k=m \operatorname{per}\left(x_{l}\right)$, we can write

$$
T^{m \operatorname{per}\left(x_{l}\right)}\left(\sum_{i<j} z_{i}\right)-y_{j}=T^{m \operatorname{per}\left(x_{l}\right)}\left(\sum_{i<j} z_{i}-y_{j}\right) .
$$

Hence we obtain that for any $m \geq 0$,

$$
\begin{gathered}
\left\|T^{n_{j}+m \operatorname{per}\left(x_{l}\right)} z-x_{l}\right\| \leq\left\|T^{n_{j}+m \operatorname{per}\left(x_{l}\right)} z_{j}-T^{m \operatorname{per}\left(x_{l}\right)}\left(y_{j}-\sum_{i<j} z_{i}\right)\right\| \\
+\sum_{i>j}\left\|T^{n_{j}+m \operatorname{per}\left(x_{l}\right)} z_{i}\right\| .
\end{gathered}
$$

By (i) and (ii), it follows that for every $j \geq 1$ and every $0 \leq m \leq \alpha n_{j} / \operatorname{per}\left(x_{l}\right)$, we have

$$
\left\|T^{n_{j}+m \operatorname{per}\left(x_{l}\right)} z-x_{l}\right\|<2^{-j}+\sum_{i>j} 2^{-i}=2^{-(j-1)} .
$$

We deduce from this inequality that for any $\varepsilon>0$,

$$
\begin{aligned}
\overline{\operatorname{dens}} \mathcal{N}_{T}\left(z, B\left(x_{l}, \varepsilon\right)\right) & \geq \limsup _{j \in I_{l}} \frac{1}{(1+\alpha) n_{j}} \#\left\{n_{j}+m \operatorname{per}\left(x_{l}\right) ; 0 \leq m \leq \alpha n_{j} / \operatorname{per}\left(x_{l}\right)\right\} \\
& \geq \frac{\alpha}{(1+\alpha) \operatorname{per}\left(x_{l}\right)}>0 .
\end{aligned}
$$

Hence $z$ is a $\mathcal{U}$-frequently hypercyclic vector for $T$, and Theorem 5.14 follows.

Remark 5.15. - The proof of Theorem 5.14 shows that $T$ admits $\mathcal{U}$-frequently hypercyclic vectors $z \in X$ with the property that for every $l \geq 1$, there exists $\delta_{l}>0$ such that $\overline{\operatorname{dens}} \mathcal{N}_{T}\left(z, B\left(x_{l}, \varepsilon\right)\right) \geq \delta_{l}$ for every $\varepsilon>0$ (the point being that $\delta_{l}$ does not depend on $\varepsilon)$. Hence there exists, for every $l \geq 1$, a sequence $\left(n_{k, l}\right)_{k \geq 1}$ of integers of positive upper density such that $T^{n_{k, l} z}$ tends to $x_{l}$ as $k$ tends to infinity. As $x_{l}$ is a periodic point for $T$, there is no contradiction in this (see [29, Rem. 4.8]). 
5.3.1. Uniform recurrence, almost periodic points and $\mathcal{U}$-frequent hypercyclicity. - Even if this is not quite clear at first sight, the following result generalizes Theorem 5.14 (see Corollary 5.19 below).

Theorem 5.16. - Let $T \in \mathfrak{B}(X)$. Assume that the uniformly recurrent points of $T$ are dense in $X$, and that there exists $\alpha>0$ such that the following property holds true: for any $\varepsilon>0$ and any non-empty open subset $O$ of $X$, one can find $x \in O$ and an arbitrarily large integer $n$ such that $\left\|T^{n+k} x\right\|<\varepsilon$ for every $0 \leq k \leq \alpha$. Then $T$ is $\mathcal{U}$-frequently hypercyclic.

Proof. - It is enough to show that for any non-empty open subset $V$ of $X$, there exists a constant $\alpha_{V}>0$ such that, for every $N \geq 1$, the open set

$$
G_{V, N}:=\left\{u \in X ; \exists m \geq N: \#\left\{i \leq m ; T^{i} u \in V\right\} \geq \alpha_{V} m\right\}
$$

is dense in $X$. Indeed, if $\left(V_{q}\right)_{q \geq 1}$ is a countable basis of open subsets of $X$, the density of each of the sets $G_{V_{q}, N}$ implies that $G:=\bigcap_{N, q} G_{V_{q}, N}$ is a dense $G_{\delta}$ subset of $X$ consisting of $\mathcal{U}$-frequently hypercyclic vectors for $T$.

So let $V$ be a non-empty open subset of $X$. Choose a uniformly recurrent point $v \in V$, and $\varepsilon>0$ such that $B(v, \varepsilon) \subseteq V$. Since $v$ is uniformly recurrent, the set

$$
D_{V}:=\left\{k \geq 1 ;\left\|T^{k} v-v\right\|<\varepsilon / 2\right\}
$$

has bounded gaps. Observe that we may call this set $D_{V}$ since $\varepsilon$ depends on $v$ and $V$, and $v$ depends on $V$. So there exist a constant $c_{V}>0$ and an integer $M_{V} \geq 1$ such that $\#\left(D_{V} \cap J\right) \geq c_{V} \# J$ for all intervals $J$ of $\mathbb{N}$ of length at least $M_{V}$. We set

$$
\alpha_{V}:=\frac{c_{V} \alpha}{1+\alpha}
$$

and we show that with this choice of $\alpha_{V}$, all the open sets $G_{V, N}, N \geq 1$, are dense in $X$. Let us fix $N \geq 1$, and a non-empty open subset $U$ of $X$. We have to show that $G_{V, N} \cap U$ is non-empty. Set $O:=U-v$, which is a non-empty open subset of $X$. By assumption, one can find $x \in O$ and an integer $n \geq N$ such that $\alpha n \geq M_{V}$ and $\left\|T^{n+k} x\right\|<\varepsilon / 2$ for every $0 \leq k \leq \alpha n$. Then $u:=x+v$ belongs to $U$ and our aim is to show that $u$ belongs to $G_{V, N}$. For any $0 \leq k \leq \alpha n$, we have

$$
\left\|T^{n+k} u-v\right\| \leq\left\|T^{n+k} x\right\|+\left\|T^{n+k} v-v\right\|<\varepsilon / 2+\left\|T^{n+k} v-v\right\| ;
$$

in other words,

$$
\forall i \in[n,(1+\alpha) n]:\left\|T^{i} u-v\right\|<\varepsilon / 2+\left\|T^{i} v-v\right\| .
$$

Moreover, since the interval $J=[n,(1+\alpha) n]$ has length at least $M_{V}$, we know that $\#\left(J \cap D_{V}\right) \geq c_{V} \# J$, i.e.

$$
\#\left\{i \in[n,(1+\alpha) n] ;\left\|T^{i} v-v\right\|<\varepsilon / 2\right\} \geq c_{V} \alpha n .
$$

It follows that

$$
\#\left\{1 \leq i \leq(1+\alpha) n ;\left\|T^{i} u-v\right\|<\varepsilon\right\} \geq c_{V} \alpha n,
$$

and hence that $u$ belongs to $G_{V, N}$.

We now state and prove a few consequences of Theorem 5.16. For the first one, we need a definition: if $T$ is a bounded operator on $X$, a point $x \in X$ is said to be almost periodic for $T$ if, for every $\varepsilon>0$, the set

$$
D_{x, \varepsilon}:=\left\{n \geq 1 ; \forall k \geq 1:\left\|T^{n+k} x-T^{k} x\right\|<\varepsilon\right\}
$$


has bounded gaps. Thus, periodic points are almost periodic and almost periodic points are uniformly recurrent. The following fact is more interesting.

Fact 5.17. - Let $T \in \mathfrak{B}(X)$. Then any vector $x$ belonging to span $\mathcal{E}(T)$ is almost periodic for $T$.

Proof. - We first recall that since $x$ belongs to $\operatorname{span} \mathcal{E}(T)$, it is uniformly recurrent for $T$ (Fact 5.6); so for any $\eta>0$, the set $D^{\eta}:=\left\{n \geq 1 ;\left\|T^{n} x-x\right\|<\eta\right\}$ has bounded gaps. Now, assuming that $x$ is non-zero, we write $x$ as $x=\sum_{i=1}^{r} u_{i}$, where $u_{1}, \ldots, u_{r}$ belong to $\mathcal{E}(T)$ and are linearly independent. Then the restriction of $T$ to the finitedimensional subspace $E:=\operatorname{span}\left(u_{1}, \ldots, u_{r}\right)$ is power-bounded, being diagonalizable with only unimodular eigenvalues. Since $E$ contains the $T$-orbit of $x$, it follows that there exists a finite constant $C$ such that, for every $k \geq 0$,

$$
\left\|T^{n+k} x-T^{k} x\right\|=\left\|T^{k}\left(T^{n} x-x\right)\right\| \leq C\left\|T^{n} x-x\right\| .
$$

So the set $D^{\eta}$ with $\eta:=\varepsilon / C$ is contained in $D_{x, \varepsilon}$, which concludes the proof.

From Theorem 5.16, we now deduce

Corollary 5.18. - Let $T \in \mathfrak{B}(X)$. Assume that there exists $\alpha>0$ such that the following property holds true: for every non-empty open subset $O$ of $X$ and every $\varepsilon>0$, there exists an almost periodic point $x \in O$ such that: for any $\eta>0$, one can find $z \in X$ and $n \geq 1$ such that $\|z\|<\eta$ and $\left\|T^{n+k} z-T^{k} x\right\|<\varepsilon$ for all $0 \leq k \leq \alpha$. Then $T$ is $\mathcal{U}$-frequently hypercyclic.

Proof. - By assumption, the uniformly recurrent points are certainly dense in $X$. In order to check the second assumption in Theorem 5.16, let us fix a non-empty open subset $O$ of $X$ and $\varepsilon>0$. Let us choose a non-zero almost periodic point $x_{0} \in O$ satisfying the assumption of Corollary 5.18, and then an integer $M \geq 1$ such that any interval of $\mathbb{N}$ of length $M$ contains a point of the set

$$
D:=\left\{n \geq 1 ; \forall k \geq 1:\left\|T^{n+k} x_{0}-T^{k} x_{0}\right\|<\varepsilon / 2\right\} .
$$

Now, choose $z^{\prime} \in X$ with $\left\|z^{\prime}\right\|$ arbitrarily small and an arbitrarily large integer $n^{\prime}>M$ such that $\left\|T^{n^{\prime}+k} z^{\prime}-T^{k} x_{0}\right\|<\varepsilon / 2$ for every $0 \leq k \leq \alpha n^{\prime}$ (since $x_{0}$ is non-zero, one can ensure that $n^{\prime}$ be arbitrarily large by taking $\left\|z^{\prime}\right\|$ small enough.) Having fixed $z^{\prime}$ and $n^{\prime}$ in this way, we can pick $0 \leq p \leq M$ such that $n:=n^{\prime}-p$ belongs to $D$. Then $z:=T^{p} z^{\prime}$ has arbitrarily small norm, so $x:=x_{0}-z$ belongs to $O$. Moreover, since $n \leq n^{\prime}$ and $T^{n} z=T^{n^{\prime}} z^{\prime}$, we have for every $0 \leq k \leq \alpha n$ :

$$
\left\|T^{n+k} x\right\| \leq\left\|T^{n+k} x_{0}-T^{k} x_{0}\right\|+\left\|T^{k} x_{0}-T^{n^{\prime}+k} z^{\prime}\right\|<\varepsilon / 2+\varepsilon / 2=\varepsilon .
$$

The assumptions of Theorem 5.16] are thus satisfied.

Here is an immediate consequence of Corollary 5.18, whose statement is a bit less convoluted. This result shows in particular that in Theorem [5.14, one can replace the assumption that the vectors of $X_{0}$ are periodic by the assumption that they are almost periodic. Moreover, it also shows that it was in fact unnecessary to assume that $X_{0}$ was a linear subspace of $X$.

Corollary 5.19. - Let $T \in \mathfrak{B}(X)$. Assume that there exist a dense subset $X_{0}$ of $X$ consisting of almost periodic points for $T$, and a constant $\alpha \in(0,1)$ such that the following property holds true: for every $x \in X_{0}$ and every $\varepsilon>0$, there exist $z \in X_{0}$ and $n \geq 1$ such 
that $\|z\|<\varepsilon$ and $\left\|T^{n+k} z-T^{k} x\right\|<\varepsilon$ for all $0 \leq k \leq \alpha$. Then $T$ is $\mathcal{U}$-frequently hypercyclic.

Since Corollary 5.19 is so similar to Theorem 5.14, it is natural to ask whether one can provide a "constructive" proof of it resembling that of Theorem 5.14. We do so now, with the additional assumption that the above dense set $X_{0}$ is a linear subspace of $X$.

Constructive proof of Corollary 5.19 - Define sequences $\left(x_{l}\right)_{l \geq 1},\left(I_{l}\right)_{l \geq 1}$ and $\left(y_{j}\right)_{j \geq 1}$ as in the beginning of the proof of Theorem 5.14. By induction, we construct a sequence $\left(z_{j}\right)_{j \geq 1}$ of vectors of $X_{0}$ and an increasing sequence of integers $\left(n_{j}\right)_{j \geq 1}$ such that

(i) $\left\|T^{k} z_{j}\right\|<2^{-j}$ for every $0 \leq k \leq(1+\alpha) n_{j-1}$;

(ii) $\left\|T^{n_{j}+k} z_{j}-T^{k}\left(y_{j}-\sum_{i<j} z_{i}\right)\right\|<2^{-j}$ for every $0 \leq k \leq \alpha n_{j}$;

(iii') $\left\|T^{n_{j}+k}\left(\sum_{i<j} z_{i}\right)-T^{k}\left(\sum_{i<j} z_{i}\right)\right\|<2^{-j}$ for all $k \geq 0$.

Let $j \geq 1$. Assume that the construction has been carried out up to the step $j-1$, and let us construct $n_{j}$ and $z_{j}$. Since the vector $\sum_{i<j} z_{i}$ lies in $X_{0}$, it is almost periodic; so one can find an integer $M \geq 1$ such that every interval of $\mathbb{N}$ of length $M$ contains a point of the set

$$
D:=\left\{n \geq 1 ; \forall k \geq 1:\left\|T^{n+k}\left(\sum_{i<j} z_{i}\right)-T^{k}\left(\sum_{i<j} z_{i}\right)\right\|<2^{-j}\right\} .
$$

By assumption on $T$, there exists $n^{\prime} \in \mathbb{N}$ with $n^{\prime}>n_{j-1}+M$ and $z^{\prime} \in X_{0}$ such that

- $\left\|T^{k} z^{\prime}\right\|<2^{-j}$ for every $0 \leq k \leq(1+\alpha) n_{j-1}+M$;

- $\left\|T^{n^{\prime}+k} z^{\prime}-T^{k}\left(y_{j}-\sum_{i<j} z_{i}\right)\right\|<2^{-j}$ for every $0 \leq k \leq \alpha n^{\prime}$.

Choose now $0 \leq p \leq M$ such that $n_{j}:=n^{\prime}-p$ belongs to the set $D$, and let $z_{j}:=T^{p} z^{\prime}$. Then (iii') holds true by the choice of $n_{j}$, (i) clearly holds true, and (ii) holds true as well because $T^{n_{j}+k} z_{j}=T^{n^{\prime}+k} z^{\prime}$ for every $k \geq 0$ and every $n^{\prime} \geq n_{j}$. This concludes the inductive step.

Let us prove that the vector $z:=\sum_{i>1} z_{i}$ is a $\mathcal{U}$-frequently hypercyclic vector for $T$. This time, we write for every $l \geq 1, j \in I_{l}$ and $k \geq 0$ :

$$
\begin{aligned}
T^{n_{j}+k} z-x_{l}= & T^{n_{j}+k}\left(\sum_{i<j} z_{i}\right)-T^{k}\left(\sum_{i<j} z_{i}\right)+T^{n_{j}+k} z_{j}-T^{k}\left(y_{j}-\sum_{i<j} z_{i}\right) \\
& +T^{k} x_{l}-x_{l}+\sum_{i>j} T^{n_{j}+k} z_{i} .
\end{aligned}
$$

Since $n_{j}$ belongs to $D$, we deduce that if $0 \leq k \leq \alpha n_{j}$, then

$$
\left\|T^{n_{j}+k} z-x_{l}\right\| \leq 2^{-j}+2^{-j}+\left\|T^{k} x_{l}-x_{l}\right\|+2^{-j}=3 \cdot 2^{-j}+\left\|T^{k} x_{l}-x_{l}\right\| .
$$

Now, since $x_{l}$ is uniformly recurrent for $T$, the set

$$
D_{l, \varepsilon}:=\left\{k \geq 1 ;\left\|T^{k} x_{l}-x_{l}\right\|<\varepsilon / 2\right\}
$$

has bounded gaps. So one can find a constant $c_{l, \varepsilon}>0$ such that $\#\left(D_{l, \varepsilon} \cap J\right) \geq c_{l, \varepsilon} \# J$ for all sufficiently large intervals $J \subseteq \mathbb{N}$. From this, it follows that if $j$ is large enough, then

$$
\#\left\{n_{j} \leq i \leq(1+\alpha) n_{j} ;\left\|T^{i} z-x_{l}\right\|<\varepsilon\right\} \geq c_{l, \varepsilon} \alpha n_{j},
$$

and hence that

$$
\overline{\operatorname{dens}} \mathcal{N}_{T}\left(z, B\left(x_{l}, \varepsilon\right)\right) \geq c_{l, \varepsilon} \limsup _{j \rightarrow \infty} \frac{\alpha n_{j}}{(1+\alpha) n_{j}}=\frac{c_{l, \varepsilon} \alpha}{1+\alpha}
$$


Finally, here is a somewhat unexpected consequence of Theorem 5.16 ,

Corollary 5.20. - Let $T \in \mathfrak{B}(X)$. Assume that the uniformly recurrent points of $T$ are dense in $X$, and that there also exists a dense set of points $x \in X$ such that $T^{i} x \rightarrow 0$ as $i \rightarrow \infty$. Then $T$ is $\mathcal{U}$-frequently hypercyclic.

Proof. - This follows immediately from Theorem 5.16,

Remark 5.21. - Corollary 5.20 shows in particular that if a unilateral weighted backward shift on $\ell^{p}(\mathbb{N})$ or $c_{0}(\mathbb{N})$ has a dense set of uniformly recurrent points, then it is $\mathcal{U}$-frequently hypercyclic. However, a much stronger result is true: if a weighted backward shift has just a single non-zero uniformly recurrent point, then it is in fact chaotic and frequently hypercyclic.

Proof. - Let $B_{\boldsymbol{\omega}}$ be a weighted backward shift on $X=c_{0}(\mathbb{N})$ or $\ell_{p}(\mathbb{N}), 1 \leq p<\infty$, associated to the weight sequence $\boldsymbol{\omega}=\left(\omega_{k}\right)_{k \geq 1}$, and assume that $B_{\boldsymbol{\omega}}$ has a non-zero uniformly recurrent point $x \in X$. By the classical Frequent Hypercyclicity/Chaoticity Criterion (see [7] or [31]), it is enough to show that the sequence $\left(\prod_{k=1}^{n} \omega_{k}^{-1}\right)$ belongs to the space $X$. We check this in the case where $X=c_{0}(\mathbb{N})$, the $\ell_{p}$ case being similar. So we have to show that $\prod_{k=1}^{n} \omega_{k} \rightarrow \infty$ as $n \rightarrow \infty$. Since $x=\sum_{k \geq 1} x_{k} e_{k}$ is non-zero, there exists $k_{0} \geq 1$ such that $\left|x_{k_{0}}\right|>0$. Let $0<\varepsilon<\left|x_{k_{0}}\right| / 2$. Since $x$ is uniformly recurrent for $B_{\boldsymbol{\omega}}$, there exists a strictly increasing sequence of integers $\left(n_{j}\right)_{j \geq 1}$ and a positive integer $M$ such that $n_{j+1}-n_{j} \leq M$ and $\left\|B_{\omega}^{n_{j}} x-x\right\|<\varepsilon$ for every $j \geq 1$. In particular, we have

$$
\left|\left(\prod_{k=k_{0}+1}^{k_{0}+n_{j}} \omega_{k}\right) x_{k_{0}+n_{j}}-x_{k_{0}}\right|<\varepsilon
$$

and hence

$$
\prod_{k=k_{0}+1}^{k_{0}+n_{j}}\left|\omega_{k}\right|>\frac{\varepsilon}{\left|x_{k_{0}+n_{j}}\right|} .
$$

Since $x$ belongs to $c_{0}(\mathbb{N})$, we deduce that

$$
\prod_{k=k_{0}+1}^{k_{0}+n_{j}}\left|\omega_{k}\right| \rightarrow \infty \quad \text { as } j \rightarrow \infty .
$$

Moreover, if $n$ is a sufficiently large integer, there exists an integer $j \geq 1$ such that $k_{0}+n_{j-1} \leq n<k_{0}+n_{j}$. Since $n_{j+1}-n_{j} \leq M$, this implies that

$$
\prod_{k=k_{0}+1}^{n}\left|\omega_{k}\right| \geq \frac{\prod_{k=k_{0}+1}^{k_{0}+n_{j}}\left|\omega_{k}\right|}{\|w\|_{\infty}^{M}}
$$

and this concludes the proof.

Remark 5.22. - A natural question that comes to mind in view of Corollary 5.20 is whether any chaotic and topologically mixing operator has to be $\mathcal{U}$-frequently hypercyclic. We will prove in Section 6.8 that it is not the case. 
5.3.2. More about $\mathcal{U}$-frequent hypercyclicity and $c(T)$. - Corollary 5.20 says in essence that lots of uniformly recurrent points plus lots of orbits tending to 0 imply $\mathcal{U}$-frequent hypercyclicity. In the same spirit, we now prove the following result, which again may look rather surprising at first sight.

Theorem 5.23. - Let $T \in \mathfrak{B}(X)$ be hypercyclic, and assume that $T$ has a dense set of uniformly recurrent points. Then $T$ is $\mathcal{U}$-frequently hypercyclic if and only if $c(T)>0$.

Proof. - One implication is clear: if $T$ is $\mathcal{U}$-frequently hypercyclic, then $c(T)>0$ by the very definition of $c(T)$.

Conversely, assume that $c(T)>0$. Then there is a comeager set $G \subseteq X$ such that, for every $x \in G$, one can find a subset $D_{x}$ of $\mathbb{N}$ with $\overline{\operatorname{dens}} D_{x} \geq c(T)$ such that $\left\|T^{n} x\right\| \rightarrow 0$ as $n \rightarrow \infty$ along $D_{x}$ (see [29, Prop. 4.7]). The key step of the proof lies in the next fact, where we use for convenience the following notation: if $V$ is an open subset of $X$ and $B=B(u, \varepsilon)$ is an open ball of $X$, we write $B \prec V$ if there exists $\varepsilon^{\prime}>\varepsilon$ such that $B\left(u, \varepsilon^{\prime}\right) \subseteq V$.

Fact 5.24. - Let $x_{0} \in X$ be a uniformly recurrent point for $T$, and let $V$ be an open neighbourhood of $x_{0}$. Let also $B$ be an open ball with center $x_{0}$ such that $B \prec V$, and choose an integer $N$ such that every interval $I \subseteq \mathbb{N}$ of cardinality at least $N$ intersects $\mathcal{N}_{T}\left(x_{0}, B\right)$. Then, for every $y \in x_{0}+G$, we have $\overline{\operatorname{dens}} \mathcal{N}_{T}(y, V) \geq c(T) / N$.

Proof of Fact 5.24 - Write $y=x_{0}+x$, with $x \in G$. Since $\overline{\operatorname{dens}} D_{x} \geq c(T)$ and $\bigcup_{k=0}^{N-1}\left(\mathcal{N}_{T}\left(x_{0}, B\right)-k\right)=\mathbb{N}$ by the choice of $N$, one can find $k \in\{0, \ldots, N-1\}$ such that $\overline{\operatorname{dens}}\left(D_{x} \cap\left(\mathcal{N}_{T}\left(x_{0}, B\right)-k\right)\right) \geq c(T) / N$; equivalently, $\overline{\operatorname{dens}}\left(\left(D_{x}+k\right) \cap \mathcal{N}_{T}\left(x_{0}, B\right)\right) \geq$ $c(T) / N$. Now, we have $T^{n} y=T^{n} x_{0}+T^{n} x$ for all $n \in \mathbb{N}$, and $T^{n} x \rightarrow 0$ as $n \rightarrow \infty$ along $D_{x}+k$. Since $B \prec V$, it follows that all but finitely many integers $n \in\left(D_{x}+k\right) \cap \mathcal{N}_{T}\left(x_{0}, B\right)$ belong to $\mathcal{N}_{T}(y, V)$, and hence that $\mathcal{N}_{T}(y, V) \geq c(T) / N$.

It is now easy to conclude the proof of Theorem 5.23. Let $\left(V_{p}\right)_{p \geq 1}$ be a countable basis of (non-empty) open sets for $X$. Choosing for each $p \geq 1$ a uniformly recurrent point $x_{p} \in V_{p}$ and an open ball $B_{p}$ with center $x_{p}$ such that $B_{p} \prec V_{p}$, we see that the following holds true: for each $p \geq 1$, there is an integer $N_{p}$ and a comeager set $G_{p} \subseteq X$ (namely, $\left.G_{p}=x_{p}+G\right)$ such that $\overline{\operatorname{dens}} \mathcal{N}_{T}\left(z, V_{p}\right) \geq c(T) / N_{p}$ for every $z \in G_{p}$. Then every vector $z$ in the comeager set $G_{\infty}:=\bigcap_{p \geq 1} G_{p}$ is a $\mathcal{U}$-frequently hypercyclic vector for $T$.

Remark 5.25. - In Theorem 5.23, one cannot replace the assumption of $\mathcal{U}$-frequent hypercyclicity with that of frequent hypercyclicity. Indeed, as we shall see in Theorem6.29, there exist operators which are chaotic and $\mathcal{U}$-frequently hypercyclic (hence, hypercyclic with a dense set of uniformly recurrent points and such that $c(T)>0$ ) but not frequently hypercyclic.

When the operator $T$ is chaotic, the proof of Theorem 5.23 gives a more precise statement, which says that if $c(T)>0$, then $T$ is " $\mathcal{U}$-frequently hypercyclic with estimates".

Corollary 5.26. - Let $T$ be a chaotic operator on $X$ with $c(T)>0$. For any open set $V \neq \emptyset$ in $X$, let us denote by $N(V)$ the smallest period of all periodic vectors of $T$ belonging to $V$. Then there is a comeager set of vectors $z \in X$ such that

$$
\overline{\operatorname{dens}} \mathcal{N}_{T}(z, V) \geq \frac{1}{N(V)} c(T) \quad \text { for every open set } V \neq \emptyset \text {. }
$$


Proof. - Note that if the point $x_{0}$ in Fact 5.24 is a periodic point of $T$, then one can take as $N$ the period of $x_{0}$. Then follow the proof of Theorem 5.23 .

From Corollary 5.26, we immediately deduce

Corollary 5.27. - If $T \in \mathfrak{B}(X)$ is chaotic and ergodic, then there is a comeager set of vectors $z \in X$ such that $\overline{\text { dens }} \mathcal{N}_{T}(z, V) \geq 1 / N(V)$ for every non-empty open subset $V$ of $X$.

Proof. - This is clear since $c(T)=1$ for any ergodic operator $T$.

Remark 5.28. - All vectors $z \in X$ satisfying the conclusion of Corollary 5.27 are $\mathcal{U}$ frequently hypercyclic for $T$, but none of them is frequently hypercyclic. Indeed, if $V_{0}$ is any open ball centered at 0 , then $N\left(V_{0}\right)=1$, so that $\overline{\operatorname{dens}} \mathcal{N}_{T}\left(z, V_{0}\right)=1$. Hence, one must have dens $\mathcal{N}_{T}(z, V)=0$ for any open set $V$ disjoint from $V_{0}$.

To conclude this section, we now proceed to prove a generalization of Corollary 5.27 to ergodic operators $T \in \mathfrak{B}(X)$ which are not necessarily chaotic: we require that $T$ belongs to $\operatorname{SPAN}(X)$, i.e. that the unimodular eigenvectors of $T$ span a dense subspace of $X$.

We first need to introduce some notation. To every $\lambda \in \mathbb{T}$, we associate the rotationinvariant measure $\nu_{\lambda}$ on $\mathbb{T}$ defined as follows:

$$
\nu_{\lambda}=\left\{\begin{array}{l}
\frac{1}{N} \sum_{k=0}^{N-1} \delta_{\left\{\lambda^{k}\right\}} \quad \text { if } \lambda^{N}=1 \text { for some } N \geq 1 \\
\text { the normalized Lebesgue measure on } \mathbb{T} \text { otherwise. }
\end{array}\right.
$$

Suppose now that $T \in \mathfrak{B}(X)$, and that $\mathbf{u}=\left(u_{1}, u_{2}, \ldots\right)$ is a finite or infinite sequence of linearly independent eigenvectors associated to unimodular eigenvalues $\lambda_{1}, \lambda_{2}, \ldots$ Let us denote by $\mathcal{E}^{\mathbf{u}}$ the linear span of the vectors $u_{j}, j \geq 1$. Any vector $u \in \mathcal{E}^{\mathbf{u}}$ may be written in a unique way as

$$
u=\sum_{k=1}^{r} a_{k}^{\mathbf{u}}(u) u_{k}
$$

where the coefficients $a_{k}^{\mathbf{u}}(u), 1 \leq k \leq r$, are complex scalars. To any such vector $u$, we associate the $T$-invariant measure $\nu_{u, T}^{\mathbf{u}}$ on $X$ defined by

$$
\nu_{u, T}^{\mathbf{u}}(A)=\prod_{k=1}^{r} \nu_{\lambda_{k}}\left(\left\{\left(\mu_{1}, \ldots, \mu_{r}\right) \in \mathbb{T}^{r} ; \sum_{k=1}^{r} a_{k}^{\mathbf{u}}(u) \mu_{k} u_{k} \in A\right\}\right)
$$

for every Borel subset $A$ of $X$. Note that if the unimodular numbers $\lambda_{k}$ are not roots of unity (and $X$ is a Hilbert space $\mathcal{H}$ ), then $\nu_{u, T}^{\mathbf{u}}$ is nothing but the Steinhaus measure associated to $u$ considered in the proof of Fact 4.17 .

Finally, for any open set $V \neq \emptyset$, we define

$$
\delta_{V, T}^{\mathbf{u}}:=\sup \left\{\nu_{u, T}^{\mathbf{u}}(B(u, \varepsilon)) ; \varepsilon>0, u \in \mathcal{E}^{\mathbf{u}}, B(u, \varepsilon) \prec V\right\} .
$$

As in Fact [5.24, the notation $B(u, \varepsilon) \prec V$ means that $B\left(u, \varepsilon^{\prime}\right) \subseteq V$ for some $\varepsilon^{\prime}>\varepsilon$.

We may now state 
Proposition 5.29. - Let $T$ be an ergodic operator on $X$ belonging to $\operatorname{SPAN}(X)$, and let $\mathbf{u}=\left(u_{k}\right)_{k \geq 1}$ be an infinite linearly independent sequence of unimodular eigenvectors whose linear span $\mathcal{E}^{\mathbf{u}}$ is dense in $X$. Then, there exists a comeager subset $G$ of $X$ such that every vector $z \in G$ satisfies

$$
\overline{\operatorname{dens}} \mathcal{N}_{T}(z, V) \geq \delta_{V, T}^{\mathrm{u}} \quad \text { for every open set } V \neq \emptyset \text {. }
$$

The key step in the proof of Proposition 5.29 is Lemma 5.30 below.

Lemma 5.30. - Let $T$ be an ergodic operator on $X$, and let $\mathbf{u}=\left(u_{1}, \ldots, u_{r}\right)$ be a finite sequence of linearly independent unimodular eigenvectors for $T$. Let also $u$ be a vector belonging to $\mathcal{E}^{\mathbf{u}}$. Given any $\varepsilon, \gamma>0$, there exists a $T$-invariant measure $m$ on $X$, ergodic for $T$ and with full support, such that

$$
m(B(u, \varepsilon)) \geq(1-\gamma) \nu_{u, T}^{\mathbf{u}}(B(u,(1-\gamma) \varepsilon)) .
$$

Since the sequence $\mathbf{u}$ is fixed, we will remove any reference to it in the proofs of Proposition 5.29 and Lemma 5.30, so we will write for instance $a_{k}(u)$ instead of $a_{k}^{\mathbf{u}}(u)$, and so on.

Proof of Lemma 5.30 - Let $m_{0}$ be an ergodic measure with full support for T. For each $R>0$, consider the probability measure $m_{R}$ defined on $X$ by setting

$$
m_{R}(A)=\int_{K_{u}} m_{0}(R(A-x)) d \nu_{u, T}(x) \text { for every Borel subset } A \text { of } X,
$$

where $K_{u}:=\left\{\sum_{k=1}^{r} a_{k}(u) \mu_{k} u_{k}, \mu_{k} \in \mathbb{T}, 1 \leq k \leq r\right\}$. Note that $K_{u}$ is a compact subset of $X$ and contains the support of the measure $\nu_{u, T}$. It clearly satisfies $T\left(K_{u}\right)=K_{u}$. The measure $m_{R}$ thus defined is $T$-invariant. Indeed, we have for every Borel subset $A$ of $X$

$$
\begin{aligned}
m_{R}\left(T^{-1} A\right) & =\int_{K_{u}} m_{0}\left(R\left(T^{-1} A-x\right)\right) d \nu_{u, T}(x)=\int_{K_{u}} m_{0}\left(R\left(T^{-1}(A-T x)\right)\right) d \nu_{u, T}(x) \\
& =\int_{T\left(K_{u}\right)} m_{0}(R(A-x)) d \nu_{u, T}(x)=m_{R}(A) .
\end{aligned}
$$

The next step of the proof is to show that $m_{R}(\mathrm{HC}(T))=1$. Since

$$
m_{R}(\mathrm{HC}(T))=\int_{K_{u}} m_{0}(R(\mathrm{HC}(T)-x)) d \nu_{u, T}(x)
$$

and $m_{0}(\mathrm{HC}(T))=1$, it suffices to show that $\mathrm{HC}(T) \subseteq \mathrm{HC}(T)-x$ for every vector $x \in K_{u}$, i.e. that $\mathrm{HC}(T)+x \subseteq \operatorname{HC}(T)$ for every such $x$. Write $x$ as $x=\sum_{k=1}^{r} a_{k} \mu_{k} u_{k}$, where $a_{k}=a_{k}(u)$ and $\mu_{k}$ belongs to $\mathbb{T}$ for every $1 \leq k \leq r$, and fix a vector $z \in \operatorname{HC}(T)$. In order to show that $z+x$ belongs to $\operatorname{HC}(T)$, we need to show that for every $y \in X$ and every $\delta>0$, there exists an integer $n \geq 1$ such that $\left\|T^{n}(z+x)-y\right\|<\delta$. Now, a result of Shkarin [47] states the following: given a hypercyclic operator $S$ on a Banach space $Z$, and a compact topological group $G$ generated by an element $g \in G$, the set

$$
\left\{\left(S^{n} z, g^{n}\right), n \geq 1\right\}
$$

is dense in $Z \times G$ for every vector $z \in Z$ which is hypercyclic for $S$. We apply this result to the operator $T$ and to the subgroup $G$ of $\mathbb{T}^{r}$ generated by $g:=\left(\lambda_{1}, \ldots, \lambda_{r}\right)$. Since the $r$ tuple $(1, \ldots, 1)$ belongs to $G$, and $z \in X$ is a hypercyclic vector for $T$, there exists for every 
$\delta>0$ an integer $n \geq 1$ such that $\left\|T^{n} z-(y-x)\right\|<\delta / 2$ and $\max _{1 \leq k \leq r}\left|a_{k}\right| \cdot\left\|u_{k}\right\| \cdot\left|\lambda_{k}^{n}-1\right|<$ $\delta /(2 r)$. It follows that

$$
\left\|T^{n} x-x\right\|=\left\|\sum_{k=1}^{r} a_{k}\left(\lambda_{k}^{n}-1\right) u_{k}\right\|<\frac{\delta}{2},
$$

so that $\left\|T^{n}(z+x)-y\right\|<\delta$. This proves our claim, and shows that $m_{R}(\mathrm{HC}(T))=1$ for every $R>0$.

Let us now estimate from below the quantities $m_{R}(B(u, \varepsilon))$. By the definition of $m_{R}$, we have

$$
m_{R}(B(u, \varepsilon)) \geq \int_{K_{u} \cap B(u,(1-\gamma) \varepsilon)} m_{0}(R(B(u, \varepsilon)-x)) d \nu_{u, T}(x) .
$$

Now, observe that for every $x \in B(u,(1-\gamma) \varepsilon)$, the set $B(u, \varepsilon)-x$ contains the ball $B(0, \gamma \varepsilon)$. Indeed, every $y \in X$ with $\|y\|<\gamma \varepsilon$ can be written as $y=u+(y-u+x)-x$ with $\|y-u+x\|<\gamma \varepsilon+(1-\gamma) \varepsilon=\varepsilon$. It follows that

$$
\begin{aligned}
m_{R}(B(u, \varepsilon)) & \geq \int_{K_{u} \cap B(0,(1-\gamma) \varepsilon)} m_{0}(B(0, R \gamma \varepsilon)) d \nu_{u, T}(x) \\
& =m_{0}(B(0, R \gamma \varepsilon)) \cdot \nu_{u, T}(B(u,(1-\gamma) \varepsilon)) .
\end{aligned}
$$

Since $m_{0}(B(0, R \gamma \varepsilon))$ tends to 1 as $R$ tends to infinity, there exists $R_{0}>0$ such that

$$
m_{R_{0}}(\mathrm{HC}(T) \cap B(u, \varepsilon))=m_{R_{0}}(B(u, \varepsilon))>(1-\gamma) \nu_{u, T}(B(u,(1-\gamma) \varepsilon)) .
$$

Applying the Ergodic Decomposition Theorem (see e.g. [45, Th. 2.5]) to the measure $m_{R_{0}}$, we obtain that there exists an ergodic measure $m$ for $T$ such that

$$
m(\mathrm{HC}(T) \cap B(u, \varepsilon))>(1-\gamma) \nu_{u, T}(B(u,(1-\gamma) \varepsilon)) .
$$

Since $m(\operatorname{HC}(T))>0$, the measure $m$ has full support, and this concludes the proof of Lemma 5.30 .

Proof of Proposition 5.29, - Let $\left(V_{p}\right)_{p>1}$ be a countable basis of (non-empty) open sets for $X$ with the following property: for any open set $V$ and any open ball $B$ such that $B \prec V$, one can find $p \geq 1$ such that $B \prec V_{p} \subseteq V$. This additional property implies that for any open set $V \neq \emptyset$, we have

$$
\delta_{V, T}=\sup \left\{\delta_{V_{p}, T} ; V_{p} \subseteq V\right\} .
$$

For each $p \geq 1$, one may choose a sequence $\left(u_{p, k}\right)_{k \geq 1}$ of vectors of $\mathcal{E}^{\mathbf{u}}$, a sequence of positive numbers $\left(\varepsilon_{p, k}\right)_{k \geq 1}$ and a sequence of positive numbers $\left(\gamma_{p, k}\right)_{k \geq 1}$ tending to 0 as $k$ tends to infinity such that

$B\left(u_{p, k}, \varepsilon_{p, k}\right) \prec V_{p} \quad$ for every $k \geq 1$ and $\alpha_{p, k}:=\nu_{u_{p, k}, T}\left(B\left(u_{p, k},\left(1-\gamma_{p, k}\right) \varepsilon_{p, k}\right)\right) \underset{k \rightarrow \infty}{\longrightarrow} \delta_{V_{p}, T}$.

By Lemma 5.30 and the pointwise ergodic theorem, we see that for each fixed pair $(p, k)$ of integers, the set of all vectors $z \in X$ such that

$$
\underline{\operatorname{dens}} \mathcal{N}_{T}\left(z, B\left(u_{p, k}, \varepsilon_{p, k}\right)\right) \geq\left(1-\gamma_{p, k}\right) \alpha_{p, k}
$$

is dense in $X$. In particular, the set

$$
G_{p, k}:=\bigcap_{N \geq 1} \bigcup_{n \geq N}\left\{z \in X ; \#\left\{1 \leq i \leq n ; T^{i} z \in B\left(u_{p, k}, \varepsilon_{p, k}\right)\right\} \geq\left(1-\gamma_{p, k}\right) \alpha_{p, k}\right\}
$$


is a dense $G_{\delta}$ subset of $X$. Hence $G:=\bigcap_{p, k \geq 1} G_{p, k}$ is a dense $G_{\delta}$ subset of $X$ too, and by the definition of $G$, every vector $z \in G$ satisfies

$$
\overline{\operatorname{dens}} \mathcal{N}_{T}\left(z, V_{p}\right) \geq \delta_{V_{p}, T} \quad \text { for every } p \geq 1 .
$$

Property (15) then allows us to conclude the proof of Proposition 5.29.

5.4. A criterion for frequent hypercyclicity. - We now move over to a criterion of the same kind as Theorem 5.14 for frequent hypercyclicity. Instead of requiring in the assumption that $\left\|T^{n+k} z-T^{k} x\right\|$ be small for indices $k$ less than a fraction of $n$, we have to require that these quantities should be small for indices $k$ less than a fraction of a certain multiple $d$ of the period of $z$. The criterion reads as follows:

Theorem 5.31. - Let $T \in \mathfrak{B}(X)$. Assume that there exist a dense linear subspace $X_{0}$ of $X$ with $T\left(X_{0}\right) \subseteq X_{0}$ and $X_{0} \subseteq \operatorname{Per}(T)$, and a constant $\alpha \in(0,1)$ such that the following property holds true: for every $x \in X_{0}$, every $\varepsilon>0$ and every integer $d_{0} \geq 1$ which is the period of some vector $y$ of $X_{0}$, there exist $z \in X_{0}$ and integers $n, d \geq 1$ such that

(0) $d$ is a multiple of $d_{0}$ and of $\operatorname{per}(z)$;

(1) $\left\|T^{k} z\right\|<\varepsilon$ for every $0 \leq k \leq \alpha d$;

(2) $\left\|T^{n+k} z-T^{k} x\right\|<\varepsilon$ for every $0 \leq k \leq \alpha d$.

Then $T$ is chaotic and frequently hypercyclic.

Remark 5.32. - The restriction that the integer $d_{0}$ above should be the period of some vector $y \in X_{0}$ may seem rather artificial. The reason for stating Theorem 5.31 as we did is to be found in the proof of Theorem 6.9 below: we will use Theorem 5.31 as stated to prove the frequent hypercyclicity of the operators involved there without having to impose certain divisibility conditions on the numbers $\Delta^{(k)}, k \geq 1$.

Remark 5.33. - The constant $\alpha$ involved in the statement of Theorem 5.31 cannot be greater than $1 / 2$. Indeed, otherwise one could find a periodic vector $z$ such that more than half of the points in the orbit of $z$ are close to 0 and more than half of these points are close to some periodic orbit far away from 0 . So at least one point in the orbit of $z$ would have to be both close to 0 and far away from 0 , which is impossible.

Proof of Theorem 5.31. - We first note that upon substracting some multiple of $d$ to $n$ (where $d$ and $n$ are given by the assumptions of Theorem [5.31), we can always assume that $d \geq n$. The assumption of Theorem 5.31 is thus seen to be stronger than that of Theorem 5.14. Also, we can require that $n>\alpha d$ and the same argument as in the beginning of the proof of Theorem 5.14 (taking $\varepsilon$ very small) shows that, given $N \geq 1$, we can always add to the assumption of Theorem 5.31 the additional hypothesis that the integer $n$ is a multiple of $N$.

Let now $\left(x_{l}\right)_{l \geq 1}$ be a dense sequence of vectors of $X$ contained in $X_{0}$, and let $\left(I_{l}\right)_{l \geq 1}$ be a partition of $\mathbb{N}$ such that each set $I_{l}$ is infinite and has bounded gaps. We denote by $r_{l}$ the maximum size of a gap between two successive elements of $I_{l}$. As usual, we define the vectors $y_{j}, j \geq 1$, by setting $y_{j}=x_{l}$ for every $j \in I_{l}$. By induction on $j \geq 1$, we construct a sequence $\left(z_{j}\right)_{j \geq 1}$ of vectors of $X_{0}$ and two strictly increasing sequences of integers $\left(d_{j}\right)_{j \geq 1}$ and $\left(n_{j}\right)_{j \geq 1}$ such that

(i) $d_{j}$ is a multiple of per $\left(\sum_{i=1}^{j-1} z_{i}\right)$ and of $\operatorname{per}\left(z_{j}\right)$;

(ii) $\left\|T^{k} z_{j}\right\|<2^{-j}$ for every $0 \leq k \leq \alpha d_{j}$;

(iii) $\left\|T^{n_{j}+k} z_{j}-T^{k}\left(y_{j}-\sum_{i=1}^{j-1} z_{i}\right)\right\|<2^{-j}$ for every $0 \leq k \leq \alpha d_{j}$; 
(iv) $n_{j}$ is a multiple of $\operatorname{per}\left(\sum_{i=1}^{j-1} z_{i}\right)$ and $\alpha d_{j}<n_{j} \leq d_{j}$;

(v) $\alpha d_{j}>4 d_{j-1}$.

We set $x=\sum_{i \geq 1} z_{i}$, and show that $z$ is a frequently hypercyclic vector for $T$.

Let us fix $l \geq 1$, and write $I_{l}$ as $I_{l}=\left\{j_{m} ; m \geq 1\right\}$, where $\left(j_{m}\right)_{m \geq 1}$ is strictly increasing and $j_{m+1}-j_{m} \leq r_{l}$ for every $m \geq 1$. For each $m \geq 1$, we define a family of sets $\left(A_{m, j}\right)_{0 \leq j<j_{m+1}-j_{m}}$ as follows:

$$
A_{m, 0}:=\left\{n_{j_{m}}+k d_{j_{m}}+k^{\prime} \operatorname{per}\left(x_{l}\right) ; 0 \leq k^{\prime} \leq \frac{\alpha d_{j_{m}}}{\operatorname{per}\left(x_{l}\right)}, 0 \leq k \leq \frac{\alpha d_{j_{m}+1}}{d_{j_{m}}}-2\right\},
$$

and, for $1 \leq j<j_{m+1}-j_{m}$,

$$
A_{m, j}:=\bigcup_{1 \leq k \leq \frac{\alpha d_{j_{m}+j+1}}{d_{j_{m}+j}}-1}\left(A_{m, j-1}+k d_{j_{m}+j}\right) .
$$

Before doing anything with these sets, we note that

$$
\max A_{m, j} \leq \alpha d_{j_{m}+j+1} .
$$

Indeed, for $j=0$ we have $\max A_{m, 0} \leq n_{j_{m}}+\alpha d_{j_{m}+1}-2 d_{j_{m}}+\alpha d_{j_{m}} \leq \alpha d_{j_{m}+1}$ because $n_{j_{m}} \leq d_{j_{m}}$; and the result then follows by a straightforward induction on $j<j_{m+1}-j_{m}$.

Fact 5.34. - For every $m \geq 1$ and every $n \in A_{m, j}, 0 \leq j<j_{m+1}-j_{m}$, we have

$$
\left\|T^{n} z-x_{l}\right\| \leq 2^{-\left(j_{m}-1\right)} .
$$

Proof of Fact5.34 - For any $n \in A_{m, j}$, we have

$$
\left\|T^{n} z-x_{l}\right\| \leq\left\|T^{n}\left(\sum_{s=1}^{j_{m}+j} z_{s}\right)-x_{l}\right\|+\sum_{s>j_{m}+j}\left\|T^{n} z_{s}\right\| .
$$

Since $n \leq \alpha d_{j_{m}+j+1} \leq \alpha d_{s}$ for every $s>j_{m}+j$ by (16), the second term in (17) is easy to control:

$$
\sum_{s>j_{m}+j}\left\|T^{n} z_{s}\right\|<\sum_{s>j_{m}+j} 2^{-s}
$$

We now have to estimate the term $\left\|T^{n}\left(\sum_{s=1}^{j_{m}+j} z_{s}\right)-x_{l}\right\|$. The index $m$ being fixed, we show by induction on $0 \leq j<j_{m+1}-j_{m}$ that

$$
\left\|T^{n}\left(\sum_{s=1}^{j_{m}+j} z_{s}\right)-x_{l}\right\|<\sum_{u=0}^{j} 2^{-\left(j_{m}+u\right)} .
$$

- Suppose first that $n$ belongs to $A_{m, 0}$, so that

$$
n=n_{j_{m}}+k d_{j_{m}}+k^{\prime} \operatorname{per}\left(x_{l}\right) \quad \text { with } \quad 0 \leq k \leq \frac{\alpha d_{j_{m}+1}}{d_{j_{m}}}-2 \quad \text { and } \quad 0 \leq k^{\prime} \leq \frac{\alpha d_{j_{m}}}{\operatorname{per} x_{l}} .
$$


Then

$$
\begin{aligned}
T^{n}\left(\sum_{s=1}^{j_{m}} z_{s}\right)-x_{l} & =T^{n_{j_{m}}+k^{\prime} \operatorname{per}\left(x_{l}\right)}\left(\sum_{s=1}^{j_{m}} z_{s}\right)-x_{l} \quad \text { by (i) } \\
& =T^{n_{j_{m}}+k^{\prime} \operatorname{per}\left(x_{l}\right)} z_{j_{m}}-T^{k^{\prime} \operatorname{per}\left(x_{l}\right)}\left(x_{l}-\sum_{s=1}^{j_{m}-1} z_{s}\right) \quad \text { by (iv). }
\end{aligned}
$$

Since $k^{\prime} \operatorname{per}\left(x_{l}\right) \leq \alpha d_{j_{m}}$, we deduce from (iii) that $\left\|T^{n}\left(\sum_{s=1}^{j_{m}} z_{s}\right)-x_{l}\right\| \leq 2^{-j_{m}}$.

- Suppose now that (18) has been proved up to the index $j-1$ for some $1 \leq j<j_{m+1}-j_{m}$, and that $n$ belongs to $A_{m, j}$. Write

$$
n=k d_{j_{m}+j}+i \quad \text { with } \quad i \in A_{m, j-1} \quad \text { and } \quad 0 \leq k \leq \frac{\alpha d_{j_{m}+j+1}}{d_{j_{m}+j}}-1 .
$$

Then

Thus

$$
\begin{aligned}
T^{n}\left(\sum_{s=1}^{j_{m}+j} z_{s}\right)-x_{l} & =T^{k d_{j_{m}+j}+i}\left(\sum_{s=1}^{j_{m}+j} z_{s}\right)-x_{l}=T^{i}\left(\sum_{s=1}^{j_{m}+j} z_{s}\right)-x_{l} \quad \text { by (i) } \\
& =T^{i}\left(\sum_{s=1}^{j_{m}+j-1} z_{s}\right)-x_{l}+T^{i} z_{j_{m}+j} .
\end{aligned}
$$

$$
\left\|T^{n}\left(\sum_{s=1}^{j_{m}+j} z_{s}\right)-x_{l}\right\|<\sum_{u=0}^{j-1} 2^{-\left(j_{m}+u\right)}+\left\|T^{i} z_{j_{m}+j}\right\|
$$

by the induction hypothesis. Now, $i \leq \alpha d_{j_{m}+j}$ by (16) since $i$ belongs to $A_{m, j-1}$, and thus $\left\|T^{i} z_{j_{m}+j}\right\|<2^{-\left(j_{m}+j\right)}$ by (i). Hence

$$
\left\|T^{n}\left(\sum_{s=1}^{j_{m}+j} z_{s}\right)-x_{l}\right\|<\sum_{u=0}^{j} 2^{-\left(j_{m}+u\right)},
$$

which concludes our induction. The inequality (18) being proved, we deduce that

$$
\left\|T^{n} z-x_{l}\right\|<\sum_{u \geq 0} 2^{-\left(j_{m}+u\right)}=2^{-\left(j_{m}-1\right)}
$$

for every $m \geq 1$ and every $n \in A_{m, j}, 0 \leq j<j_{m+1}-j_{m}$.

It follows from Fact 5.34 that given $\varepsilon>0$, there exists $m_{0} \geq 1$ such that $\mathcal{N}_{T}\left(z, B\left(x_{l}, \varepsilon\right)\right)$ contains the set

$$
\bigcup_{m \geq m_{0}}^{j_{m+1}-j_{m}-1} \bigcup_{j=0}^{A_{m, j}}
$$

So in order to show that $z$ is a frequently hypercyclic vector for $T$, it suffices to prove the following fact.

Fact 5.35. - The set $A:=\bigcup_{m \geq 1} \bigcup_{j=0}^{j_{m+1}-j_{m}-1} A_{m, j}$ has positive lower density.

Proof of Fact 5.35. - We start with a series of elementary remarks on the structure of the sets $A_{m, j}$. 
(a) $A_{m, 0} \subseteq\left[n_{j_{m}}, \alpha d_{j_{m}+1}\right]$ and $A_{m, j} \subseteq\left[d_{j_{m}+j}, \alpha d_{j_{m}+j+1}\right]$ for $1 \leq j<j_{m+1}-j_{m}$, so that the sets $A_{m, j}$ are contained in successive (disjoint) subintervals of $\mathbb{N}$.

(b) Inside each set $A_{m, j}, j \geq 1$, the translates $A_{m, j-1}+k d_{j_{m}+j}$ are pairwise disjoint.

(c) If we set $A_{m}:=\bigcup_{j=0}^{j_{m+1}-j_{m}-1} A_{m, j}$, then $A_{m} \subseteq\left[n_{j_{m}}, \alpha d_{j_{m+1}}\right]$. Since $\alpha d_{j_{m+1}}<n_{j_{m+1}}$, the sets $A_{m}, m \geq 1$, are thus contained in successive subintervals of $\mathbb{N}$;

(d) There is no redundancy in the definition of $A_{m, 0}$ : if

$$
n_{j_{m}}+k d_{j_{m}}+k^{\prime} \operatorname{per}\left(x_{l}\right)=n_{j_{m}}+\widetilde{k} d_{j_{m}}+\widetilde{k^{\prime}} \operatorname{per}\left(x_{l}\right)
$$

for some

$$
0 \leq k, k^{\prime} \leq \frac{\alpha d_{j_{m}+1}}{d_{j_{m}}}-2, \quad 0 \leq k^{\prime}, \widetilde{k^{\prime}} \leq \frac{\alpha d_{j_{m}}}{\operatorname{per}\left(x_{l}\right)},
$$

then $k=\widetilde{k}$ and $k^{\prime}=\widetilde{k^{\prime}}$. Indeed, we have $(k-\widetilde{k}) d_{j_{m}}=\left(k^{\prime}-\widetilde{k^{\prime}}\right) \operatorname{per}\left(x_{l}\right)$, so that $|k-\widetilde{k}| d_{j_{m}} \leq \alpha d_{j_{m}}$, and hence $k=\widetilde{k}$ because $\alpha<1$.

(e) It follows from (v) that

$$
\# A_{m, 0} \geq \frac{\alpha d_{j_{m}}}{\operatorname{per}\left(x_{l}\right)}\left(\frac{\alpha d_{j_{m}+1}}{d_{j_{m}}}-1\right) \geq \frac{\alpha d_{j_{m}}}{\operatorname{per}\left(x_{l}\right)} \cdot \frac{\alpha d_{j_{m}+1}}{2 d_{j_{m}}}=\frac{\alpha^{2} d_{j_{m}+1}}{2 \operatorname{per}\left(x_{l}\right)} .
$$

(f) By (a) and (v), we have for every $1 \leq j<j_{m+1}-j_{m}$ :

$$
\# A_{m, j} \geq\left(\frac{\alpha d_{j_{m}+j+1}}{d_{j_{m}+j}}-1\right) \# A_{m, j-1} \geq \frac{\alpha d_{j_{m}+j+1}}{2 d_{j_{m}+j}} \# A_{m, j-1}
$$

Iterating this inequality yields that

$$
\# A_{m, j} \geq\left(\frac{\alpha}{2}\right)^{j} \frac{d_{j_{m}+j+1}}{d_{j_{m}+1}} \# A_{m, 0} \geq \frac{\alpha^{j+2}}{2^{j+1}} \cdot \frac{d_{j_{m}+j+1}}{\operatorname{per}\left(x_{l}\right)} .
$$

Since $j_{m+1}-j_{m} \leq r_{l}$, this eventually gives

$$
\# A_{m, j} \geq \frac{\alpha^{r_{l}+2}}{2^{r_{l}+1}} \cdot \frac{d_{j_{m}+j+1}}{\operatorname{per}\left(x_{l}\right)} \text { for every } 1 \leq j<j_{m+1}-j_{m} .
$$

In order to prove that $A$ has positive lower density, we will show the existence of some positive number $\delta$ such that

$$
\frac{1}{n} \#([1, n] \cap A) \geq \delta \quad \text { for every } n \in A .
$$

This will be enough to ensure that $A$ has positive lower density. Indeed, if we enumerate the set $A$ as $A=\left\{a_{q} ; q \geq 1\right\}$ where $\left(a_{q}\right)_{q \geq 1}$ is strictly increasing, this inequality can be rewritten as $q / a_{q} \geq \delta$ for every $q \geq 1$, i.e. $a_{q} \leq q / \delta$; and this is easily seen to imply that $A$ has positive lower density.

We fix $m \geq 1$ and $n \in A_{m}$, and consider separately two cases.

- Suppose that $n \in A_{m, 0}$, and write

$$
n=n_{j_{m}}+k d_{j_{m}}+k^{\prime} \operatorname{per}\left(x_{l}\right), \quad \text { where } 0 \leq k \leq \frac{\alpha d_{j_{m}+1}}{d_{j_{m}}}-2 \text { and } 0 \leq k^{\prime} \leq \frac{\alpha d_{j_{m}}}{\operatorname{per}\left(x_{j}\right)} .
$$

Note that the set $A_{m-1}$ of (c) is contained in $[1, n]$ because $n \geq n_{j_{m}}>\alpha d_{j_{m}}$, and $A_{m-1}$ is disjoint from $A_{m, 0}$. So we have in particular

$$
\#([1, n] \cap A) \geq \# A_{m-1, j_{m}-j_{m-1}-1}+\#\left([1, n] \cap A_{m, 0}\right) .
$$

Moreover, the set $[1, n] \cap A_{m, 0}$ contains all integers of the form $n_{j_{m}}+s d_{j_{m}}+s^{\prime} \operatorname{per}\left(x_{l}\right)$, 
where $0 \leq s<k$ and $0 \leq s^{\prime} \leq \frac{\alpha d_{j m}}{\operatorname{per}\left(x_{l}\right)}$ (since all of them are in $A_{m, 0}$ and the largest is not greater than $\left.n_{j_{m}}+(k-1) d_{j_{m}}+\alpha d_{j_{m}} \leq n_{j_{m}}+k d_{j_{m}} \leq n\right)$. Hence,

$$
\#\left([1, n] \cap A_{m, 0}\right) \geq k \cdot \frac{\alpha d_{j_{m}}}{\operatorname{per}\left(x_{l}\right)} .
$$

By (f), it follows that

$$
\#([1, n] \cap A) \geq \frac{\alpha^{r_{l}+2} d_{j_{m}}}{2^{r_{l}+1} \operatorname{per}\left(x_{l}\right)}+k \frac{\alpha d_{j_{m}}}{\operatorname{per}\left(x_{l}\right)} \geq \frac{\alpha^{r_{l}+2}}{2^{r_{l}+1}} \cdot \frac{(k+1) d_{j_{m}}}{\operatorname{per}\left(x_{l}\right)} .
$$

Since $n \leq n_{j_{m}}+(k+\alpha) d_{j_{m}} \leq 2(k+1) d_{j_{m}}$, we conclude that

$$
\#([1, n] \cap A) \geq \frac{\alpha^{r_{l}+2}}{2^{r_{l}+2} \operatorname{per}\left(x_{l}\right)} n .
$$

- Suppose now that $n \in A_{m, j}$ for some $1 \leq j<j_{m+1}-j_{m}$, so that

$$
n=k d_{j_{m}+j}+i, \quad \text { with } 1 \leq k \leq \frac{\alpha d_{j_{m}+j+1}}{d_{j_{m}+j}}-1 \text { and } i \in A_{m, j-1} .
$$

Then $\max A_{m, j-1} \leq n \leq(k+1) d_{j_{m}+j}$ by (16). In particular,

$$
\#([1, n] \cap A) \geq \# A_{m, j-1}+\#\left([1, n] \cap A_{m, j}\right) \text {. }
$$

Moreover, since $n \geq k d_{j_{m}+j} \geq(k-1) d_{j_{m}+j}+\max A_{m, j-1}$ and the translates $A_{m, j-1}+$ $l d_{j_{m}+j}, l<k$ are pairwise disjoint (and contained in $A_{m, j}$ ), we also have

$$
\text { \# }\left([1, n] \cap A_{m, j}\right) \geq(k-1) \# A_{m, j-1} .
$$

By (f), it follows that

$$
\#([1, n] \cap A) \geq k \frac{\alpha^{r_{l}+2} d_{j_{m}+j}}{2^{r_{l}+1} \operatorname{per}\left(x_{l}\right)} \geq \frac{1}{2} \cdot \frac{\alpha^{r_{l}+2}}{2^{r_{l}+1}} \cdot \frac{1}{\operatorname{per}\left(x_{l}\right)} \cdot n,
$$

where we have used the inequality $n \leq(k+1) d_{j_{m}+j}$.

We have thus proved that

$$
\#([1, n] \cap A) \geq \frac{\alpha^{r_{l}+2}}{2^{r_{l}+2} \operatorname{per}\left(x_{l}\right)} n \quad \text { for every } n \in A,
$$

which concludes the proof of Fact 5.35,

The proof of Theorem 5.31 is now complete.

5.4.1. Link with the Operator Specification Property. - We conclude this section by a result which shows that many operators which are known to be frequently hypercyclic satisfy the assumption of Theorem 5.31. This is the case for all operators which satisfy the Frequent Hypercyclicity Criterion and, more generally, for operators with the so-called Operator Specification Property.

This last property, which has been recently introduced and studied by Bartoll, MartínezGiménez, and Peris (10, 2]), is the linear version of the classical Specification Property for compact dynamical systems introduced by Bowen in $\mathbf{1 5}$. The definition reads as follows: an operator $T \in \mathfrak{B}(X)$ has the Operator Specification Property (OSP) if there exists an increasing sequence $\left(K_{m}\right)_{m \geq 1}$ of $T$-invariant subsets of $X$ with $0 \in K_{1}$, the union of which is dense in $X$, such that for each $m \geq 1$, the restriction of $T$ to $K_{m}$ has the Specification Property in the sense of [15], which means that the following holds true: 
(*) for every $\delta>0$, there exists an integer $N_{\delta, m} \geq 1$ such that for every finite family $y_{1}, \ldots, y_{s}$ of points of $K_{m}$, and any integers $0=j_{1} \leq k_{1}<j_{2} \leq k_{2}<\cdots<j_{s} \leq k_{s}$ with $j_{r+1}-k_{r} \geq N_{\delta, m}$ for every $1 \leq r \leq s-1$, there is a point $x \in K_{m}$ such that for every $1 \leq r \leq s$

$$
\sup _{j_{r} \leq i \leq k_{r}}\left\|T^{i} x-T^{i} y_{r}\right\|<\delta \quad \text { while } \quad T^{N_{\delta, m}+k_{s}} x=x .
$$

Stated in an informal way, $(*)$ means that arbitrary large pieces of the orbits of finitely many points of $K_{m}$ can be approximated by the orbits of a single periodic point of $K_{m}$, provided that the gaps between the different sets of indices where we require this approximation are sufficiently large.

It is proved in 2 that operators with the OSP are chaotic, topologically mixing, and frequently hypercyclic. Moreover, any operator satisfying the general version of the Frequent Hypercyclicity Criterion given in [13 has the OSP. We prove in Theorem 5.36 below that operators with the OSP satisfy the assumption of Theorem 5.31. This was pointed out to us by Alfred Peris, who kindly allowed us to reproduce his proof here. This improves on a previous observation (proved in a preliminary version of this paper) according to which operators satisfying the Frequent Hypercyclicity Criterion also satisfy the assumptions of Theorem 5.31.

Theorem 5.36. - Let $T \in \mathfrak{B}(X)$ be an operator with the OSP. There exists a dense subspace $X_{0}$ of $X$ with $T\left(X_{0}\right) \subseteq X_{0}$ and $X_{0} \subseteq \operatorname{Per}(T)$, such that, for every $\alpha \in(0,1 / 2)$, every $x \in X_{0}$, every $\varepsilon>0$, and every integer $d_{0} \geq 1$, there exist $z \in X_{0}$ and integers $n$, $d \geq 1$ such that properties (0), (1), and (2) of Theorem 5.31 hold true.

Proof. - Let $\left(K_{m}\right)_{m \geq 1}$ be an increasing sequence of $T$-invariant subsets of $X$ with $0 \in K_{1}$, the union of which is dense in $X$, such that $(*)$ above holds true for every $m \geq 1$. We define $X_{0}:=\operatorname{span}\left[\bigcup_{m \geq 1} K_{m} \cap \operatorname{Per}(T)\right]$, which is clearly $T$-invariant and dense in $X$.

Let us fix $x \in X_{0}, \varepsilon>0$ and $d_{0} \geq 1$. The vector $x$ can be written as $x=\sum_{m=1}^{m_{0}} a_{m} x_{m}$, where $a_{m}$ is a scalar and $x_{m}$ is a vector belonging to $K_{m}$ for every $1 \leq m \leq m_{0}$. By [2. Prop. 10], the map induced by $T$ on the set $K=\sum_{m=1}^{m_{0}} a_{m} K_{m}$ has the Specification Property. So we can assume without loss of generality that $x$ belongs to $K_{m}$ for some $m \geq 1$. Let now $N_{\varepsilon, m}$ be such that property $(*)$ above holds true, and let $d \geq 1$ be a multiple of $d_{0}$ so large that $\alpha d+N_{\varepsilon, m}<d / 2$. We then define $d^{\prime}$ to be the integer part of $\alpha d, n=N_{\varepsilon, m}+d^{\prime}, y_{1}=0$, and $y_{2}=T^{l-n} x$, where $l$ is any multiple of per $(x)$ such that $l>n$. We also set $j_{1}=0, k_{1}=d^{\prime}, j_{2}=n$, and $k_{2}=d-N_{\varepsilon, m}$. As $j_{2}-k_{1}=n-d^{\prime}=N_{\varepsilon, m}$, the Specification Property on $K_{m}$ implies that there exists a point $z \in K_{m}$ such that

$$
\begin{aligned}
\left\|T^{k} z-T^{k} y_{1}\right\| & =\left\|T^{k} z\right\|<\varepsilon \quad \text { for every } 0 \leq k \leq d^{\prime} ; \\
\left\|T^{k} z-T^{k} y_{2}\right\| & <\varepsilon \quad \text { for every } n \leq k \leq k_{2} ; \\
T^{N_{\varepsilon, m}+k_{2}} z & =T^{d} z=z .
\end{aligned}
$$

In other words,

$$
\begin{aligned}
\left\|T^{k} z\right\|<\varepsilon & \text { for every } 0 \leq k \leq \alpha d ; \\
\left\|T^{n+k} z-T^{k} x\right\|<\varepsilon & \text { for every } 0 \leq k \leq k_{2}-n
\end{aligned}
$$

since $T^{n+k} y_{2}=T^{n+k+l-n} x=T^{k} x$; and $d$ is a multiple of the period of $z$. Since $k_{2}-n=$ $d-2 N_{\varepsilon, m}-d^{\prime} \geq \alpha d$, this shows that assumptions (0), (1), and (2) of Theorem 5.31 are satisfied. 
Remark 5.37. - The converse of Theorem 5.36] is not true; that is, operators satisfying the assumptions of Theorem 5.31 need not have the OSP. Indeed, we will construct in Section 6 (more precisely, in Example 6.23) some operators which satisfy the assumptions of Theorem 5.31 and yet are not topologically mixing.

To conclude this section, we essentially show that the OSP implies ergodicity. This is coherent with what happens in the non-linear setting: it is proved in $\mathbf{5 0}$ that compact dynamical systems with the Specification Property are ergodic. They actually enjoy a much stronger property: given such a system $(X, T)$, the ergodic measures with full support for $(X, T)$ are comeager in the set $\mathcal{P}_{T}(X)$ of $T$-invariant probability measures endowed with its natural Polish topology. On the other hand, we do not know whether operators with the OSP are mixing (i.e. admit mixing measures with full support), whereas it is known that the Frequent Hypercyclicity Criterion does imply mixing, and in fact mixing in the Gaussian sense (see [41] and [8]).

Proposition 5.38. - If $T \in \mathfrak{B}(X)$ satisfies the OSP, and if the sequence $\left(K_{m}\right)_{m \geq 1}$ of $T$-invariant subsets of $X$ appearing in the definition of the OSP consists of compact sets, then $T$ is ergodic.

The assumption of Proposition 5.38 that the sets $K_{m}, m \geq 1$, be compact seems to be no real restriction. As mentioned in [2], all known examples of operators with the OSP do satisfy the OSP with respect to a sequence of $T$-invariant compact subsets $\left(K_{m}\right)_{m \geq 1}$ of $X$.

Proof. - Let us denote by $\mathcal{P}(X)$ the space of all Borel probability measures on $X$ endowed with its natural Polish topology (a sequence $\left(\mu_{n}\right)_{n \geq 1}$ of elements of $\mathcal{P}(X)$ converges to $\mu \in \mathcal{P}(X)$ if and only if $\int_{X} f d \mu_{n}$ tends to $\int_{X} f d \mu$ as $n$ tends to infinity for every bounded continuous function $f$ from $X$ into $\mathbb{R})$, and by $\mathcal{P}_{T}(X) \subseteq \mathcal{P}(X)$ the set of all $T$-invariant measures. Then $\mathcal{P}_{T}(X)$ is a Polish space, being a closed subset of $\mathcal{P}(X)$. We denote by $\mathcal{E}_{T}(X) \subseteq \mathcal{P}_{T}(X)$ the set of all ergodic measures for $T$. For any family of measures $\mathcal{M} \subseteq$ $\mathcal{P}(X)$, we denote by $\mathcal{M}^{*}$ the family of all measures $\mu \in \mathcal{M}$ with full support. Finally, for any subset $\mathcal{M}$ of $\mathcal{P}(X)$ and any Borel subset $A$ of $X$, we set $\mathcal{M}(A):=\{\mu \in \mathcal{M} ; \mu(A)=1\}$.

Let $\left(K_{m}\right)_{m \geq 1}$ be an increasing sequence of compact $T$-invariant subsets of $X$ with $\overline{\bigcup_{m>1} K_{m}}=X$ such that $T_{\mid K_{m}}$ has the Specification Property for every $m \geq 1$. By a result of [50], we know that for each $m \geq 1$, the set $\mathcal{E}_{T}^{*}\left(K_{m}\right)$ of all measures $\mu \in \mathcal{E}_{T}(X)$ with support equal to $K_{m}$ is dense in $\mathcal{P}_{T}\left(K_{m}\right)$. Let us now denote by $\mathcal{M}$ the closure of $\bigcup_{m \geq 1} \mathcal{P}_{T}\left(K_{m}\right)$ in $\mathcal{P}_{T}(X)$.

Fact 5.39. - The set $\mathcal{E}_{T}(X) \cap \mathcal{M}$ is a dense $G_{\delta}$ subset of $\mathcal{M}$.

Proof of Fact [5.39] - It is known that $\mathcal{E}_{T}(X)$ is a $G_{\delta}$ subset of $\mathcal{P}_{T}(X)$ (see 42 for a detailed proof), so that $\mathcal{E}_{T}(X) \cap \mathcal{M}$ is $G_{\delta}$ in $\mathcal{M}$. Moreover, since $\mathcal{E}_{T}\left(K_{m}\right)$ is dense in $\mathcal{P}_{T}\left(K_{m}\right)$ for each $m$ by [50], it is clear that $\mathcal{E}_{T}(X) \cap \mathcal{M}$ is dense in $\mathcal{M}$.

Fact 5.40. - The set $\mathcal{M}^{*}$ is a dense $G_{\delta}$ subset of $\mathcal{M}$.

Proof of Fact 5.40 - Let $\left(O_{p}\right)_{p \geq 1}$ be a countable basis of non-empty open subsets of $X$. Set, for each $p \geq 1, \mathcal{O}_{p}:=\left\{\mu \in \mathcal{P}(X) ; \mu\left(O_{p}\right)>0\right\}$. Then each set $\mathcal{O}_{p}$ is open in $\mathcal{P}(X)$, and moreover

$$
\mathcal{M}^{*}=\mathcal{M} \cap\left(\bigcap_{p \geq 1} \mathcal{O}_{p}\right)
$$


So we just have to show that each set $\mathcal{M} \cap \mathcal{O}_{p}$ is dense in $\mathcal{M}$. Let us fix $p \geq 1$, and let $\mathcal{U}$ be a non-empty open subset of $\mathcal{M}$. Since the sequence $\left(K_{m}\right)_{m>1}$ is increasing, the definition of $\mathcal{M}$ implies that $\mathcal{U} \cap \mathcal{P}_{T}\left(K_{m}\right)$ is non-empty for all $m$ sufficiently large. Moreover, since $\bigcup_{m>1} K_{m}$ is dense in $X$ and $\left(K_{m}\right)_{m \geq 1}$ is increasing, $K_{m} \cap O_{p}$ is non-empty too for all $m$ sufficiently large. So there exists $m \geq 1$ such that $K_{m} \cap O_{p}$ and $\mathcal{U} \cap \mathcal{P}_{T}\left(K_{m}\right)$ are both non-empty. Since $\mathcal{E}_{T}^{*}\left(K_{m}\right)$ is dense in $\mathcal{P}_{T}\left(K_{m}\right)$ and $\mathcal{U} \cap \mathcal{P}_{T}\left(K_{m}\right)$ is open in $\mathcal{P}_{T}\left(K_{m}\right)$, it follows that there exists a measure $\mu \in \mathcal{E}_{T}(X)$ with support equal to $K_{m}$ such that $\mu$ belongs to $\mathcal{U}$ and $\mu\left(O_{p}\right)>0$. Hence, we have shown that $\mathcal{U} \cap \mathcal{O}_{p}$ is non-empty.

The two facts above combined with the Baire Category Theorem applied in $\mathcal{M}$ imply that $\mathcal{E}_{T}(X) \cap \mathcal{M}^{*}$ is non-empty. In particular $\mathcal{E}_{T}(X)^{*}$ is non-empty, i.e. $T$ is ergodic.

Remark 5.41. - We will see in Section [ that the assumptions of Theorem 5.31 (which are sufficient for frequent hypercyclicity) do not imply ergodicity. So Proposition 5.38 makes the difference between the OSP and our criterion for frequent hypercyclicity all the more tangible.

\section{Special examples of hypercyclic operators}

In this section, we introduce some particular classes of operators, which are defined on any space $\ell_{p}(\mathbb{N}), 1 \leq p<\infty$. We do not restrict ourselves to the Hilbertian case $p=2$, because the general case adds no extra complication. It is within these classes that we will exhibit operators which are chaotic and frequently hypercyclic but not ergodic, operators which are chaotic and $\mathcal{U}$-frequently hypercyclic but not frequently hypercyclic and operators which are chaotic and topologically mixing but not $\mathcal{U}$-frequently hypercyclic.

A word of caution: for technical reasons, we have decided that $\mathbb{N}$ starts at 0 ; that is, $\mathbb{N}=\{0,1,2, \ldots\}$. Accordingly, the canonical basis of $\ell_{p}(\mathbb{N})$ will be denoted by $\left(e_{k}\right)_{k \geq 0}$. Also, we denote by $c_{00}$ the linear span of the vectors $e_{k}, k \geq 0$, i.e. the subspace of $\ell_{p}(\mathbb{N})$ consisting of all finitely supported vectors.

6.1. Operators of C-type: basic facts. - In view of our criteria for $\mathcal{U}$-frequent hypercyclicity and frequent hypercyclicity relying on the existence of periodic points, we would like to find a rich family of operators for which we can easily find a large supply of periodic points. For example, we could consider operators $T_{w, b}$ defined by

$$
T_{w, b} e_{k}=\left\{\begin{array}{cl}
w_{k+1} e_{k+1} & \text { if } k \in\left[b_{n}, b_{n+1}-1\right) \\
\left(\prod_{j=b_{n}+1}^{b_{n+1}-1} w_{j}\right)^{-1} e_{b_{n}} & \text { if } k=b_{n+1}-1 \text { with } n \geq 0
\end{array}\right.
$$

where $w=\left(w_{j}\right)_{j \geq 1}$ is a weight sequence and $b=\left(b_{n}\right)_{n \geq 1}$ is a strictly increasing sequence of integers with $b_{0}=0$. Indeed, with this definition it is clear that (whatever the choice of $w$ and $b$ ) every basis vector $e_{k}$ and hence every vector $x \in c_{00}$ is periodic for $T_{w, b}$.

However, none of these operators is hypercyclic since they are direct sums of finitedimensional operators, and there exist no hypercyclic operators in finite dimension. It is thus necessary to perturb the operators $T_{w, b}$ in order to obtain an interesting family of hypercyclic operators in which finite sequences are still periodic. For reasons that will be explained below, the operators in this family will be called operators of $C$-type. Any operator of C-type will be associated to four parameters $v, w, \varphi$, and $b$, where

- $v=\left(v_{n}\right)_{n \geq 1}$ is a sequence of non-zero complex numbers such that $\sum_{n \geq 1}\left|v_{n}\right|<\infty$; 
- $w=\left(w_{j}\right)_{j \geq 1}$ is a sequence of complex numbers which is both bounded and bounded below, i.e. $0<\inf _{k \geq 1}\left|w_{k}\right| \leq \sup _{k \geq 1}\left|w_{k}\right|<\infty$;

- $\varphi$ is a map from $\mathbb{N}$ into itself, such that $\varphi(0)=0, \varphi(n)<n$ for every $n \geq 1$, and the set $\varphi^{-1}(l)=\{n \geq 0 ; \varphi(n)=l\}$ is infinite for every $l \geq 0$;

- $b=\left(b_{n}\right)_{n \geq 0}$ is a strictly increasing sequence of positive integers such that $b_{0}=0$ and $b_{n+1}-b_{n}$ is a multiple of $2\left(b_{\varphi(n)+1}-b_{\varphi(n)}\right)$ for every $n \geq 1$.

Definition 6.1. - The operator of $C$-type $T_{v, w, \varphi, b}$ on $\ell_{p}(\mathbb{N})$ associated to the data $v$, $w, \varphi$, and $b$ given as above is defined by

$$
T_{v, w, \varphi, b} \quad e_{k}= \begin{cases}w_{k+1} e_{k+1} & \text { if } k \in\left[b_{n}, b_{n+1}-1\right), n \geq 0, \\ v_{n} e_{b_{\varphi(n)}}-\left(\prod_{j=b_{n}+1}^{b_{n+1}-1} w_{j}\right)^{-1} e_{b_{n}} & \text { if } k=b_{n+1}-1, n \geq 1 \\ -\left(\prod_{j=b_{0}+1}^{b_{1}-1} w_{j}\right)^{-1} e_{0} & \text { if } k=b_{1}-1 .\end{cases}
$$

Note that by convention, an empty product is declared to be be equal to 1 ; that is, $\prod_{j=b_{n}+1}^{b_{n+1}-1} w_{j}=1$ when $b_{n+1}=b_{n}+1$ (which can happen only for $\left.n=0\right)$.

Without any additional assumption on the parameters, these formulas define a linear map on $c_{00}$ only. The first issue is of course the boundedness of $T_{v, w, \varphi, b}$.

Fact 6.2. - The operator $T_{v, w, \varphi, b}$ is well-defined and bounded on $\ell_{p}(\mathbb{N})$ as soon as that the following condition holds true:

$$
\inf _{n \geq 0} \prod_{b_{n}<j<b_{n+1}}\left|w_{j}\right|>0 .
$$

Proof. - This is rather clear. Indeed, it appears that $T_{v, w, \varphi, b}$ can be written as

$$
T_{v, w, \varphi, b}=\bigoplus_{n \geq 0} C_{w, b, n}+R_{v, b},
$$

where $R_{v, b}$ is the operator defined by

$$
R_{v, b} x=\sum_{n \geq 1} v_{n} x_{b_{n+1}-1} e_{b_{\varphi(n)}}, \quad x \in \ell_{p}(\mathbb{N})
$$

which is clearly bounded (and even compact) because $\sum_{n \geq 1}\left|v_{n}\right|<\infty$, and, for each $n \geq 1$, $C_{w, b, n}$ is a finite-dimensional cyclic operator acting on $E_{n}:=\operatorname{span}\left[e_{k} ; b_{n} \leq k \leq b_{n+1}-1\right]$. Condition (20) implies that $\sup _{n \geq 1}\left\|C_{w, b, n}\right\|$ is finite, and $T_{v, w, \varphi, b}$ is thus bounded.

From now on, we will always assume that Condition (20) is satisfied. Also, when no confusion arises, we will write simply $T$ instead of $T_{v, w, \varphi, b}$.

Remark 6.3. - We call such operators operators of C-type for two different reasons. On the one hand, "C" stands for "cyclic": as we have just explained, each operator $T_{v, w, \varphi, b}$ is a compact perturbation of an infinite direct sum of cyclic finite-dimensional operators $\bigoplus_{n>0} C_{w, b, n}$, where $C_{w, b, n}$ is defined on $E_{n}=\operatorname{span}\left[e_{k} ; b_{n} \leq k \leq b_{n+1}-1\right]$. On the other hand, we will see in a moment that, as a consequence of their particular structure, the operators $T_{v, w, \varphi, b}$ happen to be chaotic under a very mild restriction on the parameters; so, "C" stands for "chaotic" as well. 
The following identity will be used repeatedly: if $T=T_{v, w, \varphi, b}$ is an operator of C-type on $\ell_{p}(\mathbb{N})$, then

$$
T^{b_{n+1}-b_{n}} e_{b_{n}}=v_{n}\left(\prod_{j=b_{n}+1}^{b_{n+1}-1} w_{j}\right) e_{b_{\varphi(n)}}-e_{b_{n}} \quad \text { for every } n \geq 1 .
$$

This allows to show that operators of C-type always have plenty of periodic points:

Fact 6.4. - If $T=T_{v, w, \varphi, b}$ is an operator of C-type on $\ell_{p}(\mathbb{N})$, then every basis vector $e_{k}$ is periodic for $T_{v, w, \varphi, b}$; more precisely,

$$
T^{2\left(b_{n+1}-b_{n}\right)} e_{k}=e_{k} \quad \text { if } k \in\left[b_{n}, b_{n+1}\right), n \geq 0 .
$$

Consequently, every vector $x \in c_{00}$ is periodic for $T_{v, w, \varphi, b}$, and hence $T_{v, w, \varphi, b}$ has a dense set of periodic points.

Proof. - Since $e_{k}$ is a non-zero multiple of $T^{k-b_{n}} e_{b_{n}}$ for every $b_{n} \leq k<b_{n+1}$, it suffices to prove that $T^{2\left(b_{n+1}-b_{n}\right)} e_{b_{n}}=e_{b_{n}}$ for every $n \geq 0$. We prove this by induction on $n$.

- If $n=0$, then $T^{b_{1}-b_{0}} e_{0}=-e_{0}$ by definition of $T e_{b_{1}-1}$, and thus $T^{2\left(b_{1}-b_{0}\right)} e_{0}=e_{0}$.

- Let $n \geq 1$, and assume that the result has been proved for all $m<n$. We know that $b_{n+1}-b_{n}$ is a multiple of $2\left(b_{\varphi(n)+1}-b_{\varphi(n)}\right)$ and since $\varphi(n)<n$, it follows by (21) and the induction hypothesis that

$$
T^{2\left(b_{n+1}-b_{n}\right)} e_{b_{n}}=v_{n}\left(\prod_{j=b_{n}+1}^{b_{n+1}-1} w_{j}\right) e_{b_{\varphi(n)}}-T^{b_{n+1}-b_{n}} e_{b_{n}}=e_{b_{n}} .
$$

Using the above fact and Corollary 5.13, we can now obtain the following sufficient condition for an operator of C-type to be chaotic.

Proposition 6.5. - Suppose that

$$
\limsup _{\substack{N \rightarrow \infty \\ N \in \varphi^{-1}(n)}}\left|v_{N}\right| \prod_{j=b_{N}+1}^{b_{N+1}-1}\left|w_{j}\right|=\infty \quad \text { for every } n \geq 0 .
$$

Then the operator of $C$-type $T=T_{v, w, \varphi, b}$ on $\ell_{p}(\mathbb{N})$ is chaotic.

Proof. - We apply Corollary [5.13 with $X_{0}:=\left\{e_{k} ; k \geq 0\right\}$. Fix $k \geq 0$ and $\varepsilon>0$. We are looking for a vector $z \in \operatorname{Per}(T)$ and an integer $m \geq 1$ such that $\|z\|<\varepsilon$ and $\left\|T^{m} z-e_{k}\right\|<\varepsilon$. Let $n \geq 0$ be such that $k$ belongs to $\left[b_{n}, b_{n+1}\right)$. By the assumption of Proposition 6.5, there exists $N \in \varphi^{-1}(n)$ such that

$$
\left|v_{N}\right| \prod_{j=b_{N}+1}^{b_{N+1}-1}\left|w_{j}\right|>\frac{1}{\varepsilon}\left(\prod_{j=b_{n}+1}^{k}\left|w_{j}\right|\right)^{-1} \max \left\{1,\|w\|_{\infty}\right\}^{k-b_{n}} .
$$

The vector

$$
z:=v_{N}^{-1}\left(\prod_{j=b_{N}+1}^{b_{N+1}-1} w_{j}\right)^{-1}\left(\prod_{j=b_{n}+1}^{k} w_{j}\right)^{-1} e_{b_{N}}
$$


is periodic by Fact 6.4, and satisfies $\|z\|<\varepsilon$ by (22). Moreover, since $\varphi(N)=n$, we have by (21):

$$
\begin{aligned}
T^{b_{N+1}-b_{N}+k-b_{n}} e_{b_{N}} & =v_{N}\left(\prod_{j=b_{N}+1}^{b_{N+1}-1} w_{j}\right) T^{k-b_{n}} e_{b_{n}}-T^{k-b_{n}} e_{b_{N}} \\
& =v_{N}\left(\prod_{j=b_{N}+1}^{b_{N+1}-1} w_{j}\right)\left(\prod_{j=b_{n}+1}^{k} w_{j}\right) e_{k}-\left(\prod_{j=b_{N}+1}^{b_{N}+k-b_{n}} w_{j}\right) e_{b_{N}+k-b_{n}} .
\end{aligned}
$$

By definition of $z$, this implies that

$$
T^{b_{N+1}-b_{N}+k-b_{n}} z=e_{k}-\left(\prod_{j=b_{N}+1}^{b_{N}+k-b_{n}} w_{j}\right) v_{N}^{-1}\left(\prod_{j=b_{N}+1}^{b_{N+1}-1} w_{j}\right)^{-1}\left(\prod_{j=b_{N}+1}^{k} w_{j}\right)^{-1} e_{b_{N}+k-b_{n}} ;
$$

and by (22), it follows that $\left\|T^{b_{N+1}-b_{N}+k-b_{n}} z-e_{k}\right\|<\varepsilon$. The assumptions of Corollary 5.13 are thus satisfied, and $T$ is chaotic.

Recall that every chaotic operator is topologically weakly mixing [7, Ch. 4] and reiteratively hypercyclic [12. In the following subsections, we will be interested in frequent hypercyclicity, $\mathcal{U}$-frequent hypercyclicity and topological mixing for operators of C-type.

6.2. Operators of $\mathbf{C}_{+}$-type: how to be FHC or UFHC. - For the construction of our counterexamples, we will work with operators of C-type for which the data $v, w, \varphi$ and $b$ have a special structure. For every integer $k \geq 1$, we require that:

- $\varphi(n)=n-2^{k-1}$ for every $n \in\left[2^{k-1}, 2^{k}\right)$, so that $\varphi\left(\left[2^{k-1}, 2^{k}\right)\right)=\left[0,2^{k-1}\right)$;

- the blocks $\left[b_{n}, b_{n+1}\right), n \in\left[2^{k-1}, 2^{k}\right)$, all have the same size, which we denote by $\Delta^{(k)}$ :

$$
b_{n+1}-b_{n}=\Delta^{(k)} \quad \text { for every } n \in\left[2^{k-1}, 2^{k}\right) ;
$$

- the sequence $v$ is constant on the interval $\left[2^{k-1}, 2^{k}\right)$ : there exists $v^{(k)}$ such that

$$
v_{n}=v^{(k)} \quad \text { for every } n \in\left[2^{k-1}, 2^{k}\right) \text {; }
$$

- the sequences of weights $\left(w_{b_{n}+i}\right)_{1 \leq i<\Delta^{(k)}}$ are independent of $n \in\left[2^{k-1}, 2^{k}\right)$ : there exists a sequence $\left(w_{i}^{(k)}\right)_{1 \leq i<\Delta^{(k)}}$ such that

$$
w_{b_{n}+i}=w_{i}^{(k)} \quad \text { for every } 1 \leq i<\Delta^{(k)} \text { and every } n \in\left[2^{k-1}, 2^{k}\right) .
$$

If these conditions are met, we say that $T_{v, w, \varphi, b}$ is an operator of $C_{+}$-type.

Remark 6.6. - By definition, the map $\varphi$ is the same for all operators of $\mathrm{C}_{+}$-type, so it is no longer a "parameter". However, we will continue using the notation $T_{v, w, \varphi, b}$.

Our first result concerning these operators gives a sufficient set of conditions for a $\mathrm{C}_{+}-$ type operator to be $\mathcal{U}$-frequently hypercyclic. This will be deduced from Theorem 5.14 .

Theorem 6.7. - Let $T=T_{v, w, \varphi, b}$ be an operator of $C_{+}$-type on $\ell_{p}(\mathbb{N})$. Suppose that there exists a constant $\alpha>0$ such that the following property holds true : for every $C \geq 1$ and every integer $k_{0} \geq 1$, there exist two integers $k>k_{0}$ and $1 \leq m<\Delta^{(k)}$ such that

$$
\left|v^{(k)}\right| \prod_{i=\Delta^{(k)}-m}^{\Delta^{(k)}-1}\left|w_{i}^{(k)}\right| \geq C \quad \text { and } \quad\left|v^{(k)}\right| \prod_{i=m^{\prime}+1}^{\Delta^{(k)}-1}\left|w_{i}^{(k)}\right|>C \quad \text { for every } 0 \leq m^{\prime} \leq \alpha m .
$$

Then $T$ is chaotic and $\mathcal{U}$-frequently hypercyclic. 
Proof. - The following fact will be useful.

Fact 6.8. - Let $k \geq 1$. For any $l<2^{k-1}$ and $1 \leq s \leq \Delta^{(k)}$, we have

$$
T^{s} e_{2_{2^{k-1}+l+1}-s}=v^{(k)}\left(\prod_{i=\Delta^{(k)}-s+1}^{\Delta^{(k)}-1} w_{i}^{(k)}\right) e_{b_{l}}-\left(\prod_{i=1}^{\Delta^{(k)}-s} w_{i}^{(k)}\right)^{-1} e_{b_{2^{k-1}+l}} .
$$

Proof of Fact 6.8. - Since $l<2^{k-1}$, we have $\varphi\left(2^{k-1}+l\right)=l$. So the formula follows directly from the definition of $T$.

We are going to show that the assumption of Theorem 5.14 is satisfied with $X_{0}=$ $\operatorname{span}\left[e_{k} ; k \geq 0\right]$. So let us fix $x \in X_{0}$ and $\varepsilon>0$. We choose $k_{0} \geq 1$ such that $x$ may be written as

$$
x=\sum_{l<2^{k_{0}}} \sum_{j=b_{l}}^{b_{l+1}-1} x_{j} e_{j} .
$$

Let $C>0$ be a very large number, to be specified later on in the proof. By assumption, there exist $k>k_{0}$ and $1 \leq m<\Delta^{(k)}$ such that

$$
\left|v^{(k)}\right| \prod_{i=\Delta^{(k)}-m}^{\Delta^{(k)}-1}\left|w_{i}^{(k)}\right|>C
$$

and

$$
\left|v^{(k)}\right| \prod_{i=m^{\prime}+1}^{\Delta^{(k)}-1}\left|w_{i}^{(k)}\right|>C \quad \text { for every } 0 \leq m^{\prime} \leq \alpha m .
$$

Note that since the sequences $v$ and $w$ are bounded, it follows from (23) that the integer $m$ can be chosen as large as we please, provided that $C$ is large enough. So we may assume from the beginning that $m>2 \Delta^{\left(k_{0}\right)}$. We will also assume that $\alpha<1$, which will be useful below.

We set

$$
z:=\sum_{l<2^{k_{0}}} \sum_{j=b_{l}}^{b_{l+1}-1} x_{j}\left(v^{(k)} \prod_{i=\Delta^{(k)}-m+j-b_{l}+1}^{\Delta^{(k)}-1} w_{i}^{(k)}\right)^{-1}\left(\prod_{i=1}^{j-b_{l}} w_{b_{l}+i}\right)^{-1} e_{b_{2^{k-1}+l+1}-m+j-b_{l}} .
$$

Our aim is to prove that if $C$ has been suitably chosen, then

$$
\|z\|<\varepsilon \quad \text { and } \quad\left\|T^{m+m^{\prime}} z-T^{m^{\prime}} x\right\|<\varepsilon \quad \text { for every } 0 \leq m^{\prime} \leq \alpha m / 2 .
$$

Theorem 5.14 will then conclude the proof.

The first of these two claims is the easiest one to prove. Indeed, since the weight sequence $w$ is bounded and bounded from below, it follows from (23) that

$$
\|z\| \leq\|x\| \cdot C^{-1} \cdot A^{\Delta^{\left(k_{0}\right)}},
$$

where $A$ is an absolute constant. So $\|z\|<\varepsilon$ if $C$ is large enough.

Let us now estimate the norm of the vector $T^{m+m^{\prime}} z-T^{m^{\prime}} x$ for every $0 \leq m^{\prime} \leq \alpha m / 2$. Note that if $0 \leq l \leq 2^{k_{0}}-1$ and $b_{l} \leq j<b_{l+1}$, then $m-\left(j-b_{l}\right) \geq 1$ since $0 \leq j-b_{l}<\Delta^{\left(k_{0}\right)}$ 
and $m>\Delta^{\left(k_{0}\right)}$. Applying Fact 6.8 with $s:=m-\left(j-b_{l}\right)$, we get

$$
\begin{aligned}
T^{m-\left(j-b_{l}\right)} e_{b_{2^{k-1}+l+1}-m+j-b_{l}}=v^{(k)} & \left(\prod_{i=\Delta^{(k)}-m+j-b_{l}+1}^{\Delta^{(k)}-1} w_{i}^{(k)}\right) e_{b_{l}} \\
& -\left(\prod_{i=1}^{\Delta^{(k)}-m+j-b_{l}} w_{i}^{(k)}\right)^{-1} e_{b_{2^{k-1}+l}},
\end{aligned}
$$

and hence

$$
\begin{aligned}
T^{m} e_{b_{2^{k-1}+l+1}-m+j-b_{l}}= & \left(v^{(k)} \prod_{i=\Delta^{(k)}-m+j-b_{l}+1}^{\Delta^{(k)}-1} w_{i}^{(k)}\right)\left(\prod_{i=1}^{j-b_{l}} w_{b_{l}+i}\right) e_{j} \\
& -\left(\prod_{i=j-b_{l}+1}^{\Delta^{(k)}-m+j-b_{l}} w_{i}^{(k)}\right)^{-1} e_{b_{2 k-1}+l}+j-b_{l}
\end{aligned}
$$

because $T^{j-b_{l}} e_{b_{l}}=\left(\prod_{i=1}^{j-b_{l}} w_{b_{l}+i}\right) e_{j}$ and $T^{j-b_{l}} e_{b_{2^{k-1}+l}}=\left(\prod_{i=1}^{j-b_{l}} w_{i}^{(k)}\right) e_{b_{2^{k-1}+l}+j-b_{l}}$.

Moreover, if $0 \leq m^{\prime} \leq \alpha m / 2$, then

$$
T^{m^{\prime}} e_{b_{2^{k-1}+l}+j-b_{l}}=\left(\prod_{i=j-b_{l}+1}^{j-b_{l}+m^{\prime}} w_{i}^{(k)}\right) e_{b_{2^{k-1}+l}+j-b_{l}+m^{\prime}},
$$

because $j-b_{l}+m^{\prime}<b_{l+1}-b_{l}+\alpha m / 2<\Delta^{\left(k_{0}\right)}+m / 2<m<\Delta^{(k)}$. So we get

$$
\begin{aligned}
T^{m+m^{\prime}} e_{b_{2^{k-1}+l+1}-m+j-b_{l}}= & \left(v^{(k)} \prod_{i=\Delta^{(k)}-m+j-b_{l}+1}^{\Delta^{(k)}-1} w_{i}^{(k)}\right)\left(\prod_{i=1}^{j-b_{l}} w_{b_{l}+i}\right) T^{m^{\prime}} e_{j} \\
& -\left(\prod_{i=j-b_{l}+1}^{\Delta^{(k)}-m+j-b_{l}} w_{i}^{(k)}\right)^{-1}\left(\prod_{i=j-b_{l}+1}^{j-b_{l}+m^{\prime}} w_{i}^{(k)}\right) e_{b_{2^{k-1}+l}+j-b_{l}+m^{\prime}}
\end{aligned}
$$

By definition of $z$, it follows that for any $0 \leq m^{\prime} \leq \alpha m / 2$, we have

$$
\begin{aligned}
& T^{m+m^{\prime}} z=T^{m^{\prime}} x-\sum_{l<2^{k_{0}}} \sum_{j=b_{l}}^{b_{l+1}-1}\left(v^{(k)} \prod_{i=j-b_{l}+m^{\prime}+1}^{\Delta^{(k)}-1} w_{i}^{(k)}\right)^{-1} . \\
&\left(\prod_{i=1}^{j-b_{l}} w_{b_{l}+i}\right)^{-1} x_{j} e_{b_{2}-1+l}+j-b_{l}+m^{\prime} .
\end{aligned}
$$

By (24) and since the weight sequence $w$ is bounded and bounded below, this implies that

$$
\left\|T^{m+m^{\prime}} z-T^{m^{\prime}} x\right\|<\|x\| \cdot C^{-1} \cdot A^{\Delta^{k_{0}}},
$$

where (as above) $A$ is an absolute constant. So we have $\left\|T^{m+m^{\prime}} z-T^{m^{\prime}} x\right\|<\varepsilon$ for every $0 \leq m^{\prime} \leq \alpha m / 2$ if $C$ is large enough.

The assumptions of Theorem 5.14 are thus satisfied, and this concludes the proof of Theorem 6.7.

Using Theorem 5.31 instead of Theorem 5.14, we can obtain sufficient conditions of the same kind as above for operators of $\mathrm{C}_{+}$-type to be frequently hypercyclic. 
Theorem 6.9. - Let $T=T_{v, w, \varphi, b}$ be an operator of $C_{+}$-type on $\ell_{p}(\mathbb{N})$. Suppose that there exists a constant $\alpha>0$ such that the following property holds true: for every $C \geq 1$ and every $k_{0} \geq 1$, there exist two integers $k \geq k_{0}$ and $1 \leq m<\Delta^{(k)}$ such that

$$
\left|v^{(k)}\right| \prod_{j=\Delta^{(k)}-m}^{\Delta^{(k)}-1}\left|w_{j}^{(k)}\right| \geq C
$$

and

$$
\left|v^{(k)}\right| \prod_{j=m^{\prime}+1}^{\Delta^{(k)}-1}\left|w_{j}^{(k)}\right|>C \quad \text { for every } 0 \leq m^{\prime} \leq \alpha \Delta^{(k)} .
$$

Then $T$ is chaotic and frequently hypercyclic.

Proof. - The proof of Theorem 6.9 is so similar to that of Theorem 6.7 that we only sketch it very briefly. The role of the integer $d$ in the assumption of Theorem 5.31 is played by the integer $2 \Delta^{(k)}$, which is a period of $z$. If $k$ is chosen sufficiently large at the beginning of the proof, $\Delta^{(k)}$ can be supposed to be a multiple of the period of any fixed vector $y \in X_{0}$ : indeed, such a vector always has a period of the form $\Delta^{\left(k_{1}\right)}$ for some integer $k_{1} \geq 1$, and $2 \Delta^{\left(k_{1}\right)}$ divides $\Delta^{(k)}$ as soon as $k>k_{1}$ since $\Delta^{(k)}=b_{2^{k-1}+2^{k_{1}-1}+1}-b_{2^{k-1}+2^{k_{1}-1}}$, $\Delta^{\left(k_{1}\right)}=b_{2^{k_{1}-1}+1}-b_{2^{k_{1}-1}}$ and $\varphi\left(2^{k-1}+2^{k_{1}-1}\right)=2^{k_{1}-1}$.

Now that we have obtained sufficient conditions for the frequent and $\mathcal{U}$-frequent hypercyclicity of operators of $\mathrm{C}_{+}$-type, we need to find necessary conditions as well. This we do in the next subsection.

6.3. Operators of C-type: how not to be FHC or UFHC. - Since $\mathcal{U}$-frequent hypercyclicity and frequent hypercyclicity are strong notions of hypercyclicity, one might think that it is easier to prove that an operator $T \in \mathfrak{B}(\mathcal{H})$ does not have one of these properties, than to prove that an operator has it. However, instead of exhibiting a single $\mathcal{U}$-frequent or frequent hypercyclic vector, we now need to prove that no vector of $\mathcal{H}$ whatsoever can be $\mathcal{U}$-frequent or frequent hypercyclic; and put in this way, this no longer looks that easy.

In this subsection, we are going to single out some conditions ensuring that an operator of $\mathrm{C}$-type $T$ is not $\mathcal{U}$-frequently hypercyclic or not frequently hypercyclic. As suggested in the few lines above, the arguments will be rather more technical than in the previous subsection. However, we can give the basic idea immediately: if for every hypercyclic vector $x$ for $T$, we are able to find some $\varepsilon>0$ such that the set $\mathcal{N}_{T}(x, B(0, \varepsilon))$ has upper density (resp. lower density) equal to 0 , then $T$ will not be $\mathcal{U}$-frequently hypercyclic (resp. frequently hypercyclic).

6.3.1. The main criterion, in abstract form. - The following notation will be used throughout this subsection: given an operator of C-type $T=T_{v, w, \varphi, b}$, we denote for each $n \geq 0$ by $P_{n}$ the canonical projection of $\ell_{p}(\mathbb{N})$ onto $E_{n}=\operatorname{span}\left[e_{k}, b_{n} \leq k<b_{n+1}\right]$ : if $x=\sum_{k \geq 0} x_{k} e_{k} \in \ell_{p}(\mathbb{N})$, then

$$
P_{n} x=\sum_{k=b_{n}}^{b_{n+1}-1} x_{k} e_{k}
$$


The following theorem provides sufficient conditions for an operator of C-type to be non- $\mathcal{U}$-frequently hypercyclic or non-frequently hypercyclic. These conditions are stated in terms of the projections $P_{n}$.

Theorem 6.10. - Let $T$ be an operator of $C$-type on $\ell_{p}(\mathbb{N})$. Suppose that for every hypercyclic vector $x \in \ell_{p}(\mathbb{N})$ for $T$, there exist

- a positive constant $C$,

- a non-increasing sequence $\left(\beta_{l}\right)_{l \geq 1}$ of positive real numbers with $\sum_{l \geq 1} \sqrt{\beta_{l}} \leq 1$,

- a sequence $\left(X_{l}\right)_{l \geq 0}$ of non-negative real numbers,

- a non-decreasing sequence $\left(N_{l}\right)_{l \geq 1}$ of integers tending to infinity,

such that the following conditions are satisfied:

(1) $\left\|P_{n} x\right\| \leq X_{n}$ for every $n \geq 0$;

(2) $\sup \left\|P_{n} T^{j} P_{l} x\right\| \leq C \beta_{l} X_{l}$ for every $l \geq 1$ and every $0 \leq n<l$; $j \geq 0$

(3) $\sup _{0 \leq j \leq N_{l}}\left\|P_{n} T^{j} P_{l} x\right\| \leq C \beta_{l}\left\|P_{l} x\right\|$ for every $l \geq 1$ and every $0 \leq n<l$;

(C) $\liminf _{l \rightarrow \infty} \inf _{k \geq N_{l}} \frac{\#\left\{0 \leq j \leq k ;\left\|P_{l} T^{j} P_{l} x\right\| \geq 2 C X_{l}\right\}}{k+1}=1$.

Then $T$ is not $\mathcal{U}$-frequently hypercyclic.

If Condition (C) is replaced by

(C') $\liminf _{l \rightarrow \infty} \inf _{k \geq N_{\min \left(\varphi^{-1}(l)\right)}} \frac{\#\left\{0 \leq j \leq k ;\left\|P_{l} T^{j} P_{l} x\right\| \geq 2 C X_{l}\right\}}{k+1}=1$,

then $T$ is not frequently hypercyclic.

The usefulness of this result lies in the fact that the lower bounds for the densities are given in terms of norms $\left\|P_{n} T^{j} P_{n} x\right\|$ which are easily computable. Moreover, since $P_{n} x$ is periodic, we can determine the cardinality of the sets $\left\{0 \leq j \leq k ;\left\|P_{n} T^{j} P_{n} x\right\| \geq 2 C X_{n}\right\}$ by examining only a fixed finite number of iterates (independent of $k$ ).

Theorem 6.10 will follow very easily from the next lemma, which provides, under conditions (1), (2), and (3) above on the projections $P_{n}$, lower bounds for the upper and lower density of some sets $\mathcal{N}_{T}\left(x, B(0, \varepsilon)^{c}\right)$, where $x$ is any non-zero vector of $\ell_{p}(\mathbb{N})$ and $\varepsilon$ is a positive number depending on $x$. Here, of course, $B(0, \varepsilon)^{c}$ denotes the complement of the the ball $B(0, \varepsilon)$ in $\ell_{p}(\mathbb{N})$.

Lemma 6.11. - Let $T$ be an operator of C-type on $\ell_{p}(\mathbb{N})$. Fix $x \in \ell_{p}(\mathbb{N}) \backslash\{0\}$ and suppose that there exist

- a positive constant $C$,

- a non-increasing sequence $\left(\beta_{l}\right)_{l \geq 1}$ of positive real numbers with $\sum_{l \geq 1} \sqrt{\beta_{l}} \leq 1$,

- a sequence $\left(X_{l}\right)_{l \geq 0}$ of non-negative real numbers,

- a non-decreasing sequence $\left(N_{l}\right)_{l \geq 1}$ of integers tending to infinity,

such that the following conditions are satisfied:

(1) $\left\|P_{n} x\right\| \leq X_{n}$ for every $n \geq 0$;

(2) $\sup _{j \geq 0}\left\|P_{n} T^{j} P_{l} x\right\| \leq C \beta_{l} X_{l}$ for every $l \geq 1$ and every $0 \leq n<l$;

(3) $\sup _{0 \leq j \leq N_{l}}\left\|P_{n} T^{j} P_{l} x\right\| \leq C \beta_{l}\left\|P_{l} x\right\|$ for every $l \geq 1$ and every $0 \leq n<l$; $0 \leq j \leq N_{l}$ 
(4) $\sup _{j \geq 0} \sum_{l>n}\left\|P_{n} T^{j} P_{l} x\right\|>C X_{n}$ for every $n \geq 0$.

Then there exists $\varepsilon>0$ such that

$$
\underline{\operatorname{dens}} \mathcal{N}_{T}\left(x, B(0, \varepsilon)^{c}\right) \geq \liminf _{l \rightarrow \infty} \inf _{k \geq N_{l}} \frac{\#\left\{0 \leq j \leq k ;\left\|P_{l} T^{j} P_{l} x\right\| \geq 2 C X_{l}\right\}}{k+1}
$$

and

$$
\overline{\operatorname{dens}} \mathcal{N}_{T}\left(x, B(0, \varepsilon)^{c}\right) \geq \liminf _{l \rightarrow \infty} \inf _{k \geq N_{\min \left(\varphi^{-1}(l)\right)}} \frac{\#\left\{0 \leq j \leq k ;\left\|P_{l} T^{j} P_{l} x\right\| \geq 2 C X_{l}\right\}}{k+1} .
$$

Let us show how this lemma implies Theorem 6.10.

Proof of Theorem 6.10, - For every $n \geq 0$ and every $x \in \ell_{p}(\mathbb{N})$, the vector $\sum_{0 \leq l \leq n} P_{l} x$ is a periodic vector for $T$. Hence, if $x$ is hypercyclic for $T$ then

$$
\sup _{j \geq 0} \sum_{l>n}\left\|P_{n} T^{j} P_{l} x\right\|=\infty .
$$

Indeed, otherwise we would have

$$
\sup _{j \geq 0}\left\|P_{n} T^{j} x\right\| \leq \sup _{j \geq 0}\left\|T^{j}\left(\sum_{0 \leq l \leq n} P_{l} x\right)\right\|+\sup _{j \geq 0} \sum_{l>n}\left\|P_{n} T^{j} P_{l} x\right\|<\infty,
$$

a contradiction with the hypercyclicity of $x$.

By (27), condition (4) of Lemma 6.11 is satisfied for any choice of the sequence $\left(X_{n}\right)_{n \geq 0}$. It follows that if condition (C) in Theorem 6.10 is satisfied, then, for any $x \in H C(T)$, one can find $\varepsilon>0$ such that $\overline{\operatorname{dens}}\left(\mathcal{N}_{T}(x, B(0, \varepsilon))=0\right.$; whereas if (C') is satisfied, then, for any $x \in H C(T)$, one can find $\varepsilon>0$ such that dens $\left(\mathcal{N}_{T}(x, B(0, \varepsilon))=0\right.$. This concludes the proof.

Before giving the proof of Lemma 6.11, let us explain the general idea. The starting point is given by the inequalities

$$
\left\|T^{j} x\right\| \geq\left\|P_{n} T^{j} x\right\| \geq\left\|P_{n} T^{j} P_{n} x\right\|-\sum_{l>n}\left\|P_{n} T^{j} P_{l} x\right\| .
$$

In other words, we get a lower bound for $\left\|T^{j} x\right\|$ as soon as we have a lower bound for some quantity $\left\|P_{n} T^{j} P_{n} x\right\|$ and an upper bound for $\left\|P_{n} T^{j} P_{l} x\right\|, l>n$. The role of the lower bound for $\left\|P_{n} T^{j} P_{n} x\right\|$ will be played by $2 C X_{n}$, which will be bigger than $2 \varepsilon$. Assumption (3) will provide us with an upper bound for $\left\|P_{n} T^{j} P_{l} x\right\|$ when $j$ is smaller than $N_{l}$. However, condition (4) tells us that $\left\|P_{n} T^{j} P_{l} x\right\|$ will be large for some $j \geq 0$ and some $l>n$. Assumption (2) will then be used to deduce that $X_{l} \geq X_{n}$, which will allow us to deduce that $2 C X_{l} \geq 2 \varepsilon$ and to repeat the above arguments for $\left\|P_{l} T^{j} x\right\|$, and so on.

Proof of Lemma 6.11. - If $x$ is a non-zero periodic vector for $T$, one can obviously choose $\varepsilon>0$ such that

$$
\underline{\operatorname{dens}} \mathcal{N}_{T}\left(x, B(0, \varepsilon)^{c}\right)=1
$$

and the conclusion of Lemma 6.11 is satisfied. So we henceforward suppose that $x$ is not a periodic vector for $T$.

We first note that there exists an integer $l_{0} \geq 1$ such that

$$
\left\|P_{l_{0}} x\right\| \geq \sqrt{\beta_{l_{0}}}\left\|x-P_{0} x\right\| .
$$


Indeed, since $\sum_{l \geq 1} \sqrt{\beta_{l}} \leq 1$ it is impossible to have $\left\|P_{l} x\right\|<\sqrt{\beta_{l}}\left\|x-P_{0} x\right\|$ for every $l \geq 1$. Since $x$ is not periodic, $x-P_{0} x$ is non-zero. Assumption (1) of Lemma 6.11implies thus that $X_{l_{0}}$ is non-zero and we set $\varepsilon=C X_{l_{0}}$.

We now construct, by induction on $n \geq 1$, a strictly increasing sequence of integers $\left(l_{n}\right)_{n \geq 1}$ such that if we set

then

$$
j_{n-1}:=\min \left\{j \geq 0 ; \sum_{l>l_{n-1}}\left\|P_{l_{n-1}} T^{j} P_{l} x\right\|>C X_{l_{n-1}}\right\},
$$

$$
j_{n-1}>N_{l_{n}} \quad \text { and } \quad X_{l_{n}} \geq \frac{1}{\sqrt{\beta_{l_{n}}}} X_{l_{n-1}} \quad \text { for every } n \geq 1 .
$$

Observe that this does make sense: the set involved in the definition of $j_{n-1}$ is non-empty by assumption (4).

Suppose that the integers $l_{1}, \ldots, l_{n-1}$ have already been constructed. Then there exists an integer $l_{n}>l_{n-1}$ with the property that

$$
\left\|P_{l_{n-1}} T^{j_{n-1}} P_{l_{n}} x\right\|>C \sqrt{\beta_{l_{n}}} X_{l_{n-1}} .
$$

Indeed, if we had $\left\|P_{l_{n-1}} T^{j_{n-1}} P_{l} x\right\| \leq C \sqrt{\beta_{l}} X_{l_{n-1}}$ for every $l>l_{n-1}$, this would imply that $\sum_{l>l_{n-1}}\left\|P_{l_{n-1}} T^{j_{n-1}} P_{l} x\right\| \leq C X_{l_{n-1}}$, violating the definition of $j_{n-1}$.

By assumption (2), we have

$$
X_{l_{n}} \geq \frac{1}{C \beta_{l_{n}}}\left\|P_{l_{n-1}} T^{j_{n-1}} P_{l_{n}} x\right\| \geq \frac{1}{\sqrt{\beta_{l_{n}}}} X_{l_{n-1}} .
$$

In order to show that $j_{n-1}>N_{l_{n}}$, we observe that for every $0 \leq j \leq N_{l_{n}}$,

$$
\left\|P_{l_{n-1}} T^{j} P_{l_{n}} x\right\| \leq C \beta_{l_{n}}\left\|P_{l_{n}} x\right\| \leq C \beta_{l_{n}}\left\|x-P_{0} x\right\| \leq \frac{C \beta_{l_{n}}}{\sqrt{\beta_{l_{0}}}}\left\|P_{l_{0}} x\right\| \leq \frac{C \beta_{l_{n}}}{\sqrt{\beta_{l_{0}}}} X_{l_{0}},
$$

by assumption (3) and the definition of $l_{0}$. Since the sequence $\left(\beta_{l}\right)_{l \geq 0}$ is decreasing, this yields that $\left\|P_{l_{n-1}} T^{j} P_{l_{n}} x\right\| \leq C \sqrt{\beta_{l_{n}}} X_{l_{0}}$. Also, the induction hypothesis implies that $X_{l_{0}} \leq X_{l_{1}} \leq \cdots \leq X_{l_{n}}$, so that $\left\|P_{l_{n-1}} T^{j} P_{l_{n}} x\right\| \leq C \sqrt{\beta_{l_{n}}} X_{l_{n-1}}$ for every $0 \leq j \leq N_{l_{n}}$. Inequality (28) thus implies that $j_{n-1}>N_{l_{n}}$ and this finishes the induction. Note that the sequence $\left(j_{n}\right)_{n \geq 0}$ tends to infinity as $n$ tends to infinity.

For any integer $s \geq 1$, let us denote by $n_{s}$ be the smallest integer such that $s$ belongs to $\left[j_{n_{s}-1}, j_{n_{s}}\right)$. Then $n_{s}$ tends to infinity as $s$ tends to infinity. Since

for every $j \geq 0$ and $n \geq 0$, we have

$$
\left\|T^{j} x\right\| \geq\left\|P_{n} T^{j} x\right\| \geq\left\|P_{n} T^{j} P_{n} x\right\|-\sum_{l>n}\left\|P_{n} T^{j} P_{l} x\right\|
$$

$$
\left\|T^{j} x\right\| \geq\left\|P_{l_{n}} T^{j} P_{l_{n}} x\right\|-C X_{l_{n}} \quad \text { for every } 0 \leq j<j_{n} .
$$

It follows from this inequality and the fact that $j<j_{n_{s}}$ for every $j<s$ that

$$
\begin{aligned}
\left\{0 \leq j \leq s ;\left\|P_{l_{n_{s}}} T^{j} P_{l_{n_{s}}} x\right\| \geq 2 C X_{l_{n_{s}}}\right\} & \subseteq\left\{0 \leq j \leq s ;\left\|T^{j} x\right\| \geq C X_{l_{n_{s}}}\right\} \\
& \subseteq\left\{0 \leq j \leq s ;\left\|T^{j} x\right\| \geq C X_{l_{0}}\right\} .
\end{aligned}
$$

Hence, since $\varepsilon=C X_{l_{0}}$, we have

$$
\underline{\operatorname{dens}} \mathcal{N}_{T}\left(x, B(0, \varepsilon)^{c}\right) \geq \liminf _{s \rightarrow \infty} \frac{\#\left\{0 \leq j \leq s ;\left\|P_{l_{n_{s}}} T^{j} P_{l_{n_{s}}} x\right\| \geq 2 C X_{l_{n_{s}}}\right\}}{s+1} .
$$


Now $N_{l_{s}}<j_{n_{s}-1} \leq s$, so that $s$ belongs to the interval $\left[N_{l_{s}}, \infty\right)$ for each $s \geq 1$. Thus

$$
\begin{aligned}
& \frac{\#\left\{0 \leq j \leq s ;\left\|P_{n_{s}} T^{j} P_{l_{s}} x\right\| \geq 2 C X_{l_{n_{s}}}\right\}}{s+1} \geq \\
& \inf _{k \geq N_{l_{s}}} \frac{\#\left\{0 \leq j \leq k ;\left\|P_{l_{n_{s}}} T^{j} P_{l_{n_{s}}} x\right\| \geq 2 C X_{l_{n_{s}}}\right\}}{k+1}
\end{aligned}
$$

and

$$
\begin{aligned}
\text { dens } \mathcal{N}_{T}\left(x, B(0, \varepsilon)^{c}\right) & \geq \liminf _{s \rightarrow \infty} \inf _{k \geq N_{l_{s}}} \frac{\#\left\{0 \leq j \leq k ;\left\|P_{l_{n_{s}}} T^{j} P_{l_{n_{s}}} x\right\| \geq 2 C X_{l_{n_{s}}}\right\}}{k+1} \\
& \geq \liminf _{l \rightarrow \infty} \inf _{k \geq N_{l}} \frac{\#\left\{0 \leq j \leq k ;\left\|P_{l} T^{j} P_{l} x\right\| \geq 2 C X_{l}\right\}}{k+1}
\end{aligned}
$$

since $l_{n_{s}} \rightarrow \infty$ as $s \rightarrow \infty$. This proves the first part of Lemma 6.11.

As for the second part, we proceed in the same way, starting from the inequality

$$
\overline{\operatorname{dens}} \mathcal{N}_{T}\left(x, B(0, \varepsilon)^{c}\right) \geq \limsup _{s \rightarrow \infty} \frac{\#\left\{0 \leq j \leq s ;\left\|P_{l_{n_{s}}} T^{j} P_{l_{n_{s}}} x\right\| \geq 2 C X_{l_{n_{s}}}\right\}}{s+1} .
$$

Our first observation is that $\min \left(\varphi^{-1}\left(l_{n}\right)\right) \leq l_{n+1}$ for every $n \geq 1$. This follows, in a slightly roundabout way, from the fact that $P_{l_{n}} T^{j_{n}} P_{l_{n+1}}$ is non-zero. Indeed, the definition of $T$ implies that there exists an integer $i \geq 1$ such that $\varphi^{i}\left(l_{n+1}\right)=l_{n}$. Then $\varphi^{i-1}\left(l_{n+1}\right)$ belongs to $\varphi^{-1}\left(l_{n}\right)$, and since $\varphi^{i-1}\left(l_{n+1}\right) \leq l_{n+1}$, it follows that $\min \left(\varphi^{-1}\left(l_{n}\right)\right) \leq l_{n+1}$.

We now claim that there exists a strictly increasing sequence of integers $\left(s_{i}\right)_{i \geq 1}$ such that $s_{i} \geq N_{\min \left(\varphi^{-1}\left(l_{n_{s_{i}}}\right)\right)}$ for every $i \geq 1$. Recall that $N_{l_{n_{i}+1}}<j_{n_{i}}$ for all $i \geq 1$. Thus there exists $s_{i} \in\left[j_{n_{i}-1}, j_{n_{i}}\right)$ such that $s_{i} \geq N_{l_{n_{i}+1}}$. As $s_{i}$ belongs to the interval $\left[j_{n_{i}-1}, j_{n_{i}}\right)$, we have $n_{s_{i}} \leq n_{i}$, and thus $s_{i} \geq N_{l_{n_{i}+1}} \geq N_{\min \left(\varphi^{-1}\left(l_{n_{s_{i}}}\right)\right)}$. Extracting if necessary in order to make the sequence $\left(s_{i}\right)_{i \geq 1}$ strictly increasing, we obtain the sequence we are looking for.

We now have

$$
\begin{aligned}
\overline{\operatorname{dens}} \mathcal{N}_{T}\left(x, B(0, \varepsilon)^{c}\right) \geq \liminf _{i \rightarrow \infty} \frac{\#\left\{0 \leq j \leq s_{i} ;\left\|P_{l_{s_{i}}} T^{j} P_{l_{s_{i}}} x\right\| \geq 2 C X_{l_{n_{s_{i}}}}\right\}}{s_{i}+1} \\
\geq \liminf _{n \rightarrow \infty} \inf _{k \geq N_{\min \left(\varphi^{-1}\left(l_{n}\right)\right)}} \frac{\#\left\{0 \leq j \leq k ;\left\|P_{l_{n}} T^{j} P_{l_{n}} x\right\| \geq 2 C X_{l_{n}}\right\}}{k+1} \\
\geq \liminf _{l \rightarrow \infty} \inf _{k \geq N_{\min \left(\varphi^{-1}(l)\right)}} \frac{\#\left\{0 \leq j \leq k ;\left\|P_{l} T^{j} P_{l} x\right\| \geq 2 C X_{l}\right\}}{k+1} .
\end{aligned}
$$

This concludes the proof of Lemma 6.11,

6.3.2. How to check the assumptions. - The assumptions of Theorem 6.10 are partly of an "abstract" nature, since they involve the projections $P_{n}$ and not explicitly the parameters $v, w, \varphi$, and $b$ defining $T$. We now provide concrete conditions on $v, w, \varphi$, and $b$ which imply that the operator of C-type $T_{v, w, \varphi, b}$ satisfies the assumptions of Theorem 6.10,

As a rule, conditions (1), (2), and (3) will be obtained by requiring that the products of weights

$$
\left|v_{n}\right| \cdot \sup _{j \in\left[b_{\varphi(n)}, b_{\varphi(n)+1}\right)} \prod_{s=b_{\varphi(n)}+1}^{j}\left|w_{s}\right|
$$


decrease sufficiently rapidly and by setting, given a hypercyclic vector $x$ for $T_{v, w, \varphi, b}$,

$$
X_{l}:=\left\|\sum_{k=b_{l}}^{b_{l+1}-1}\left(\prod_{s=k+1}^{b_{l+1}-1} w_{s}\right) x_{k} e_{k}\right\|, \quad l \geq 0 .
$$

More precisely, we have the following fact, which generalizes Claims 5 and 6 in $[\mathbf{3 9}$.

Fact 6.12. - Let $T=T_{v, w, \varphi, b}$ be an operator of C-type on $\ell_{p}(\mathbb{N})$, and let $\left(C_{n}\right)_{n \geq 0}$ be a sequence of positive numbers with $0<C_{n}<1$. Assume that

$$
\left|v_{n}\right| \cdot \sup _{j \in\left[b_{\varphi(n)}, b_{\varphi(n)+1}\right)}\left(\prod_{s=b_{\varphi(n)}+1}^{j}\left|w_{s}\right|\right) \leq C_{n} \quad \text { for every } n \geq 0 .
$$

Then, for any $x \in \ell_{p}(\mathbb{N})$, we have for every $l \geq 1$ and every $0 \leq n<l$,

(1) $\sup _{j \geq 0}\left\|P_{n} T^{j} P_{l} x\right\| \leq C_{l}\left(b_{l+1}-b_{l}\right)^{\frac{p-1}{p}}\left\|\sum_{k=b_{l}}^{b_{l+1}-1}\left(\prod_{s=k+1}^{b_{l+1}-1} w_{s}\right) x_{k} e_{k}\right\|$

and

$$
\sup _{j \leq N}\left\|P_{n} T^{j} P_{l} x\right\| \leq C_{l}\left(b_{l+1}-b_{l}\right)^{\frac{p-1}{p}}\left(\sup _{b_{l+1}-N \leq k<b_{l+1}} \prod_{s=k+1}^{b_{l+1}-1}\left|w_{s}\right|\right)\left\|P_{l} x\right\|
$$

for every $1 \leq N \leq b_{l+1}-b_{l}$.

Proof. - Fix $l \geq 1$ and $0 \leq n<l$. We first remark that if $n \neq \varphi^{M}(l)$ for all $M \geq 1$, the definition of $T$ implies that $P_{n} T^{j} P_{l} x=0$ for every $j \geq 0$ (this argument was already used at the end of the proof of Lemma 6.11). So the required inequalities are obvious in this case.

Suppose now that $n=\varphi^{M}(l)$ for some integer $M \geq 1$. In this case, we claim that

$$
\sup _{j \geq 0}\left\|P_{n} T^{j} e_{k}\right\| \leq\left|v_{l}\right|\left(\prod_{s=k+1}^{b_{l+1}-1}\left|w_{s}\right|\right) \sup _{j \geq 0}\left\|P_{n} T^{j} e_{b_{\varphi(l)}}\right\|
$$

for every $k \in\left[b_{l}, b_{l+1}\right)$. This inequality will allow us to run an induction procedure in order to estimate the quantity $\sup _{j \geq 0}\left\|P_{n} T^{j} P_{l} x\right\|$.

In order to prove (29), we fix $j_{0} \geq 0$ and we consider separately several cases.

- Suppose first that $0 \leq j_{0}<b_{l+1}-k$. Then $T^{j_{0}} e_{k}$ is a scalar multiple of $e_{k+j_{0}}$, and $P_{n} T^{j_{0}} e_{k}=0$.

- Now, suppose that $b_{l+1}-k \leq j_{0}<b_{l+1}-k+b_{l+1}-b_{l}$. Then

so that

$$
P_{n} T^{j_{0}} e_{k}=P_{n} T^{j_{0}-\left(b_{l+1}-k\right)}\left(v_{l}\left(\prod_{s=k+1}^{b_{l+1}-1} w_{s}\right) e_{b_{\varphi(l)}}\right)
$$

$$
\left\|P_{n} T^{j_{0}} e_{k}\right\| \leq\left|v_{l}\right|\left(\prod_{s=k+1}^{b_{l+1}-1}\left|w_{s}\right|\right) \sup _{j \geq 0}\left\|P_{n} T^{j} e_{b_{\varphi(l)}}\right\| .
$$

- Finally, suppose that $b_{l+1}-k+b_{l+1}-b_{l} \leq j_{0}<2\left(b_{l+1}-b_{l}\right)$. In this case, we write

$$
T^{j_{0}} e_{k}=\left(\prod_{s=b_{l}+1}^{k} w_{s}\right)^{-1} T^{j_{0}+k-b_{l}} e_{b_{l}} .
$$


By assumption, we have $2\left(b_{l+1}-b_{l}\right) \leq j_{0}+k-b_{l}<2\left(b_{l+1}-b_{l}\right)+\left(b_{l+1}-b_{l}\right)$; that is,

$$
j_{0}+k-b_{l}=i+2\left(b_{l+1}-b_{l}\right) \quad \text { with } 0 \leq i<b_{l+1}-b_{l} .
$$

Since $T^{2\left(b_{l+1}-b_{l}\right)} e_{b_{l}}=e_{b_{l}}$, it follows that

$$
T^{j_{0}} e_{k}=\left(\prod_{s=b_{l}+1}^{k} w_{s}\right)^{-1} T^{i} e_{b_{l}}=\left(\prod_{s=b_{l}+1}^{k} w_{s}\right)^{-1}\left(\prod_{s=b_{l}+1}^{l+i} w_{s}\right) e_{b_{l}+i}
$$

and hence that $P_{n} T^{j_{0}} e_{k}=0$.

We have thus proved the required inequality for every $0 \leq j_{0}<2\left(b_{l+1}-b_{l}\right)$. Since $T^{2\left(b_{l+1}-b_{l}\right)} e_{k}=e_{k}$, it holds true in fact for every $j \geq 0$, and this proves (29).

Let now $M_{0}$ be the minimum of the integers $M \geq 1$ such that $\varphi^{M}(l)=n$. We start from the straightforward estimate

$$
\sup _{j \geq 0}\left\|P_{n} T^{j} P_{l} x\right\| \leq \sum_{k=b_{l}}^{b_{l+1}-1}\left|x_{k}\right| \sup _{j \geq 0}\left\|P_{n} T^{j} e_{k}\right\|
$$

and apply (29). This gives

$$
\begin{aligned}
\sup _{j \geq 0}\left\|P_{n} T^{j} P_{l} x\right\| & \leq \sum_{k=b_{l}}^{b_{l+1}-1}\left|x_{k}\right| \cdot\left|v_{l}\right| \cdot\left(\prod_{s=k+1}^{b_{l+1}-1}\left|w_{s}\right|\right) \sup _{j \geq 0}\left\|P_{n} T^{j} e_{b_{\varphi(l)}}\right\| \\
& =\left|v_{l}\right| \cdot \sup _{j \geq 0}\left\|P_{n} T^{j} e_{b_{\varphi(l)}}\right\| \cdot\left\|\sum_{k=b_{l}}^{b_{l+1}-1}\left(\prod_{s=k+1}^{b_{l+1}-1} w_{s}\right) x_{k} e_{k}\right\|_{1},
\end{aligned}
$$

where $\|z\|_{1}$ denotes the $\ell^{1}$ norm of a vector $z \in c_{00}$.

By induction, we obtain

$$
\begin{aligned}
\sup _{j \geq 0}\left\|P_{n} T^{j} P_{l} x\right\| \leq\left|v_{l}\right| \cdot \prod_{r=1}^{M_{0}-1}\left(\left|v_{\varphi^{r}(l)}\right| \cdot \prod_{s=b_{\varphi^{r}(l)}+1}^{b_{\varphi^{r}(l)+1}-1}\left|w_{s}\right|\right) \cdot \\
\sup _{j \geq 0}\left\|P_{n} T^{j} e_{b_{n}}\right\| \cdot\left\|\sum_{k=b_{l}}^{b_{l+1}-1}\left(\prod_{s=k+1}^{b_{l+1}-1} w_{s}\right) x_{k} e_{k}\right\|_{1} \\
=\prod_{r=1}^{M_{0}-1}\left(\left|v_{\varphi^{r-1}(l)}\right| \prod_{s=b_{\varphi^{r}(l)}+1}^{b_{\varphi^{r}(l)+1}-1}\left|w_{s}\right|\right) \cdot\left|v_{\varphi^{M_{0}-1}(l)}\right| \\
\sup _{j \geq 0}\left\|P_{n} T^{j} e_{b_{n}}\right\| \cdot\left\|\sum_{k=b_{l}}^{b_{l+1}-1}\left(\prod_{s=k+1}^{b_{l+1}-1} w_{s}\right) x_{k} e_{k}\right\|_{1} .
\end{aligned}
$$

Applying the assumption of Fact 6.12, it follows that

$$
\begin{gathered}
\sup _{j \geq 0}\left\|P_{n} T^{j} P_{l} x\right\| \leq\left(\prod_{r=1}^{M_{0}-1} C_{\varphi^{r-1}(l)}\right) \cdot\left|v_{\varphi^{M_{0}-1}(l)}\right| \cdot \sup _{j \geq 0}\left\|P_{n} T^{j} e_{b_{n}}\right\| \cdot \\
\left\|\sum_{k=b_{l}}^{b_{l+1}-1}\left(\prod_{s=k+1}^{b_{l+1}-1} w_{s}\right) x_{k} e_{k}\right\|_{1} .
\end{gathered}
$$


Now, the definition of $T$ and the fact that $T^{2\left(b_{n+1}-b_{n}\right)} e_{b_{n}}=e_{b_{n}}$ show that

$$
\sup _{j \geq 0}\left\|P_{n} T^{j} e_{b_{n}}\right\| \leq \sup _{j \in\left[b_{n}, b_{n+1}\right)} \prod_{s=b_{n}+1}\left|w_{s}\right| .
$$

Since $n=\varphi^{M_{0}}(l)$, it follows (by the assumption of Fact 6.12), that

$$
\begin{aligned}
\sup _{j \geq 0}\left\|P_{n} T^{j} P_{l} x\right\| & \leq\left(\prod_{r=1}^{M_{0}-1} C_{\varphi^{r-1}(l)}\right) \cdot C_{\varphi^{M_{0}-1}(l)} \cdot\left\|\sum_{k=b_{l}}^{b_{l+1}-1}\left(\prod_{s=k+1}^{b_{l+1}-1} w_{s}\right) x_{k} e_{k}\right\|_{1} \\
& \leq C_{l} \cdot\left\|\sum_{k=b_{l}}^{b_{l+1}-1}\left(\prod_{s=k+1}^{b_{l+1}-1} w_{s}\right) x_{k} e_{k}\right\|_{1},
\end{aligned}
$$

because all the constants $C_{n}$ are supposed to belong to $(0,1)$ and $\varphi^{0}(l)=l$. By Hölder's inequality, we conclude that

$$
\sup _{j \geq 0}\left\|P_{n} T^{j} P_{l} x\right\| \leq C_{l} \cdot\left(b_{l+1}-b_{l}\right)^{1-\frac{1}{p}} \cdot\left\|\sum_{k=b_{l}}^{b_{l+1}-1}\left(\prod_{s=k+1}^{b_{l+1}-1} w_{s}\right) x_{k} e_{k}\right\|,
$$

which proves assertion (1) of Fact 6.12.

The proof of assertion (2) is now straightforward. Indeed, we have for every $1 \leq N \leq$ $b_{l+1}-b_{l}$ and every $0 \leq j \leq N$ :

$$
T^{j} P_{l} x=T^{j}\left(\sum_{k=b_{l}}^{b_{l+1}-N-1} x_{k} e_{k}\right)+T^{j}\left(\sum_{k=b_{l+1}-N}^{b_{l+1}-1} x_{k} e_{k}\right) \text {. }
$$

Since the first term belongs to the linear span of the vectors $e_{i}, b_{l} \leq i<b_{l+1}$, it follows that

$$
P_{n} T^{j} P_{l} x=P_{n} T^{j}\left(\sum_{k=b_{l+1}-N}^{b_{l+1}-1} x_{k} e_{k}\right),
$$

and so, by the already proved assertion (1) of Fact 6.12

$$
\begin{aligned}
\left\|P_{n} T^{j} P_{l} x\right\| & \leq C_{l} \cdot\left(b_{l+1}-b_{l}\right)^{1-\frac{1}{p}} \cdot\left\|\sum_{k=b_{l+1}-N}^{b_{l+1}-1}\left(\prod_{s=k+1}^{b_{l+1}-1} w_{s}\right) x_{k} e_{k}\right\| \\
& \leq C_{l} \cdot\left(b_{l+1}-b_{l}\right)^{1-\frac{1}{p}} \cdot \sup _{b_{l+1}-N \leq k<b_{l+1}}\left(\prod_{s=k+1}^{b_{l+1}-1}\left|w_{s}\right|\right) \cdot\left\|P_{l} x\right\| .
\end{aligned}
$$

This concludes the proof of Fact 6.12 ,

The next fact provides conditions on the parameters of an operator of C-type ensuring that assumption (C) or (C') of Theorem 6.10 is satisfied. This generalizes Claim 7 of [39].

Fact 6.13. - Let $T=T_{v, w, \varphi, b}$ be an operator of C-type on $\ell_{p}(\mathbb{N})$, and let $x \in \ell_{p}(\mathbb{N})$. Fix $l \geq 0$, and define

$$
X_{l}=\left\|\sum_{k=b_{l}}^{b_{l+1}-1}\left(\prod_{s=k+1}^{b_{l+1}-1} w_{s}\right) x_{k} e_{k}\right\|
$$

Suppose that there exist two integers $0 \leq k_{0}<k_{1} \leq b_{l+1}-b_{l}$ such that

$$
\left|w_{b_{l}+k}\right|=1 \quad \text { for every } k \in\left(k_{0}, k_{1}\right) \text { and } \prod_{s=b_{l}+k_{0}+1}^{b_{l+1}-1}\left|w_{s}\right|=1 \text {. }
$$


Then we have for every $J \geq 0$

$$
\begin{aligned}
\frac{1}{J+1} \#\{0 \leq j \leq & \left.J\left\|P_{l} T^{j} P_{l} x\right\| \geq X_{l} / 2\right\} \\
& \geq 1-2\left(b_{l+1}-b_{l}-\left(k_{1}-k_{0}\right)\right) \cdot\left(\frac{1}{J+1}+\frac{1}{b_{l+1}-b_{l}}\right) .
\end{aligned}
$$

Proof. - Fix $j \geq 0$ and $k \in\left[b_{l}, b_{l+1}\right)$, and set $n:=j+k-b_{l} \bmod \left(b_{l+1}-b_{l}\right)$. Then

$$
\begin{aligned}
P_{l} T^{j} e_{k} & = \pm\left(\prod_{s=k+1}^{b_{l+1}-1} w_{s}\right)\left(\prod_{s=b_{l}+1}^{b_{l}+n} w_{s}\right)\left(\prod_{s=b_{l}+1}^{b_{l+1}-1} w_{s}\right)^{-1} e_{b_{l}+n} \\
& = \pm\left(\prod_{s=k+1}^{b_{l+1}-1} w_{s}\right)\left(\prod_{s=b_{l}+n+1}^{b_{l+1}-1} w_{s}\right)^{-1} e_{b_{l}+n}
\end{aligned}
$$

(the plus or minus sign appearing in these equalities depends on the parity of the unique integer $s \geq 0$ such that $n$ belongs to the interval $\left.\left[s\left(b_{l+1}-b_{l}\right),(s+1)\left(b_{l+1}-b_{l}\right)\right)\right)$.

If we suppose that $n$ belongs to $\left[k_{0}, k_{1}\right)$, we have

$$
\prod_{s=b_{l}+n+1}^{b_{l+1}-1}\left|w_{s}\right|=\prod_{s=b_{l}+k_{0}+1}^{b_{l+1}-1}\left|w_{s}\right|=1
$$

and thus there exists $\zeta_{j, k} \in \mathbb{T}$ such that

$$
P_{l} T^{j} e_{k}=\zeta_{j, k} \cdot\left(\prod_{s=k+1}^{b_{l+1}-1} w_{s}\right) e_{b_{l}+n}
$$

Setting, for every $j \geq 0$,

$$
I_{j}:=\left\{k \in\left[b_{l}, b_{l+1}\right) ; j+k-b_{l} \bmod \left(b_{l+1}-b_{l}\right) \text { does not belong to }\left[k_{0}, k_{1}\right)\right\},
$$

we obtain that

$$
P_{l} T^{j} P_{l} x=\sum_{k=b_{l}}^{b_{l+1}-1} x_{k} P_{l} T^{j} e_{k}=\sum_{k \in\left[b_{l}, b_{l+1}\right) \backslash I_{j}} x_{k} P_{l} T^{j} e_{k}+\sum_{k \in I_{j}} x_{k} P_{l} T^{j} e_{k}
$$

satisfies

$$
\begin{aligned}
\left\|P_{l} T^{j} P_{l} x\right\| & \geq\left\|\sum_{k \in\left[b_{l}, b_{l+1}\right) \backslash I_{j}} x_{k} P_{l} T^{j} e_{k}\right\|=\left\|\sum_{k \in\left[b_{l}, b_{l+1}\right) \backslash I_{j}} x_{k} \zeta_{j, k}\left(\prod_{s=k+1}^{b_{l+1}-1} w_{s}\right) e_{k}\right\| \\
& =\left\|\sum_{k \in\left[b_{l}, b_{l+1}\right) \backslash I_{j}} x_{k}\left(\prod_{s=k+1}^{b_{l+1}-1} w_{s}\right) e_{k}\right\| \geq X_{l}-\left\|\sum_{k \in I_{j}} x_{k}\left(\prod_{s=k+1}^{b_{l+1}-1} w_{s}\right) e_{k}\right\| .
\end{aligned}
$$

We now consider separately two cases:

- Suppose first that $\left\|\sum_{k \in I_{j}} x_{k}\left(\prod_{s=k+1}^{b_{l+1}-1} w_{s}\right) e_{k}\right\|<X_{l} / 2$ for every $j \geq 0$. Then we have $\left\|P_{l} T^{j} P_{l} x\right\| \geq X_{l} / 2$ for every $j \geq 0$, and thus

$$
\frac{1}{J+1} \#\left\{0 \leq j \leq J ;\left\|P_{l} T^{j} P_{l} x\right\| \geq X_{l} / 2\right\}=1 \quad \text { for every } J \geq 0 .
$$


- Now, suppose that there exists $j_{0} \geq 0$ such that

$$
\left\|\sum_{k \in I_{j_{0}}} x_{k}\left(\prod_{s=k+1}^{b_{l+1}-1} w_{s}\right) e_{k}\right\| \geq \frac{X_{l}}{2} .
$$

Then we have for every integer $j \geq 0$ such that $I_{j} \cap I_{j_{0}}$ is empty:

$$
\left\|P_{l} T^{j} P_{l} x\right\| \geq\left\|\sum_{k \in\left[b_{l}, b_{l+1}\right) \backslash I_{j}}\left(\prod_{s=k+1}^{b_{l+1}-1} w_{s}\right) x_{k} e_{k}\right\| \geq\left\|\sum_{k \in I_{j_{0}}}\left(\prod_{s=k+1}^{b_{l+1}-1} w_{s}\right) x_{k} e_{k}\right\| \geq \frac{X_{l}}{2} .
$$

It follows that, for every $J \geq 0$,

$$
\begin{aligned}
\frac{1}{J+1} \#\left\{0 \leq j \leq J ;\left\|P_{l} T^{j} P_{l} x\right\|\right. & \left.\geq X_{l} / 2\right\} \\
\geq & 1-\frac{1}{J+1} \#\left\{0 \leq j \leq J ; I_{j} \cap I_{j_{0}} \neq \emptyset\right\} .
\end{aligned}
$$

Now, we remark that if we set $i_{j}:=j \bmod \left(b_{l+1}-b_{j}\right)$ for every $j \geq 0$, we have

$$
I_{j}= \begin{cases}{\left[b_{l}+k_{1}-i_{j}, b_{l+1}\right) \cup\left[b_{l}, k_{0}-i_{j}\right)} & \text { if } 0 \leq i_{j}<k_{0} \\ {\left[b_{l}+k_{1}-i_{j}, b_{l+1}+k_{0}-i_{j}\right)} & \text { if } k_{0} \leq i_{j} \leq k_{1} \\ {\left[b_{l}, b_{l+1}+k_{0}-i_{j}\right) \cup\left[b_{l+1}+k_{1}-i_{j}, b_{l+1}\right)} & \text { if } k_{1}<i_{j}<b_{l+1}-b_{l}\end{cases}
$$

and $\# I_{j}=b_{l+1}-b_{l}-\left(k_{1}-k_{0}\right)$ for every $j \geq 0$. This particular structure of the sets $I_{j}$ implies that

$$
\#\left\{0 \leq j \leq J ; I_{j} \cap I_{j_{0}} \neq \emptyset\right\} \leq 2\left(b_{l+1}-b_{l}-\left(k_{1}-k_{0}\right)\right) \cdot\left(\left\lfloor\frac{J}{b_{l+1}-b_{l}}\right\rfloor+1\right) .
$$

Plugging this into (30) yields that

$\frac{1}{J+1} \#\left\{0 \leq j \leq J ;\left\|P_{l} T^{j} P_{l} x\right\| \geq X_{l} / 2\right\} \geq 1-2\left(b_{l+1}-b_{l}-\left(k_{1}-k_{0}\right)\right)\left(\frac{1}{J+1}+\frac{1}{b_{l+1}-b_{l}}\right)$,

which is the inequality we were looking for.

Facts 6.12 and 6.13 will be repeatedly used in Subsections 6.5, 6.7 and 6.8 in order to exhibit the desired counterexamples.

6.4. Operators of C-type: mixing or not mixing. - In this subsection, we give some conditions ensuring that an operator of C-type is or is not topologically mixing. This will allow us to show in particular in Section 6.5.2 that the assumptions of our criterion for frequent hypercyclicity (Theorem 5.31) do not imply the Operator Specification Property.

Proposition 6.14. - Let $T=T_{v, w, \varphi, b}$ be an operator of C-type on $\ell_{p}(\mathbb{N})$. Suppose that for every $\eta>0$ and every $n \geq 0$, the set

$$
S_{\eta, n}:=\bigcup_{m \in \mathcal{N}_{\eta, n}}\left\{s \in\left[1, b_{m+1}-b_{m}\right):\left|v_{m}\right| \prod_{i=b_{m+1}-s}^{b_{m+1}-1}\left|w_{i}\right|>\frac{1}{\eta}\right\} \quad \text { is cofinite, }
$$

where $\mathcal{N}_{\eta, n}=\left\{m \in \varphi^{-1}(n):\left|v_{m}\right| \prod_{i=b_{m}+1}^{b_{m+1}-1}\left|w_{i}\right|>\frac{1}{\eta}\right\}$. Then $T$ is topologically mixing. 
Proof. - Since $T$ has a dense set of periodic points, Corollary 5.4 implies that it suffices to show that for every $k \geq 0$ and every $\varepsilon>0$, there exists an integer $M \geq 1$ such that for every $m \geq M$, there exists a vector $z \in \ell_{p}(\mathbb{N})$ such that $\|z\|<\varepsilon$ and $\left\|T^{m} z-e_{k}\right\|<\varepsilon$.

Fix thus $k \geq 0$ and $\varepsilon>0$. Let also $n \geq 0$ be such that $k$ belongs to $\left[b_{n}, b_{n+1}\right)$, and let $\eta>0$ be a sufficiently small positive number, to be fixed later on in the proof. By assumption, the set $S_{\eta, n}$ is cofinite. So it suffices to show that if $\eta$ is small enough, then, for every $s \in S_{\eta, n}$, one can find a vector $z_{s} \in \ell_{p}(\mathbb{N})$ such that

$$
\left\|z_{s}\right\|<\varepsilon \text { and }\left\|T^{s+1+k-b_{n}} z_{s}-e_{k}\right\|<\varepsilon .
$$

Fix $s \in S_{\eta, n}$, so that $s$ belongs to $\left[1, b_{m+1}-b_{m}\right)$ for some integer $m$ of $\mathcal{N}_{\eta, n}$ and

$$
\left|v_{m}\right| \prod_{i=b_{m+1}-s}^{b_{m+1}-1}\left|w_{i}\right|>\frac{1}{\eta}
$$

We define a vector $z_{s}$ by setting

$$
z_{s}:=\frac{1}{v_{m} \prod_{j=b_{n}+1}^{k} w_{j} \prod_{j=b_{m+1}-s}^{b_{m+1}-1} w_{j}} e_{b_{m+1}-s-1} .
$$

Then

$$
\left\|z_{s}\right\|=\frac{1}{\left|v_{m}\right| \prod_{j=b_{n}+1}^{k}\left|w_{j}\right| \prod_{j=b_{m+1}-s}^{b_{m+1}-1}\left|w_{j}\right|}<\frac{\eta}{\prod_{j=b_{n}+1}^{k}\left|w_{j}\right|}
$$

so that $\left\|z_{s}\right\|<\varepsilon$ for every $s \in S_{\eta, n}$ if $\eta$ is small enough. Moreover, since $\varphi(m)=n$ we also have

$$
T^{s+1+k-b_{n}} z_{s}=e_{k}-\frac{\prod_{j=b_{m}+1}^{b_{m}+k-b_{n}} w_{j}}{v_{m} \prod_{j=b_{m}+1}^{b_{m}+1} w_{j} \prod_{j=b_{n}+1}^{k} w_{j}} e_{b_{m}+k-b_{n}},
$$

and hence (since $m$ belongs to $\mathcal{N}_{\eta, n}$ )

$$
\left\|T^{s+1+k-b_{n}} z_{s}-e_{k}\right\|=\left|\frac{\prod_{j=b_{m}+1}^{b_{m}+k-b_{n}} w_{j}}{v_{m} \prod_{j=b_{m}+1}^{b_{m}+1} w_{j} \prod_{j=b_{n}+1}^{k} w_{j}}\right|<\frac{\prod_{j=b_{m}+1}^{b_{m}+k-b_{n}}\left|w_{j}\right|}{\prod_{j=b_{n}+1}^{k}\left|w_{j}\right|} \eta .
$$

Since the sequence of weights $\left(w_{j}\right)_{j \geq 1}$ is bounded by a positive constant $M$,

$$
\left\|T^{s+1+k-b_{n}} z_{s}-e_{k}\right\| \leq \frac{M^{b_{n+1}-b_{n}}}{\prod_{j=b_{n}+1}^{k}\left|w_{j}\right|} \eta
$$

and the quantity on the right hand side does not depend on $s \in S_{\eta, n}$. So we also have $\left\|T^{s+1+k-b_{n}} z_{s}-e_{k}\right\|<\varepsilon$ for every $s \in S_{\eta, n}$ provided $\eta$ is small enough.

For operators of $\mathrm{C}_{+}$-type, the statement of Proposition 6.14 can be slightly simplified.

Corollary 6.15. - Let $T=T_{v, w, \varphi, b}$ be an operator of $C_{+}$-type on $\ell_{p}(\mathbb{N})$. Suppose that for every $\varepsilon>0$, the set

$$
S_{\varepsilon}:=\bigcup_{k \in \mathcal{K}_{\varepsilon}}\left\{n \in \left[1, \Delta^{(k)}\left[;\left|v^{(k)}\right| \prod_{i=\Delta^{(k)}-n}^{\Delta^{(k)}-1}\left|w_{i}^{(k)}\right|>\frac{1}{\varepsilon}\right\} \quad\right.\right. \text { is cofinite, }
$$

where $\mathcal{K}_{\varepsilon}=\left\{k \geq 1:\left|v_{k}\right| \prod_{i=1}^{\Delta^{(k)}-1}\left|w_{i}^{(k)}\right|>\frac{1}{\varepsilon}\right\}$. Then $T$ is topologically mixing.

Proof. - This follows immediately from the special structure of $\mathrm{C}_{+}$-type operators and the definition of the map $\varphi$ in this case. 
We now use Fact 6.12 in order to formulate sufficient conditions for an operator of C-type to be not topologically mixing.

Proposition 6.16. - Let $T=T_{v, w, \varphi, b}$ be a C-type operator on $\ell_{p}(\mathbb{N})$. Let $\left(C_{n}\right)_{n \geq 0}$ be a sequence of positive numbers with $0<C_{n}<1$ for every $n \geq 0$. Assume that

$$
\left|v_{n}\right| \cdot \sup _{j \in\left[b_{\varphi(n)}, b_{\varphi(n)+1}\right)}\left(\prod_{s=b_{\varphi(n)}+1}^{j}\left|w_{s}\right|\right) \leq C_{n} \quad \text { for every } n \geq 0,
$$

and that there exists a positive constant $K$ such that for infinitely many integers $n \geq 0$, we have

$$
\sum_{l>n} C_{l}\left(b_{l+1}-b_{l}\right)^{1-\frac{1}{p}}\left(\sup _{b_{l+1}-2\left(b_{n+1}-b_{n}\right) \leq i<b_{l+1}} \prod_{s=i+1}^{b_{l+1}-1}\left|w_{s}\right|\right) \leq K .
$$

Then $T$ is not topologically mixing.

Proof. - Recall that we denote for each $l \geq 0$ by $P_{l}$ the canonical projection of $\ell_{p}(\mathbb{N})$ onto the finite-dimensional space $\operatorname{span}\left[e_{j} ; b_{l} \leq j<b_{l+1}\right]$. It is enough to show that if $n \geq 0$ satisfies the assumption (31), then

$$
\left\|P_{0} T^{2\left(b_{n+1}-b_{n}\right)} x\right\| \leq 1 \quad \text { for every } x \in B\left(0, \frac{1}{1+K}\right) .
$$

Indeed, this will imply that $2\left(b_{n+1}-b_{n}\right)$ does not belong to $\mathcal{N}_{T}\left(B\left(0, \frac{1}{1+K}\right), B\left(3 e_{0}, 1\right)\right)$ for every such $n$, and hence that $T$ is not topologically mixing.

Let us fix a vector $x \in B\left(0, \frac{1}{1+K}\right)$ and an integer $n \geq 0$ satisfying (31). Recalling that $T^{2\left(b_{n+1}-b_{n}\right)} e_{k}=e_{k}$ for every $0 \leq k<b_{n+1}$, we have

$$
P_{0} T^{2\left(b_{n+1}-b_{n}\right)} x=P_{0} \sum_{l=0}^{n} P_{l} x+P_{0} \sum_{l>n} T^{2\left(b_{n+1}-b_{n}\right)} P_{l} x=P_{0} x+\sum_{l>n} P_{0} T^{2\left(b_{n+1}-b_{n}\right)} P_{l} x .
$$

Moreover, if $l>n$, it follows from Fact 6.12 that

$$
\left\|P_{0} T^{2\left(b_{n+1}-b_{n}\right)} P_{l} x\right\| \leq C_{l}\left(b_{l+1}-b_{l}\right)^{1-\frac{1}{p}}\left(\sup _{b_{l+1}-2\left(b_{n+1}-b_{n}\right) \leq i<b_{l+1}} \prod_{s=i+1}^{b_{l+1}-1}\left|w_{s}\right|\right)\left\|P_{l} x\right\| .
$$

So we get that

$$
\left\|P_{0} T^{2\left(b_{n+1}-b_{n}\right)} x\right\| \leq\left\|P_{0} x\right\|+\sum_{l>n}\left\|P_{0} T^{2\left(b_{n+1}-b_{n}\right)} P_{l} x\right\| \leq\|x\|+K\|x\| \leq 1,
$$

which proves Proposition 6.16 .

After these technical preliminaries, we are now going to consider special classes of operators of $\mathrm{C}$-type, for which the general conditions for frequent hypercyclicity, $\mathcal{U}$-frequent hypercyclicity and topological mixing obtained previously become rather transparent. This will allow us to derive easily the promised counterexamples. 
6.5. Operators of $\mathbf{C}_{+, 1}$-type: $\mathbf{F H C}$ does not imply ergodic. - In this subsection, we restrict ourselves to operators of $\mathrm{C}_{+}$-type for which the parameters $v, w$, and $b$ satisfy the following conditions: for every $k \geq 1$,

$$
v^{(k)}=2^{-\tau^{(k)}} \quad \text { and } \quad w_{i}^{(k)}= \begin{cases}2 & \text { if } 1 \leq i \leq \delta^{(k)} \\ 1 & \text { if } \delta^{(k)}<i<\Delta^{(k)}\end{cases}
$$

where $\left(\tau^{(k)}\right)_{k \geq 1}$ and $\left(\delta^{(k)}\right)_{k \geq 1}$ are two strictly increasing sequences of integers with $\delta^{(k)}<$ $\Delta^{(k)}$ for every $k \geq 1$. We call such operators operators of $C_{+, 1}$-type.

If, in order to simplify matters, we assume that $\delta^{(k)}=2 \tau^{(k)}$ for every $k \geq 1$, then the key parameter of our counterexamples will be the quantity $\frac{\delta^{(k)}}{\Delta^{(k)}}$, i.e. the proportion of weights equal to 2 in each block $\left[b_{n}, b_{n+1}\right)$. For instance, if we consider the vector $x=2^{-\frac{\delta^{(k)}}{2}} e_{b_{2^{k-1}}}$, we remark that $T^{\Delta^{(k)}} x$ is close to $e_{0}$, and that the orbit of $x$ follows the orbit of $e_{0}$ during an interval of time proportional to $\delta^{(k)}$. Since the period of $x$ is equal to $2 \Delta^{(k)}$, it seems plausible in view of Theorem 5.31 that $T$ will be frequently hypercyclic as soon as $\lim \sup _{k \rightarrow \infty} \frac{\delta^{(k)}}{\Delta^{(k)}}>0$. On the other hand, we will show that if $\limsup _{k \rightarrow \infty} \frac{\delta^{(k)}}{\Delta^{(k)}}$ is too small, $T$ cannot be ergodic.

6.5.1. How to be FHC or UFHC. - Our first result gives a readable sufficient condition for an operator of $\mathrm{C}_{+, 1}$-type to be chaotic and frequently hypercyclic.

Theorem 6.17. - Let $T=T_{v, w, \varphi, b}$ be an operator of $C_{+, 1}$-type on $\ell_{p}(\mathbb{N})$.

(1) If $\limsup _{k \rightarrow \infty}\left(\delta^{(k)}-\tau^{(k)}\right)=\infty$, then $T$ is chaotic.

(2) If $\limsup _{k \rightarrow \infty} \frac{\delta^{(k)}-\tau^{(k)}}{\Delta^{(k)}}>0$, then $T$ is chaotic and frequently hypercyclic.

Proof. - The first statement is a direct consequence of Proposition 6.5. for every $n \geq 0$, the expressions of $v^{(k)}$ and $\left(w_{j}^{(k)}\right)_{1 \leq j<\Delta^{(k)}}$ combined with the fact that $\varphi\left(\left[2^{k}, 2^{k+1}\right)\right)=$ $\left[0,2^{k-1}\right)$ for every $k \geq 1$ yield that

$$
\limsup _{\substack{N \rightarrow \infty \\ N \in \varphi^{-1}(n)}}\left|v_{N}\right| \cdot \prod_{j=b_{N}+1}^{b_{N+1}-1}\left|w_{j}\right|=\limsup _{k \rightarrow \infty} 2^{\delta^{(k)}-\tau^{(k)}}=\infty .
$$

As to the second statement, it is a consequence of Theorem 6.9. Let us fix a real number $\alpha$ with

$$
0<\alpha<\frac{1}{2} \limsup _{k \rightarrow \infty} \frac{\delta^{(k)}-\tau^{(k)}}{\Delta^{(k)}} .
$$

Fix also $C \geq 1$ and $k_{0} \geq 1$. By our assumption and the fact that $\Delta^{(k)}$ tends to infinity as $k$ tends to infinity, there exists an integer $k \geq k_{0}$ such that

$$
\frac{\delta^{(k)}-\tau^{(k)}}{\Delta^{(k)}}>2 \alpha \text { and } \alpha \Delta^{(k)}>\log _{2} C .
$$

We apply Theorem 6.9 with $m=\Delta^{(k)}-1$. We have

$$
\left|v^{(k)}\right| \cdot \prod_{i=1}^{\Delta^{(k)}-1}\left|w_{i}^{(k)}\right|=2^{\delta^{(k)}-\tau^{(k)}}>2^{2 \alpha \Delta^{(k)}} \geq C,
$$


which gives condition (25) of Theorem 6.9, As for condition (26), we have for every $0 \leq m^{\prime} \leq \alpha \Delta^{(k)}$ :

$$
\left|v^{(k)}\right| \cdot \prod_{i=m^{\prime}+1}^{\Delta^{(k)}-1}\left|w_{i}^{(k)}\right| \geq 2^{\delta^{(k)}-m^{\prime}-\tau^{(k)}} \geq 2^{\delta^{(k)}-\alpha \Delta^{(k)}-\tau^{(k)}}>C .
$$

It thus follows from Theorem 6.9 that $T$ is frequently hypercyclic.

The next result gives, under some additional assumptions, necessary and sufficient conditions for an operator of $\mathrm{C}_{+, 1}$-type to be $\mathcal{U}$-frequently hypercyclic or frequently hypercyclic (which turn out to be same under the additional assumptions).

Theorem 6.18. - Let $T=T_{v, w, \varphi, b}$ be an operator of $C_{+, 1}$-type on $\ell_{p}(\mathbb{N})$. We additionally assume that the sequence $\left(\gamma_{k}\right)_{k \geq 1}$ defined by $\gamma_{k}:=2^{\delta^{(k-1)}-\tau^{(k)}}\left(\Delta^{(k)}\right)^{1-\frac{1}{p}}$ for every $k \geq 1$ is a non-increasing sequence, and that the following two conditions hold true:

$$
\limsup _{k \rightarrow \infty} \frac{\tau^{(k)}}{\delta^{(k)}}<1 \quad \text { and } \quad \sum_{k \geq 1} 2^{k} \gamma_{k}^{1 / 2} \leq 1 .
$$

Then the following assertions are equivalent:

(1) $T$ is $\mathcal{U}$-frequently hypercyclic;

(2) $T$ is frequently hypercyclic;

(3) $\limsup \delta^{(k)} / \Delta^{(k)}>0$.

Proof. - Since $\limsup _{k \rightarrow \infty} \frac{\tau^{(k)}}{\delta^{(k)}}<1$, Condition (3) is equivalent to $\limsup _{k \rightarrow \infty} \frac{\delta^{(k)}-\tau^{(k)}}{\Delta^{(k)}}>0$. It follows from this observation and Theorem6.17 that (3) implies (2), which of course implies (1). It remains to prove that if $\limsup _{k \rightarrow \infty} \frac{\delta^{(k)}}{\Delta^{(k)}}=0, T$ is not $\mathcal{U}$-frequently hypercyclic. Given a hypercyclic vector $x$ for $T$, we need to show that $x$ cannot be a $\mathcal{U}$-frequently hypercyclic vector for $T$, and for this it suffices to find $C,\left(\beta_{l}\right)_{l \geq 1},\left(X_{l}\right)_{l \geq 1}$, and $\left(N_{l}\right)_{l \geq 1}$ satisfying assumptions (1), (2), (3) and (C) of Theorem 6.10. This is done thanks to Facts 6.12 and 6.13. We first fix the sequence $\left(\beta_{l}\right)_{l \geq 1}$ by setting

$$
\beta_{l}=4 \gamma_{k} \quad \text { for every } l \in\left[2^{k-1}, 2^{k}\right), k \geq 1 .
$$

Since the sequence $\left(\gamma_{k}\right)_{k \geq 1}$ is non-increasing, the sequence $\left(\beta_{l}\right)_{l \geq 1}$ is non-increasing. Also

$$
\sum_{l \geq 1} \sqrt{\beta_{l}}=\sum_{k \geq 1} 2^{k-1}\left(4 \gamma_{k}\right)^{1 / 2} \leq 1
$$

by assumption. We have for every $k \geq 1$ and every $n \in\left[2^{k-1}, 2^{k}\right)$

$$
\left|v_{n}\right| \cdot \sup _{j \in\left[b_{\varphi(n)}, b_{\varphi(n)+1}\right)} \prod_{s=b_{\varphi(n)+1}^{j}}^{j}\left|w_{s}\right| \leq 2^{\delta^{(k-1)}-\tau^{(k)}} .
$$


If we set $C_{n}=2^{\delta^{(k-1)}-\tau^{(k)}}$ for every $n \in\left[2^{k-1}, 2^{k}\right)$, Fact 6.12 implies that for every $k \geq 0$, every $l \in\left[2^{k-1}, 2^{k}\right)$ and every $0 \leq n<l$,

$$
\begin{aligned}
\sup _{j \geq 0}\left\|P_{n} T^{j} P_{l} x\right\| & \leq 2^{\delta^{(k-1)}-\tau^{(k)}} \cdot\left(\Delta^{(k)}\right)^{1-\frac{1}{p}} \cdot\left\|\sum_{i=b_{l}}^{b_{l+1}-1}\left(\prod_{s=i+1}^{b_{l+1}-1} w_{s}\right) x_{i} e_{i}\right\| \\
& \leq \frac{\beta_{l}}{4} \cdot\left\|\sum_{i=b_{l}}^{b_{l+1}-1}\left(\prod_{s=i+1}^{b_{l+1}-1} w_{s}\right) x_{i} e_{i}\right\|
\end{aligned}
$$

and, for every $1 \leq N \leq \Delta^{(k)}$,

$$
\sup _{0 \leq j \leq N}\left\|P_{n} T^{j} P_{l} x\right\| \leq \frac{\beta_{l}}{4} \cdot\left(\prod_{i=\Delta^{(k)}-N+1}^{\Delta^{(k)}-1}\left|w_{i}^{(k)}\right|\right) \cdot\left\|P_{l} x\right\| .
$$

We now set $N_{l}:=\Delta^{(k)}-\delta^{(k)}$ for every $l \in\left[2^{k-1}, 2^{k}\right)$ and every $k \geq 1$. Remembering that $w_{i}^{(k)}=1$ if $\delta^{k}<i<\Delta^{(k)}$, we obtain that

$$
\sup _{0 \leq j \leq N_{l}}\left\|P_{n} T^{j} P_{l} x\right\| \leq \frac{\beta_{l}}{4}\left\|P_{l} x\right\| \quad \text { for every } l \geq 1 .
$$

Finally, we set for every $k \geq 1$ and every $l \in\left[2^{k-1}, 2^{k}\right)$

$$
X_{l}:=\left\|\sum_{i=b_{l}}^{b_{l+1}-1}\left(\prod_{s=i+1}^{b_{l+1}-1} w_{s}\right) x_{i} e_{i}\right\| .
$$

Obviously, $\left\|P_{l} x\right\| \leq X_{l}$, and we have proved that for every $0 \leq n<l$,

$$
\sup _{j \geq 0}\left\|P_{n} T^{j} P_{l} x\right\| \leq \frac{\beta_{l}}{4} X_{l} \text { and } \sup _{0 \leq j \leq N_{l}}\left\|P_{n} T^{j} P_{l} x\right\| \leq \frac{\beta_{l}}{4}\left\|P_{l} x\right\| .
$$

It remains to check that condition $(\mathrm{C})$ of Theorem 6.10 holds true, and for this we use Fact 6.13.

We have $w_{i}^{(k)}=1$ for every $i \in\left(\delta^{(k)}, \Delta^{(k)}\right)$, and $\prod_{i=\delta^{(k)}+1}^{\Delta^{(k)}-1} w_{i}^{(k)}=1$. The assumptions of Fact 6.13 are thus satisfied for $k_{0}=\delta^{(k)}$ and $k_{1}=\Delta^{(k)}$ if $l \in\left[2^{k-1}, 2^{k}\right)$, and we get

$$
\frac{1}{J+1} \#\left\{0 \leq j \leq J ;\left\|P_{l} T^{j} P_{l} x\right\| \geq X_{l} / 2\right\} \geq 1-2 \delta^{(k)}\left(\frac{1}{J+1}+\frac{1}{\Delta^{(k)}}\right)
$$

for every $J \geq 0$, every $k \geq 1$, and every $l \in\left[2^{k-1}, 2^{k}\right)$. Therefore,

$$
\inf _{J \geq N_{l}} \frac{1}{J+1} \#\left\{0 \leq j \leq J ;\left\|P_{l} T^{j} P_{l} x\right\| \geq X_{l} / 2\right\} \geq 1-2 \delta^{(k)}\left(\frac{1}{\Delta^{(k)}-\delta^{(k)}+1}+\frac{1}{\Delta^{(k)}}\right)
$$

for every $k \geq 1$ and every $l \in\left[2^{k-1}, 2^{k}\right)$, and it follows that

$$
\begin{aligned}
\liminf _{l \rightarrow \infty} \inf _{J \geq N_{l}} \frac{1}{J+1} & \#\left\{0 \leq j \leq J ;\left\|P_{l} T^{j} P_{l} x\right\| \geq X_{l} / 2\right\} \\
& \geq \liminf _{k \rightarrow \infty}\left(1-2 \delta^{(k)}\left(\frac{1}{\Delta^{(k)}-\delta^{(k)}+1}+\frac{1}{\Delta^{(k)}}\right)\right)=1,
\end{aligned}
$$

since $\limsup _{k \rightarrow \infty} \frac{\delta^{(k)}}{\Delta^{(k)}}=0$ by assumption. Theorem 6.10 eventually yields that $T$ is not $\mathcal{U}$-frequently hypercyclic, and this concludes the proof. 
Remark 6.19. - Theorem 6.18 implies the main result of [39], i.e. that there exist chaotic operators on $\ell_{p}(\mathbb{N})$ which are not $\mathcal{U}$-frequently hypercyclic.

6.5.2. A word about the OSP. - In this short subsection, we show that there exist $\mathrm{C}_{+, 1^{-}}$ type operators on $\ell_{2}(\mathbb{N})$ which satisfy the assumptions of the criterion for frequent hypercyclicity stated in Theorem 5.31, and yet do not have the Operator Specification Property (recall that we have proved in Section 5.4.1 that operators with the OSP do satisfy the assumptions of Theorem 5.31). The following corollary is a simple consequence of Proposition 6.16 applied to $\mathrm{C}_{+, 1}$-type operators.

Corollary 6.20. - Let $T=T_{v, w, \varphi, b}$ be an operator of $C_{+, 1}$-type on $\ell_{p}(\mathbb{N})$ and let $\gamma_{k}=$ $2^{\delta^{(k-1)}-\tau^{(k)}}\left(\Delta^{(k)}\right)^{1-\frac{1}{p}}$ for every $k \geq 1$. Assume that $\tau^{(k)}>\delta^{(k-1)}$ for every $k \geq 1$ and that there exists a positive constant $K$ such that, for every $k_{0} \geq 1$,

$$
\sum_{k>k_{0}} 2^{k} \gamma_{k} \leq K 4^{-\Delta^{\left(k_{0}\right)}}
$$

Then $T$ is not topologically mixing.

Proof. - Let $n \geq 1$ be an integer, and let $k_{n}$ be the unique integer such that $2^{k_{n}-1} \leq n<$ $2^{k_{n}}$. Then $v_{n}=v^{\left(k_{n}\right)}=2^{-\tau^{\left(k_{n}\right)}}$ and $\varphi(n)<2^{k_{n}-1}$, and setting

$$
C_{n}:=\left|v_{n}\right| \sup _{j \in\left[b_{\varphi(n)}, b_{\varphi(n)+1}\right)}\left(\prod_{s=b_{\varphi(n)}+1}^{j}\left|w_{s}\right|\right),
$$

we have $0<C_{n} \leq 2^{-\tau^{\left(k_{n}\right)}} 2^{\delta^{\left(k_{n}-1\right)}}<1$. Moreover, if $n$ is an integer of the form $n=2^{k}-1$ for some integer $k \geq 1$, then $b_{n+1}-b_{n}=\Delta^{(k)}$, and moreover $k_{l}>k$ for every $l>n$. It follows that

$$
\sum_{l>n} C_{l}\left(b_{l+1}-b_{l}\right)^{1-\frac{1}{p}}\left(\sup _{b_{l+1}-2\left(b_{n+1}-b_{n}\right) \leq i<b_{l+1}} \prod_{s=i+1}^{b_{l+1}-1}\left|w_{s}\right|\right)
$$

is less than

$$
\leq \sum_{r>k} 2^{r-1} 2^{\delta^{(r-1)}-\tau^{(r)}}\left(\Delta^{(r)}\right)^{1-\frac{1}{p}} \cdot 2^{2 \Delta^{(k)}} \leq K .
$$

Hence Proposition 6.16 applies, and $T$ is not topologically mixing.

Since the condition appearing in Corollary 6.20 is compatible with those of Theorem 6.18 above (see Example 6.23), and since operators with the OSP are topologically mixing, we immediately deduce:

Corollary 6.21. - There exist operators on $\ell_{p}(\mathbb{N})$ which satisfy the assumptions of Theorem 6.18 and yet do not have the Operator Specification Property.

Since the assumptions of Theorem 6.18 imply those of Theorem 5.31, it follows that there exist operators on $\ell_{p}(\mathbb{N})$ which satisfy the criterion for frequent hypercyclicity stated in Theorem 5.31 without having the OSP. In particular, this criterion for frequent hypercyclicity is strictly stronger than the "classical" one. 
6.5.3. FHC but not ergodic. - We are now ready to give examples of operators of $\mathrm{C}_{+, 1^{-}}$ type which are frequently hypercyclic but not ergodic.

Theorem 6.22. - Let $T$ be an operator of $C_{+, 1}$-type on $\ell_{p}(\mathbb{N})$, and set, for every $k \geq 1$, $\gamma_{k}:=2^{\delta^{(k-1)}-\tau^{(k)}}\left(\Delta^{(k)}\right)^{1-\frac{1}{p}}$. Suppose that the sequence $\left(\gamma_{k}\right)_{k \geq 1}$ is non-increasing, and that the following three conditions hold true:

$$
\sum_{k \geq 1} 2^{k} \gamma_{k}^{1 / 2} \leq 1, \quad \limsup _{k \rightarrow \infty} \frac{\tau^{(k)}}{\delta^{(k)}}<1 \quad \text { and } \quad 0<\limsup _{k \rightarrow \infty} \frac{\delta^{(k)}}{\Delta^{(k)}} \leq \frac{1}{5} .
$$

Then $T$ is frequently hypercyclic but $c(T)<1$, so that $T$ is not ergodic.

Proof. - The frequent hypercyclicity of $T$ follows from Theorem 6.18, Let us show that $c(T)<1$. Recall that $c(T)$ is characterized by the following property (see [29, Rem. 4.6]): for quasi-all hypercyclic vectors $x$ for $T$ in the Baire category sense, we have

$$
c(T)=\overline{\operatorname{dens}}(x, B(0, \varepsilon)) \quad \text { for every } \varepsilon>0 .
$$

Let us fix a hypercyclic vector $x$ for $T$ such that (32) holds true. By Lemma 6.11 combined with the end of the proof of Theorem 6.18, we know that there exists an $\varepsilon>0$ such that

$$
\left.\underline{\text { dens }} \mathcal{N}_{T}\left(x, B(0, \varepsilon)^{c}\right)\right) \geq \liminf _{l \rightarrow \infty} \inf _{J \geq N_{l}} \frac{1}{J+1} \#\left\{0 \leq j \leq J ;\left\|P_{l} T^{j} P_{l} x\right\| \geq X_{l} / 2\right\}
$$

(recall that we proved above that under the assumption (3) of Theorem 6.18, the assumptions of Theorem 6.10 are satisfied with $C=1 / 4$, so that $2 C X_{l}=X_{l} / 2$ ). Using an inequality obtained at the end of the proof of Theorem 6.18, we get

$$
\underline{\text { dens }} \mathcal{N}_{T}\left(x, B(0, \varepsilon)^{c}\right) \geq \liminf _{k \rightarrow \infty}\left(1-2 \delta^{(k)}\left(\frac{1}{\Delta^{(k)}-\delta^{(k)}+1}+\frac{1}{\Delta^{(k)}}\right)\right)
$$

so that

$$
\begin{aligned}
\overline{\operatorname{dens}} \mathcal{N}_{T}(x, B(0, \varepsilon)) & \leq \limsup _{k \rightarrow \infty}\left(\frac{2 \delta^{(k)}}{\Delta^{(k)}-\delta^{(k)}+1}+\frac{2 \delta^{(k)}}{\Delta^{(k)}}\right) \\
& =\limsup _{k \rightarrow \infty}\left(\frac{2 \delta^{(k)}}{\Delta^{(k)}} \cdot \frac{1}{1-\frac{\delta^{(k)}}{\Delta^{(k)}}+\frac{1}{\Delta^{(k)}}}+\frac{2 \delta^{(k)}}{\Delta^{(k)}}\right) .
\end{aligned}
$$

It follows that

$$
c(T) \leq \limsup _{k \rightarrow \infty}\left(\frac{2 \delta^{(k)}}{\Delta^{(k)}} \cdot \frac{1}{1-\frac{\delta^{(k)}}{\Delta^{(k)}}+\frac{1}{\Delta^{(k)}}}+\frac{2 \delta^{(k)}}{\Delta^{(k)}}\right) .
$$

Our assumption thus implies that

$$
c(T) \leq \frac{2 / 5}{1-1 / 5}+\frac{2}{5}=\frac{1}{2}+\frac{2}{5}<1,
$$

which proves that $T$ is not ergodic.

Example 6.23. - Let $C$ be a positive integer, and consider the operator of $\mathrm{C}_{+, 1}$-type $T$ on $\ell_{p}(\mathbb{N})$ associated to the parameters

$$
\tau^{(k)}=2^{C k}, \quad \delta^{(k)}=2 \cdot 2^{C k} \quad \text { and } \quad \Delta^{(k)}=10 \cdot 2^{C k}, \quad k \geq 1 .
$$


If $C$ is sufficiently large, $T$ is frequently hypercyclic but not ergodic. Besides, it is also not topologically mixing.

Proof. - With this choice of parameters, and assuming that $C \geq 2$, we have

$$
\gamma_{k}=2^{\left(2 \cdot 2^{C(k-1)}-2^{C k}\right)} \cdot 10^{1-\frac{1}{p}} \cdot 2^{C k\left(1-\frac{1}{p}\right)} \leq 10 \cdot 2^{-\frac{1}{2} 2^{C k}} \cdot 2^{C k}
$$

for every $k \geq 1$. So it is not hard to check that if $C$ is sufficiently large, the sequence $\left(\gamma_{k}\right)_{k \geq 1}$ is non-increasing and satisfies $\sum_{k>1} 2^{k} \gamma_{k}^{1 / 2} \leq 1$. The other assumptions of Theorem 6.22 are clearly satisfied, and hence $T$ is frequently hypercyclic but not ergodic.

In order to show that $T$ is also not topologically mixing if $C$ is large enough, we use Proposition 6.16. If $n$ is any integer of the form $n=2^{k_{0}}-1$, where $k_{0} \geq 1$ is any integer, then

$$
\begin{aligned}
\sum_{l>n} C_{l}\left(b_{l+1}-b_{l}\right)^{1-\frac{1}{p}}\left(\sup _{b_{l+1}-2\left(b_{n+1}-b_{n}\right) \leq k<b_{l+1}} \prod_{s=k+1}^{b_{l+1}-1}\left|w_{s}\right|\right) & =\sum_{k>k_{0}} 2^{k-1} 2^{\delta^{(k-1)}-\tau^{(k)}}\left(\Delta^{(k)}\right)^{1-\frac{1}{p}} \\
& =\sum_{k>k_{0}} 2^{k-1} \gamma_{k}
\end{aligned}
$$

If $C$ is sufficiently large, then $\sum_{k=1}^{\infty} 2^{k-1} \gamma_{k}<1$; so we deduce from Proposition 6.16 (by considering $K:=1$ and $n:=2^{k_{0}}-1$ for every $k_{0} \geq 1$ ) that $T$ is not topologically mixing. Notice that $T$ also satisfied the assumptions of Corollary 6.20.

Since the conditions of Theorem 6.18 make no difference between $\mathcal{U}$-frequent and frequent hypercyclicity, we will need to introduce another family of operators of $\mathrm{C}_{+}$-type in order to construct examples of $\mathcal{U}$-frequent hypercyclic operators on $\ell_{p}(\mathbb{N})$ which are not frequently hypercyclic. But before moving over to the presentation of this new class of operators, we give in the next subsection a corollary of Theorem 6.22 concerning infinite direct sums of frequently hypercyclic operators.

6.6. Infinite direct sums of frequently hypercyclic operators. - A well-known open question, dating back to [5], asks whether the direct sum $T \oplus T$ of a frequently hypercyclic operator $T$ with itself has to be frequently hypercyclic. (This is the analogue of Herreros " $T \oplus T$ problem" for frequent hypercyclicity.) By [32], $T \oplus T$ is hypercyclic as soon as $T$ is $\mathcal{U}$-frequently hypercyclic. This result of $\mathbf{3 2}$ also implies that the direct sum of two (and hence, of infinitely many) $\mathcal{U}$-frequently hypercyclic operators is hypercyclic. Indeed, let $T_{1}$ and $T_{2}$ be two bounded operators acting respectively on the Banach spaces $X_{1}$ and $X_{2}$, and let $U_{1}, V_{1}$ and $U_{2}, V_{2}$ be non-empty open subsets of $X_{1}$ and $X_{2}$ respectively. By [32], the set $\mathcal{N}_{T_{1}}\left(U_{1}, V_{1}\right)$ has bounded gaps. Also, since $T_{2}$ is topologically weakly mixing, the set $\mathcal{N}_{T_{2}}\left(U_{2}, V_{2}\right)$ contains arbitrarily long intervals. It follows that $T_{1} \oplus T_{2}$ is hypercyclic.

Apart from this result of [32, nothing seems to be known concerning this question of [5]. More generally, it seems to be unknown whether the direct sum $T_{1} \oplus T_{2}$ of two frequently hypercyclic operators is necessarily frequently hypercyclic, or even $\mathcal{U}$-frequently hypercyclic. To the best of our knowledge, this question is open even for infinite direct sums of frequently hypercyclic operators. Our aim in this subsection is to use operators of $C_{+1}$-type on $\ell_{p}(\mathbb{N})$ to prove the following result. 
Theorem 6.24. - Let $p>1$. There exists a sequence $\left(T_{n}\right)_{n \geq 1}$ of frequently hypercyclic operators on $\ell_{p}(\mathbb{N})$ such that the $\ell_{p}$-sum operator $T=\bigoplus_{n \geq 1} T_{n}$ acting on $X=\bigoplus_{n \geq 1} \ell_{p}(\mathbb{N})$ is not $\mathcal{U}$-frequently hypercyclic.

The proof of Theorem 6.24 relies on Theorem 6.22, combined with an elementary lemma providing an upper bound for the parameter $c(T)$ of a direct sum operator:

Lemma 6.25. - Let $T_{1}$ and $T_{2}$ be two bounded operators acting respectively on the Banach spaces $X_{1}$ and $X_{2}$. Fix $p>1$, and let $T=T_{1} \oplus_{\ell_{p}} T_{2}$ be the $\ell_{p}$-sum operator of $T_{1}$ and $T_{2}$, acting on $X=X_{1} \oplus_{\ell_{p}} X_{2}$. Moreover, assume that $T$ is hypercyclic. Then $c(T) \leq \min \left(c_{1}(T), c_{2}(T)\right)$.

Proof of Lemma 6.25. - Let $c \in[0,1]$ be such that $\overline{\operatorname{dens}} \mathcal{N}_{T}\left(x, B_{X}(0,1)\right) \geq c$ for a comeager set of vectors $x$ of $X$. For any such vector $x=x_{1} \oplus x_{2}$, with $x_{1} \in X_{1}$ and $x_{2} \in X_{2}$, there exists a subset $D_{x}$ of $\mathbb{N}$ with $\overline{\text { dens }} D_{x} \geq c$ such that $\left(\left\|T_{1}^{n} x_{1}\right\|^{p}+\left\|T_{2}^{n} x_{2}\right\|^{p}\right)^{1 / p}<1$ for every $n \in D_{x}$. Thus $\overline{\text { dens }} \mathcal{N}_{T_{1}}\left(x_{1}, B_{X_{1}}(0,1)\right) \geq c$ for a comeager subset of vectors $x_{1} \in X_{1}$, so that $c\left(T_{1}\right) \geq c$; and likewise, $c\left(T_{2}\right) \geq c$. Hence $\min \left(c_{1}(T), c_{2}(T)\right) \geq c$ for any $c$ as above, which proves the lemma.

As an easy consequence of Lemma 6.25, we obtain

Lemma 6.26. - For each $n \geq 1$, let $T_{n}$ be a bounded operator on a Banach space $X_{n}$. Fix $p>1$, and let $T=\bigoplus_{\ell_{p}} T_{n}$ be the $\ell_{p}$-sum of the operators $T_{n}, n \geq 1$, acting on the space $X=\bigoplus_{\ell_{p}} X_{n}$. Moreover, assume that $T$ is hypercyclic. Then

$$
c(T)=\inf _{n \geq 1} c\left(T_{1} \oplus \cdots \oplus T_{n}\right) \leq \inf _{n \geq 1} c\left(T_{n}\right) .
$$

Proof of Lemma 6.26. - It follows directly from Lemma 6.25 that

$$
c(T) \leq \inf _{n \geq 1} c\left(T_{1} \oplus \cdots \oplus T_{n}\right) \leq \inf _{n \geq 1} c\left(T_{n}\right),
$$

so we only have to prove that

$$
c(T) \geq \inf _{n \geq 1} c\left(T_{1} \oplus \cdots \oplus T_{n}\right) .
$$

If the infimum $\inf _{n \geq 1} c\left(T_{1} \oplus \cdots \oplus T_{n}\right)$ is equal to 0 , there is nothing to prove. Suppose that $\inf _{n \geq 1} c\left(T_{1} \oplus \cdots \oplus T_{n}\right)>0$ and consider a number $c$ such that $0 \leq c<\inf _{n \geq 1} c\left(T_{1} \oplus \cdots \oplus T_{n}\right)$. Let $U$ be a non-empty open subset of $X$. There exist an integer $n \geq 1$ and, for every $1 \leq$ $i \leq n$, a non-empty open subset $U_{i}$ of $X_{i}$, such that $U$ contains the set $U_{1} \oplus \cdots \oplus U_{n} \oplus 0 \oplus \cdots$. Since $c<\inf _{n \geq 1} c\left(T_{1} \oplus \cdots \oplus T_{n}\right)$, there exist a vector $x=x_{1} \oplus \cdots \oplus x_{n} \in U_{1} \oplus \cdots \oplus U_{n}$ and a subset $D$ of $\mathbb{N}$ with $\overline{\operatorname{dens}} D \geq c$ such that $\left(\sum_{i=1}^{n}\left\|T_{i}^{k} x_{i}\right\|^{p}\right)^{1 / p}<1$ for every $k \in D$. Setting $x=x_{1} \oplus \cdots \oplus x_{n} \oplus 0 \oplus \cdots$, we deduce that $x$ is a vector of $U$ which satisfies $\overline{\operatorname{dens}} \mathcal{N}_{T}\left(x, B_{X}(0,1)\right) \geq c$. The set of vectors $x \in X$ such that $\overline{\operatorname{dens}} \mathcal{N}_{T}\left(x, B_{X}(0,1)\right) \geq c$ is thus dense in $X$, and the usual argument shows that this set is in fact comeager in $X$. It follows that $c(T) \geq c$, from which we deduce that $c(T) \geq \inf _{n \geq 1} c\left(T_{1} \oplus \cdots \oplus T_{n}\right)$. This concludes the proof of Lemma 6.25.

Proof of Theorem 6.24. - Let $\left(T_{n}\right)_{n \geq 1}$ be a sequence of operators satisfying the assumptions of Theorem 6.22. Denoting for each $n \geq 1$ by $\left(\tau_{n}^{(k)}\right)_{k \geq 1},\left(\delta_{n}^{(k)}\right)_{k \geq 1}$, and $\left(\Delta_{n}^{(k)}\right)_{k \geq 1}$ the sequences of parameters associated to the operator $T_{n}$, we suppose that

$$
0<\limsup _{k \rightarrow \infty} \frac{\delta_{n}^{(k)}}{\Delta_{n}^{(k)}} \leq 2^{-n}
$$


for every $n \geq 1$. By Theorem 6.22, all the operators $T_{n}$ are frequently hypercyclic. On the other hand, it follows from (33) that

$$
\begin{aligned}
c\left(T_{n}\right) & \leq \limsup _{k \rightarrow \infty}\left(2 \frac{\delta_{n}^{(k)}}{\Delta_{n}^{(k)}} \cdot \frac{1}{1-\frac{\delta_{n}^{(k)}}{\Delta_{n}^{(k)}}+\frac{1}{\Delta_{n}^{(k)}}}+2 \frac{\delta_{n}^{(k)}}{\Delta_{n}^{(k)}}\right) \\
& \leq 2 \cdot 2^{-n} \cdot \frac{1}{1-2^{-n}}+2.2^{-n} \leq 6 \cdot 2^{-n} \quad \text { for every } n \geq 1,
\end{aligned}
$$

so that $c\left(T_{n}\right)$ tends to 0 as $n$ tends to infinity. By Lemma 6.26, the operator $T=\bigoplus_{\ell_{p}} T_{n}$ (which is hypercyclic) satisfies $c(T)=0$. Hence $T$ is not $\mathcal{U}$-frequently hypercyclic.

6.7. Operators of $\mathrm{C}_{+, 2}$-type: UFHC does not imply FHC. - In this subsection, we impose the following restrictions on the parameters $v, w$, and $b$ of a $\mathrm{C}_{+}$-type operator $T_{v, w, \varphi, b}:$ for every $k \geq 1$,

$$
v^{(k)}=2^{-\tau^{(k)}} \text { and } \quad w_{i}^{(k)}= \begin{cases}2 & \text { if } 1 \leq i \leq \delta^{(k)} \\ 1 & \text { if } \delta^{(k)}<i<\Delta^{(k)}-3 \delta^{(k)} \\ 1 / 2 & \text { if } \Delta^{(k)}-3 \delta^{(k)} \leq i<\Delta^{(k)}-2 \delta^{(k)} \\ 2 & \text { if } \Delta^{(k)}-2 \delta^{(k)} \leq i<\Delta^{(k)}-\delta^{(k)} \\ 1 & \text { if } \Delta^{(k)}-\delta^{(k)} \leq i<\Delta^{(k)}\end{cases}
$$

where $\left(\tau^{(k)}\right)_{k \geq 1}$ and $\left(\delta^{(k)}\right)_{k \geq 1}$ are two strictly increasing sequences of integers, satisfying $4 \delta^{k}<\Delta^{(k)}$ for every $k \geq 1$. We call operators satisfying these conditions operators of $C_{+, 2}$-type. Observe that we still have with this definition

$$
\prod_{i=1}^{\Delta^{(k)}-1} w_{i}^{(k)}=2^{\delta^{(k)}} \quad \text { for every } k \geq 1 .
$$

This choice of weights is motivated by the differences between the assumptions of Theorem 5.14 and those of Theorem 5.31. If we assume for simplicity that $\delta^{(k)}=2 \tau^{(k)}$ for every $k \geq 1$ and if we consider the vector $x:=2^{-\frac{\delta^{(k)}}{2}} e_{b_{2^{k-1}+\Delta^{(k)}}-2 \delta^{(k)}}$, we observe that $T^{2 \delta^{(k)}} x$ is close to $e_{0}$ and that the orbit of $x$ follows the orbit of $e_{0}$ during an interval of time proportional to $\delta^{(k)}$. In view of Theorem [5.14, it thus seems likely that $T$ will be $\mathcal{U}$-frequently hypercyclic. However, if $\lim _{k \rightarrow \infty} \frac{\delta^{(k)}}{\Delta^{(k)}}=0$, it also seems plausible that $T$ will not satisfy the assumptions of Theorem 5.31, and hence will possibly not be frequently hypercyclic.

6.7.1. How to be FHC or UFHC. - In this subsection, we present some sufficient conditions for an operator of $\mathrm{C}_{+, 2}$-type to be $\mathcal{U}$-frequently hypercyclic.

Theorem 6.27. - Let $T=T_{v, w, \varphi, b}$ be an operator of $C_{+, 2}$-type on $\ell_{p}(\mathbb{N})$.

(1) If $\limsup _{k \rightarrow \infty}\left(\delta^{(k)}-\tau^{(k)}\right)=\infty$, then $T$ is chaotic.

(2) If $\limsup _{k \rightarrow \infty} \frac{\tau^{(k)}}{\delta^{(k)}}<1$, then $T$ is chaotic and $\mathcal{U}$-frequently hypercyclic.

(3) If $\limsup _{k \rightarrow \infty} \frac{\delta^{(k)}-\tau^{(k)}}{\Delta^{(k)}}>0$, then $T$ is chaotic and frequently hypercyclic. 
Proof. - The proofs of assertions (1) and (3) are completely similar to the ones given in the proof of Theorem 6.17. As for (2), it is a consequence of Theorem 6.7. Let us fix

$$
0<\alpha<\frac{1}{3}-\frac{1}{3} \limsup _{k \rightarrow \infty} \frac{\tau^{(k)}}{\delta^{(k)}} .
$$

Fix also $C \geq 1$, and an integer $k_{0} \geq 1$. There exists an integer $k \geq k_{0}$ such that

$$
\frac{\tau^{(k)}}{\delta^{(k)}}<1-3 \alpha \text { and } \alpha \delta^{(k)}>\log _{2} C .
$$

We then have

$$
\left|v^{(k)}\right| \cdot \prod_{i=\Delta^{(k)}-2 \delta^{(k)}}^{\Delta^{(k)}-1}\left|w_{i}^{(k)}\right|=2^{\delta^{(k)}-\tau^{(k)}}>2^{3 \alpha \delta^{(k)}}>C
$$

and, for every $0 \leq m^{\prime} \leq 2 \alpha \delta^{(k)}$ :

$$
\left|v^{(k)}\right| \cdot \prod_{i=m^{\prime}+1}^{\Delta^{(k)}-1}\left|w_{i}^{(k)}\right| \geq 2^{(1-2 \alpha) \delta^{(k)}-\tau^{(k)}}>2^{\alpha \delta^{(k)}}>C
$$

Applying Theorem 6.7 with $m=2 \delta^{(k)}$, it follows that $T$ is $\mathcal{U}$-frequently hypercyclic.

We see from this proof that the new structure of the weights $\left(w_{j}^{(k)}\right)_{1 \leq j<\Delta^{(k)}}, k \geq 1$, compared with the case of operators of $\mathrm{C}_{+, 1}$-type, allows us to provide different conditions for $\mathcal{U}$-frequent hypercyclicity and frequent hypercyclicity of operators of $\mathrm{C}_{+, 2^{-}}$-type. Our next result gives, under some additional assumptions, a necessary and sufficient condition for frequent hypercyclicity of operators of $\mathrm{C}_{+, 2}$-type which, combined with (2) of Theorem 6.27, will ultimately allow us to construct $\mathcal{U}$-frequently hypercyclic operators of $\mathrm{C}_{+, 2}$-type which are not frequently hypercyclic.

Theorem 6.28. - Let $T=T_{v, w, \varphi, b}$ be an operator of $C_{+, 2}$-type on $\ell_{p}(\mathbb{N})$, and for every $k \geq 1$, set $\gamma_{k}:=2^{\delta^{(k-1)}-\tau^{(k)}}\left(\Delta^{(k)}\right)^{1-\frac{1}{p}}$. Suppose that the sequence $\left(\gamma_{k}\right)_{k \geq 1}$ is nonincreasing and that it satisfies the following three conditions:

$$
\sum_{k \geq 1} 2^{k} \gamma_{k}^{1 / 2} \leq 1, \quad \limsup _{k \rightarrow \infty} \frac{\tau^{(k)}}{\delta^{(k)}}<1, \quad \text { and } \quad \lim _{k \rightarrow \infty} \frac{\delta^{(k)}}{\delta^{(k+1)}}=0 .
$$

Then $T$ is frequently hypercyclic if and only if $\limsup _{k \rightarrow \infty} \delta^{(k)} / \Delta^{(k)}>0$.

Proof. - Since $\lim \sup _{k \rightarrow \infty} \frac{\tau^{(k)}}{\delta^{(k)}}<1$, the condition $\lim \sup _{k \rightarrow \infty} \frac{\delta^{(k)}}{\Delta^{(k)}}>0$ is equivalent to

$$
\limsup _{k \rightarrow \infty} \frac{\delta^{(k)}-\tau^{(k)}}{\Delta^{(k)}}>0
$$

and by assertion (3) of Theorem 6.27, this condition implies that $T$ is frequently hypercyclic.

The proof of the converse assertion proceeds exactly as in the proof of the implication $(1) \Longrightarrow(3)$ in Theorem 6.18, Suppose that $\lim \sup _{k \rightarrow \infty} \frac{\delta^{(k)}}{\Delta(k)}=0$, and let $x \in \ell_{p}(\mathbb{N})$ be a hypercyclic vector for $T$. Our aim is to prove that $x$ cannot be a frequently hypercyclic vector for $T$, using Theorem 6.10, We set

$$
\beta_{l}:=4 \gamma_{k} \quad \text { for every } l \in\left[2^{k-1}, 2^{k}\right), k \geq 1 .
$$


This sequence $\left(\beta_{l}\right)_{l \geq 1}$ is non-increasing and satisfies $\sum_{l \geq 1} \sqrt{\beta_{l}} \leq 1$. Setting

$$
X_{l}:=\left\|\sum_{i=b_{l}}^{b_{l+1}-1}\left(\prod_{s=i+1}^{b_{l+1}-1} w_{s}\right) x_{i} e_{i}\right\| \quad \text { for } \quad l \in\left[2^{k-1}, 2^{k}\right), k \geq 1,
$$

we have $\left\|P_{l} x\right\| \leq X_{l}$.

Also, by Fact 6.12, we have for every $l \geq 0$, every $0 \leq n<l$, and every $1 \leq N \leq \Delta^{(k)}$, where $l \in\left[2^{k-1}, 2^{k}\right)$, that

$$
\sup _{j \geq 0}\left\|P_{n} T^{j} P_{l} x\right\| \leq \frac{1}{4} \beta_{l} X_{l}
$$

and

$$
\sup _{0 \leq j \leq N}\left\|P_{n} T^{j} P_{l} x\right\| \leq \frac{1}{4} \beta_{l} \cdot\left(\prod_{i=\Delta^{(k)}-N+1}^{\Delta^{(k)}-1}\left|w_{i}^{(k)}\right|\right) \cdot\left\|P_{l} x\right\| .
$$

At this point, we diverge from the proof of Theorem 6.18, and set $N_{l}:=\delta^{(k)}$ for every $l \in\left[2^{k-1}, 2^{k}\right)$ and every $k \geq 1$. We then have $\sup _{0 \leq j \leq N_{l}}\left\|P_{n} T^{j} P_{l} x\right\| \leq \frac{1}{4} \beta_{l}\left\|P_{l} x\right\|$ for every $l \geq 0$ and every $0 \leq n<l$. In order to check that assumption (C') of Theorem 6.10 holds true, we use again Fact 6.13. We have $w_{i}^{(k)}=1$ for every $i \in\left(\delta^{(k)}, \Delta^{(k)}-3 \delta^{(k)}\right)$ and

$$
\prod_{i=\delta^{(k)}+1}^{\Delta^{(k)}-1} w_{i}^{(k)}=1
$$

It follows from Fact 6.13 that

$$
\frac{1}{J+1} \#\left\{0 \leq j \leq J ;\left\|P_{l} T^{j} T_{l} x\right\| \geq X_{l} / 2\right\} \geq 1-8 \delta^{(k)}\left(\frac{1}{J+1}+\frac{1}{\Delta^{(k)}}\right)
$$

for every $J \geq 0$, every $k \geq 1$, and every $l \in\left[2^{k-1}, 2^{k}\right)$. Now, we have $\min \left(\varphi^{-1}(l)\right)=2^{k}$ for every $l \in\left[2^{k-1}, 2^{k}\right)$, so that $N_{\min \left(\varphi^{-1}(l)\right)}=\delta^{(k+1)}$.

For every $k \geq 1$, we have

$$
\inf _{J \geq \delta^{(k+1)}} \frac{1}{J+1} \#\left\{0 \leq j \leq J ;\left\|P_{l} T^{j} T_{l} x\right\| \geq X_{l} / 2\right\} \geq 1-\frac{8 \delta^{(k)}}{\delta^{(k+1)}}-\frac{8 \delta^{(k)}}{\Delta^{(k)}} .
$$

Hence

$$
\begin{aligned}
\liminf _{l \rightarrow \infty} \inf _{J \geq \delta^{(k+1)}} \frac{1}{J+1} \#\{0 \leq j \leq J ; & \left.\left\|P_{l} T^{j} T_{l} x\right\| \geq X_{l} / 2\right\} \\
& \geq \liminf _{k \rightarrow \infty}\left(1-\frac{8 \delta^{(k)}}{\delta^{(k+1)}}-\frac{8 \delta^{(k)}}{\Delta(k)}\right)=1,
\end{aligned}
$$

since $\lim _{k \rightarrow \infty} \frac{\delta^{(k)}}{\delta^{(k+1)}}=0$ and $\lim \sup _{k \rightarrow \infty} \frac{\delta^{(k)}}{\Delta^{(k)}}=0$. Theorem 6.10 thus implies that $T$ is not frequently hypercyclic 
6.7.2. UFHC but not FHC. - As a direct consequence of Theorems 6.27 and 6.28, we now obtain

Theorem 6.29. - Let $T=T_{v, w, \varphi, b}$ be an operator of $C_{+, 2}$-type on $\ell_{p}(\mathbb{N})$. For every $k \geq$ 1 , set $\gamma_{k}:=2^{\delta^{(k-1)}-\tau^{(k)}}\left(\Delta^{(k)}\right)^{1-\frac{1}{p}}$. Suppose that the sequence $\left(\gamma_{k}\right)_{k \geq 1}$ is non-increasing, and that the following conditions are satisfied:

$$
\sum_{k \geq 1} 2^{k} \gamma_{k}^{1 / 2} \leq 1, \quad \limsup _{k \rightarrow \infty} \frac{\tau^{(k)}}{\delta^{(k)}}<1, \quad \lim _{k \rightarrow \infty} \frac{\delta^{(k)}}{\delta^{(k+1)}}=0 \quad \text { and } \quad \lim _{k \rightarrow \infty} \frac{\delta^{(k)}}{\Delta^{(k)}}=0 .
$$

Then $T$ is $\mathcal{U}$-frequently hypercyclic but not frequently hypercyclic.

Here is a concrete example, the proof of which is left to the reader.

Example 6.30. - If we consider the operator of $\mathrm{C}_{+, 2}$-type associated to the parameters

$$
\tau^{(k)}=2^{C k^{2}}, \quad \delta^{(k)}=2^{C k^{2}+1} \quad \text { and } \quad \Delta^{(k)}=2^{2 C k^{2}+4}, \quad k \geq 1,
$$

where $C$ is a sufficiently large integer, then $T$ is $\mathcal{U}$-frequently hypercyclic but not frequently hypercyclic.

6.8. Operators of $\mathrm{C}_{2}$-type: chaos plus mixing do not imply UFHC. - In this subsection, we introduce yet another class of C-type operators $T=T_{v, w, \varphi, b}$ on $\ell_{p}(\mathbb{N})$.

We consider increasing sequences $\left(a_{k}\right)_{k>1},\left(f_{k}\right)_{k \geq 1},\left(\delta^{(k)}\right)_{k \geq 1},\left(\tau^{(k)}\right)_{k \geq 1}$ and $\left(\Delta^{(k)}\right)_{k \geq 1}$ of integers such that $0 \leq a_{k} \leq f_{k}<\Delta^{(\bar{k})}-4 \delta^{(k)}$ for every $k \geq 1$, and we denote by $\left(J_{k}\right)_{k \geq 0}$ the partition of $\mathbb{N}$ into consecutive finite intervals defined as follows: $J_{0}=\{0\}$ and $\# J_{k}=\left(f_{k}-a_{k}\right)\left(\sum_{i=0}^{k-1} \# J_{i}\right)$ for every $k \geq 1$.

We then require that:

- $\varphi(n)=\left\lfloor\frac{n-\min J_{k}}{f_{k}-a_{k}}\right\rfloor$ for every $n \in J_{k}$;

- the blocks $\left[b_{n}, b_{n+1}\right), n \in J_{k}$, all have the same size $\Delta^{(k)}$;

- the sequence $v$ is given by

$$
v_{n}=2^{-\tau^{(k)}} \quad \text { for every } n \in J_{k} \text {; }
$$

- If $j$ belongs to the interval $\left[b_{n}, b_{n+1}\right)$ with $n \in J_{k}$ and $\left(n-\min J_{k}\right) \bmod \left(f_{k}-a_{k}\right)=l$, then the weight $w_{j}$ is given by

$$
w_{j}= \begin{cases}2 & \text { if } b_{n}<j \leq b_{n}+\delta^{(k)} \\ 1 & \text { if } b_{n}+\delta^{(k)}<j<b_{n+1}-a_{k}-l-2 \delta^{(k)} \\ 1 / 2 & \text { if } b_{n+1}-a_{k}-l-2 \delta^{(k)} \leq j<b_{n+1}-a_{k}-l-\delta^{(k)} \\ 2 & \text { if } b_{n+1}-a_{k}-l-\delta^{(k)} \leq j<b_{n+1}-a_{k}-l \\ 1 & \text { if } b_{n+1}-a_{k}-l \leq j<b_{n+1} .\end{cases}
$$

If these conditions are met, we call $T=T_{v, w, \varphi, b}$ an operator of $C_{2}$-type.

Note that the family of $\mathrm{C}_{2}$-type operators contain that of $\mathrm{C}_{+, 2}$-type operators. Indeed, if we consider the sequences $\left(a_{k}\right)_{k \geq 1}$ and $\left(f_{k}\right)_{k \geq 1}$ given by $a_{k}=\delta^{(k)}$ and $f_{k}=a_{k}+1$ for every $k \geq 1$, we get back the definition of $\mathrm{C}_{+, 2}$-type operators. The specificity of these new operators lies in the fact that for every $k \geq 1$ and every $n \in \bigcup_{k^{\prime}<k} J_{k^{\prime}}$, the set $J_{k}$ contains $f_{k}-a_{k}$ integers $m$ for which $\varphi(m)=n$, and, for each of these integers, the central block of weights $(1 / 2, \ldots, 1 / 2,2, \cdots, 2)$ is translated in a similar way. 
6.8.1. How to be topologically mixing. - By using Proposition 6.14, we can show that under suitable assumptions on the sequences $\left(a_{k}\right)_{k \geq 1}$ and $\left(f_{k}\right)_{k \geq 1}$, an operator of $\mathrm{C}_{2}$-type can be topologically mixing.

Proposition 6.31. - Let $T$ be an operator of $C_{2}$-type on $\ell_{p}(\mathbb{N})$. Suppose that the following two conditions hold true: $\lim _{k \rightarrow \infty}\left(\delta^{(k)}-\tau^{(k)}\right)=\infty$, and the set

$$
\bigcup_{k \geq 1}\left[a_{k}+\delta^{(k)}, f_{k}+\delta^{(k)}\right)
$$

is cofinite. Then $T$ is topologically mixing.

Proof. - With the notations of Proposition 6.14, we have to show that the set $S_{\eta, n}$ is cofinite. First of all, observe that the set $\mathcal{N}_{\eta, n}$ contains, for every $k \geq 1$ such that $2^{\delta^{(k)}-\tau^{(k)}}>1 / \eta$, the set $\varphi^{-1}(n) \cap J_{k}$. Since $\delta^{(k)}-\tau^{(k)}$ tends to infinity as $k$ tends to infinity, there exists an integer $k_{0} \geq 1$ such that $\mathcal{N}_{\eta, n}$ contains the set

$$
\bigcup_{k \geq k_{0}}\left(\varphi^{-1}(n) \cap J_{k}\right)
$$

Recalling that

$$
S_{\eta, n}=\bigcup_{m \in \mathcal{N}_{\eta, n}}\left\{s \in\left[1, b_{m+1}-b_{m}\right) ;\left|v_{m}\right| \prod_{i=b_{m+1}-s}^{b_{m+1}-1}\left|w_{i}\right|>\frac{1}{\eta}\right\},
$$

we deduce that $S_{\eta, n}$ contains the set

$$
\bigcup_{k \geq k_{0}} \bigcup_{m \in \varphi^{-1}(n) \cap J_{k}}\left\{s \in\left[1, b_{m+1}-b_{m}\right) ;\left|v_{m}\right| \prod_{i=b_{m+1}-s}^{b_{m+1}-1}\left|w_{i}\right| \geq 2^{\delta^{(k)}-\tau^{(k)}}\right\}
$$

which in its turn contains the set

$$
\bigcup_{k \geq k_{0}} \bigcup_{m \in \varphi^{-1}(n) \cap J_{k}}\left\{s \in\left[1, b_{m+1}-b_{m}\right) ; \prod_{i=b_{m+1}-s}^{b_{m+1}-1}\left|w_{i}\right|=2^{\delta^{(k)}}\right\}
$$

(recall that $v_{m}=2^{-\tau^{(k)}}$ for every integer $m$ belonging to $J_{k}$ ). Fix an integer $k \geq k_{0}$, and let $m$ be an integer of the form $m=\min J_{k}+n\left(f_{k}-a_{k}\right)+l$, where $0 \leq l<f_{k}-a_{k}$. Then $m$ belongs to $J_{k}, \varphi(m)=n$ and $\prod_{i=b_{m+1}-a_{k}-l-\delta^{(k)}}^{b_{m+1}-1}\left|w_{i}\right|=2^{\delta^{(k)}}$. It follows that $S_{\eta, n}$ contains every integer of the form $a_{k}+l+\delta^{(k)}$, where $0 \leq l<f_{k}-a_{k}$, i.e. that

$$
\bigcup_{k \geq k_{0}}\left[a_{k}+\delta^{(k)}, f_{k}+\delta^{(k)}\right) \subseteq S_{\eta, n}
$$

Since the set on the left hand side is cofinite by assumption, the desired result follows from Proposition 6.14. 
6.8.2. How not to be UFHC. - We now give some conditions ensuring that a $\mathrm{C}_{2}$-type operator fails to be $\mathcal{U}$-frequently hypercyclic. This will rely on the following more general version of Fact 6.13 ,

Fact 6.32. - Let $T$ be an operator of C-type on $\ell_{p}(\mathbb{N})$, and let $x \in \ell_{p}(\mathbb{N})$. Fix $l \geq 0$, and define

$$
X_{l}:=\left\|\sum_{k=b_{l}}^{b_{l+1}-1}\left(\prod_{s=k+1}^{b_{l+1}-1} w_{s}\right) x_{k} e_{k}\right\| .
$$

Suppose that there exist three integers $0 \leq k_{0}<k_{1}<k_{2} \leq b_{l+1}-b_{l}$ such that

$$
\left|w_{b_{l}+k}\right|=1 \quad \text { for every } k \in\left(k_{0}, k_{1}\right) \cup\left(k_{2}, b_{l+1}-b_{l}\right) \text { and } \prod_{s=b_{l}+k_{0}+1}^{b_{l+1}-1}\left|w_{s}\right|=1 \text {. }
$$

Then we have for every $J \geq 0$

$$
\begin{aligned}
\frac{1}{J+1} \#\{0 \leq j \leq & \left.j\left\|P_{l} T^{j} P_{l} x\right\| \geq X_{l} / 2\right\} \\
& \geq 1-4\left(k_{2}-k_{1}+k_{0}\right) \cdot\left(\frac{1}{J+1}+\frac{1}{b_{l+1}-b_{l}}\right) .
\end{aligned}
$$

Proof. - The proof is similar to the proof of Fact 6.13 except that the sets $I_{j}, j \geq 0$, have to be defined as follows:

$I_{j}:=\left\{k \in\left[b_{l}, b_{l+1}\right) ; j+k-b_{l} \bmod \left(b_{l+1}-b_{l}\right)\right.$ does not belong to $\left.\left[k_{0}, k_{1}\right) \cup\left[k_{2}, b_{l+1}-b_{l}\right)\right\}$.

We can then deduce that there exists an integer $j_{0}$ such that for every $J \geq 0$ :

$$
\begin{aligned}
\frac{1}{J+1} \#\left\{0 \leq j \leq J ;\left\|P_{l} T^{j} P_{l} x\right\|\right. & \left.\geq X_{l} / 2\right\} \\
& \geq 1-\frac{1}{J+1} \#\left\{0 \leq j \leq J ; I_{j} \cap I_{j_{0}} \neq \emptyset\right\} .
\end{aligned}
$$

Now, we remark that if we set $i_{j}:=j \bmod \left(b_{l+1}-b_{j}\right)$ for every $j \geq 0$, we have

$$
I_{j}=\left\{\begin{array}{l}
{\left[b_{l}, b_{l}+k_{0}-i_{j}\right) \cup\left[b_{l}+k_{1}-i_{j}, b_{l}+k_{2}-i_{j}\right) \cup\left[b_{l+1}-i_{j}, b_{l+1}\right) \quad \text { if } 0 \leq i_{j}<k_{0}} \\
{\left[b_{l}+k_{1}-i_{j}, b_{l}+k_{2}-i_{j}\right) \cup\left[b_{l+1}-i_{j}, b_{l+1}+k_{0}-i_{j}\right) \quad \text { if } k_{0} \leq i_{j} \leq k_{1}} \\
{\left[b_{l}, b_{l}+k_{2}-i_{j}\right) \cup\left[b_{l+1}-i_{j}, b_{l+1}+k_{0}-i_{j}\right) \cup\left[b_{l+1}+k_{1}-i_{j}, b_{l+1}\right) \quad \text { if } k_{1}<i_{j}<k_{2}} \\
{\left[b_{l+1}-i_{j}, b_{l+1}+k_{0}-i_{j}\right) \cup\left[b_{l+1}+k_{1}-i_{j}, b_{l+1}+k_{2}-i_{j}\right) \quad \text { if } k_{2}<i_{j}<b_{l+1}-b_{l} .}
\end{array}\right.
$$

This particular structure of the sets $I_{j}$ implies that

$$
\#\left\{0 \leq j \leq J ; I_{j} \cap I_{j_{0}} \neq \emptyset\right\} \leq 4\left(k_{2}-k_{1}+k_{0}\right) \cdot\left(\left\lfloor\frac{J}{b_{l+1}-b_{l}}\right\rfloor+1\right),
$$

and this yields the desired result.

Proposition 6.33. - Let $T$ be an operator of $C_{2}$-type on $\ell_{p}(\mathbb{N})$, and define as usual a sequence $\left(\gamma_{k}\right)_{k \geq 1}$ by setting $\gamma_{k}=2^{\delta^{(k-1)}-\tau^{(k)}}\left(\Delta^{(k)}\right)^{1-\frac{1}{p}}$ for every $k \geq 1$. Suppose that the sequence $\left(\gamma_{k}\right)_{k \geq 1}$ is non-increasing, and that the following two conditions are satisfied:

$$
2 \sum_{k \geq 1} \# J_{k} \gamma_{k}^{1 / 2} \leq 1 \quad \text { and } \quad \lim _{k \rightarrow \infty} \frac{\delta^{(k)}}{a_{k}}=0 .
$$

Then $T$ is not $\mathcal{U}$-frequently hypercyclic. 
Proof. - Let $x \in \ell_{p}(\mathbb{N})$ be a hypercyclic vector for $T$. Our aim is to prove that $x$ cannot be a $\mathcal{U}$-frequently hypercyclic vector for $T$, using Theorem 6.10, We set

$$
\beta_{l}:=4 \gamma_{k} \quad \text { for every } l \in J_{k} \text { and every } k \geq 1 \text {. }
$$

This sequence $\left(\beta_{l}\right)_{l \geq 1}$ is non-increasing and satisfies $\sum_{l \geq 1} \sqrt{\beta_{l}} \leq 1$. Setting

$$
X_{l}:=\left\|\sum_{k=b_{l}}^{b_{l+1}-1}\left(\prod_{j=k+1}^{b_{l+1}-1} w_{j}\right) x_{k} e_{k}\right\| \quad \text { for every } l \in I_{k} \text { and every } k \geq 1,
$$

we have $\left\|P_{l} x\right\| \leq X_{l}$ and, by Fact 6.12 ,

$$
\sup _{j \geq 0}\left\|P_{n} T^{j} P_{l} x\right\| \leq \frac{1}{4} \beta_{l} X_{l}
$$

for every $0 \leq n<l$, and

$$
\sup _{0 \leq j \leq N}\left\|P_{n} T^{j} P_{l} x\right\| \leq \frac{1}{4} \beta_{l}\left(\sup _{b_{l+1}-N \leq k<b_{l+1}} \prod_{s=k+1}^{b_{l+1}-1}\left|w_{s}\right|\right)\left\|P_{l} x\right\|
$$

for every $1 \leq N \leq \Delta^{(k)}$ and $0 \leq n<l$ with $l$ belonging to $J_{k}$. Setting $N_{l}:=a_{k}$ for every $l \in J_{k}$ and every $k \geq 1$, we deduce that

$$
\sup _{0 \leq j \leq N_{l}}\left\|P_{n} T^{j} P_{l} x\right\| \leq \frac{1}{4} \beta_{l}\left\|P_{l} x\right\|
$$

for every $l \geq 0$ and every $0 \leq n<l$. In order to check that assumption (C) of Theorem6.10 holds true, we use Fact 6.32. Let $n$ belong to $J_{k}$, and define $r=\left(n-\min J_{k}\right) \bmod \left(f_{k}-a_{k}\right)$. We have $w_{b_{n}+j}=1$ for every $j \in\left(\delta^{(k)}, \Delta^{(k)}-a_{k}-r-2 \delta^{(k)}\right) \cup\left(\Delta^{(k)}-a_{k}-r, \Delta^{(k)}\right)$ and

$$
\prod_{j=b_{n}+\delta^{(k)}+1}^{b_{n+1}-1} w_{j}=1
$$

It follows from Fact 6.32 that

$$
\frac{1}{J+1} \#\left\{0 \leq j \leq J ;\left\|P_{l} T^{j} T_{l} x\right\| \geq X_{l} / 2\right\} \geq 1-12 \delta^{(k)}\left(\frac{1}{J+1}+\frac{1}{\Delta^{(k)}}\right)
$$

for every $J \geq 0$, every $k \geq 1$, and every $l \in J_{k}$. For every $k \geq 1$, we thus have

$$
\inf _{J \geq a_{k}} \frac{1}{J+1} \#\left\{0 \leq j \leq J ;\left\|P_{l} T^{j} T_{l} x\right\| \geq X_{l} / 2\right\} \geq 1-\frac{12 \delta^{(k)}}{a_{k}}-\frac{12 \delta^{(k)}}{\Delta^{(k)}} .
$$

Since $\lim _{k \rightarrow \infty} \frac{\delta^{(k)}}{\Delta^{(k)}} \leq \lim _{k \rightarrow \infty} \frac{\delta^{(k)}}{a_{k}}=0$, it follows that

$$
\begin{aligned}
\liminf _{l \rightarrow \infty} \inf _{J \geq N_{l}} \frac{1}{J+1} \#\{0 \leq j \leq J ; & \left.\left\|P_{l} T^{j} T_{l} x\right\| \geq X_{l} / 2\right\} \\
& \geq \liminf _{k \rightarrow \infty}\left(1-\frac{12 \delta^{(k)}}{a_{k}}-\frac{12 \delta^{(k)}}{\Delta^{(k)}}\right)=1 .
\end{aligned}
$$

By Theorem 6.10, $T$ is not $\mathcal{U}$-frequently hypercyclic. 
6.8.3. Chaotic and mixing operators which are not UFHC. - From Proposition 6.31 and 6.33, we immediately deduce

Theorem 6.34. - Let $T$ be an operator of $C_{2}$-type on $\ell_{p}(\mathbb{N})$, and for every $k \geq 1$, set $\gamma_{k}=2^{\delta^{(k-1)}-\tau^{(k)}}\left(\Delta^{(k)}\right)^{1-\frac{1}{p}}$. Suppose that the sequence $\left(\gamma_{k}\right)_{k \geq 1}$ is non-increasing and that the following three conditions are met:

$$
2 \sum_{k \geq 1} \# J_{k} \gamma_{k}^{1 / 2} \leq 1, \quad \lim _{k \rightarrow \infty} \frac{\delta^{(k)}}{a_{k}}=0 \quad \text { and } \quad \lim _{k \rightarrow \infty}\left(\delta^{(k)}-\tau^{(k)}\right)=\infty .
$$

If the set

$$
\bigcup_{k \geq 1}\left[a_{k}+\delta^{(k)}, f_{k}+\delta^{(k)}\right)
$$

is cofinite, then $T$ is chaotic and topologically mixing but not $\mathcal{U}$-frequently hypercyclic.

Here is a concrete example of such a chaotic and topologically mixing operator which is not $\mathcal{U}$-frequently hypercyclic.

Example 6.35. - Let $C$ be a positive integer. Consider the operator of $\mathrm{C}_{2}$-type $T$ on $\ell_{p}(\mathbb{N})$ associated to the parameters

$\Delta^{(k)}=2^{2 C k^{2}+5}, \quad \delta^{(k)}=2^{C k^{2}+1}, \quad \tau^{(k)}=2^{C k^{2}}, \quad a_{k}=k \delta^{(k)} \quad$ and $\quad f_{k}=\frac{1}{2} \Delta^{(k)}, \quad k \geq 1$.

If $C$ is sufficiently large, then $T$ is chaotic and topologically mixing but not $\mathcal{U}$-frequently hypercyclic.

Proof. - The operator $T$ is well-defined since

$$
\Delta^{(k)}-4 \delta^{(k)}=2^{2 C k^{2}+5}-2^{C k^{2}+3}>2^{2 C k^{2}+4}=f_{k} \quad \text { and } \quad f_{k}=2^{2 C k^{2}+4} \geq k 2^{C k^{2}+1}=a_{k}
$$

for every $k \geq 1$. Moreover, the set

$$
\bigcup_{k \geq 1}\left[a_{k}+\delta^{(k)}, f_{k}+\delta^{(k)}\right)
$$

is cofinite since $f_{k}+\delta^{(k)} \geq a_{k+1}+\delta^{(k+1)}$ if $k$ is large enough. Indeed,

$$
f_{k}+\delta_{k}-a_{k+1}-\delta^{(k+1)} \geq f_{k}-2 a_{k+1}=2^{2 C k^{2}+4}-k 2^{C(k+1)^{2}+2},
$$

and the quantity on the right hand side tends to infinity as $k$ tends to infinity. Finally, since $\# J_{k} \leq \Delta^{(k)} k$. $\# J_{k-1}$ and $\# J_{0}=1$, we have $\# J_{k} \leq \prod_{j=1}^{k} j \Delta^{(j)} \leq 2^{8 C k^{3}}$ for every $k \geq 1$. It follows that the remaining assumptions of Theorem 6.34 are also satisfied if $C$ is sufficiently large.

Remark 6.36. - The operators constructed in 39] (which are chaotic and not $\mathcal{U}$-frequently hypercyclic) are never topologically mixing. Indeed, the parameters in this construction satisfy the following three conditions:

- the quantity $\delta_{n} /\left(b_{n+1}-b_{n}\right)$ tends to 0 as $n$ tends to infinity;

- for every $n \geq 1, b_{n+1}-b_{n}$ is a multiple of $2\left(b_{n}-b_{n-1}\right)$;

- for every $n \geq 1,2^{\delta_{n-1}-\tau_{n}}\left(b_{n+1}-b_{n}\right) \leq 2^{-2(n+1)}$. 
Therefore, for any such operator $T$, there exists an integer $n_{0}$ such that

$$
\delta_{n}<\frac{1}{2}\left(b_{n+1}-b_{n}\right) \quad \text { for every } n \geq n_{0} .
$$

It follows that for every integer $n \geq n_{0}$ such that 1 does not belong to the set $\bigcup_{j \geq 0} \varphi^{j}(n)$, $b_{n+1}-b_{n}$ does not belong to $\mathcal{N}_{T}\left(B(0,1), B\left(3 e_{b_{1}}, 1\right)\right)$. Indeed, we have for any $k \geq 0$

- $T^{b_{n+1}-b_{n}} e_{k}=e_{k}$ if $k<b_{n}$;

- $P_{1} T^{b_{n+1}-b_{n}} e_{k}=0$ if $k$ belongs to $\left[b_{n}, b_{n+1}\left[\right.\right.$ since 1 does not belong to $\bigcup_{j \geq 0} \varphi^{j}(n)$;

- $\left\|P_{1} T^{b_{n+1}-b_{n}} P_{l} x\right\| \leq 2^{-2(l+1)}\left\|P_{l} x\right\|$ if $l>n$, by Fact 6.12 .

Hence, if $x$ is any vector of the unit ball $B(0,1)$, the vector $y:=T^{b_{n+1}-b_{n}} x$ satisfies

$$
\left|y_{b_{1}}\right| \leq\left|x_{b_{1}}\right|+\sum_{l>n}\left\|P_{1} T^{b_{n+1}-b_{n}} P_{l} x\right\|<1+\sum_{l>n} \frac{1}{2^{2(l+1)}} \leq 2 .
$$

Thus $y$ does not belong to the ball $B\left(3 e_{b_{1}}, 1\right)$. Since there are infinitely many integers $n \geq$ $n_{0}$ such that $\bigcup_{j \geq 0} \varphi^{j}(n)$ does not contain 1 , this shows that $T$ is indeed not topologically mixing.

6.9. C-type operators with few eigenvalues. - In this subsection, we exhibit a class of C-type operators having only countably many unimodular eigenvalues. This provides further examples of hypercyclic operators on $\ell_{p}(\mathbb{N})$ with only countably many unimodular eigenvalues, and such that the associated unimodular eigenvectors span the space (as mentioned previously, the question of the existence of such operators was raised by Flytzanis in $[22]$ ).

The general idea of the forthcoming construction is the following: if $T=T_{v, w, \varphi, b}$ is an operator of C-type on $\ell_{p}(\mathbb{N})$ and if the sequence $v=\left(v_{n}\right)_{n \geq 1}$ decreases extremely fast, then the unimodular eigenvalues of $T$ must be roots of unity. This is not such a surprising statement if one considers what happens in the "degenerate" case where the sequence $v$ is identically equal to 0 : indeed, in this case the operator $T$ has the form $T=\bigoplus_{n \geq 0} C_{n}$ where the operators $C_{n}, n \geq 0$, are finite dimensional cyclic operators satisfying $C_{n}^{2\left(b_{n+1}-b_{n}\right)}=I$. Thus any eigenvalue $\lambda$ of $T$ must satisfy $\lambda^{2\left(b_{n+1}-b_{n}\right)}=1$ for some integer $n \geq 0$.

Before starting our construction, we determine the spectrum of the operators of C-type on $\ell_{p}(\mathbb{N})$ which we consider here:

Fact 6.37. - Let $T$ be a hypercyclic C-type operator on $\ell_{p}(\mathbb{N})$ such that

$$
\lim _{n \rightarrow \infty} \prod_{j=b_{n}+1}^{b_{n+1}-1}\left|w_{j}\right|=\infty
$$

Then the spectrum of $T$ is the closed disk $\bar{D}(0, R)$, where

$$
R:=\limsup _{N \rightarrow \infty}\left(\sup _{\substack{n \geq 0 \\ b_{n+1}-b_{n}>N}} \sup _{b_{n} \leq k<b_{n+1}-N}\left(w_{k+1} w_{k+2} \cdots w_{k+N}\right)\right)^{1 / N} .
$$

If $T$ is either a $C_{+, 1}$-type or a $C_{+, 2}$-type operator on $\ell_{p}(\mathbb{N})$, the spectrum of $T$ is thus the closed disk $\bar{D}(0,2)$. 
Proof. - Using the notation employed in the proof of Fact 6.2, we observe that $T$ is a compact perturbation of the direct sum operator $C:=\bigoplus_{n>0} C_{w, b, n}$ on $\ell_{p}(\mathbb{N})$. Also, the assumption of Fact 6.37 implies that $C$ is itself a compact perturbation of the forward weighted shift $S$ on $\ell_{p}(\mathbb{N})$ defined by

$$
S e_{k}= \begin{cases}w_{k+1} e_{k+1} & \text { if } k \in\left[b_{n}, b_{n+1}-1\right), n \geq 0 \\ 0 & \text { if } k=b_{n}-1, n \geq 1 .\end{cases}
$$

So $T^{*}$ is a compact perturbation of $S^{*}$, and it then follows from the Fredholm alternative that if $\lambda \in \mathbb{C}$ is any element of $\sigma\left(T^{*}\right) \backslash \sigma\left(S^{*}\right)$, then $\lambda$ is an eigenvalue of $T^{*}$. But as $T$ is hypercyclic, its adjoint has no eigenvalue, and it follows that $\sigma\left(T^{*}\right)$ is contained in $\sigma\left(S^{*}\right)$. Hence, $\sigma(T)$ is contained in $\sigma(S)$. Conversely, the same argument shows that any $\lambda \in \sigma(S) \backslash \sigma(T)$ is an eigenvalue of $S$. But the only eigenvalue of $S$ is 0 , so that $\sigma(S)$ is contained in $\sigma(T) \cup\{0\}$. Now, it is well-known that the $\sigma(S)$ is the closed disk $\bar{D}(0, R)$ (see for instance [46]), so that $\sigma(T) \subseteq \bar{D}(0, R) \subseteq \sigma(T) \cup\{0\}$. Since $\sigma(T)$ is closed, it follows that $\sigma(T)=\bar{D}(0, R)$, and this concludes the proof of Fact 6.37.

We now come back to our construction of C-type operators with few unimodular eigenvalues. In order to simplify the expressions involved in the results which we are about to state, we adopt the following notation: if $T=T_{v, w, \varphi, b}$ is an operator of C-type, we set

$$
\Delta b_{n}:=b_{n+1}-b_{n} \quad \text { for every } n \geq 0 .
$$

Also, we will say that an increasing sequence of positive integers $(n(m))_{m>0}$ is a $\varphi$-sequence if

$$
n(m)=\varphi(n(m+1)) \quad \text { for all } m \geq 0 .
$$

Theorem 6.38. - Let $T=T_{v, w, \varphi, b}$ be an operator of C-type on $\ell_{p}(\mathbb{N})$. Assume that for every $\varphi$-sequence $(n(m))_{m \geq 0}$, we have

$$
\limsup _{m \rightarrow \infty}\left|v_{n(m)}\right| \cdot 2^{n(m)}\left(\Delta b_{n(m)}\right)^{m}\left(\prod_{j=1}^{m-1} \frac{\left|v_{n(j)}\right|}{\Delta b_{n(j)}} \prod_{\nu=b_{n(j)}+1}^{b_{n(j)+1}-1}\left|w_{\nu}\right|\right)<\infty .
$$

Then each unimodular eigenvalue of $T$ is a root of unity. More precisely, any such eigenvalue $\lambda$ must satisfy $\lambda^{\Delta b_{n}}=1$ for some integer $n \geq 0$.

The condition appearing in Theorem 6.38 may look a bit strange since it involves all $\varphi$-sequences $(n(m))_{m \geq 0}$ (but it will show up naturally in the proof of Theorem 6.38). However, its general meaning is clear: $v_{n}$ should go to 0 quite fast as $n$ goes to infinity. Here is a consequence of Theorem 6.38 that makes it rather transparent. Recall that if $T$ is a $\mathrm{C}_{+, 1}$-type or a $\mathrm{C}_{+, 2}$-type operator on $\ell_{p}(\mathrm{~N})$, then $v_{n}=2^{-\tau^{(k)}}$ for every $n \in\left[2^{k-1}, 2^{k}\right)$ and every $k \geq 1$.

Corollary 6.39. - Let $T$ be a $C_{+, 1}$-type or a $C_{+, 2}$-type operator on $\ell_{p}(\mathbb{N})$. Assume that $\Delta^{(k)} / \Delta^{(k-1)}$ tends to infinity and $k \log \Delta^{(k)}=O\left(\Delta^{(k-1)}\right)$ as $k$ tends to infinity. If

$$
\lim _{k \rightarrow \infty} 2^{-\tau^{(k)}} M^{\Delta^{(k-1)}}=0 \quad \text { for every } M>0,
$$

then all the unimodular eigenvalues of $T$ must be roots of unity. 
Proof of Corollary 6.39, - Let $(n(m))_{m \geq 0}$ be a $\varphi$-sequence, and for each $m \geq 0$, let us denote by $\alpha_{m}$ the quantity appearing in (35), namely

$$
\alpha_{m}=v_{n(m)} \cdot 2^{n(m)}\left(\Delta b_{n(m)}\right)^{m}\left(\prod_{j=1}^{m-1} \frac{v_{n(j)}}{\Delta b_{n(j)}} \prod_{\nu=b_{n(j)}+1}^{b_{n(j)+1}-1} w_{\nu}\right) .
$$

For any $m \geq 0$, let us denote by $k_{m}$ the unique positive integer such that $n(m)$ belongs to the interval $\left[2^{k_{m}-1}, 2^{k_{m}}\right)$. Then $m \leq k_{m}$ because $(n(m))$ is a $\varphi$-sequence.

Observe first that the partial products

$$
\prod_{j=0}^{m-1} \frac{v_{n(j)}}{\Delta b_{n(j)}}, \quad m \geq 1
$$

remain bounded (in fact, they tend quickly to 0). Also, since the sequence $w$ is bounded, there exists a constant $A>1$ such that

$$
\prod_{j=1}^{m-1} \prod_{\nu=b_{n(j)}+1}^{b_{n(j)+1}-1}\left|w_{\nu}\right| \leq A^{\sum_{j=1}^{m-1} \Delta^{\left(k_{j}\right)}} \leq A \sum_{i=0}^{k_{m-1} \Delta^{(i)}} \quad \text { for every } m \geq 1 .
$$

Since $\Delta^{(k)} / \Delta^{(k-1)}$ tends to infinity as $k$ tends to infinity, it follows that there exists a positive constant $B$ such that

$$
\prod_{j=1}^{m-1} \prod_{\nu=b_{n(j)}+1}^{b_{n(j)+1}-1}\left|w_{\nu}\right| \leq B^{\Delta^{\left(k_{m}-1\right)}} \quad \text { for every } m \geq 1 .
$$

Moreover, there also exists a positive constant $C$ such that $2^{n(m)} \leq 2^{2^{k_{m}}} \leq C^{\Delta^{\left(k_{m}-1\right)}}$ for every $m \geq 1$ (again because $\Delta^{(k)} / \Delta^{(k-1)}$ tends to infinity). Lastly, we have

$$
\left(\Delta b_{n(m)}\right)^{m}=\left(\Delta^{\left(k_{m}\right)}\right)^{m} \leq\left(\Delta^{\left(k_{m}\right)}\right)^{k_{m}} \quad \text { for every } m \geq 1,
$$

and since $k \log \Delta^{(k)}=O\left(\Delta^{(k-1)}\right)$ the quantity $\left(\Delta^{\left(k_{m}\right)}\right)^{k_{m}}$ is dominated by $D^{\Delta^{\left(k_{m}-1\right)}}$ for some positive constant $D$. Putting things together, and remembering that $v_{n(m)}=2^{-\tau_{k_{m}}}$ for every $m \geq 1$, we obtain that there exists a positive constant $M$ such that

$$
\alpha_{m} \leq 2^{-\tau^{\left(k_{m}\right)}} M^{\Delta^{\left(k_{m}-1\right)}} \quad \text { for every } m \geq 1 .
$$

By (36), this concludes the proof of Corollary 6.39,

Remark 6.40. - Condition (36) is compatible with those appearing for example in Theorems 6.18, 6.22 or 6.29 ,

Proof of Theorem 6.38, - We start the proof with the following fact.

Fact 6.41. - Fix $\lambda \in \mathbb{T}$, and let $\left(p_{m}\right)_{m \geq 1}$ be a sequence of positive integers tending to infinity such that $p_{m+1}$ is a multiple of $p_{m}$ for every $m \geq 1$. Assume that $\lambda^{p_{m}} \neq \pm 1$ for every $m \geq 1$. Then there exist infinitely many integers $m \geq 1$ such that

$$
\left|\lambda^{p_{j}} \pm 1\right| \geq \frac{p_{j}}{p_{m+1}} \quad \text { for every } 1 \leq j \leq m
$$


Proof of Fact 6.41 - Writing $\lambda$ as $\lambda=e^{i \theta}$ with $\theta \in \mathbb{R} \backslash \pi \mathbb{Z}$, we have for every $j \geq 1$

$$
\left|\lambda^{p_{j}} \pm 1\right| \geq\left|\sin \left(p_{j} \theta\right)\right|=\sin \left(\operatorname{dist}\left(p_{j} \theta, \pi \mathbb{Z}\right)\right) \geq \frac{2}{\pi} \operatorname{dist}\left(p_{j} \theta, \pi \mathbb{Z}\right)=\frac{2 p_{j}}{\pi} \operatorname{dist}\left(\theta, \frac{\pi}{p_{j}} \mathbb{Z}\right) .
$$

Since $p_{m}$ is a multiple of $p_{j}$ for every $j \leq m$ and every $m \geq 1$, it follows that

$$
\left|\lambda^{p_{j}} \pm 1\right| \geq \frac{2 p_{j}}{\pi} \operatorname{dist}\left(\theta, \frac{\pi}{p_{m}} \mathbb{Z}\right) \quad \text { for every } m \geq 1 \text { and every } 1 \leq j \leq m .
$$

So it suffices to show that there exist infinitely many integers $m \geq 1$ such that

$$
\operatorname{dist}\left(\theta, \frac{\pi}{p_{m}} \mathbb{Z}\right) \geq \frac{\pi}{2 p_{m+1}} .
$$

Towards a contradiction assume that there exists an integer $m_{0}$ and, for every $m \geq m_{0}$, an integer $k_{m}$ such that

$$
\left|\theta-\frac{k_{m} \pi}{p_{m}}\right|<\frac{\pi}{2 p_{m+1}} \quad \text { for every } m \geq m_{0}
$$

We then have

$$
\left|\frac{k_{m} \pi}{p_{m}}-\frac{k_{m+1} \pi}{p_{m+1}}\right|<\frac{\pi}{2 p_{m+1}}+\frac{\pi}{2 p_{m+2}} \leq \frac{\pi}{p_{m+1}},
$$

and since $p_{m+1}$ is a multiple of $p_{m}$, it follows that

$$
\frac{k_{m} \pi}{p_{m}}=\frac{k_{m+1} \pi}{p_{m+1}} \quad \text { for every } m \geq m_{0}
$$

Since $p_{m}$ tends to infinity as $m$ tends to infinity, we conclude that $\theta=\frac{k_{m_{0}} \pi}{p_{m_{0}}}$, so that $\lambda^{p_{m_{0}}}= \pm 1$, which stands in contradiction with our initial assumption.

Let us now show that every unimodular eigenvalue $\lambda$ of $T$ satisfies $\lambda^{\Delta b_{n}}=1$ for some integer $n \geq 0$. Let $\lambda \in \mathbb{T}$ be a unimodular eigenvalue of $T$, and towards a contradiction, assume that $\lambda^{\Delta b_{n}} \neq 1$ for every $n \geq 0$. Note that since $\Delta b_{n+1}$ is an even multiple of $\Delta b_{n}$, we also have $\lambda^{\Delta b_{n}} \neq-1$ for every $n \geq 0$.

Let $x \in \ell_{p}(\mathbb{N}) \backslash\{0\}$ be an eigenvector of $T$ associated to the eigenvalue $\lambda$. The equation $T x=\lambda x$ yields that for every $n \geq 0$ and every $k \in\left[b_{n}, b_{n+1}\right)$, we have

$$
x_{k}=\frac{\prod_{\nu=b_{n}+1}^{k} w_{\nu}}{\lambda^{k-b_{n}}} x_{b_{n}}
$$

and

$$
\begin{aligned}
\lambda x_{b_{n}} & =-\frac{1}{\prod_{\nu=b_{n}+1}^{b_{n+1}-1} w_{\nu}} x_{b_{n+1}-1}+\sum_{l \in \varphi^{-1}(n)} v_{l} x_{b_{l+1}-1} \\
& =-\frac{1}{\lambda^{\Delta b_{n}-1}} x_{b_{n}}+\sum_{l \in \varphi^{-1}(n)} v_{l} \frac{\prod_{\nu=b_{l}+1}^{b_{l+1}-1} w_{\nu}}{\lambda^{\Delta b_{l}-1}} x_{b_{l}} .
\end{aligned}
$$

Since $\sum_{l>n} 2^{n-l}=1$, we deduce from the last identity that for every $n \geq 0$, there exists $l>n$ with $\varphi(l)=n$ such that

$$
\left|v_{l}\right| \prod_{\nu=b_{l}+1}^{b_{l+1}-1}\left|w_{\nu}\right|\left|x_{b_{l}}\right| \geq 2^{n-l}\left|\lambda+\frac{1}{\lambda^{\Delta b_{n}-1}}\right|\left|x_{b_{n}}\right|=2^{n-l}\left|\lambda^{\Delta b_{n}}+1\right|\left|x_{b_{n}}\right| .
$$

Thus, if we set

$$
\varepsilon_{n}=\left|1+\lambda^{\Delta b_{n}}\right|
$$


the following property holds true: for every $n \geq 0$, there exists $l>n$ with $\varphi(l)=n$ such that

$$
\left|x_{b_{l}}\right| \geq \frac{2^{n-l} \varepsilon_{n}}{\left|v_{l}\right| \prod_{\nu=b_{l}+1}^{b_{l+1}-1}\left|w_{\nu}\right|}\left|x_{b_{n}}\right| .
$$

Let us choose an integer $n(0)$ such that $x_{b_{n(0)}} \neq 0$ (since $x$ is non-zero, such an integer does exist by (37)). The argument given just above allows us to construct a $\varphi$-sequence $(n(m))_{m \geq 0}$ such that

$$
\left|x_{b_{n(m+1)}}\right| \geq \frac{2^{n(m)-n(m+1)} \varepsilon_{n(m)}}{\left|v_{n(m+1)}\right| \prod_{\nu=b_{n(m+1)}+1}^{b_{n(m+1)+1}-1}\left|w_{\nu}\right|}\left|x_{b_{n(m)}}\right| \quad \text { for every } m \geq 0 .
$$

We then have

$$
\left|x_{b_{n(m)}}\right| \geq \frac{2^{n(0)-n(m)}\left(\prod_{j=0}^{m-1} \varepsilon_{n(j)}\right)}{\left(\prod_{j=1}^{m}\left|v_{n(j)}\right|\right)\left(\prod_{j=1}^{m} \prod_{\nu=b_{n(j)}+1}^{b_{n(j)+1}-1}\left|w_{\nu}\right|\right)}\left|x_{b_{n(0)}}\right| \quad \text { for every } m \geq 1,
$$

and hence (by (37) again)

$$
\left|x_{b_{n(m)+1}-1}\right|=\frac{2^{n(0)-n(m)}\left(\prod_{j=0}^{m-1} \varepsilon_{n(j)}\right)}{\left(\prod_{j=1}^{m}\left|v_{n(j)}\right|\right)\left(\prod_{j=1}^{m-1} \prod_{\nu=b_{n(j)}+1}^{b_{n(j)+1}-1}\left|w_{\nu}\right|\right)}\left|x_{b_{n(0)}}\right| .
$$

If we now set

$$
\alpha_{m}:=\frac{\left(\prod_{j=1}^{m}\left|v_{n(j)}\right|\right)\left(\prod_{j=1}^{m-1} \prod_{\nu=b_{n(j)+1}}^{b_{n(j)+1}-1}\left|w_{\nu}\right|\right)}{2^{n(0)-n(m)}\left(\prod_{j=0}^{m-1} \varepsilon_{n(j)}\right)},
$$

it suffices to prove that $\alpha_{m}$ does not tend to infinity as $m$ tends to infinity in order to obtain a contradiction, since this would imply that $x$ does not belong to $c_{0}(\mathbb{N})$. In view of our assumption (35), this will be verified if we are able to show that

$$
\prod_{j=0}^{m-1} \varepsilon_{n(j)} \geq\left(\Delta b_{n(m)}\right)^{-m} \prod_{j=0}^{m-1} \Delta b_{n(j)} \quad \text { for infinitely many } m \geq 1 .
$$

At this point, we apply Fact 6.41, considering the sequence $\left(p_{m}\right)_{m \geq 1}$ defined by setting $p_{m}:=\Delta b_{n(m-1)}$ for every $m \geq 1$. The conclusion is that there exist infinitely many integers $m \geq 1$ such that

$$
\varepsilon_{j}=\left|1+\lambda^{\Delta b_{n(j)}}\right| \geq \frac{\Delta b_{n(j)}}{\Delta b_{n(m)}} \quad \text { for every } 0 \leq j \leq m-1,
$$

which gives (39). Theorem 6.38 is proved.

Remark 6.42. - Assume that $b_{1}=1$. If $\mathbf{n}=(n(m))_{m \geq 0}$ is a $\varphi$-sequence such that $n(0)=0$, the proof of Theorem 6.38 shows that if $\lambda \in \mathbb{T}$ and if we set

$$
x_{\mathbf{n}, \lambda}:=e_{0}+\sum_{m=1}^{\infty} \frac{\prod_{l=1}^{m}\left(1+\lambda^{\Delta b_{n(l-1)}}\right)}{\left(\prod_{l=1}^{m} v_{n(l)}\right)\left(\prod_{l=1}^{m-1} \prod_{\nu=b_{n(l)}+1}^{b_{n(l)+1}-1} w_{\nu}\right)} \sum_{j=b_{n(m)}}^{b_{n(m)+1}-1} \frac{\lambda^{b_{n(k)+1}-1-j}}{\prod_{\nu=j+1}^{b_{n(k)+1}-1} w_{\nu}} e_{j},
$$

then $x_{\mathbf{n}, \lambda}$ is an eigenvector of $T=T_{v, w, \varphi, b}$ associated to the eigenvalue $\lambda$, provided that the series defining $x_{\mathbf{n}, \lambda}$ is convergent. This has the following two interesting consequences. 
(1) An operator of C-type $T$ may quite well have a unimodular eigenvalue $\lambda$ such that the associated eigenspace $\operatorname{ker}(T-\lambda)$ is not one-dimensional. This happens in particular if $\lambda^{\Delta b_{n}}=-1$ for some integer $n$ and if there exists $m \neq n$ such that $\Delta b_{m}=\Delta b_{n}$.

(2) If we assume that $v_{n(l)}=\left(\prod_{\nu=b_{n(l-1)}+1}^{b_{n(l-1)+1}-1} w_{\nu}\right)^{-1}$, we obtain the following expression for $x_{\mathbf{n}, \lambda}$ :

$$
x_{\mathbf{n}, \lambda}=e_{0}+\sum_{m=1}^{\infty} \frac{\prod_{l=1}^{m}\left(1+\lambda^{\left.\Delta b_{n(l-1)}\right)}\right.}{v_{n(1)}} \sum_{j=b_{n(m)}}^{b_{n(m)+1}-1} \frac{\lambda^{b_{n(m)+1}-1-j}}{\prod_{\nu=j+1}^{b_{n(m)+1}-1} w_{\nu}} e_{j} .
$$

This vector is well-defined for some unimodular numbers $\lambda$ which are not roots of unity, provided that the sequence $\left(\Delta b_{n(l-1)}\right)_{l \geq 1}$ is suitably chosen. Therefore, operators of C-type can have unimodular eigenvalues which are not roots of unity.

6.10. $\mathbf{C}_{+, 1}$-type operators can be ergodic. - To conclude this section, we show that $\mathrm{C}_{+, 1}$-type operators can also have lots of eigenvalues, to the point of being even mixing in the Gaussian sense.

Theorem 6.43. - Let $T=T_{v, w, \varphi, b}$ be a $C_{+, 1}$-type operator on $\ell_{2}(\mathbb{N})$. Suppose that $b_{1}=1$, and that the series

$$
\sum_{m=1}^{\infty} 4^{m-\sum_{k=1}^{m}\left(\delta^{(k-1)}-\tau^{(k)}\right)} \Delta^{(m)}
$$

is convergent. Then $T$ is mixing in the Gaussian sense.

Proof. - Since $T$ is a $\mathrm{C}_{+}$-type operator, we know that the map $\varphi$ is given by $\varphi(n)=$ $n-2^{k-1}$ if $2^{k-1} \leq n<2^{k}, k \geq 1$. From this it follows that a sequence $\mathbf{n}=(n(m))_{m \geq 0}$ with $n(0)=0$ is a $\varphi$-sequence if and only if there exists an increasing sequence of positive integers $\left(k_{m}\right)_{m \geq 1}$ such that

$$
n(m)=2^{k_{1}-1}+\cdots+2^{k_{m}-1} \quad \text { for every } m \geq 1 .
$$

More precisely, $k_{m}$ is for each $m \geq 1$ the unique positive integer such that $2^{k_{m}-1} \leq n(m)<$ $2^{k_{m}}$. In this case, we say that $\mathbf{n}$ is the $\varphi$-sequence associated to the sequence $\left(k_{m}\right)_{m \geq 1}$.

With these notations, the formula of Remark 6.42 defining $x_{\mathbf{n}, \lambda}$ for a $\varphi$-sequence $\mathbf{n}$ and an element $\lambda$ of $\mathbb{T}$ can be rewritten as follows:

$$
\begin{aligned}
x_{\mathbf{n}, \lambda} & =e_{0}+\sum_{m=1}^{\infty} \frac{\prod_{l=1}^{m}\left(1+\lambda^{\Delta^{\left(k_{l-1}\right)}}\right)}{2^{\sum_{l=1}^{m} \tau^{\left(k_{l}\right)}+\sum_{l=1}^{m-1} \delta^{\left(k_{l}\right)}}} \sum_{j=b_{n(m)}}^{b_{n(m)+1}-1} \frac{\lambda^{b_{n(m)+1}-1-j}}{\max \left(1,2^{\delta^{\left(k_{m}\right)}-\left(j-b_{n(m)}\right)}\right)} e_{j} \\
& =e_{0}+\sum_{m=1}^{\infty} \frac{\prod_{l=1}^{m}\left(1+\lambda^{\Delta^{\left(k_{l-1}\right)}}\right)}{2^{\tau^{\left(k_{1}\right)}+\sum_{l=2}^{m}\left(\delta^{\left(k_{l-1}\right)}-\tau^{\left(k_{l}\right)}\right)}} \sum_{j=b_{n(m)}}^{b_{n(m)+1}-1} \frac{\lambda^{b_{n(m)+1}-1-j}}{\max \left(1,2^{\delta^{\left(k_{m}\right)}-\left(j-b_{n(m)}\right)}\right)} e_{j} .
\end{aligned}
$$

We will say that a $\varphi$-sequence $\mathbf{n}$ with $n(0)=0$ is a $\operatorname{good} \varphi$-sequence if $x_{\mathbf{n}, \lambda}$ is well-defined for every $\lambda \in \mathbb{T}$ and if the map $\lambda \mapsto x_{\mathbf{n}, \lambda}$ is continuous from $\mathbb{T}$ into $\ell_{2}(\mathbb{N})$.

Fact 6.44. - For any integer $n \geq 1$, there exists a good $\varphi$-sequence $\mathbf{n}$ passing through $n$, i.e. such that $n(r)=n$ for some integer $r \geq 1$. 
Proof of Fact 6.44 - Write $n$ as $n=2^{k_{1}-1}+\cdots+2^{k_{r}-1}$ with $1 \leq k_{1}<\cdots<k_{r}$, and set $k_{r+i}:=k_{r}+i$ for every $i \geq 1$. Then the $\varphi$-sequence $\mathbf{n}$ associated to $\left(k_{m}\right)_{m \geq 1}$ passes through $n$. Moreover, since $k_{r+i}:=k_{r}+i$ for every $i \geq 1$, it follows from the assumption that the series (40) is convergent that the series

$$
\sum_{m \geq 1} 4^{m-\sum_{l=1}^{m}\left(\delta^{\left(k_{l-1}\right)}-\tau^{\left(k_{l}\right)}\right)} \Delta^{\left(k_{m}\right)}
$$

is convergent as well. Since $\left|\prod_{l=1}^{m}\left(1+\lambda^{\Delta^{\left(k_{l}\right)}}\right)\right| \leq 2^{m}$ for every $m \geq 1$ and every $\lambda \in \mathbb{T}$, this implies that $\mathbf{n}$ is a good $\varphi$-sequence.

Fact 6.45. - Let $\mathbf{n}$ be a good $\varphi$-sequence. If $y=\sum_{j \geq 0} y_{j} e_{j}$ is a vector of $\ell_{2}(\mathbb{N})$ which is orthogonal to $x_{\mathbf{n}, \lambda}$ for every $\lambda \in \mathbb{T}$, then

$$
y_{0}=0 \quad \text { and } \quad y_{j}=0 \quad \text { for every } j \in \bigcup_{m \geq 1}\left[b_{n(m)}, b_{n(m)+1}\right) .
$$

Proof of Fact 6.45 - Let $\left(k_{m}\right)_{m \geq 1}$ be the increasing sequence of integers associated to $\mathbf{n}$, and set $k_{0}:=0$. By assumption and in view of the definition of $x_{\mathbf{n}, \lambda}$, we have

$$
y_{0}+\sum_{m=1}^{\infty} \prod_{l=1}^{m}\left(1+\lambda^{\Delta^{\left(k_{l-1}\right)}}\right) \sum_{b_{n(m)} \leq j<b_{n(m)+1}} c_{m, j} \lambda^{b_{n(m)+1}-1-j} y_{j}=0
$$

for every $\lambda \in \mathbb{T}$, where the $c_{m, j}$ are non-zero scalars which do not depend on $\lambda$.

Equality (41) applied to $\lambda:=-1$ yields that $y_{0}=0$. Indeed, since $\Delta^{\left(k_{0}\right)}=b_{1}-b_{0}=$ 1 , we have $\left(1+\lambda^{\Delta^{\left(k_{0}\right)}}\right)=0$, and the factor $\left(1+\lambda^{\Delta^{\left(k_{0}\right)}}\right)$ appears in each of the terms $\prod_{l=1}^{m}\left(1+\lambda^{\Delta^{\left(k_{l-1}\right)}}\right)$. Now, take $\lambda$ such that $\lambda^{\Delta^{\left(k_{1}\right)}}=-1$. Then $\prod_{l=1}^{m}\left(1+\lambda^{\Delta^{\left(k_{l-1}\right)}}\right)=0$ for every $m \geq 2$. Moreover, $\left(1+\lambda^{\Delta^{\left(k_{0}\right)}}\right)$ is non-zero because $\Delta^{\left(k_{1}\right)}$ is an even multiple of $\Delta^{\left(k_{0}\right)}$. So we get

$$
\sum_{b_{n(1)} \leq j<b_{n(1)+1}} c_{1, j} \lambda^{b_{n(1)+1}-1-j} y_{j}=0
$$

for every $\lambda$ such that $\lambda^{\Delta^{\left(k_{1}\right)}}=-1$. Denoting by $\Lambda_{1}$ the set of all such elements $\lambda$, and recalling that $n(1)=2^{k_{1}-1}$, we can rewrite (42) as

$$
\sum_{s=0}^{\Delta^{\left(k_{1}\right)}-1} z_{s} \lambda^{s}=0 \quad \text { for every } \lambda \in \Lambda_{1}
$$

where $z_{s}=c_{1, b_{n(1)+1}-1-s} y_{b_{n(1)+1}-1-s}$ for every $0 \leq s<\Delta^{\left(k_{1}\right)}$. Since $\Lambda_{1}$ has cardinality $\Delta^{\left(k_{1}\right)}$, it follows that $z_{s}=0$ for every $0 \leq s<\Delta^{\left(k_{1}\right)}$, and hence that $y_{j}=0$ for every $b_{n(1)} \leq j<b_{n(1)+1}$.

Continuing in this way, we obtain that for every $m \geq 1, y_{j}=0$ for every $j$ belonging to the interval $\left[b_{n(m)}, b_{n(m)+1}\right)$, which proves our claim.

It is now easy to conclude the proof of Theorem 6.43, Let us denote by $\mathbf{G}$ the set of all $\operatorname{good} \varphi$-sequences, and set $E_{\mathbf{n}}(\lambda):=x_{\mathbf{n}, \lambda}$ for every $\mathbf{n} \in \mathbf{G}$ and every $\lambda \in \mathbb{T}$. Then the maps $E_{\mathbf{n}}: \mathbb{T} \rightarrow \ell_{2}(\mathbb{N}), \mathbf{n} \in \mathbf{G}$, are continuous eigenvector fields for $T$ defined on $\mathbb{T}$. Facts 6.44 and 6.45 then imply that $\operatorname{span}\left\{E_{\mathbf{n}}(\lambda) ; \mathbf{n} \in \mathbf{G}, \lambda \in \mathbb{T}\right\}=\ell_{2}(\mathbb{N})$. By [8], it follows that $T$ is strongly mixing in the Gaussian sense. 
Example 6.46. - If we consider the $\mathrm{C}_{+, 1}$-type operator $T$ on $\ell_{2}(\mathbb{N})$ associated to the data $\delta^{(k)}=2 k, \tau^{(k)}=\frac{1}{2} \delta^{(k)}$ and $\Delta^{(k)}=2^{k+1}, k \geq 1$, then $T$ is mixing in the Gaussian sense.

Remark 6.47. - Condition (40) is incompatible with the "additional assumptions" of Theorem 6.18. Indeed, the latter imply that $\gamma_{k}$ tends to zero as $k$ tends to infinity, and hence that $\tau^{(k)}-\delta^{(k-1)}$ tends to infinity.

\section{A few questions}

We conclude the paper with a short list of questions. Most of them have already been stated in the paper, at least implicitly. Some of them will perhaps be found interesting and not hopelessly intractable.

Question 7.1. - For which Banach spaces $X$ with separable dual do the "typicality" results proved here for Hilbert spaces hold true?

Question 7.2. - Do there exist ergodic operators on $\mathcal{H}$ without eigenvalues?

Question 7.3. - Do there exist at least Hilbert space operators admitting non-trivial invariant measures but no eigenvalues?

Question 7.4. - Let $M>1$. What is the descriptive complexity of $\operatorname{ERG}_{M}(\mathcal{H})$ and $\operatorname{INV}_{M}(\mathcal{H})$, with respect to SOT and/or SOT*? In particular, are these sets Borel in $\mathfrak{B}_{M}(\mathcal{H})$ ?

Question 7.5. - Let $M>1$. Is $\operatorname{UFHC}_{M}(\mathcal{H})$ a true $\Pi_{4}^{0}$ set in $\left(\mathfrak{B}_{M}(\mathcal{H})\right.$, $\left.\mathrm{SOT}^{*}\right)$ ? Is $\mathrm{UFHC}_{M}(\mathcal{H}) \cap \mathrm{CH}_{M}(\mathcal{H})$ a true difference of $\Sigma_{3}^{0}$ sets?

Question 7.6. - Let $M>1$. Is $\operatorname{FHC}_{M}(\mathcal{H})$ Borel in $\mathfrak{B}_{M}(\mathcal{H})$ ?

Question 7.7. - Let $T \in \mathfrak{B}(\mathcal{H})$. Assume that $T$ is hypercyclic, and that there exists a sequence $\left(u_{i}\right)_{i \geq 1}$ of unimodular eigenvectors with rationally independent eigenvalues spanning a dense linear subspace of $\mathcal{H}$. Is $T$ ergodic, or at least frequently hypercyclic? $\mathcal{U}$-frequently hypercyclic?

Question 7.8. - Let $\boldsymbol{\lambda}$ be a sequence of distinct unimodular complex numbers tending to 1 , and let $\boldsymbol{\omega}$ be a bounded sequence of positive numbers. If the operator $T_{\boldsymbol{\lambda}, \boldsymbol{\omega}}=D_{\boldsymbol{\lambda}}+B_{\boldsymbol{\omega}}$ acting on $\ell_{2}(\mathbb{N})$ is hypercyclic, is it necessarily ergodic, or at least frequently hypercyclic? $\mathcal{U}$-frequently hypercyclic?

Question 7.9. - When exactly is an operator of the form $T_{\boldsymbol{\lambda}, \boldsymbol{\omega}}=D_{\boldsymbol{\lambda}}+B_{\boldsymbol{\omega}}$ hypercyclic?

Question 7.10. - Let $M>1$. Is the class of chaotic operators belonging to $\mathfrak{T}_{M}(\mathcal{H})$ comeager in $\mathfrak{T}_{M}(\mathcal{H})$ ? 
Question 7.11. - Let $T$ be a Banach space operator. Assume that $T$ has a dense set of uniformly recurrent points. Does it follow that $T$ has a non-zero periodic point?

Question 7.12. - Let $T$ be a hypercyclic operator. Assume that $c(T)>0$ and that $T$ admits an invariant measure with full support. Does it follow that $T$ is $\mathcal{U}$-frequently hypercyclic?

Question 7.13. - Let $X$ be a Banach space, and let $T \in \mathfrak{B}(X)$ have the OSP. Are the ergodic measures for $T$ dense in the space of all $T$-invariant measures?

Question 7.14. - On which Banach spaces is it possible to construct frequently hypercyclic operators which are not ergodic and/or $\mathcal{U}$-frequently hypercyclic operators which are not frequently hypercyclic?

\section{References}

[1] S. Bartoll, F. Martínez-Giménez, A. Peris, The specification property for backward shifts. J. Difference Equ. Appl. 18 (2012), 599-605.

[2] S. Bartoll, F. Martínez-Giménez, A. Peris, Operators with the specification property. J. Math. Anal. Appl. 436 (2016), 478-488.

[3] F. Bayart and T. Bermúdez, Semigroups of chaotic operators. Bull. Lond. Math. Soc. 41 (2009), 823-830.

[4] F. Bayart and S. Grivaux, Frequently hypercyclic operators. Trans. Amer. Math. Soc. 358 (2006), 5083-5117.

[5] F. Bayart and S. Grivaux, Invariant Gaussian measures for operators on Banach spaces and linear dynamics. Proc. Lond. Math. Soc. 94 (2007), 181-210.

[6] F. Bayart and É. Matheron, Hypercyclic operators failing the Hypercyclicity Criterion on classical Banach spaces. J. Funct. Anal 250 (2007), 426-441.

[7] F. Bayart and É. Matheron, Dynamics of linear operators. Cambridge Tracts in Mathematics 179, Cambridge University Press (2009).

[8] F. Bayart and É. Matheron, Mixing operators and small subsets of the circle. J. Reine Angew. Math. 715 (2016), 75-123.

[9] F. Bayart and I. Z. Rusza, Difference sets and frequently hypercyclic weighted shifts. Erg. Th. Dynam. Systems 35 (2015), 691-709.

[10] N. Bernardes, A. Bonilla, V. Müller and A. Peris, Distributional chaos for linear operators. J. Funct. Anal. 265 (2013), 2143-2163.

[11] J. Bès and A. Peris, Hereditarily hypercyclic operators. J. Funct. Anal. 167 (1999), 94-112.

[12] J. Bès, Q. Menet, A. Peris and Y. Puig, Recurrence properties of hypercyclic operators. Math. Ann. 366 (2016), 545-572.

[13] A. Bonilla and K.-G. Grosse-Erdmann, Frequently hypercyclic operators and vectors. Ergodic Theory Dynam. Systems 27 (2007), 383-404.

[14] A. Bonilla, K.-G. Grosse-Erdmann, Upper frequent hypercyclicity and related notions. Preprint (2016), available at http://arxiv.org/abs/1601.07276

[15] R. Bowen, Topological entropy and axiom A. In Global Analysis (Proc. Sympos. Pure Math., Vol. XIV, Berkeley, Calif., 1968), Amer. Math. Soc. (1970) 23-41.

[16] K. Chan, The density of hypercyclic operators on a Hilbert space. J. Operator Theory 47 (2002), 131-143. 
[17] I. Dayan, E. Glasner, Hypercyclic operators, Gauss measures and Polish dynamical systems. Israel J. Math. 208 (2015), 79 - 99.

[18] M. De La Rosa and C. Read, A hypercyclic operator whose direct sum is not hypercyclic. J. Operator Theory 61 (2009), 369-380.

[19] T. Eisner, A "typical" contraction is unitary. Enseign. Math. 56 (2010), 403-410.

[20] T. Eisner and A. Serény, Category theorems for stable operators on Hilbert spaces. Acta Sci. Math. (Szeged) 74 (2008), 259-270.

[21] T. Eisner, T. Mátrai, On typical properties of Hilbert space operators. Israel J. Math. 195 (2013), 247-281.

[22] E. Flytzanis, Unimodular eigenvalues and linear chaos in Hilbert spaces. Geom. Funct. Anal. 5 (1995), 1-13.

[23] H. Furstenberg, Recurrence in ergodic theory and combinatorial number theory. Princeton University Press (1981).

[24] E. Glasner, Ergodic theory via joinings. Mathematical Surveys and Monographs 101, Amer. Math. Soc. (2003).

[25] E. Glasner, B. Weiss, A universal hypercyclic representation. J. Funct. Anal. 268 (2015), 3478-3491.

[26] S. Grivaux, Construction of operators with prescribed behaviour. Arch. Math. 81 (2003), 291-299.

[27] S. Grivaux, A new class of frequently hypercyclic operators. Indiana Univ. Math. J. 60 (2011), 1177-1201.

[28] S. Grivaux, Some new examples of universal hypercyclic operators in the sense of Glasner and Weiss. To appear in Trans. Amer. Math. Soc.

[29] S. Grivaux, É. Matheron, Invariant measures for frequently hypercyclic operators. Adv. Math. 265 (2014), 371-427.

[30] S. Grivaux and S. Shkarin, Non-mixing hypercyclic operators. Unpublished work (2007).

[31] K. -G. Grosse-Erdmann and A. Peris, Linear Chaos. Universitext, Springer (2011).

[32] K. -G. Grosse-Erdmann and A. Peris, Frequently dense orbits. C. R. Math. Acad. Sci. 341 (2005), 123-128.

[33] P. R. Halmos, Lectures on ergodic theory. Chelsea Publishing Co. (1956).

[34] D. Herrero, Limits of hypercyclic and supercyclic operators. J. Funct. Anal. 99 (1991), 179190.

[35] D. Herrero, Hypercyclic operators and chaos. J. Operator Th. 29 (1992), 93-103.

[36] G. Kalisch, On operators on separable Banach spaces with arbitrary prescribed point spectrum. Proc. Amer. Math. Soc 34 (1972), 207-208.

[37] A. Kechris, Classical descriptive set theory. Graduate Texts in Mathematics, 156, Springer (1995).

[38] A. S. Kechris and A. Louveau, Descriptive set theory and the structure of sets of uniqueness. London Math. Soc. Lecture Notes Series 128, Cambridge University Press (1987).

[39] Q. Menet, Linear chaos and frequent hypercyclicity. To appear in Trans. Amer. Math. Soc.

[40] T. K. S. Moothathu, Two remarks on frequent hypercyclicity. J. Math. Anal. Appl. 408 (2013), 843-845.

[41] M. Murillo-Arcilla and A. Peris, Strong mixing measures for linear operators and frequent hypercyclicity. J. Math. Anal. Appl. 398 (2013), 462-465.

[42] K. R. Parthasarathy, On the category of ergodic measures. Illinois J. Math. 5 (1961), 648-656.

[43] G. Pedersen, Analysis now. Springer (1989).

[44] H. Salas, A hypercyclic operator whose adjoint is also hypercyclic. Proc. Amer. Math. Soc. 112 (1991), 765-770.

[45] O. Sarig, Lecture notes on ergodic theory. Lecture notes (2009), available online. 
[46] A. Shields, Weighted shift operators and analytic function theory. Topics in operator theory, Math. Surveys 13, 49 - 128, Amer. Math. Soc (1974).

[47] S. Shkarin, Universal elements for non-linear operators and their applications. J. Math. Anal. Appl. 348 (2008), 193-210.

[48] S. Shkarin, On the spectrum of frequently hypercyclic operators. Proc. Amer. Math. Soc. 137 (2009), 123-134.

[49] B. Schweizer and J. Smítal, Measure of chaos and a spectral decomposition of dynamical systems on the interval. Trans. Amer. Math. Soc. 344 (1994), 737-754.

[50] K. Sigmund, On dynamical systems with the Specification Property. Trans. Amer. Math. Soc. 190 (1974), 285-299.

[51] S. Todorcevic, Topics in topology. Lecture Notes in Mathematics 1652, Springer (1997).

[52] R. E. Waterman, Invariant subspaces, similarity and isometric equivalence of certain commuting operators on $L_{p}$. Pacific J. Math. 48 (1973), 593-613.

[53] N. Weaver, Set theory and cyclic vectors. J. Operator Theory 52 (2004), 133-138.

March 7, 2017

S. Grivaux, CNRS, Laboratoire Amiénois de Mathématique Fondamentale et Appliquée, UMR 7352, Université de Picardie Jules Verne, 33, rue Saint Leu, 80039 Amiens Cedex 1, France E-mail : sophie.grivaux@u-picardie.fr

É. Matheron, Laboratoire de Mathématiques de Lens, Université d'Artois, Rue Jean Souvraz SP 18, 62307 Lens, France • E-mail : etienne.matheron@univ-artois.fr

Q. Menet, Laboratoire de Mathématiques de Lens, Université d'Artois, Rue Jean Souvraz SP 18, 62307 Lens, France • E-mail : quentin.menet@univ-artois.fr 